\title{
A microscopy-based approach to understand polarized exocytosis during plant defence
}

Elysa Overdijk 


\section{Propositions}

1. The exocyst complex plays a key role at the plant-microbe interface. (this thesis)

2. The moss Physcomitrella patens is a valuable model system to study preinvasive plant defence at a cellular level.

(this thesis)

3. Cells translate perception of their environment into polarity.

4. Model systems define the framework of life sciences research.

5. Evidence-based teaching methods should be the standard in every academic classroom.

6. Raising kids is like performing a scientific experiment without the proper controls.

7. Making music together is a foundation for lifelong friendships.

Propositions belonging to the thesis, entitled

A microscopy-based approach

to understand polarized exocytosis during plant defence

Elysa Overdijk

Wageningen, 18 Oktober 2019. 


\section{A microscopy-based approach to understand polarized exocytosis during plant defence}

Elysa Overdijk 


\section{Thesis Committee}

\section{Promotor}

Prof. Dr Francine Govers

Personal chair at the Laboratory of Phytopathology

Wageningen University \& Research

\section{Co-promotors}

Dr Tijs Ketelaar

Assistant professor, Laboratory of Cell Biology

Wageningen University \& Research

Dr Klaas Bouwmeester

Research fellow, Laboratory of Phytopathology

Wageningen University \& Research

\section{Other members}

Prof. Dr Christa S. Testerink, Wageningen University \& Research

Dr Petra C. Boevink, The James Hutton Institute, Dundee, Scotland UK

Dr Michael J. Deeks, University of Exeter, UK

Dr Aska Goverse, Wageningen University \& Research

This research was conducted under the auspices of the Graduate School EPS (Experimental Plant Sciences). 


\section{A microscopy-based approach to understand polarized exocytosis during plant defence}

\section{Elysa-Maria Janna Regina Overdijk}

\section{Thesis}

Submitted in fulfillment of the requirements for the degree of doctor at Wageningen University by the authority of the Rector Magnificus,

Prof. Dr A.P.J. Mol, in the presence of the Thesis Committee appointed by the Academic Board to be defended in public on Friday 18 October 2019 at 11 a.m. in the Aula. 
Elysa Overdijk

A microscopy-based approach to understand polarized exocytosis during plant defence, 162 pages

PhD thesis, Wageningen University, Wageningen, The Netherlands (2019)

With references, with summaries in English and Dutch

ISBN: 978-94-6395-056-5

DOI: $10.18174 / 496953$ 
To Bram, my beloved husband 



\section{Abstract}

Plant diseases caused by pathogens threaten crop production worldwide. One of the approaches to combat pathogens is to improve the plant's own immunity, a strategy that requires detailed insight into the mechanisms underlying immunity. When plant cells are attacked by pathogens, their immune system is rapidly activated. One of the activated defence responses is a local and precise secretion of antimicrobial compounds and cell wall material at the site of attempted penetration. This requires polarized exocytosis. The central goal of this thesis was to understand polarized exocytosis in plant cells that are locally triggered by attack of a filamentous plant pathogen. We focused on the role of the exocyst complex during this process. The exocyst complex is a conserved multiprotein complex that facilitates one of the final steps of exocytosis. The research described in this thesis demonstrates the power of a multidisciplinary approach for unravelling plant-pathogen interactions. The data obtained by combining phytopathological and cell biological approaches show that the exocyst complex plays an important role in plant immunity against different unrelated pathogens and that exocyst subunits are recruited to sites of pathogen infection where they likely facilitate defence-related exocytosis to halt pathogen penetration. 



\section{Table of contents}

$\begin{array}{ll}\text { Abstract } & 7\end{array}$

$\begin{array}{lll}\text { Chapter } 1 & \text { General introduction } & 11\end{array}$

Chapter 2 Solanaceous exocyst subunits are involved in immunity to diverse plant pathogens

Chapter 3 Interaction between the moss Physcomitrella patens and

Phytophthora: a novel pathosystem for live-cell imaging of subcellular defence

Chapter 4 Time-gated confocal microscopy reveals accumulation of plant exocyst subunits at the plant-pathogen interface

Chapter 5 Phytophthora infestans RXLR effector AVR1 disturbs the growth of 83 Physcomitrella patens, without affecting Sec5 localization

References

Summary

Samenvatting

Acknowledgements

About the author 156

List of publications 157

Education statement 158 



\section{Chapter 1}

General introduction 


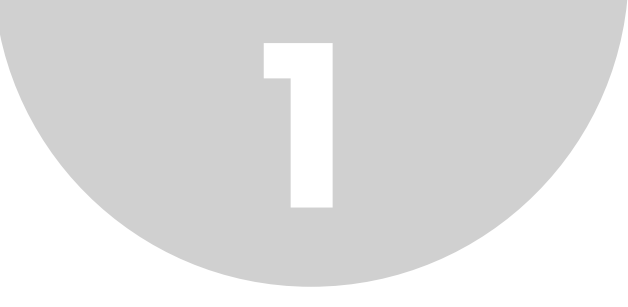

\section{General introduction}

Cell polarity, defined as the asymmetric distribution of any molecular or morphological feature within a cell, is a fundamental mechanism for cellular functioning in all organisms (Etienne-Manneville \& Hall, 2002). In plants, cell polarity is closely linked to development, growth and responses to the environment (Yang, 2008; Heider \& Munson, 2012; Lamour et al., 2012; Heider et al., 2016). It is for instance essential for the orientation of cell division during embryo development and lateral root formation, and for polar growth of pollen tubes and root hairs (Qi \& Greb, 2017). Conceptually, cell polarity may depend on either intrinsic signalling inside the cell, e.g. the asymmetric distribution of hormones, proteins or organelles, or on extrinsic signals present in the extracellular environment. The central goal of this thesis is to understand polarized exocytosis in plant cells that are locally triggered by attack of a filamentous plant pathogen. In this introductory chapter, we will first describe what is known in plants about the effects of pathogen infection on cell polarity and membrane trafficking. We will then address the role of the exocyst complex in plant immunity and the role of pathogen effectors in supressing plant immunity, in particular effectors that are known to manipulate membrane trafficking. We will conclude by outlining the research objectives of this thesis.

\section{Cell polarity and membrane trafficking during plant defence}

Plants are continuously confronted with a wide variety of microbial pathogens and pests, e.g. bacteria, viruses, nematodes, insects, fungi and oomycetes. They differ in their lifestyle and mode of infection. Many of the filamentous fungal and oomycete pathogens are biotrophs. Their hyphae penetrate plant cells and are often surrounded by a specialized plant-derived membrane (Inada \& Ueda, 2014). Upon contact and recognition of a pathogen, plant immunity (Box I) is rapidly activated and many cellular components are targeted to the infection site; the cytoplasm aggregated and organelles like the nucleus, chloroplasts, endoplasmic reticulum (ER), peroxisomes and Golgi bodies are transported towards the site of pathogen attack (Schmelzer, 2002; Takemoto et al., 2003). This targeted cytoplasmic streaming is achieved by reorganization of actin filaments (Hardham et al., 2008; Schmidt et al., 2014; Mayers et al., 2017). Furthermore, endocytotic and exocytotic membrane trafficking pathways play key roles in establishing a rapid and precise response at the infection site (Figure 1; Gu et al., 2017; Kulich et al., 2018; Tang et al., 2019). Examples are clathrin-mediated endocytosis of activated pattern recognition receptors (PRRs) and locally targeted, so-called polarized, exocytosis of antimicrobial compounds, cell wall material and PRRs (Ben Khaled et al., 2015).

During conventional exocytosis, proteins are transported from the ER through the Golgi and the trans-Golgi network/early endosomes (TGN/EE), where they are sorted to the 
plasma membrane and secreted in the extracellular space (Inada \& Ueda, 2014). In plants, the TGN/EE is a meeting point of exocytotic and endocytotic pathways (Viotti et al., 2010). Exocytosis is regulated by a complex molecular machinery, including the exocyst complex and SNARE proteins (Box II).

\section{Papillae formation}

One function of polarized exocytosis during defence is reinforcing the cell wall by the formation of papillae, or cell wall appositions, to halt pathogen penetration (Collinge, 2009; Voigt, 2014). Papillae consist mainly of callose but can also contain lignin, phenolic compounds and reactive oxygen species (ROS) (Collinge, 2009). Callose is a (1,3)- $\beta$-glucan polymer produced by callose synthase enzymes (Ellinger \& Voigt, 2014). Callose synthase 5, also known as PMR4 (Powdery Mildew Resistant4), is a transmembrane protein that accumulated at sites of attempted pathogen penetration where it locally deposits callose in the extracellular space (Ellinger et al., 2013). Overexpression of PMR4 in Arabidopsis thaliana resulted in complete penetration resistance against powdery mildew fungi due to a more strengthened cell wall (Ellinger et al., 2013; Eggert et al., 2014). Therefore, callose deposition is considered to be an important defence response against biotrophic fungi.

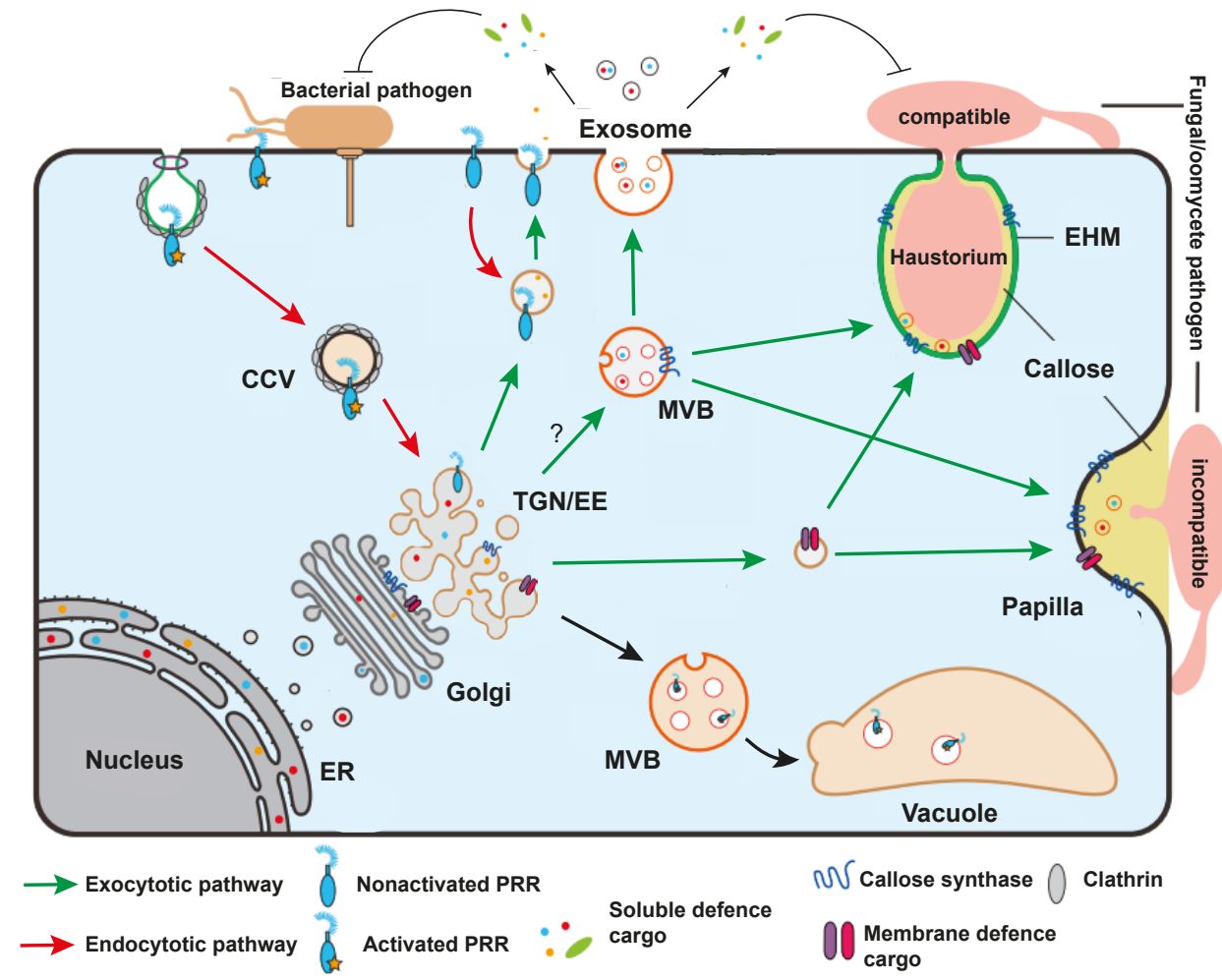

FIGURE 1. Membrane trafficking during plant defence.Schematic representation of exocytotic (green arrows) and endocytotic (red arrows) pathways involved in plant immunity. ER: endoplasmic reticulum, TGN/EE: transGolgi network/early endosomes, MVB: multivesicular body, CCV: clathrin-coated vesicle, EHM: extra-haustorial membrane. Adapted from Gu et al. (2017). 


\section{Box I: Plant immunity}

Plant-pathogen interactions can be either compatible or incompatible. During compatible interactions, pathogens successfully infect plants, while in incompatible interactions, pathogens are unable to infect plants. Pathogens can be categorized in three groups based on their infection strategies. Biotrophic pathogens need living plant cells for nutrient supply, while necrotropic pathogens actively induce necrosis in infected plant tissues and obtain nutrients form dead plant cells. Hemi-biotrophic pathogens show aspects of both infection strategies with an initial biotrophic phase followed by a necrotrophic phase.

According to the zigzag model that describes the arms race between plants and pathogens, plant immunity consists of two layers. The first layer is triggered when extracellular receptors on the plasma membrane, the so called pattern recognition receptors (PRRs), perceive conserved pathogen-associated molecular patterns (PAMPs) (Jones \& Dangl, 2006). This PAMP-triggered immunity (PTI) leads to dramatic reprogramming of the plant cell to prevent penetration of the pathogen, e.g. by the production of anti-microbial compounds and reactive oxygen species (ROS) and the deposition of callose in the form of papillae (Dodds \& Rathjen, 2010). The second layer of immunity is activated by pathogen-produced effectors that interfere with cellular processes to down-regulate PTI responses. These effectors are recognized by resistance $(\mathrm{R})$ proteins in the plant cell and this recognition leads to effector-triggered immunity (ETI). ETI is regulated by salicylic acid (SA) signalling and often manifested by localized cell death in the form of an hypersensitive response (HR) (Jones \& Dangl, 2006). This HR-mediated disease resistance is only effective against (hemi-) biotrophic pathogens. The discovery of effectors with typical PAMP characteristics and PAMPs resembling effectors, made it difficult to maintain a strict distinction between PTI and ETI (Thomma et al., 2011). Therefore, a new invasion model describing a continuous plant immune system was proposed (Cook et al., 2015). In this model, invasion patterns (PAMPs and effectors) are perceived by invasion pattern receptors (extracellular or intracellular) triggering a defence response and pathogens may use effectors to suppress this defence.

Most of the knowledge regarding the molecular components involved in membrane trafficking towards papillae comes from genes that were identified in genetic screens for mutations in $A$. thaliana that resulted in increased penetration by the non-adapted powdery mildew fungus Blumeria graminis (Yun \& Kwon, 2017). One gene identified in such a screen is PEN1 (Penetration 1) which encodes t-SNARE SYP121 (Collins et al., 2003). Since SYP121 accumulated at papillae and pen 1 mutants were delayed in papillae formation, Assaad et al. (2004) concluded that SYP121 is required for polarized exocytosis during papillae formation. SYP121 forms a SNARE-complex with t-SNARE SNAP33 and V-SNARE Vamp721/722, which also both accumulated at powdery mildew penetration sites in A. thaliana (Kwon et al., 2008). The fact that callose deposition is delayed and that vesicles containing PMR4 accumulated in SYP121 and Vamp721/722-silenced A. thaliana plants, indicates that Vamp721/722 vesicles transport callose synthases to pathogen penetration sites (Assaad et al., 2004; Kwon et al., 2008). 


\section{Haustoria formation}

Adapted pathogens that suppress early plant defence responses like papillae formation are able to successfully penetrate plant cells and establish a compatible interaction. During this interaction, biotrophic and hemi-biotrophic fungal and oomycete pathogens form specialized feeding structures, called haustoria, penetrating the plant cell wall. Haustoria are surrounded by a plant-derived membrane, the extra-haustorial membrane (EHM), which is connected to the plasma membrane and its formation requires refined manipulation of host membrane trafficking (Underwood, 2016). The discovery of plant proteins localizing to the EHM provided important insights in the biogenesis of the EHM. For example, resistance protein RPW8.2, that localized to the EHM in A. thaliana cells challenged by a powdery mildew fungus, was shown to be transported to the EHM by VAMP721/722-labelled exocytotic vesicles (Wang et al., 2009; Kim et al., 2014), suggesting that the exocytotic pathway is rerouted to the EHM.

An haustorial encasement is frequently observed in between the haustorium and the EHM. This is a layer of cell wall material such as callose that is secreted by host cells and thought to seal-off haustoria in non-host plants or mature haustoria in susceptible plants (Meyer et al., 2009; Wang et al., 2009; Lu et al., 2012; Faulkner, 2015). Haustorial encasements are formed by extended out-growth of papillae and likely by the same membrane trafficking pathways involved in papillae formation (Meyer et al., 2009).

\section{Unconventional exocytosis}

Besides the above described conventional exocytosis, also unconventional exocytosis plays an important role in plant immunity (Gu et al., 2017; Wang et al., 2017). One form of unconventional exocytosis is the direct fusion of multivesicular bodies (MVBs), also called late endosomes or prevacuolar compartments, with the plasma membrane (Wang et al., 2017). This leads to the formation of extracellular vesicles, so-called exosomes that are released in the apoplast (Gyorgy et al., 2011). In barley cells attacked by the powdery mildew fungus $B$. graminis MVBs released their exosomes into papillae at the site of infection (An et al., 2006; Nielsen et al., 2012). The finding that the t-SNAREs SYP121/PEN1 and SNAP33 resided in papillae of $A$. thaliana plants infected with the powdery mildew fungus Golovinomyces orontii, indicates the presence of membranous compartments reminiscent of exosomes, inside papillae (Meyer et al., 2009). This was confirmed by Rutter and Innes (2017) who purified extracellular vesicles from $A$. thaliana leaves infected with the bacterium Pseudomonas syringae and showed by proteomic analysis that t-SNARE SYP121 associates with these vesicles. Besides the importance of MVBs in papillae formation, MVBs are also suggested to be re-directed towards and fuse with the EHM. MVBs were found inside haustorial encasements of A. thaliana plants infected with the powdery mildew fungus $G$. orontii (Micali et al., 2011). In addition, Bozkurt et al. showed that a late endosome marker, Rab7 GTPase RabG3c, was present on the EHM of Nicotiana benthamiana plants infected with Phytophthora infestans, suggesting that vacuole-targeted late endosome trafficking which involves MVBs is re-directed towards the haustorial interface (Bozkurt et al., 2015). 


\section{Box II: Molecular regulation of exocytosis}

Exocytosis is a multistep process in which Golgi-derived membrane vesicles fuse with the plasma membrane facilitating membrane extension as well as the delivery of molecules to the extracellular space. The final steps of exocytosis involve tethering, docking and fusion of the secretory vesicles with the plasma membrane (A). These steps are tightly regulated by several multiprotein complexes.

The initial tethering of vesicles is mediated by tethering factors, e.g. the TRAPPII complex and the exocyst complex (Ravikumar et al., 2017). The exocyst complex is an evolutionary conserved octameric protein complex and consists of the following subunits: Sec3, Sec5, Sec6, Sec8, Sec10, Sec15, Exo70 and Exo84 (Munson \& Novick, 2006). The exocyst complex has been extensively studied in the budding yeast Saccharomyces cerevisiae, where it is involved in polarized exocytosis and cytokinesis (Mei \& Guo, 2018). In yeast, six of the eight exocyst subunits are attached to the vesicle, while Sec3 and Exo70 function as landmarks on the plasma membrane (B) (Mei \& Guo, 2018). The plant exocyst complex consists of the same eight subunits, but unlike yeast, plants contain multiple genes encoding a subunit (Cvrckova et al., 2012). This is best illustrated by the Exo70 family which is encoded by 23 paralogs in A. thaliana (Cvrckova et al., 2012). The plant exocyst complex plays a role in many developmental processes, including polarized exocytosis during tip growth, cytokinesis, secondary cell wall deposition, pollen acceptance and autophagy (Zarsky et al., 2013).

The tethered vesicle is tightly docked and subsequently fused with the plasma membrane by the SNARE (soluble N-ethylmaleimide-sensitive factor attachment protein receptor) complex (Duman \& Forte, 2003). One SNARE protein present on the vesicle (v-SNARE) and two or three SNARE proteins on the target membrane (t-SNAREs) need to match like locks-and-keys and assemble in a complex to facilitate vesicle fusion (Jahn \& Scheller, 2006). Unique sets of SNAREs provide specificity of vesicle fusion all through the cell endomembrane system, not only at the plasma membrane.
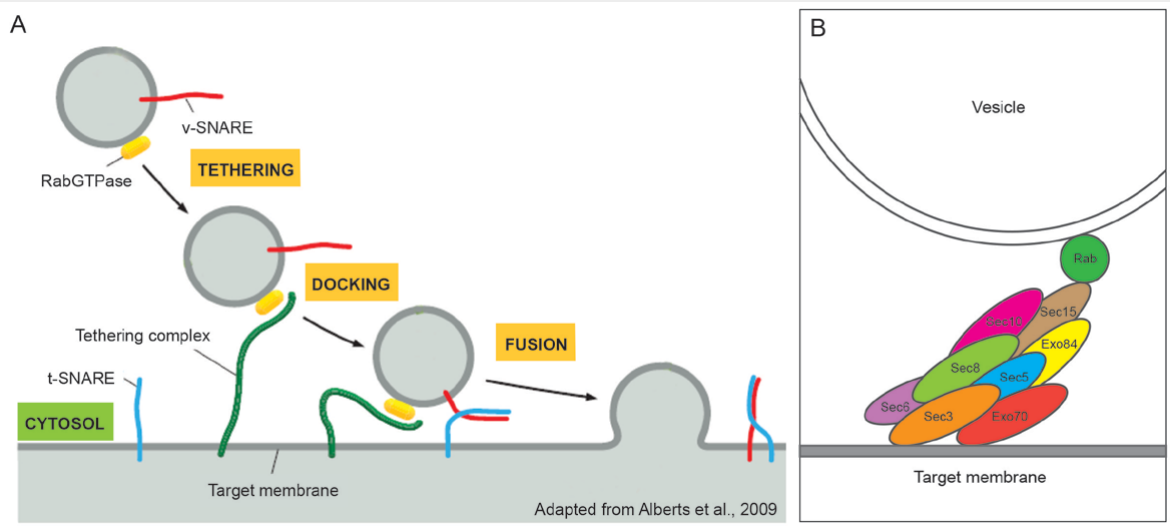
A possible source for MVBs is autophagy, a fundamental endomembrane trafficking route involved in protein degradation. In plants, autophagy plays a role in development, abiotic stress tolerance and immunity (Leary et al., 2018). Double membrane compartments, called autophagosomes, transport cargo to the vacuole for digestion (Pecenkova et al., 2017). In addition to this degradation pathway, autophagy has recently been suggested to mediate trafficking of phytochemicals (Pecenkova et al., 2017). To do so, autophagosomes might be re-routed and targeted to the cell wall via an exosome-like secretion pathway (Kulich \& Zarsky, 2014). It was shown that autophagosomes were re-directed towards the EHM of $P$. infestans and that they were important for polarized defence responses (Dagdas et al., 2018).

\section{The role of the exocyst in plant-microbe interactions}

The fact that polarized exocytosis is an integral component of plant defence, implies that also the exocyst complex could have an important role in plant immunity. The exocyst complex is an octameric protein complex involved in tethering secretory vesicles to the plasma membrane (Box II). Although increasing evidence shows that the exocyst complex is important for proper plant defence, studies have so far mainly focussed on one exocyst subunit family, i.e. Exo70 (Martin-Urdiroz et al., 2016).

A. thaliana Exo70B1 is the most frequently studied exocyst subunit in plant immunity. It was shown to be involved in the perception of pathogen-associated molecular patterns (PAMPs) like flg22, the conserved epitope of the bacterial flagellin (Stegmann et al., 2012). Exo70B1 mutants were more resistant to the oomycete Hyaloperonospora arabidopsidis and the powdery mildew fungus Golovinomyces cichoracearum (Stegmann et al., 2013; Zhao et al., 2015). With respect to the bacterial pathogen Pseudomonas syringae pv. tomato conflicting results were obtained. Stegmann et al. (2013) reported reduced resistance of Exo70B1 mutants, while Zhao et al. (2015) reported increased resistance against the same bacterial pathogen. Furthermore, A. thaliana Exo70B1 mutants older than six weeks showed an ectopic hypersensitive response (HR), also called a lesion mimic cell death response (Kulich et al., 2013; Stegmann et al., 2013). These lesion-mimic cell death responses of $A$. thaliana Exo70B1 mutants were also found in rice Sec3a mutants which resulted in enhanced resistance to the fungal pathogen Magnaporthe oryzae (Ma et al., 2018). Recently, Sabol et al. (2017) showed that A. thaliana Exo70B1 is recruited to the plasma membrane by RIN4, a well-known regulator of plant defence.

Expression of $A$. thaliana Exo70B2 and Exo70H1 is upregulated upon PAMP-treatment and mutants in either subunit are less resistant to $P$. syringae, $B$. graminis and $H$. arabidopsidis (Pecenkova et al., 2011; Stegmann et al., 2012). Exo70B2 is targeted for degradation by ubiquitin ligase PUB22, which is thought to regulate signalling upon PAMP-perception (Stegmann et al., 2012). In addition, Exo70F-silenced barley plants were more susceptible to B. graminis (Ostertag et al., 2013), and Sec5-silenced Nicotiana benthamiana plants were more susceptible to $P$. infestans (Du et al., 2015a). Together, this indicates that several exocyst subunits play a role in disease resistance. 


\section{Chapter 1}

The exocyst complex also seems to play a role in papillae formation and in the deposition of callose, the main component of papillae (Du et al., 2015a; Kulich et al., 2015; Guo et al., 2018b; Kulich et al., 2018). An A. thaliana Exo70B2 mutant formed abnormal papillae surrounded with a dense halo of vesicle-like compartments in response to $B$. graminis infection (Pecenkova et al., 2011). A. thaliana Exo70H4 mutants were impaired in callose deposition in trichomes and upon PAMP-treatment which is likely caused by defects in exocytosis of callose synthase PMR4 (Kulich et al., 2015; Kulich et al., 2018). PMR4 colocalized with Exo70H4 in plasma membrane micro-domains that fail to develop in the Exo70H4 mutants (Kulich et al., 2018). In rice, the Bhp6 resistance protein that confers broad resistance to planthoppers, interacted with Exo70E1 and this interaction might increase callose deposition in phloem cells to prevent feeding by planthoppers (Guo et al., 2018b). Furthermore, Sec5-silenced N. benthamiana plants showed less callose deposition upon bacterial infection and less exocytosis of the defence-related protein PR1 to the apoplast upon salicylic acid treatment (Du et al., 2015a). Together, these results suggest that the exocyst complex is involved in polarized exocytosis during defence.

Besides the full exocyst complex, also exocyst subcomplexes have been found. For example in mammalian cells, exocyst subunits Sec5, Sec6, Sec8 and Exo70 are part of an exocyst subcomplex involved in autophagy and innate immune signalling (Tan et al., 2015). In $A$. thaliana, the exocyst subcomplex consisting of Sec5, Exo70B1 and Exo84 was shown to be involved in autophagy-related transport of anthocyanins to the vacuole (Kulich et al., 2013; Pecenkova et al., 2017). Since autophagy is an important process in plant immunity (Leary et al., 2018), this implies another function for exocyst subunits in plant defence.

Similar to biotrophic plant pathogens, beneficial plantmicrobes, like arbuscular mycorrhizas and nitrogen-fixing bacteria, enter the plant cell and have intimate relationships with their hosts. Also these beneficial plant-microbe interactions depend on specialized interfaces between plant cells and microbes and the formation of these interfaces likely involves exocytosis. Indeed, the exocyst complex was found to be involved in several beneficial plant-microbe interactions (Genre et al., 2012; Zhang et al., 2015; Gavrin et al., 2017). During Medicago-arbuscular mycorrhizal interactions, several exocytotic markers localized to tips of arbuscular branches including exocyst subunits Exo70 $\mathrm{i}$ and Exo84b (Genre et al., 2012; Zhang et al., 2015; Gavrin et al., 2017). In addition, Exo70i is required for proper interface development and mycorrhizal growth inside the host cell (Zhang et al., 2015). In a similar way, Exo70i is recruited to tips of growing infection threads during Medicago-rhizobium interactions (Gavrin et al., 2017). Concluding, these studies suggest that the exocyst complex has an important function in remodelling membranes to accommodate microbes. However, actual localization of the exocyst at sites of microbial contact has only been visualized for beneficial microbes and not yet upon infection with pathogenic microbes. 


\section{Effectors targeting host membrane trafficking}

The importance of membrane trafficking in plant defence is further illustrated by the fact that several pathogen effectors target components of the membrane trafficking machinery (Table 1). Khan et al. (2018) surveyed the literature on effector targets and showed that $5 \%$ of the known oomycete effectors and $13 \%$ of the known fungal effectors target regulators of plant subcellular transport.

The plant cytoskeleton is targeted by several pathogen effectors. The actin cytoskeleton is for example disrupted by P. syringae effector HopW1 (Kang et al., 2014). This actin disruption inhibits endocytosis and trafficking to the vacuole. P. syringae effectors HopE1 and HopZ1a disrupts microtubule organization, thereby inhibiting the exocytotic pathway which, in turn, results in reduced callose deposition and PR1 secretion (Lee et al., 2012; Guo et al., 2016). In addition, kinesins which are (microtubule) cytoskeleton-associated motor proteins are targeted by bacterial and oomycete effectors (Mukhtar et al., 2011; Shimono et al., 2016).

There are also pathogen effectors known that target endocytosis. Large screens revealed for example that several oomycete and fungal effectors interact with clathrin heavy chains (Mukhtar et al., 2011; Wessling et al., 2014; K. Bouwmeester, personal communication). In addition, the $P$. infestans RXLR effector AVR3a is known to associate with dynamin-related protein 2 (DRP2), which is a GTPase involved in regulating endocytosis (Chaparro-Garcia et al., 2015).

Finally, also exocytosis, and specifically the exocyst complex, is targeted by pathogen effectors. It was shown for example, that the fungal effector AVR-Pii from the rice blast fungus M. oryzae interacts with rice Exo70F2 and -F3 (Fujisaki et al., 2015), and that RXLR effector AVR1 from the oomycete pathogen $P$. infestans interacts with potato Sec5 (Du et al., 2015a). Via this interaction AVR1 can suppress Sec5-mediated callose deposition and other host defence responses (Du et al., 2015a). Sec5 seems to accumulate in the presence of AVR1 suggesting that AVR1 stabilizes Sec5. Furthermore, yeast-two-hybrid $(\mathrm{Y} 2 \mathrm{H})$ screens revealed other effectors that potentially target exocyst subunits including RxL62 and RxL470 from the oomycete $H$. arabidopsidis and HopC1 from the bacterium $P$. syringae interacting with $A$. thaliana Sec5a (Wessling et al., 2014), and P. infestans RXLR effector IPI-O with potato Sec10 (K. Bouwmeester, personal communication). Yet another RXLR effector, i.e. Phytophthora brassicae effector RxLR24 that targets different members of the RabA GTPase family of $A$. thaliana and potato, was found to inhibit exocytosis of antimicrobial compounds (Tomczynska et al., 2018). Together, these studies show that membrane trafficking and specifically the exocyst, are convergent hubs targeted by multiple pathogen effectors to suppress plant defence. 
TABLE 1. Pathogen effectors targeting host membrane trafficking.

\begin{tabular}{|c|c|c|c|c|}
\hline Pathogen $^{a}$ & Effector & Host target & Assayb $^{b}$ & Reference $^{c}$ \\
\hline \multicolumn{5}{|l|}{ Bacteria } \\
\hline P. syringae & HopC1 & Exocyst subunit Sec5A & $\mathrm{Y} 2 \mathrm{H}$ & 1 \\
\hline P. syringae & HopC1 & Kinesin motor protein & $\mathrm{Y} 2 \mathrm{H}$ & 1 \\
\hline P. syringae & HopG1 and HopZ & Kinesin motor protein & $\mathrm{Y} 2 \mathrm{H}, \mathrm{Co}-\mathrm{IP}$ & $1 ; 2$ \\
\hline P. syringae & HopM1 & AtMIN7/BEN1 (ARF-GEF) & $\mathrm{Y} 2 \mathrm{H}, \mathrm{Co}-\mathrm{IP}$ & $3 ; 4$ \\
\hline P. syringae & HopW1 & Actin & $\mathrm{Y} 2 \mathrm{H}$ & 5 \\
\hline P. syringae & HopZ1a & Tubulin & Co-IP & 6 \\
\hline P. syringae & AvrPto & $\begin{array}{l}\text { Rab8-related GTP-binding } \\
\text { protein }\end{array}$ & $\mathrm{Y} 2 \mathrm{H}$ & 7 \\
\hline P. syringae & HopE1 & MAP65 & $\mathrm{BiFC}, \mathrm{Y} 2 \mathrm{H}, \mathrm{Co}-\mathrm{IP}$ & 8 \\
\hline X. campestris & AvrBsT & ACIP1 & $\mathrm{Y} 2 \mathrm{H}, \mathrm{Co}-\mathrm{IP}$ & 9 \\
\hline \multicolumn{5}{|l|}{ Fungi } \\
\hline G. orontii & OEC45 and 65 & Clathrin heavy chain 2 & $\mathrm{Y} 2 \mathrm{H}$ & $1 ; 10$ \\
\hline G. orontii & OEC65 & PRA1.F1-3 & $\mathrm{Y} 2 \mathrm{H}$ & 1 \\
\hline B. graminis & BEC4 & HvARF-GAP & $\mathrm{Y} 2 \mathrm{H}, \mathrm{BiFC}$ & 11 \\
\hline M. oryzae & AVR-Pii & $\begin{array}{l}\text { Exocyst subunits Exo70F2 and } \\
\text { Exo70F3 }\end{array}$ & $\mathrm{Y} 2 \mathrm{H}, \mathrm{Co}-\mathrm{IP}$ & 12 \\
\hline \multicolumn{5}{|l|}{ Oomycetes } \\
\hline P. infestans & AVR1 & Exocyst subunit Sec5a & Y2H, Co-IP, BiFC & 13 \\
\hline P. infestans & AVR1 & Clathrin heavy chain & $\mathrm{PD}$ & 14 \\
\hline P. infestans & AVR1 & Actin & PD & 14 \\
\hline P. infestans & AVR3a & $\mathrm{NbDRP2}$ & Co-IP & 15 \\
\hline P. infestans & $\mathrm{IPI}-\mathrm{O}$ & Exocyst subunit Sec10 & $\mathrm{PD}$ & 14 \\
\hline P. infestans & PexRD54 & Autophagy protein ATG8CL & Co-IP & 16 \\
\hline P. brassicae & $\mathrm{RxLR24}$ & RABA GTPases & Co-IP & 17 \\
\hline H. arabidopsidis & 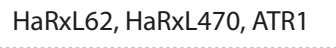 & Exocyst subunit Sec5a & $\mathrm{Y} 2 \mathrm{H}$ & $1 ; 10$ \\
\hline H. arabidopsidis & HaRxL73, HaRxL515, ATR13 & Clathrin heavy chain 2 & $\mathrm{Y} 2 \mathrm{H}$ & $1 ; 10$ \\
\hline H. arabidopsidis & $\mathrm{HaRxL73,} \mathrm{HaRxL79,} \mathrm{ATR13}$ & Kinesin motor protein & $\mathrm{Y} 2 \mathrm{H}$ & 1 \\
\hline H. arabidopsidis & $\mathrm{HaRxL74}$ & PRA1.F3 & $\mathrm{Y} 2 \mathrm{H}$ & $1 ; 10$ \\
\hline H. arabidopsidis & $\mathrm{HaRxL} 470$ & Remorin 6.4 & $\mathrm{Y} 2 \mathrm{H}$ & 1 \\
\hline H. arabidopsidis & $\mathrm{HaRxL470,} \mathrm{ATR13}$ & $\begin{array}{l}\text { Ypt/Rab-GAP domain of Gyp1p } \\
\text { superfamily protein }\end{array}$ & $\mathrm{Y} 2 \mathrm{H}$ & 1 \\
\hline
\end{tabular}

a Pathogens: Pseudomonas syringae, Xanthomonas campestris, Golovinomyces orontii, Blumeria graminis, Magnaporthe oryzae, Phytophthora infestans, and Hyaloperonospora arabidopsidis.

${ }^{\mathrm{b}}$ Assays used to reveal effector-target interactions: bimolecular fluorescence complementation assay (BiFC), coimmunoprecipitation (Co-IP), pulldown (PD) and yeast-two-hybrid (Y2H).

'References: 1 Mukhtar et al., 2011; 2 Shimono et al., 2016; 3 Nomura et al., 2006; 4 Nomura et al., 2011 ; 5 Kang et al., 2014; 6 Lee et al., 2012; 7 Bogdanove \& Martin, 2000; 8 Guo et al., 2016; 9 Cheong et al., 2014; 10 Wessling et al., 2014; 11 Schmidt et al., 2014; 12 Fujisaki et al., 2015; 13 Du et al., 2015a; 14 K. Bouwmeester, personal communication; 15 Chaparro-Garcia et al., 2015; 16 Dagdas et al., 2016; 17 Tomczynska et al., 2018. 


\section{Scope of this thesis}

In this thesis, we used a microscopy-based approach to understand the role of polarized exocytosis, and especially the exocyst complex, in plant defence. Figure 2 shows a schematic overview of the research objectives of Chapters 2-5.

In Chapter 2, we performed a large phytopathological screen to get a complete overview of the contribution of different exocyst subunits to plant immunity. We used virus-induced gene-silencing in $N$. benthamiana to downregulate the exocyst subunits. The effect of this downregulation on plant susceptibility was subsequently determined with disease assays using pathogens with different lifestyles, i.e. the (hemi)-biotrophic pathogens $P$. infestans and $P$. syringae and the necrotrophic pathogen Botrytis cinerea. We also tested the role of $N$. benthamiana exocyst subunits in callose deposition upon bacterial pathogen attack.

To address the function of the exocyst complex in defence at a cellular level, we aimed to visualize exocyst-mediated exocytosis in living cells upon pathogen attack. However, livecell imaging of plant-pathogen interactions is often hampered by the tissue complexity and multi-cell layered nature of plants. Therefore, in Chapter 3, we developed a novel model system to study cellular plant defence. We made use of the moss Physcomitrella patens, which single-cell layered morphology is ideal for high resolution microscopy, and the oomycete pathogen Phytophthora (Box III). We explored the ability of several Phytophthora species to infect $P$. patens and also defence responses induced upon pathogen infection were analysed. We developed a novel imaging set-up and demonstrated the high potential of this Physcomitrella-Phytophthora pathosystem for live-cell imaging of cellular defence by visualizing local reorganisation of the actin cytoskeleton. This system is used in Chapter $\mathbf{4}$ and Chapter $\mathbf{5}$ to address questions related to the localization of exocyst subunits during infection and the role of the $P$. infestans effector AVR1 in suppressing exocyst functioning.

The aim of Chapter 4 was to study the subcellular localization of $P$. patens exocyst subunits Sec3, Sec5 and Sec6 upon infection with Phytophthora. Homologous recombination was used to introduce DNA fragments encoding fluorescent tags into the endogenous exocyst subunit genes. The intensity of the locally deposited autofluorescent material was higher than that of the GFP-tagged exocyst subunits, making it technically challenging to visualize exocyst subunit localization during infection. We explored the possibilities to eliminate this undesired autofluorescence and developed a set-up using time-gated fluorescent microscopy to efficiently do so. Hence we were able to show exocyst subunit accumulation on membranes surrounding papilla-like structures and hyphal encasements.

The aim of Chapter $\mathbf{5}$ was to deepen our mechanistic understanding of how pathogen effectors manipulate exocyst functioning in order to suppress plant defence. We focussed on the interaction between P. infestans effector AVR1 and P. patens exocyst subunit Sec5. Transgenic $P$. patens lines were generated with inducible expression of $A V R 1$ to investigate the effect of this RXLR effector on $P$. patens growth and Sec5b or Sec5d localization. In addition, phenotypic defects of AVR1-expressing $P$. patens plants were compared with phenotypes of Sec5- or Sec6-silenced P. patens lines. 


\section{Chapter 1}

Finally, in Chapter 6, the findings of this thesis are discussed in a broader perspective. I elaborate on the pros and cons of the use of $P$. patens as a model system for imaging cellular defence processes and formulate hypotheses on the regulation of polarized exocytosis and the function of the exocyst in plant immunity.

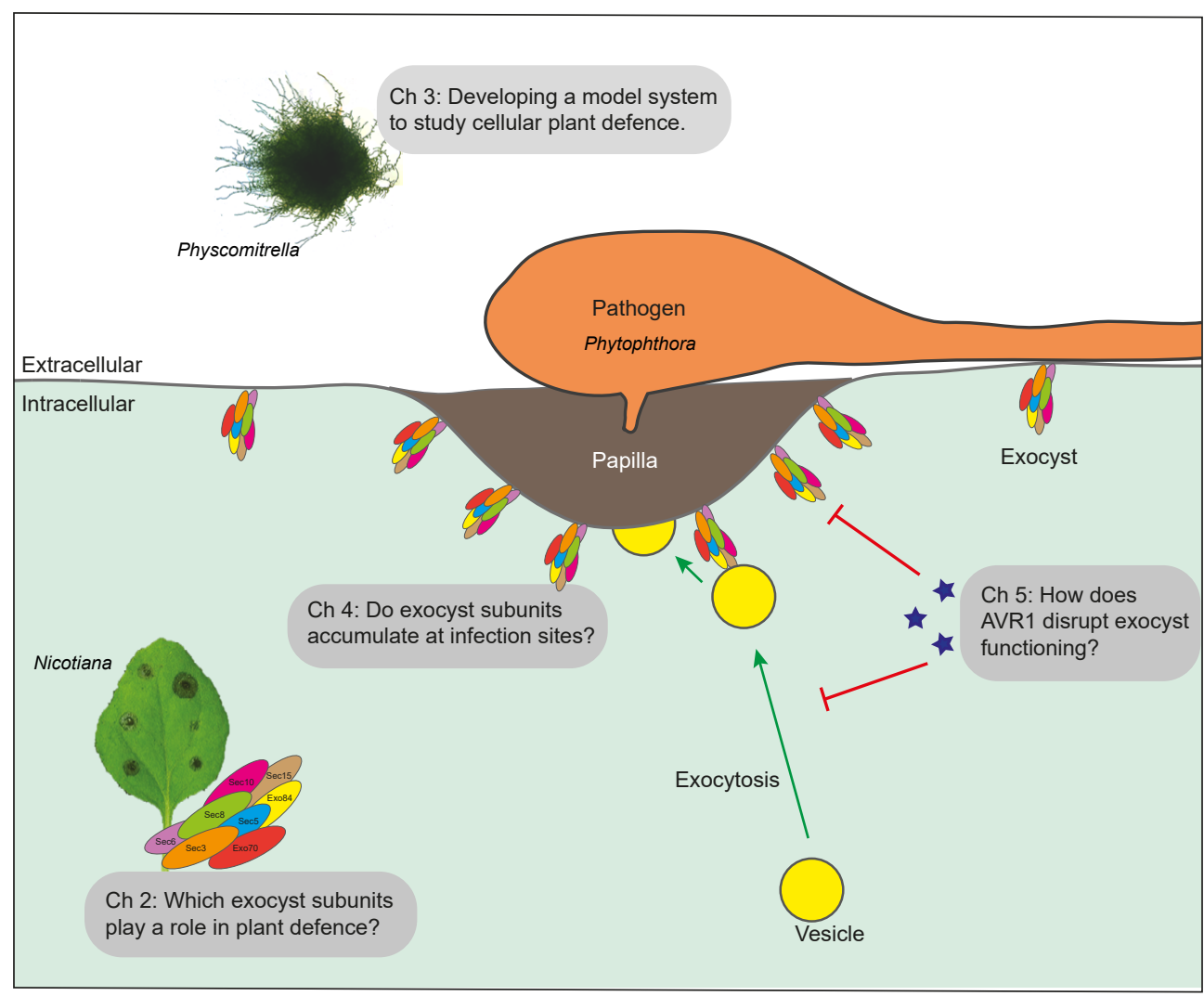

FIGURE 2. Schematic overview of the objectives of the indicated chapters (Ch). Drawing not to scale. 


\section{Box III: Key actors in this thesis}

\section{Phytophthora}

Phytophthora species belong to the oomycetes, which is a diverse group of organisms showing a fungal-like morphology. They are classified as straminopiles, an evolutionary lineage that also includes brown algae and diatoms (Kamoun et al., 2015). The Phytophthora genus contains over 100 species that are all plant pathogens (Kroon et al., 2012). Notorious species are Phytophthora infestans, the causal agent of potato and tomato late blight, and Phytophthora capsici, which has a broad host range and infects amongst others tomato and pepper (Fry, 2008; Kamoun et al., 2015). During the disease-cycle, Phytophthora zoospores land on a leaf, encyst and germinate. The tip of the germ tube forms an appressorium that enables host cell penetration. Subsequently, invasive hyphae colonize the mesophyll cell layer and produce feeding structures called haustoria. Haustoria are thought to deliver effectors into plant cells and to facilitate uptake of nutrients. P. infestans and $P$. capsici are hemi-biotrophic pathogens that need living plant tissue for the initial infection phases, but plant cells die when the lesion expands. In this final phase, sporangia are produced which can start a new infection-cycle by either germinating directly or releasing motile zoospores (Judelson \& Blanco, 2005).

\section{Physcomitrella patens}

P. patens is a moss that forms an evolutionary link between green algae and vascular plants (Knight et al., 2009). It has become an important model system for plant cell biology. Advantages include easy culturing, highly efficient gene targeting by homologous recombination, a sequenced genome, and not in the least the fact that most tissues are only one cell layer thick and ideal for microscopy (Nishiyama et al., 2000; Rensing et al., 2008). Haploid spores germinate and form a filamentous network called protonema. This protonema consists of two types of cells: chloronema cells containing large chloroplasts and mainly involved in photosynthesis, while the faster-growing caulonemal cells with fewer plastids are involved in substrate colonization and nutrient acquisition (Menand et al., 2007). In the next phase of the $P$. patens life-cycle, protonema cells differentiate into leafy gametophores on top of which diploid sporophytes are formed that can again form haploid spores (Cove, 2005). In recent years several studies have shown that $P$. patens can host various types of pathogens and that it shares conserved defence responses with seed plants (Ponce de Leon \& Montesano, 2017). As a result, P. patens is emerging as a model organism to study plant immunity. 



\section{Chapter 2}

Solanaceous exocyst subunits are involved in immunity to diverse plant pathogens

Yu Dü

Elysa J.R. Overdijk"

Jeroen A. Berg

Francine Govers

Klaas Bouwmeester

\# These authors contributed equally 


\section{Abstract}

The exocyst, a multi-protein complex consisting of eight subunits, plays an essential role in many biological processes by mediating secretion of postGolgi- derived vesicles towards the plasma membrane. In recent years roles for plant exocyst subunits in pathogen defence have been uncovered, largely based on studies in the model plant Arabidopsis thaliana. Only a few studies have been undertaken to assign the role of exocyst subunits in plant defence in other plants species, including crops. In this study, predicted protein sequences from exocyst subunits were retrieved by mining databases from the Solanaceous plants Nicotiana benthamiana, tomato and potato. Subsequently, their evolutionary relationship with $A$. thaliana exocyst subunits was analysed. Gene silencing in $N$. benthamiana showed that several exocyst subunits are required for proper plant defence against the (hemi-)biotrophic plant pathogens Phytophthora infestans and Pseudomonas syringae. In contrast, some exocyst subunits seem to act as susceptibility factors for the necrotrophic pathogen Botrytis cinerea. Furthermore, the majority of the exocyst subunits were found to be involved in callose deposition, suggesting that they play a role in basal plant defence. This study provides insight into the evolution of exocyst subunits in Solanaceous plants and is the first to show their role in immunity against multiple unrelated pathogens. 


\section{Introduction}

The exocyst, an evolutionary conserved multi-protein complex that consists of eight subunits, is involved in directing post-Golgi-derived vesicles towards the plasma membrane (Munson \& Novick, 2006). This process works one step before docking and fusion of vesicles at the membrane mediated by the soluble SNARE-complex (Duman \& Forte, 2003). The exocyst complex was first discovered in yeast and shown to be required for secretion and polarized growth during budding (TerBush \& Novick, 1995). Later on, homologues of the eight exocyst subunits, namely Sec3, Sec5, Sec6, Sec8, Sec10, Sec15, Exo70 and Exo84, were identified in higher organisms (Ting et al., 1995; Hsu et al., 1996; Kee et al., 1997; Brymora et al., 2001; Matern et al., 2001). In yeast and mammals interaction between the individual exocyst subunits was studied by different methods and this revealed that each exocyst subunit can associate with multiple other subunits in the complex (Munson \& Novick, 2006). Studies using chromatographic fractionation and yeast two-hybrid assays showed that also in plants the exocyst is a true protein complex and that the pairwise interactions between the exocyst subunits are conserved resembling those in yeast and animals (Hala et al., 2008; Zhang et al., 2010; Zarsky et al., 2013; Zhang et al., 2013). Unlike yeast and animals, which have single-copy genes for each exocyst subunit, plants often have two or more genes encoding the same subunit, for example A. thaliana has three Exo84 and 23 Exo70 paralogues (Synek et al., 2006; Chong et al., 2010; Cvrckova et al., 2012).

So far, several studies have shown that exocyst subunits have a role in plant growth and development. For example, multiple exocyst subunits were reported to be required for proper cytokinesis (Fendrych et al., 2010; Rybak et al., 2014). It was also suggested that the exocyst complex is crucial for polar exocytosis during growth of pollen and roots (Cole et al., 2005; Synek et al., 2006; Hala et al., 2008; Cole et al., 2014; Bloch et al., 2016; Tan et al., 2016). Furthermore, studies showed that the exocyst complex is required for a broad variety of developmental processes, including pollen acceptance (Samuel et al., 2009; Safavian et al., 2015), secondary cell wall deposition (Li et al., 2013; Kulich et al., 2015; Oda et al., 2015; Vukasinovic et al., 2017), PIN-protein recycling (Drdova et al., 2013; Tan et al., 2016), stomatal opening (Hong et al., 2016; Seo et al., 2016), and autophagy (Kulich et al., 2013).

In plants, vesicle trafficking is an integral component of the immune mechanism and plays an important role in defence against pathogens by accommodating secretion of antimicrobial compounds to infection sites to hamper pathogen colonization. For example, infection of barley with the powdery mildew fungus Blumeria graminis leads to a cell wallassociated defence that is accompanied by accumulation of multi-vesicular bodies that participate in cell wall apposition, and reactive oxygen species (ROS) accumulation at the cell periphery (An et al., 2006). In addition, penetration resistance of Arabidopsis thaliana to $B$. graminis was shown to be mediated by the syntaxin SYP121, a component of the SNARE-complex (Collins et al., 2003). Moreover, induction of systemic acquired resistance was reported to be dependent on NPR1-regulated expression of several secretion-related genes (Wang et al., 2005). 
Emerging evidence shows that the exocyst complex is an additional key player in plantpathogen interactions. This is mostly based on studies that use Exo70 mutant plants (Martin-Urdiroz et al., 2016). A. thaliana Exo70B1 mutants show lesion-mimic cell death mediated by salicylic acid accumulation (Kulich et al., 2013; Stegmann et al., 2013). A. thaliana ExO70B1 is required for autophagy, a process involved in degradation and recycling of cellular components, and plays an important role in receptor signalling during plant defence (Kulich et al., 2013). Zhao et al. (2015) showed that Exo70B1 interacts with TIR-NBS2, an atypical nucleotide-binding domain and leucine-rich repeat-containing (NLR) resistance protein that lacks the leucine-rich repeat domain, and that disruption of this interaction leads to constitutive activation of defence. Recently, Sabol et al. (2017) showed that $A$. thaliana Exo70B1 also interacts with RIN4, a well-known regulator of plant defence that is cleaved by the bacterial protease AvrRpt2 of Pseudomonas syringae (Afzal et al., 2011). Hence, it was suggested that cleavage of RIN4 releases Exo70B1 from the plasma membrane and thereby suppresses tethering of defence-related vesicles (Sabol et al., 2017). Barley Exo70F-like, and A. thaliana Exo70B2 and Exo70H1 were reported to be involved in basal resistance against $B$. graminis (Pecenkova et al., 2011; Ostertag et al., 2013). A. thaliana Exo70B2, the target of the ubiquitin ligase PUB22, was found to be required for full activation of immune signalling elicited by defence pathogen-associated molecular patterns (PAMPs) (Stegmann et al., 2012). Pečenková et al. (2011) found that A. thaliana EXO70B2 mutants develop aberrant papillae with halos and show enhanced penetration by B. graminis. Besides Exo70B2, the SNARE syntaxin PENETRATION1 (PEN1), is also required for timely assembly of papillae and focal secretion, and thus for blocking penetration by B. graminis (Collins et al., 2003). Penetration resistance is often conferred by cell wall thickening of which callose is a major component (Collinge, 2009). In Nicotiana benthamiana, silencing of Sec5 impairs callose deposition (Du et al., 2015a) and in A. thaliana, Exo70H4 was found to be important for callose deposition in trichomes (Kulich et al., 2015). As such it can be hypothesized that the exocyst is mediating callose deposition at infection sites. Exocyst subunits were also reported to play an important role during arbuscular mycorrhizal symbiosis, suggested by their accumulation at sites of microbial contact and role in the formation of the perifungal membrane (Genre et al., 2012; Zhang et al., 2015).

The importance of vesicle trafficking and exocytosis in plant immunity was also shown by the fact that plant pathogens secrete effectors to target vesicle trafficking-related proteins. Examples are the bacterial pathogen P. syringae that uses its effector HopM1 to destabilize AtMIN7, a key regulator of vesicle formation, to facilitate infection of A. thaliana (Nomura et al., 2006; Nomura et al., 2011), and the fungal plant pathogen Alternaria carthami that secretes the phytotoxin brefeldin A (BFA) to inhibit Golgi-derived vesicle formation (Driouich et al., 1997). Furthermore, it was shown that the effector AVRPii from Magnaporthe oryzae targets rice Exo70F2 and -F3 (Fujisaki et al., 2015), and that the oomycete Phytophthora infestans exploits the RXLR-effector AVR1 for targeting the exocyst subunit Sec5 in potato to suppress host defence (Du et al., 2015a). 
In general, the role of the exocyst complex in plant defence has not been studied in depth. The knowledge obtained so far is rather fragmented and covers only a few of the Exo70 paralogues and two other exocyst subunits, namely Sec5 and Exo84B. The aim of this study was to investigate the role of exocyst subunits in plant immunity of the Solanaceous model plant $N$. benthamiana. Genes encoding the exocyst subunits were identified in Solanaceous plants and phylogenetic analyses were performed to study their relationship with exocyst subunits of $A$. thaliana. We then employed Tobacco tattle virus (TRV)-mediated virus-induced gene silencing (VIGS) in N. benthamiana and tested the effect of silenced exocyst subunit genes in disease assays. The results show that multiple Solanaceous exocyst subunits play a role in defence against plant pathogens with different lifestyles.

\section{Materials and methods}

\section{Gene identification and phylogenetic analysis}

Protein sequences of the $A$. thaliana exocyst subunits were retrieved from the TAIR database using the gene inventory of Chong et al. (2010). BLAST analysis was subsequently performed against the genomes and predicted proteomes of $N$. benthamiana, Solanum tuberosum (potato) and Solanum lycopersicum (tomato) at the Sol Genomics Network (SGN) website (http://solgenomics.net). Protein sequences are listed in Supplementary Table $\mathrm{S} 1$ at JXB online.

Protein sequence alignments were constructed using ClustalW with default settings (protein weight matrix GONNET, gap opening of 10, gap extension of 0.2 ). Obtained sequence alignments were used as input to reconstruct phylogenetic trees using the Neighbor Joining algorithm in MEGA5 with 5000 bootstrap replicates. Branches corresponding to partitions reproduced in $<50 \%$ of bootstrap replicates were collapsed.

\section{Plasmid construction}

Multi-sequence alignments by ClustalW or MultAlin were used to pinpoint gene segments containing stretches of $>25$ nucleotides with $100 \%$ identity to the target gene. The specificity of gene silencing was verified by BLAST analysis and the VIGS web-tool at the SGN website. Gene segments containing either EcoRI/Sacl or BamHI/Sacl restriction sites were synthesized by Eurofins Genomics (Supplementary Table S2), and cloned into the binary vector pTRV2. Binary plasmids were transformed to Agrobacterium tumefaciens strain AGL1 via electroporation. TRV-constructs were generated to silence the $N$. benthamiana $\mathrm{Sec}$ and Exo genes in the various clades and subclades (Supplementary Table S2). Four of the silencing constructs contain multiple target sequences in order to silence all genes in the subclades Exo70C, Exo70D, Exo70G, and Exo70H, respectively. 


\section{Chapter 2}

\section{Plant material and pathogen growth}

Nicotiana benthamiana was grown in potting soil under standardized greenhouse conditions. Phytophthora infestans isolate 14-3-GFP was grown in the dark on rye sucrose agar medium at $18^{\circ} \mathrm{C}$. $P$. infestans zoospores were isolated according to Champouret et al. (2009) and the concentration was adjusted to $1 \times 10^{5}$ zoospores $\mathrm{ml}^{-1}$. N. benthamiana leaves were detached, placed in trays and inoculated at the abaxial leaf surface with $10 \mu \mathrm{l}$ droplets of a $P$. infestans zoospore suspension. Inoculated leaves were incubated at high humidity at $18^{\circ} \mathrm{C}$ in the dark for the first $24 \mathrm{~h}$ followed by a $16 \mathrm{~h}$ photoperiod. Lesion diameters were measured 6 days after inoculation (dai). Average lesion areas were determined as previously described (Vleeshouwers et al., 1999), and normalized to those of TRV:GUS-treated control plants.

Botrytis cinerea isolate B05.10 was cultured on malt extract agar medium at $20^{\circ} \mathrm{C}$ and sporulation was induced by UV light. Conidia were harvested from sporulating plates, resuspended in sterile water and filtered through cheesecloth. Conidia were washed twice and resuspended in potato dextrose broth (PDB; $12 \mathrm{~g} \mathrm{l}^{-1}$ ). $N$. benthamiana plants were inoculated with $2 \mu \mathrm{l}$ droplets of $1 \times 10^{6}$ conidia $\mathrm{ml}^{-1}$ and placed in closed transparent boxes at room temperature. Plants were kept in the dark for the first $24 \mathrm{~h}$. Lesion diameters were measured 3 dai, and those expanding $3.5 \mathrm{~mm}$ were scored as secondary lesions.

Pseudomonas syringae was cultured on King's B medium with rifampicin (100 $\mu \mathrm{g} \mathrm{ml}$ $\left.{ }^{1}\right)$ at $28^{\circ} \mathrm{C}$. Inoculum of $P$. syringae pv. syringae isolate B728a (Pss) was prepared from an overnight culture that was resuspended in $10 \mathrm{mM} \mathrm{MgCl}_{2}$ to an OD of 0.01 [7x10 colonyforming units (cfu) $\mathrm{ml}^{-1}$ ] and sprayed on detached $N$. benthamiana leaves placed in closed trays that were kept at $21^{\circ} \mathrm{C}$ for $3 \mathrm{~d}$. To quantify the total amount of bacteria, leaf samples were ground in $10 \mathrm{mM} \mathrm{MgCl}{ }_{2^{\prime}}$ and colony-forming units were counted after dilution plating. For infiltration of Pseudomonas syringae pv. tomato (Pst) $\Delta \mathrm{hrcC}$, an overnight culture was collected and resuspended in $10 \mathrm{mM} \mathrm{MgCl}_{2}$. The inoculum concentration used for infiltration was set to an OD600 of $0.5\left(\sim 1.0 \times 10^{8} \mathrm{cfu} \mathrm{ml}^{-1}\right)($ Kim et al., 2011).

\section{Agroinfiltration and virus-induced gene silencing}

Agrobacterium tumefaciens strains containing binary TRV-vectors were grown in medium containing the appropriate antibiotics at $28{ }^{\circ} \mathrm{C}$. Agrobacterium cultures were centrifuged and resuspended in infiltration buffer [per litre: $10 \mathrm{mM} \mathrm{MES} \mathrm{pH} \mathrm{5.6,} 5 \mathrm{~g}$ Murashige and Skoog (MS) salts (without vitamins), $20 \mathrm{~g}$ of sucrose, and $150 \mu \mathrm{M}$ of acetosyringone]. Agrobacterium strains harbouring PTRV2 derivatives or PTRV1 were mixed in a 1:1 ratio (at a final $\mathrm{OD}_{600}$ of 1.0), and VIGS was performed on 16-day-old N. benthamiana by agroinfiltration into the first two emerging leaves (Peart et al., 2002). TRV:GUS and TRV:SGT1 were included as controls (Tameling \& Baulcombe, 2007). The fifth and sixth leaf were harvested 3-4 weeks after agroinfiltration, and subsequently used for further analysis. 


\section{Quantitative RT-PCR}

Total RNA was isolated from N. benthamiana leaves by a NucleoSpin RNA plant mini-kit (Clontech). cDNA synthesis was performed on $1 \mu \mathrm{g}$ of total plant RNA using an oligo (dT) primer and M-MLV reverse transcriptase (Invitrogen). Quantitative reverse transcriptionPCR (qRT-PCR) was performed using SYBR Green master mix (Promega), gene-specific primers (Supplementary Table S3), and $2 \mu$ of 10-fold diluted cDNA using a Bio-Rad 7300 thermocycler. Gene expression levels were normalized to Actin expression (Gabriels et al., 2007).

\section{Callose deposition assays}

Nicotiana benthamiana leaf discs collected $14 \mathrm{~h}$ after infiltration with Pst DC3000 $\Delta \mathrm{hrcC}$, were cleared with ethanol and stained with aniline blue $(1 \%, w / v)$. Callose deposition was visualized using epifluorescence microscopy as previously described (Bouwmeester et al., 2011). Four or five leaf disks were examined for every sample, and three microscope pictures $\left(3.5 \mathrm{~mm}^{2}\right)$ were taken randomly per leaf disk. The total numbers of callose spots were counted per microscope picture.

\section{Results and discussion}

\section{Identification and phylogenetic analyses of exocyst subunits from Solanaceous plants}

To identify exocyst subunit genes in Solanaceous plants, BLAST analyses were performed by using protein sequences of $A$. thaliana exocyst subunits (listed by Chong et al., 2010) as query against the predicted proteomes of $N$. benthamiana, potato (S. tuberosum) and tomato (S. lycopersicum). Genes for all eight known exocyst subunits were identified in $N$. benthamiana, tomato and potato. Both tomato and potato contain one copy of Sec6, Sec8 and Sec10, two copies of Sec3, Sec5 and Sec15, three copies of Exo84, and multiple copies of Exo70, i.e. 22 in tomato and 21 in potato versus 23 in A. thaliana (Table 1). In contrast, $N$. benthamiana has twice the number of exocyst subunit genes (Table 1) as anticipated based on its allopolyploid genome (Goodin et al., 2008).

TABLE 1. Predicted copy numbers of exocyst subunit genes in Solanaceous plants.

\begin{tabular}{ccccc} 
& A. thaliana & N. benthamiana & Tomato & Potato \\
\hline Sec3 & 2 & 4 & 2 & 2 \\
\hline Sec5 & 2 & 4 & 2 & 2 \\
\hline Sec6 & 1 & 2 & 1 & 1 \\
\hline Sec8 & 1 & 2 & 1 & 1 \\
\hline Sec10 & 2 & 2 & 2 & 1 \\
\hline Sec15 & 2 & 4 & 22 & 2 \\
\hline Exo70 & 23 & 44 & 3 & 21 \\
\hline Exo84 & 3 & 6 & & 3 \\
\hline
\end{tabular}




\section{Chapter 2}

Phylogenetic analysis was performed to reveal the evolutionary relationships among the exocyst subunits from $N$. benthamiana, tomato, potato, and $A$. thaliana. The phylogenetic tree in Figure 1 shows that the $A$. thaliana Exo70 proteins cluster with Solanaceous Exo70s into eight subclades, (i.e. Exo70A-H), which is in line with previous phylogenetic analyses (Synek et al., 2006; Chong et al., 2010; Sekeres et al., 2017). This clustering and the rather comparable number of Exo70 genes in the analysed plant genomes indicate that the Exo70 gene family was already largely present in the last common ancestor of Brassicaceous and Solanaceous plants. There is one branch in the Exo70 tree that only contains Exo70H-genes unique for $A$. thaliana (i.e. Exo70H5, - H6, - $\mathrm{H} 7$, and - H8). Three clusters do not comprise $A$. thaliana orthologues, but solely contain Solanaceous-specific Exo70H subunits (i.e. Exo70H-S1, -S2, and -S3).

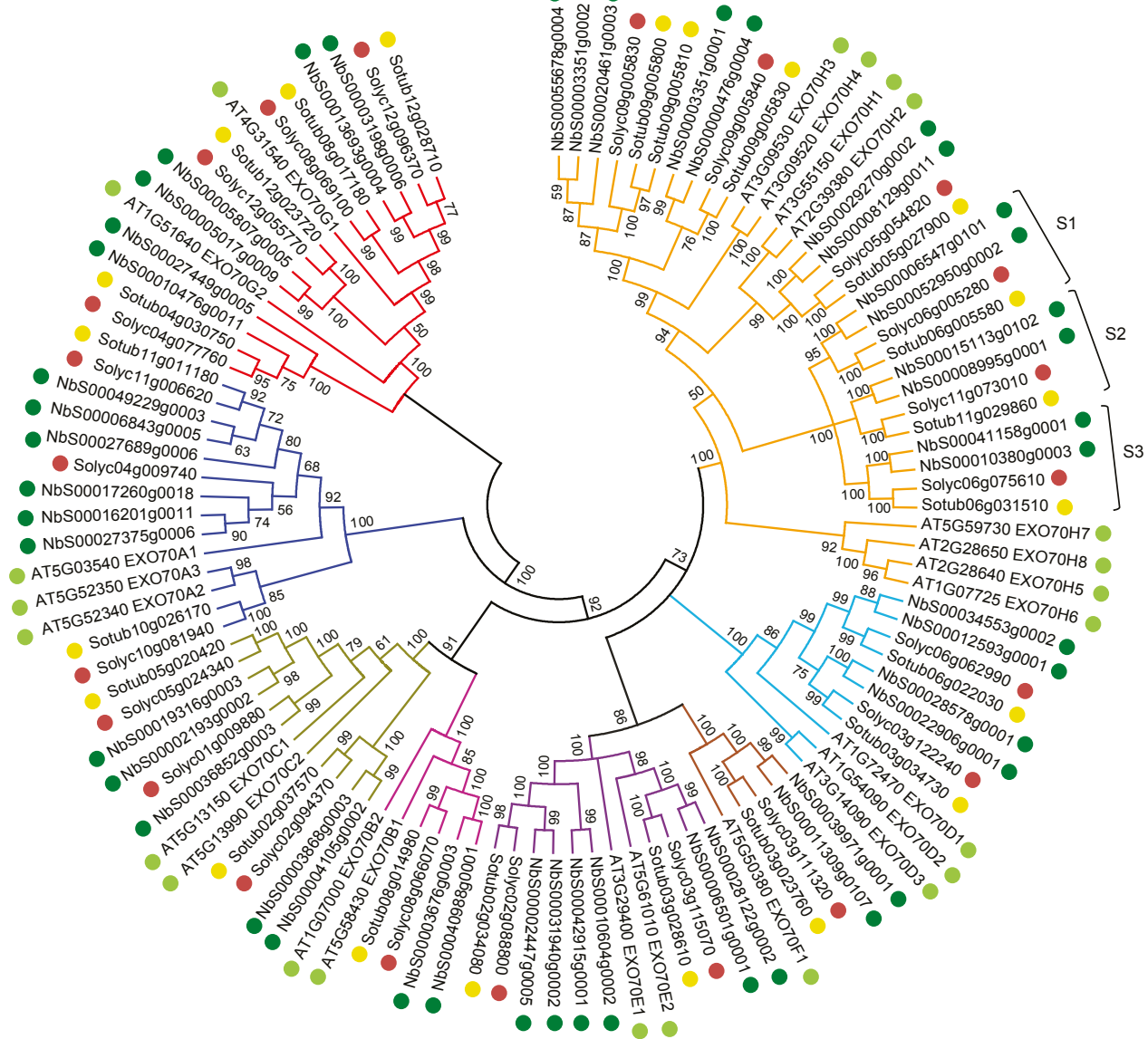

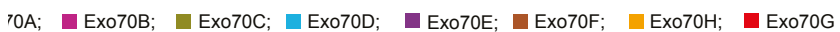

tiana benthamiana; Arabidopsis thaliana; Solanum lycopersicum; Solanum tuberosum

FIGURE 1. Phylogenetic tree based on predicted Exo70 proteins of $A$. thaliana and the Solanaceous plants $\mathbf{N}$. benthamiana, tomato (S. lycopersicum) and potato (S. tuberosum). Exo70 proteins cluster in 
eight subclades distinguishable by the colours of the branches. Exo70H clusters S1, S2, and S3 lack A. thaliana homologues and could be Solanaceous-specific. Protein sequences were aligned with ClustalW at default settings and phylogenetic analysis was performed using the Neighbor Joining method with 5000 bootstraps in MEGA5. Branches corresponding to partitions reproduced in $\leq 50 \%$ bootstrap replicates were collapsed.

The phylogenetic tree of $\mathrm{Sec} 3$ subunits shows that $A$. thaliana $\mathrm{Sec} 3 \mathrm{~A}$ and $\mathrm{Sec} 3 \mathrm{~B}$ group together, whereas the Solanaceous Sec3 subunits are divided into two distinct subclades, with each containing one member of tomato, one of potato, and two of $N$. benthamiana (Figure $2 \mathrm{~A}$ ). Also $A$. thaliana $\operatorname{Sec} 5 \mathrm{~A}$ and $\mathrm{Sec} 5 \mathrm{~B}$ share high sequence similarity and cluster together in a manner comparable with that observed for Sec3 (Figure 2B). As such, it is not possible to distinguish Solanaceous Sec3A from Sec3B, or Sec5A from Sec5B. It appears that duplication of Sec3 and Sec5 occurred twice independently, once in the lineage comprising $A$. thaliana and once in the common ancestor of Solanaceous plants.

In contrast to Sec 3 and Sec5, the two copies of $A$. thaliana Sec15 (Sec15A and Sec15B) are not clustered but instead group with their homologues in Solanaceous plants (Figure 2F). Similarly, the three $A$. thaliana Ex084 paralogues were found to cluster with homologues from Solanaceous plants into three subclades. This indicates that duplication of Sec15 and triplication of Exo84 arose from an ancient duplication in a common ancestor of Brassicaceous and Solanaceous plants (Figure $2 \mathrm{G}$ ). The phylogenetic trees of the singlecopy genes Sec6 and Sec8 show clustering of Solanaceous genes as expected, with the A. thaliana gene on a separate branch. Sec10 was previously reported to be a single copy gene in the A. thaliana reference genome. However, Vukašinović et al., (2014), who resequenced the Sec 10 locus in A. thaliana, revealed that it comprises two paralogous genes in tandem that are almost identical.

Our results are in agreement with the findings reported by Cvrčková et al. (2012), who analysed the evolutionary relationship of exocyst subunits from 10 different plant species, and distinguished three major groups. The first group includes the low copy number gene families Sec3, Sec5, Sec6, Sec8 and Sec10, which are duplicated in one or more plant species probably due to relatively recent gene duplication events that occurred independently in different plant lineages (Cvrckova et al., 2012). The second group comprises the exocyst subunits Sec15 and Exo84. They are encoded by small gene families that emerged from a single ancestral gene. This expansion occurred much earlier during plant evolution than the gene duplications that shaped the first group. Similar to the second group, the third group comprising the multi-copy Exo70 gene family with an enormous diversity among its paralogues, probably evolved from ancient gene duplications in the common ancestor of land plants (Cvrckova et al., 2012). 


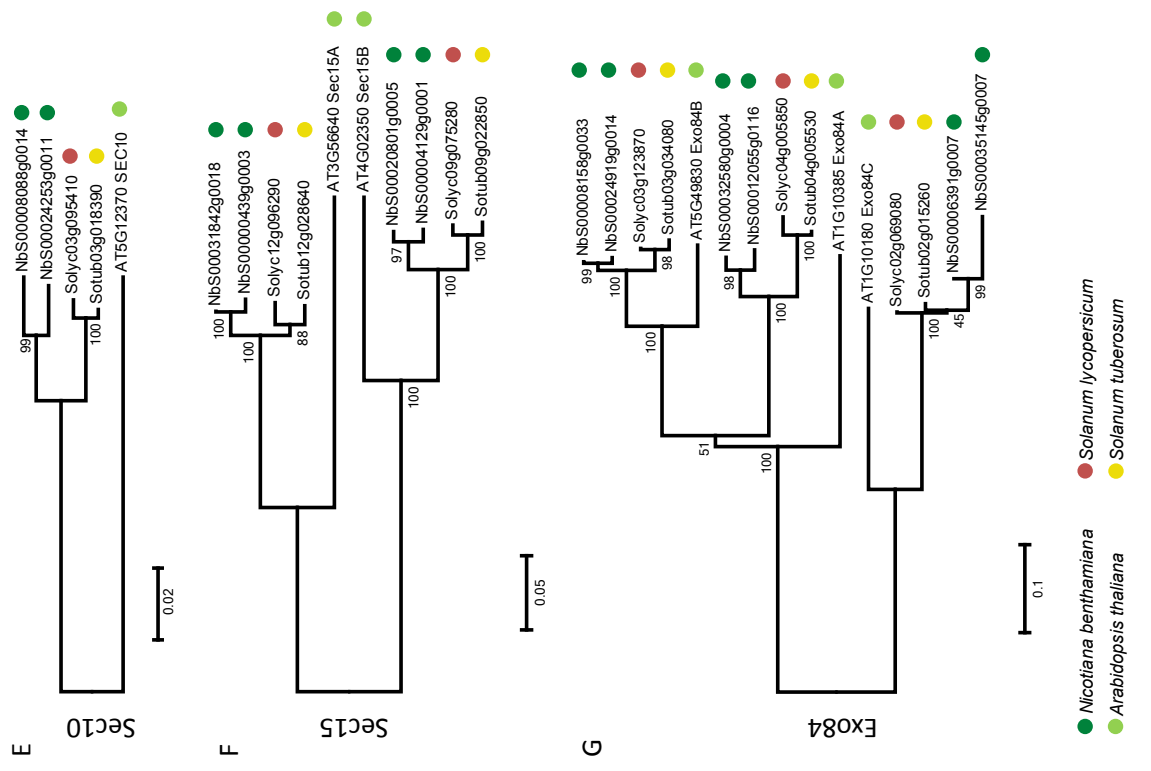

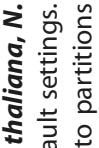

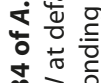

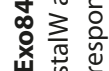

突蒙

है

는

ज 훙

원

๗ 乞ั

过

o jँ

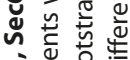

นู่

ฝู ธิ भु.

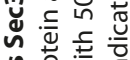

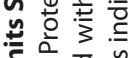

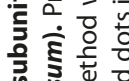

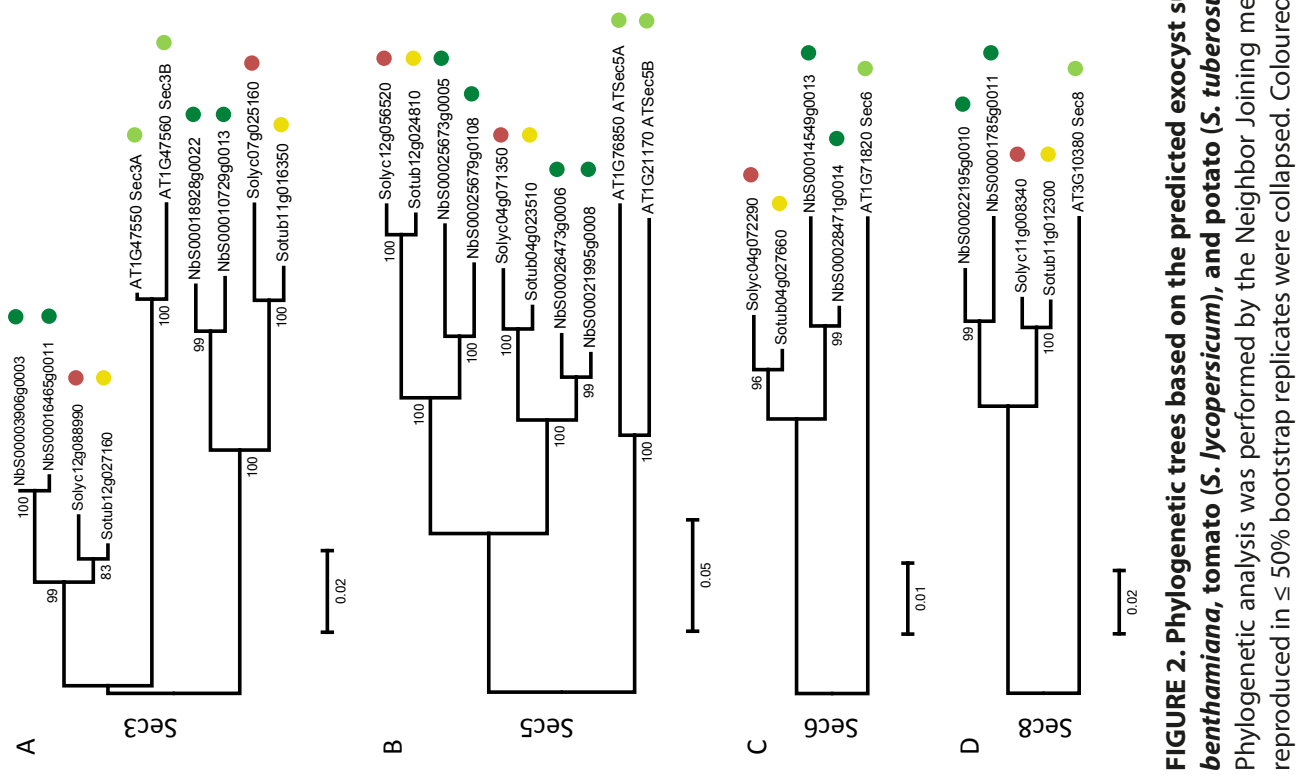




\section{Silencing exocyst subunit genes results in a range of developmental phenotypes}

To study the function of the individual exocyst subunits in plant immunity, we made use of VIGS in N. benthamiana (Ratcliff et al., 2001), preventing lethality issues upon nullmutations (Zhang et al., 2010). We generated VIGS constructs with a binary TRV vector as backbone that have the potential to silence paralogous exocyst subunit genes. For the subunits in the first major group, Sec3, Sec5, Sec6, Sec8, and Sec10, we were able to generate one construct per subunit that targets all paralogues in $N$. benthamiana. For the others, Sec15, Exo70, and Exo84, which have a lower sequence similarity, we needed multiple constructs per subunit to accomplish silencing of all subunit genes (Supplementary Table S2). Three weeks after treatment of $N$. benthamiana with the various exocyst TRV-constructs, transcript levels of the targeted genes were found to be reduced in comparison with the levels in TRV:GUS-treated control plants (Supplementary Figure S1). The silencing levels varied and only three genes, namely EXo70D1, EXO70E1, and ExO $70 H-S 3$, showed a silencing efficiency of $\leq 40 \%$.

Three weeks after TRV-treatment, we monitored changes in plant development. The results show that silencing of the exocyst subunit genes with low copy numbers ( $\mathrm{Sec} 3$, Sec5, Sec6, Sec8, and Sec10) leads to aberrant plant development (Figure 3). The whole plants and also individual leaves were smaller in comparison to the control (TRV:GUS), and in particular Sec10-silenced plants were found to be severely dwarfed. Also silencing of some members of the extended Exo84 and Exo70 gene family, namely Exo84A, Exo84C, Exo70A, Exo70C, Exo70D, and Exo70G, resulted in growth retardation, whereas silencing of the remaining members of these families did not cause any changes in growth morphology. In the case of Sec15, the leaves were larger in comparison with the control, which indicates that Sec15 is somehow involved in negative regulation of plant growth. Functional redundancy might explain the observed phenotypic differences among various Exo70 family members. Dwarfism was also observed in mutant lines of the $A$. thaliana exocyst subunit Sec6, Sec8, Exo70A1, Exo70B1, and Exo84B (Cole et al., 2005; Synek et al., 2006; Fendrych et al., 2010; Kulich et al., 2013; Li et al., 2013; Wu et al., 2013) and the moss Physcomitrella patens subunit gene Exo70.3d, which encodes an Exo70G subunit (Rawat et al., 2017).

\section{Exocyst subunits play a role in plant defence against Phytophthora infestans}

Previously, we showed that Sec5 plays a role in plant defence against the oomycete pathogen $P$. infestans (Du et al., 2015a). To investigate whether other exocyst subunits are required for defence against this hemi-biotrophic pathogen, we first silenced the exocyst subunit genes in $N$. benthamiana and subsequently inoculated the plants with P. infestans isolate 14-3-GFP. Six days after inoculation, plants silenced for Sec5, Sec6, Sec8, Sec10, Sec15A, Exo70B, and Exo84B showed significantly larger lesions compared with the TRV:GUStreated control plants (Figure 4), thus pointing to increased susceptibility to $P$. infestans. In contrast, silencing of Sec3, Sec15B, Exo70A, Exo70C, Exo70D, Exo70E1, Exo70E2, Exo70F, Exo70G, Exo70H, Exo84A, and Exo84C did not cause significant changes in lesion sizes in 
comparison with the control. Apart from Sec3, all low copy number exocyst subunit genes seem to be required for defence against $P$. infestans. This is in contrast to the expanded Exo70 gene family of which only subclade Exo70B seems to be involved in defence against $P$. infestans. In other studies, subclades Exo70F and Exo70H1 were found to be required for penetration resistance against $B$. graminis in barley and $A$. thaliana, respectively (Pecenkova et al., 2011; Ostertag et al., 2013). Moreover, Exo70H1 also plays a role in immunity against the bacterial pathogen P. syringae (Pecenkova et al., 2011). The fact that we found no indications for a role for these subclades in Phytophthora resistance suggests that plants have multiple exocytotic pathways, with each pathway operating in defence against a subset of pathogens or classes of pathogens. As hypothesized by Žárský et al. (2013), these exocytotic pathways might be mediated by different Exo70 sub-complexes.

A
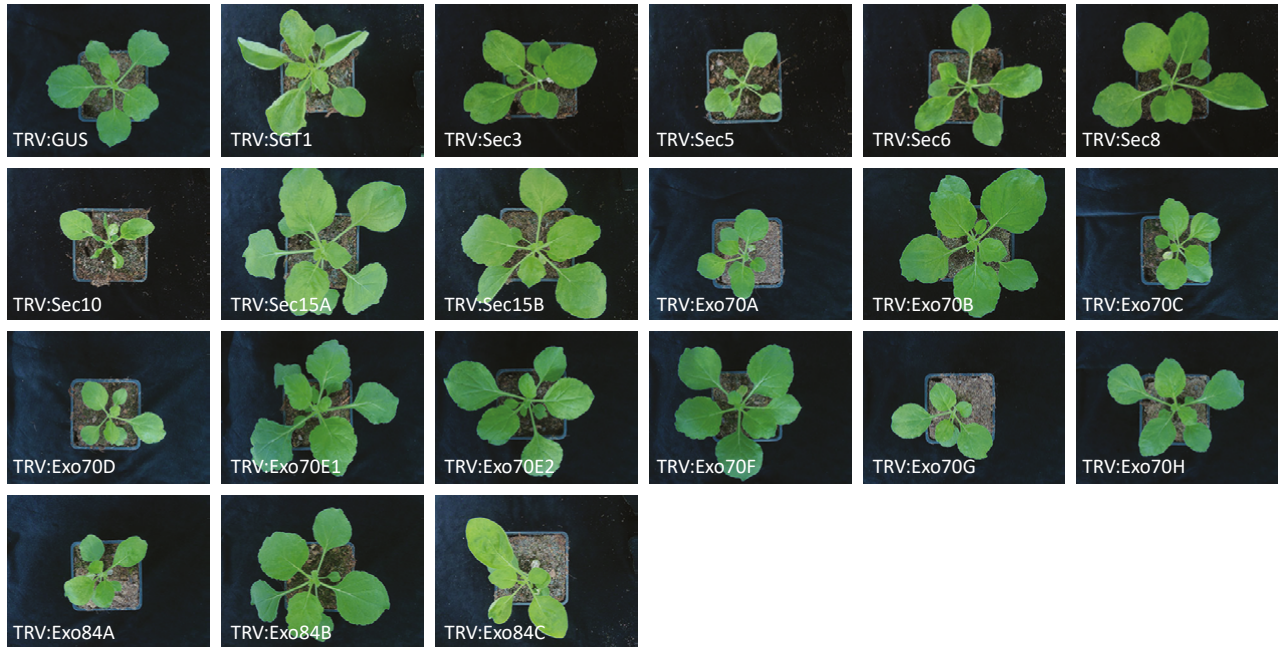

B

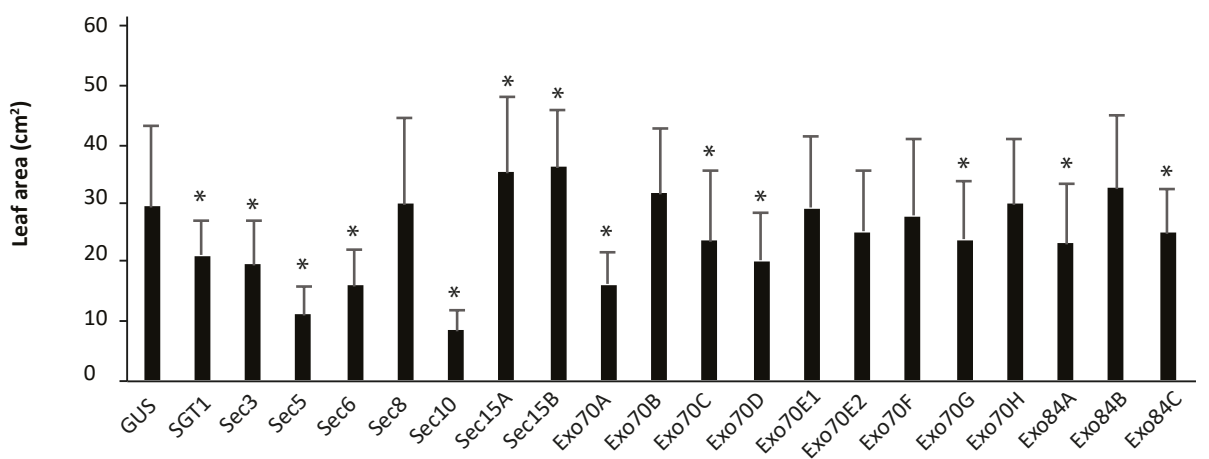

FIGURE 3. Developmental phenotypes. (A) Morphology of $N$. benthamiana plants upon silencing of exocyst subunit genes. Representative pictures were taken 3 weeks after infiltration with the TRV-silencing constructs. Plants infiltrated with TRV:GUS and TRV:SGT1 constructs were included as controls. (B) Surface area of leaves of $N$. benthamiana plants upon silencing of exocyst subunit genes. Surface areas of leaves of similar age from 
3-week old plants were measured and calculated using Image J software. Error bars indicate SDs in at least three independent biological experiments. Significant differences compared with TRV:GUS-treated control plants are indicated by asterisks ( $n \geq 20$ combined from at least three independent experiments, one-sided Student's t-test; $\left.{ }^{*} \mathrm{P}<0.05\right)$.

A

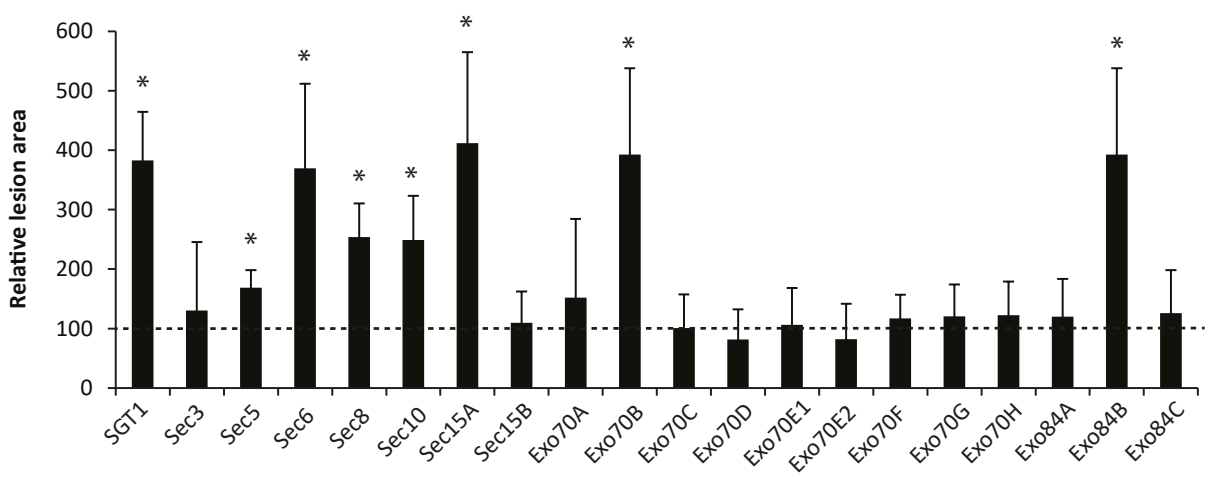

B
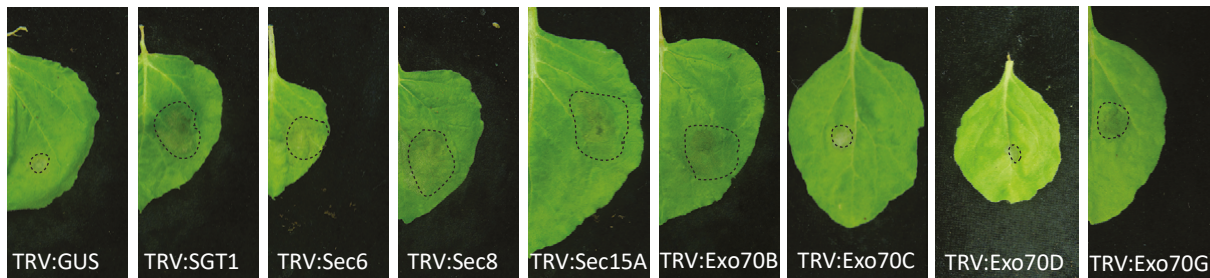

FIGURE 4. The majority of exocyst subunit gene-silenced $\boldsymbol{N}$. benthamiana plants show enhanced susceptibility to $\boldsymbol{P}$. infestans. (A) Lesion diameters were measured at 6 dai with $P$. infestans, and average lesion areas were calculated and normalized to the GUS-control, which is set at 100. Error bars indicate SDs. Significant differences compared with the control are indicated by asterisks ( $n \geq 12$, one-sided Student's t-test; ${ }^{*} P<0.05$ ). Experiments were performed in multiple batches, and repeated at least three times with comparable outcomes. (B) Examples of lesions caused by $P$. infestans isolate 14-3-GFP on $N$. benthamiana leaves silenced for various exocyst subunit genes. Pictures were taken 6 dai.

\section{Exocyst subunits are required for plant defence against the bacterial pathogen Pseudomonas syringae}

We also investigated whether exocyst subunits are required for defence against the biotrophic bacterium Pss. We selected 12 exocyst subunits based on published data (Pecenkova et al., 2011; Stegmann et al., 2012; Kulich et al., 2013; Stegmann et al., 2013; Zhao et al., 2015) and on the phenotypes that we observed upon infection with P. infestans. TRVtreated $N$. benthamiana plants were spray-inoculated with Pss isolate B728a and bacterial growth was checked 3 dai. As shown in Figure 5, plants silenced for Sec5, Sec6, or Sec10 showed a significant increase in bacterial growth compared with the TRV:GUS-treated controle plants, whereas silencing of Sec3, Sec8, Sec15A, Sec15B, Exo70A, Exo70B, Exo70D, ExO70G, or ExO70H did not cause significant differences in bacterial infection. Others 
reported that $A$. thaliana Exo70B and Exo70H mutants showed enhanced susceptibility to Pst (Pecenkova et al., 2011; Stegmann et al., 2012; Stegmann et al., 2013; Zhao et al., 2015), but in our assays we found no indications for a role for N. benthamiana Exo70B and Exo70H in resistance to Pss.

A

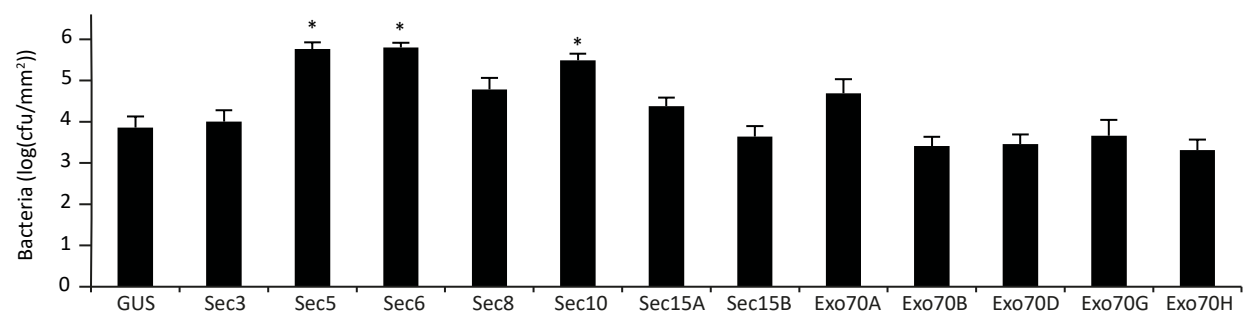

B
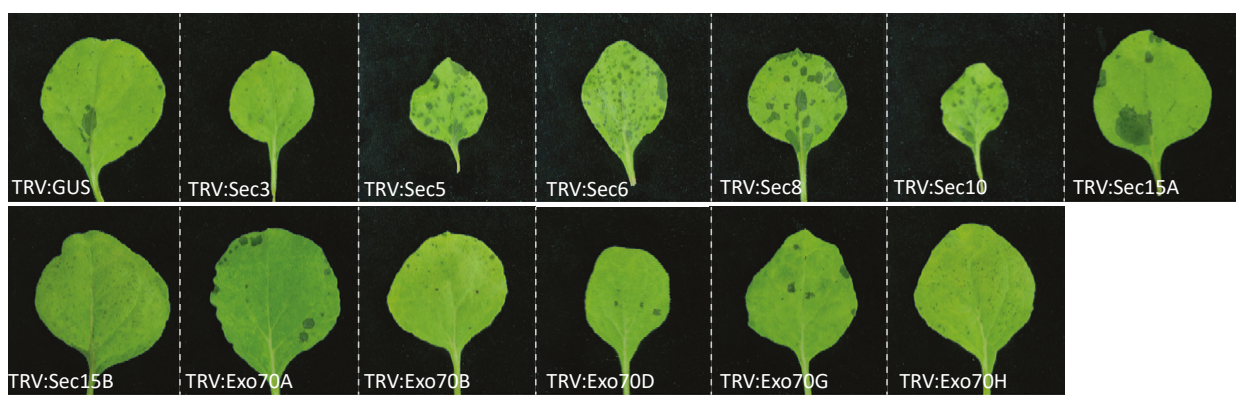

FIGURE 5. Response of exocyst subunit-silenced $\boldsymbol{N}$. benthamiana plants to Pseudomonas syringae. (A) Bacterial growth was measured at 3 dai. Error bars indicate the SDs. Significant differences compared with the control TRV:GUS ( $n=4$, one-sided Student's $t$-test) are indicated by asterisks (* $P<0.05$ ). Experiments were repeated three times with comparable outcomes. (B) Leaf spots caused by Pss isolate B728a on N. benthamiana leaves silenced for various exocyst subunit genes. Pictures were taken 3 dai.

\section{Several exocyst subunits facilitate host susceptibility to the necrotrophic fungus Botrytis cinerea}

To explore the role of the exocyst subunits in defence against necrotrophic pathogens, we performed infection assays with the broad host range fungus $B$. cinerea. TRV-treated $N$. benthamiana plants were inoculated with spores of $B$. cinerea isolate B05.10. As early as 2 dai, two types of necrotic lesions could be discriminated, namely halted primary lesions and expanding secondary lesions (Figure 6A; van Kan, 2006). At 3 dai, $80 \%$ of the inoculated spots on leaves of TRV:GUS-treated control plants developed into secondary lesions, and similar percentages were found on the majority of exocyst-silenced $N$. benthamiana plants. None of the exocyst-silenced plants was found to be more susceptible to $B$. cinerea. SGT1-silenced plants showed fewer secondary lesions and this is in line with the results reported before (El Oirdi \& Bouarab, 2007). In our assays, we observed significantly less secondary lesions on plants silenced for Sec5, Sec6, or Sec10 (Figure 6B). These results are in clear contrast to the enhanced susceptibility that we observed towards biotrophic 
pathogens. This suggests that these exocyst subunits are, on the one hand required for defence against biotrophic pathogens, and on the other hand contribute to colonization by the necrotrophic fungus $B$. cinerea. This apparent dual role of exocyst subunits in defence might be related to the function of the exocyst complex in endomembrane cycling of cell surface receptors. Receptors that recognize PAMPs of $B$. cinerea can induce necrotic responses as shown in the case of the A. thaliana RBPG1 receptor (Zhang et al., 2014), and this is probably favourable for a pathogen that thrives on dead plant cells. On the other hand, receptors that recognize PAMPs of biotrophic pathogens often induce PAMP-triggered immunity (PTI), thereby creating an unfavourable condition for these type of pathogens.

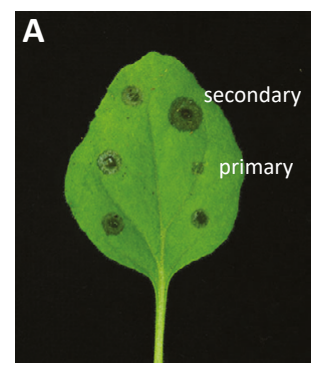

FIGURE 6. Silencing of exocyst subunit genes in $\mathbf{N}$. benthamiana reduces susceptibility to the fungal pathogen $B$. cinerea. (A) Primary and secondary lesions 2 dai with $B$. cinerea isolate B05.10. (B) Percentage of secondary lesions 3 dai with $B$. cinerea. Error bars indicate the SD of at least three biological replicates. Significant differences with the control TRV:GUS ( $n \geq 3$ experiments, one-sided Student's t-test) are indicated by asterisks ( ${ }^{*} \mathrm{P}<0.05$ ). Each experiment contained at least 50 lesions per silenced exocyst subunit.

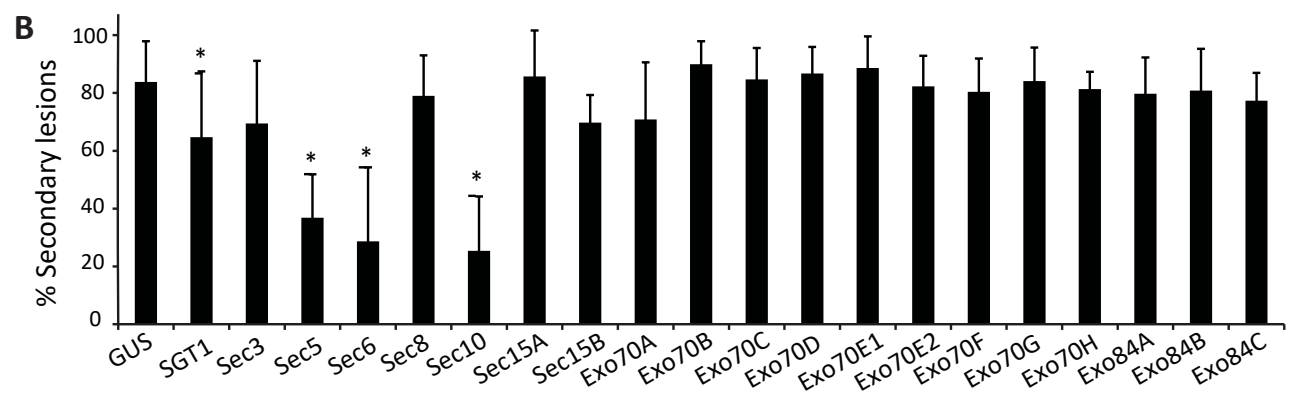

\section{Exocyst subunits are involved in callose deposition}

Callose deposition is a process that probably depends on the exocyst complex. This is exemplified by the fact that Sec5 is required for proper callose deposition during plant defence (Du et al., 2015a). Hence, we wondered whether other exocyst subunits play a role in callose deposition. To test this, leaves of silenced $N$. benthamiana plants were infiltrated with Pst $\Delta$ hrcC to elicit callose deposition. Microscopic analysis of leaves stained with aniline blue revealed that callose deposition in SGT1-silenced plants was significantly reduced compared with TRV:GUS-treated control plants (Du et al., 2015a).

Callose deposition was also significantly reduced in most exocyst subunit gene-silenced plants, with the exception of Exo70A and Exo70D (Figure 7A, B). This suggests that the latter two are not required for callose deposition and this could be explained by the 
diversification of the Exo70 family where specific subunits mediate different exocytotic processes. The reduced callose deposition in most exocyst-silenced plants is in line with the findings by Pečenková et al. (2011) that Exo70B2 of $A$. thaliana is required for proper formation of papillae, of which callose is a major component, and by Kulich et al. (2015) that $A$. thaliana Exo70H4 plays a role in callose deposition in trichomes. Exocyst subunits may have a role in transporting callose synthases to the plasma membrane and this may explain why callose deposition is disturbed in leaves lacking one of the subunits of the exocyst. Notably not all exocyst subunit gene-silenced plants that show reduced callose deposition have aberrant phenotypes in the disease assays (Supplementary Table S4). This could be due to the fact that the pathogen strains that we used are not virulent enough to breach defence barriers other than callose deposition or, alternatively, that the strains are too virulent to see differences in disease phenotypes.
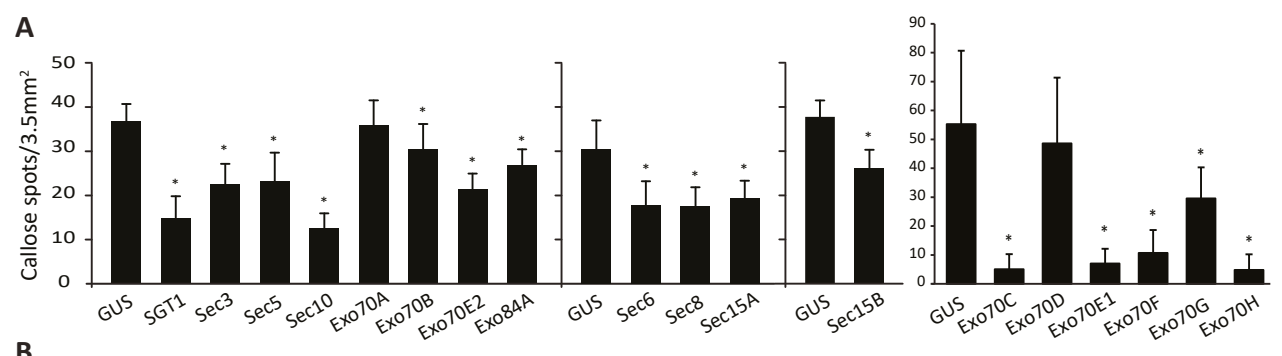

B

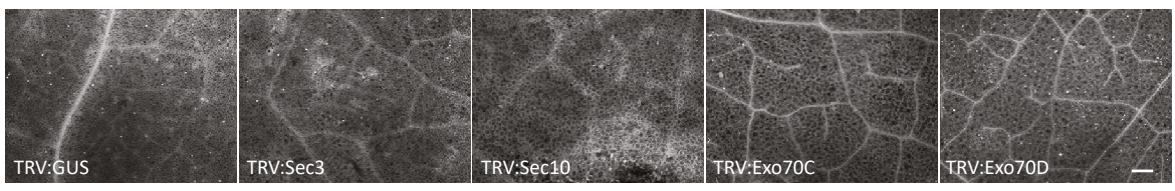

FIGURE 7. Exocyst subunit genes are required for callose deposition. (A) Callose deposition in N. benthamiana leaves $16 \mathrm{~h}$ after infiltration with Pst DC3000 $\Delta$ hrcC. The $y$-axis shows the amount of callose spots per microscopy image (i.e. $3.5 \mathrm{~mm}^{2}$ ). Error bars represent the $\mathrm{SE}$, and asterisks mark significant differences relative to TRV:GUS ( $n \geq 9$, one-sided Student's t-test; $\left.{ }^{*} P<0.05\right)$. Experiments were performed in multiple batches each containing TRV:GUS-treated plants as control. (B) Representative microscopy images showing callose deposition as bright spots upon aniline blue staining. Scale bar represents $200 \mu \mathrm{m}$. 


\section{Conclusion}

In this study we showed that several exocyst subunits function in exocytosis-related processes, including plant development, plant defence and callose deposition. We found a role for three exocyst subunits (i.e. Sec5, Sec6, and Sec10), in defence against all tested pathogens. However, their role changes depending on the type of pathogen that elicits the defence response. They either contribute to defence by raising a barrier against the (hemi-)biotrophic pathogens P. infestans and Pss, or they help the necrotrophic pathogen B. cinerea to colonize. Sec8, Sec15A, Exo70B and Exo84B were only found to function in defence against $P$. infestans. Our study further showed diverse roles for Exo70 and Exo84 paralogues in plant development and defence, which supports the idea that these clades have evolved in such a way that each paralogue has a specialized function, either in exocytosis or in other cellular processes. The present study provides insights into the roles of Solanaceous exocyst subunits in defence against three unrelated pathogens. It forms a platform for further functional studies, in particular on the role of Solanaceous exocyst subunits in immunity and autophagy, as well as the role of salicylic acid and other defence-related hormones in exocyst-mediated defence responses.

\section{Acknowledgements}

Bert Essenstam and Henk Smit at Unifarm are acknowledged for excellent plant care and Jan van Kan for advice on Botrytis disease assays. This research was supported by the Earth and Life Sciences Division and the Technology Foundation of The Netherlands Organization for Scientific Research (NWO; PhD grant to EO and a VENI grant KB, respectively), by the China Scholarship Council (CSC; fellowship to YD), the 111-project 'Crop Breeding for Disease Resistance and Genetic Improvement' (Northwest A\&F University), and by the 'Food-for-Thought campaign' of the University Fund Wageningen. 


\section{Supplemental data}

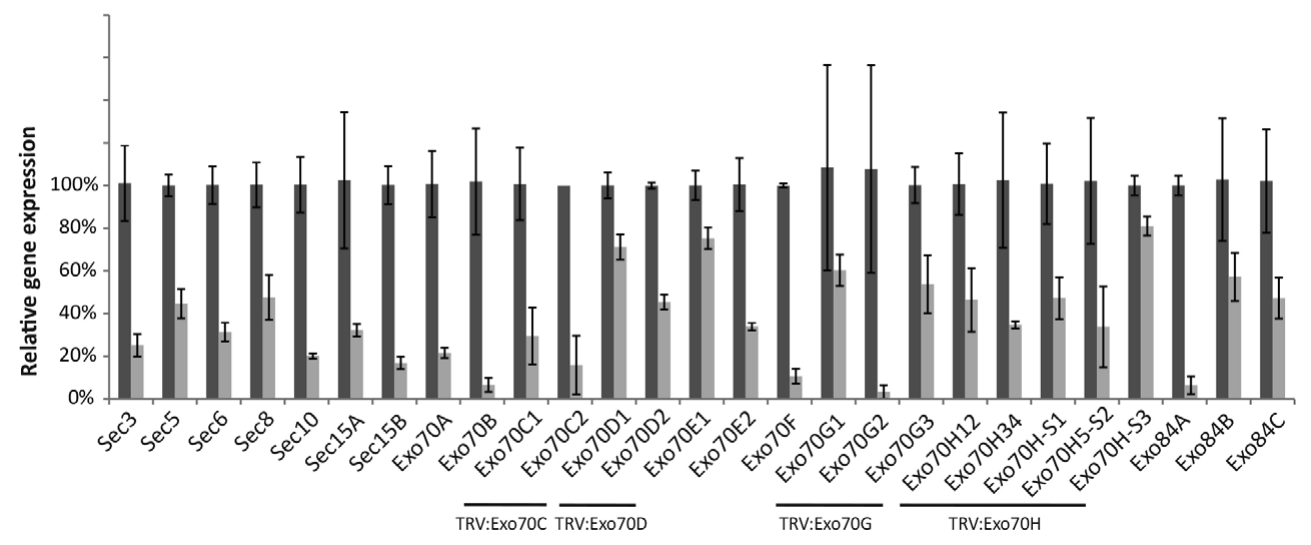

FIGURE S1. Relative expression of exocyst subunit genes in silenced $\boldsymbol{N}$. benthamiana plants three weeks after TRV treatment. Transcript levels were determined by qRT-PCR and normalized to Actin. The bars show the mean fold changes in transcript levels ( \pm standard deviation) relative to the transcript levels in TRV:GUS-treated control plants, which were set at 100\%. ( $\square$, TRV:GUS; $\varpi$, TRV:exocyst subunit). Four of the silencing constructs target multiple genes in one (sub)clade (indicated by the horizontal bars) but expression of each gene in those (sub)clades is monitored separately by using gene-specific primers in the qRT-PCR.

SUPPLEMENTARYTABLE S1. Gene IDs and protein sequences of exocyst subunits of Nicotiana benthamiana, tomato (Solanum lycopersicum) and potato (Solanum tuberosum). This table can be found at JXB online. 
SUPPLEMENTARY TABLE S2. The TRV-silencing constructs used in this study and the sequences of the fragments inserted in the binary vector PTRV2.

\begin{tabular}{|c|c|}
\hline Construct & Insert \\
\hline \multirow{4}{*}{ TRV:Sec3 } & GAATTCCTTCAAAACATTGAGGCATGTGAGTGGTTAACTAATGCTTTACGTGGTCTTGAAGCGCCTAATTTGGATCCTA \\
\hline & GCTATGCAAACATGAGAGCTGTAAAGGAGAAACGATCTGAGCTTGATAAGTTAAAAACAACATTTGTTCGAAGAGCA \\
\hline & TCTGAGTTCTTGAGAAACTATTTTACCAGTTTGGTGGATTTTATGATAAGTGACAAGAGTTATTTTTCTCAGCGTGGAC \\
\hline & AATTGAAGAGGCCTGACCATGCTGATCTGAGATACAAGTGCAGGACATATGCTCGTCTTCTGCAGCATTTGAAGAGTC \\
\hline \multirow{8}{*}{ TRV:Sec5 } & TTGCTAATCGTGCTTTTGGGCCACTATTTGAAAGACAGGCTCAAGCTGAGAAGATAAGATCTGTTCAAGGAATGCTGC \\
\hline & AGAGGTTCCGAACACTATTTAACTTGCCCAGCACGATTCGTGAGAGCATTAGTACTGGTGAATATGACCTAGCAGTCA \\
\hline & GGGAGTACAGGAAAGCGAAGTCAATCGTTCTGCCTTCTCATGTTGGAATTTTGAAGCGCGTGCTTGAGGAGGTTGAA \\
\hline & GAGTTATGCAAGAATTTAAAAGCAAGCTTTATAAGTCTCTGGAAGATCCTCAAATTGACCTAACAAATCTTGAAAATA \\
\hline & ATGTGAGACTCTTGTTGGAGTTGGAACCCGAATCAGATCCTGTGTGGCACTATTTGAATATACAGAACCAGAGAATCC \\
\hline & GAGGTTTGCTTGAGAAGTGCACTTTGGATCATGAAGCAAGGACGGAAAATTTCAGGAATGAGATGCGTGAAAGAGC \\
\hline & TTTATCTGATGCAAAATGGAGGCAAATTCAGCAAGATTTGAACCATTCTTCAGATGTTGATTACTCTGATTCTCATGAA \\
\hline & AATACATACTTGCCGGGTGATTCACAACAAGT \\
\hline \multirow{5}{*}{ TRV:Sec6 } & TTGACAAGAATTGCTTGGGCCCTTTAAGAAAAGCTTATTGCACATCCCTTAATTTGCTTTTTACGCCGGGAGCTCTGGA \\
\hline & GCTAAGTTCTAGTACTCTTGAAGCTCTCCCACAGAACTATGCCGAGCAGGTTAATTTCGAGGACACATGCAAAGGTTT \\
\hline & TTTAGAGGTTGCAAAGGAAGCTGTTCATCAAACTGTCAGTGTCATCTTTGAAGATCCAGGAGTAGAGGAATTGCTCGT \\
\hline & TAAGTTGTATCAGAAAGATTGGTTGGAAGGACAGGTTACCGAGTTTTTAGTTGCAACATTTTCAGACTACTTCACCGA \\
\hline & TGTTAAAATGTACATTGAAGAAAGATCATTTAGGCGTTTTGTCGAGGCTTGCCTGGAGGAGACTGTTGT \\
\hline
\end{tabular}
CTACAGACATCAGTTGGAACCCTGATTCAGATGCATCTCGTGACACTGGAGGCTATAGTATTGGCTTTTCATTGACCGT CTTACAGAGTGAATGCCAACAACTCATATGTGAGATCCTGCGAGCTACTCCAGAAGCTGCATCCGCAGATGCTGCTG

GAATTCGCACCGCAAGACACTTTCTGAGCTGGAAAAAGGTGTAGACGGCTTGTTTGATAGCTTTGCACGGTTGGATT TGCGTATATCTAGTGTGGGTCAAACAGCTGCCAAAATAGGAGATCATCTGCAGAGTGCAGATGCTCAGAGAGAAGTTG

TRV:Sec10 CTAGTCAGACAATAGAGCTCATAAAGTACTTGATGGAGTTCAATAGCAGTCCAGGAGACCTGATGGAACTTTCTCCAT TGTTTTCTGATGATAGTCGTGTTGCTGAAGCTGCTTCGATTGCTCAAAAATTAAGATCATTTGCTGAGGACGATATTGG AAGGGATCC

AGATCTAGGTCCTATGGTCAGGCTTTCTTTTGAAAGCGGGAAGCCTGATGCCCTTCTACAACAGCTTAAAAATGTCGT CAAAAAGAAAGAAGTTGAAATAGAGGAGCTTTGTAAGCTTCACTATGAGGAATTTATTGTTGCAGTGGATGAGCTGC

TRV:Sec15A GCGGTGTCTTGGTTGATGCAGAAGAGCTAAAAGCTGAGTTGCAAACTGATAATCTGAAGCTGCAAGATGTTGGGAGT ACCCTTCTTTTAAAACTTGAAGAGTTTCTTGAATCTTATTCAATTAAGAAAAATGTTACAGAAGCGATTAAGATGTCAG GCAACTGTGTCCAAGT

TGCCTGCATTTCCTTATGTTGCACCGTTTTCGTGTACGGTGCCTGATTGCTGTAGAATAGTGCGGTCCTTTATCGAGGAT

TRV:Sec15B TCAGTAAGTTTTATGTCACATGGTGGTCAGCTTGATTTCTATGATGTGGTGAAGAAGTACTTGGATCGGCTTTTAACTG AAGTCCTGGATGGAGCCCTGTTGAAGCTCATTCATACGTCTATCGGTGGTGTTACCCAAGCAATGCAGATGGCAGCTA ACATGGCTGTGTTTGAACGCGCTTGTGATTTTTTCTTTCGTCATGCTGCACAGCTTTCAGGGATTCCATTGA ACAGGCAATCCCGTCCTATAGAGCAGAGAGAACTGAAACGGAGACTCCAACGTTTAGTTGATCAGCTCCGTGACAGC

TRV:Ex084A TTTTGTAGGCAACATGCACTTGAACTCATTTTCTTGGAAGATGGTGGCGTTCGTTTAAGTCCAGACATGTACCTAAATA TGGAAGGAAGTCCAGAAGAGATAGAGTGGTTTCCATCTCCAGTATATCAGGAAATGTTTGAGAAGTTGACTCGAATTG CTAGCATAGCATCAGACATGTTTGTGGGAAGAGAAAGATTTGCTACTATTCTTTTGATGAGACTCACTGAAACTATCA AGGCTGGCGATTAGCGAACCAACAACACAATCAAACAAATTTTTATTCTCTTTCTACGAACAACTAATAATCTCCTCTT CTTTCGTCAATCATGGCGATGGCGATAACAACTCTCACCTTAGGATTAGATCTACCCTTTTGATTTTCTTACGTCTTCCCT TRV:Exo84B ATTTTTGCTCTATATTTCAACCCTAAACATCCGCCATTAAGATTCACCTCATGGCTTCCGTCAAATCCTCTCGTTCAAGA GCTCATGCGGTCACTCAGTCTAAAGGAATCAACAAGGATACCGGCCCCAAGCTCGAAGAAAATCTTAACGTCTTCAA ATCCGACAATTTCGATGCTGATGCTTTTGTCCAGTCCAAATGTCATTCTCTCAACGAGAAGGAAATAAGGCAGCTGTG CTCTTATCTATTGGAACTGAAAAGGGCTTCTGCTGA 
SUPPLEMENTARY TABLE S2. (Continued)

\begin{tabular}{|c|c|}
\hline on & \\
\hline TRV:Ex084C & $\begin{array}{l}\text { GAATTCTAGTCAAAGAAATAGTGGAGAAGCTCACCCAGTTAGTCATTCTACATTTTGGAGCTAACATATTGACCAGAAT } \\
\text { TTCACATCTTTTTGACAAGTATGTGGATTCTTTGATTAAAGCCCTGCCTGGTCTTTCTGAGGATGATAATCTCACTGAGC } \\
\text { TGAAAGAACCTGTTCTTTTTAGAGCTGAAACAGACTCGGAGCAGCTTGCTCTATTGGGAACTGCATTTACCATTGCTG } \\
\text { AGGAACTATTGCCTATGGTTGTATCTAGAATTTGGAATGTTCTTAATGAAAGCAAGGAAGCGAGCTC }\end{array}$ \\
\hline OA & $\begin{array}{l}\text { ATTCATTTTATGCGGATTGCTGTCAAATTGTTATTTGCTGCGGAGCGAAAAGTTTGTGATCAAATGTTTGAAGGATTTG } \\
\text { AGCACCTCAAAGATCAATGTTTTGCTGAAGTTACTACTGGGAGTGTGGCTGTGCTGCTTAGTTTTGGTGATGCAATTG } \\
\text { CAAAAAGCAAAAGATCACCTGAGAAGTTGTTTGTGCTTCTAGATATGTATGAAATAATGCGGGAACTTCATTCAGAGA } \\
\text { TTGAATCGCTTTTCATAGGTAAAGCTTGCAATGAAATTAGGGAATCTGCCTTTGGTTTGACA }\end{array}$ \\
\hline B & $\begin{array}{l}\text { TGTTTATGAGGCGCTTAGGGATTTGATGCCTGAATTTGAATTGTTGTTTATGGATCAATACTGTGTGTTATTGAGGAATG } \\
\text { AAGCTTTGACTATATGGAGAAGATTAGGTGAGGCTATTAGAGGGATTTTTATGGATTTGGAGAATTTGATTCGCCGTGA } \\
\text { TCCTGCCAAAACGCCTGTTCCAGGTGGTGGACTCCACCCGATTACTCGATACGTGATGAATTATCTCCGCGCAGCTTGT } \\
\text { CGATCCCGGATTTCTCTTGAACAGGTTTTTGAGGAGTCTGCTAGTGTTGTTGATTATAGAGGAGAAGTGGATGATAGA } \\
\text { GCGCTTTCATCGTCTCCCTTGGCTGTTCAAATGGCGTGGACCATGGAGTTACTTGAGAGTAATCTGGA }\end{array}$ \\
\hline $\begin{array}{l}\text { o70C } \\
\text { s C1 } \\
\text { ) }\end{array}$ & $\begin{array}{l}\text { AATTCGAGTACAAATATGCTTATTCAATCAGCCGCGTTGATGGTGTTCTTCAACGCGCAATGTCCTGAGAAAATCTTCA } \\
\text { GCAATTTGTCTCGAGGTATGATGATCCAACTCCTTAATTTCGCTGAAGCCGTTTCAAGTGATGGGCGATACGTGGTGTA } \\
\text { GGAGACGATCAACGATCGTTAGACATTACCACAAGAATTACCAAAGGGAAACATGGGGTAAGGTGCTCCAAATCAAT } \\
\text { GGGAAAGTGACAAAGCCAGTAGTGAAAGAGAGGTTCAAGAATTTCAGCACAATGCTCGACGAAATCCATCGTACGC } \\
\text { AGAGCACTTGGGTGAGCTC }\end{array}$ \\
\hline $\begin{array}{l}\text { RV:Exo70D } \\
\text { argets D1 } \\
\text { nd D2) }\end{array}$ & $\begin{array}{l}\text { GAATTCCAGTTATCGATCTACTAACAGCATTCGTGAAGTCGATCTCATGCCGTCAGAAGCAATATACGATCTCCGGTGCA } \\
\text { TCGCGGAGCGAATGATTTCAGCTGGATATCTCCGGGAGTGTATTCAGGTGTATGGCAGTGTACGCAAGTCTGCTGTGG } \\
\text { ATTCGAGCTTCCGGAAGCTTGGAATAGAGAAGCTGAGCATAGGAGATATACAGAGACTTGACTGGGAGACTCTCGAA } \\
\text { GCAAAGATTCGTAGATGGATACGAGCAGCAAAAGTATGCCTTCGCATACTTTTCGCTAGCGAGAAGAAGCTCTGTGAG } \\
\text { CAAATGAGCTC }\end{array}$ \\
\hline
\end{tabular}

GAATTCTGATGAACAACATCCATTACATGGTTCAGAAAATCAAGAACTCCAAAATTAGAACTTATTTTGGTGATGAGTG

TRV:Exo70E1 GATAAAAACACATATTGTAAAATACGTGCAGCACGAAAAAAGCTACGAGAGAATAACATGGAGTTCAATCATATCTTTG ATAACCGGTTATGAGAAGTTAGGGAAGGCAGTTCTGAAGGAGAGGTGCAGAAATTTCAGTATTGCTTTTGAGGAAGT GTATAAAAACCAGACAGGATGGACTATTCCAGATATTGAGAGCTC

GCATTTATCAATGTCAGAAAAGATGCCTTGGACGATTGCCTCTTCATTCTTGAAGTTGAGAGGTTGAGCATTGAGGAT GTACTGAAGATGGAATGGAACTCGTTGAACTCCAAAATCAGGAGGTGGATACGGGCTATGAAGATCTTTGTGCGCGT

TRV:Exo70E2 TTATCTTGCCAGTGAAAAGTGGCTAAGTGATCAGATTTTTAGCGAGCTGGAAGCAGTTAGTTCAGTTTGCTTTGCTGA GGCCAAAAGCTTCAATCGTACAGCTTCTGAACTTTGGTGAAGCCATAGCTATTGGCCCTCATCAACCGGAGAAGTTG ATTCGGATTCTTGACATGTATGAGCTGCTCGCAGATCTTATCCCAGATATTGATGCTATGTACTCTGA

GAATTCATTGATCTGAGAGAGATTGCTAACCGTATGATTCGATCTGGATATGAAAAGGAATGTTGTCAAGTGTACTCTA GTGTTCGTAGAGAGGTGCTTGATGAGTGTTTAGCAATTCTTGGCATTGAGAAACTGAGCATTGAGGAGGTCCACAG

TRV:Exo70F GATTGAATGGCAGTCGCTGGACGAGAAAATGAAGAAATGGATTTATGCTGTAAAAGTTCTGGTGAGAATCCTTTTAT CTGCTGAAAAGAGCTTGTGTGAACAGATTTTTGTAGGTTCTGAGTTGATTAAAGAAGTGTGCTTTATGGAAACTGCA AAGGGCTGTGTGAGCTC

TRV:Exo70G GAATTCGACAAGATAATGGCTGTCTTCTTTAGATTCGGGGAAGGGGTTGCACGAAGTAAGAAAGAACCCCAAAAGCT (targets GTTCAAGCTGTTAGATATGTTTGAATCATTGGAAAGGCTAAAATGGTAGTGTTCCGAGGCTGGTTAACTTTGTCAGTGA G1-3) GTATTGTAATCAGATTCTTGAGGATGAATATTGGTCCACACTTATCCAAGTTGTGGAGATACATCAAAGTTTGCAGGCA CTTAATTTGGATTACCTTGAGATTTCAGTCTCCGAGTTCAATGACGTGCAGAGCATAGAGGGTCACATTGCTCAGTGG GGTAAGCATTTAGAATTTGCTGTAAAGAGCTC

TRV:Exo70H (targets H1-4 and S1-3)
GAATTCAAACTTGATGCAAAAGGCTATGAAAAGACTTGAAAATGAATTTTACCAAATTTTGTCAACAAACAAGGAGC ATTTAGATCCAGAATCAGTATCTAAGTCTAGTCGTTAGGTGAAAATTGGATATCAAAACAGGAGGGAAAAATCAAGCA GTTTGCATCAAACTATGAACGGCTAGGATGGAGCCACGTCATTGAATCTCCCTTTTATGTAAACTCGATGGTAAAGCA CAACTTTACAAGGACGTTTCATTATCCTATCTGTTCTTAGCGAACAACTTAAACTATGTCGTTTCAGAGCTC 
SUPPLEMENTARY TABLE S3. Primers used for qRT-PCR.

\begin{tabular}{|c|c|c|c|}
\hline Primer name & Sequence $\left(5^{\prime}-3^{\prime}\right)$ & Primer name & Sequence $\left(5^{\prime}-3^{\prime}\right)$ \\
\hline Sec3-rtF & AGTTTGCAAACGAGCTCCGTG & Exo70D1-rtF & TCCCAGCTTATAGATCATTTCTC \\
\hline Sec3-rtR & ACTCTCATCCACAAGTAGAGG & Exo70D1-rtR & CTGAGATCTTCTTCTTATGTGTTGTG \\
\hline Sec5-rtF & AGTTAAGACGGATTGAGGAAGACC & Exo70D2-rtF & CATGAACTATATAAGTTTAATCTCGG \\
\hline Sec5-rtR & TAGTGGCCCAAAAGCACGATTAGC & Exo70D2-rtR & GACAATAATCCAAATCAAATGAAGC \\
\hline Sec6-rtF & TCATGAATGCGTATGTTGAGAGAATG & Exo70E1-rtF & GGCCAAGCTGTAGCTATTGG \\
\hline Sec6-rtR & CTCGTACAATTTGCACCTGCT & Exo70E1-rtR & TGCAGAATCTCCAAGATTTTTCA \\
\hline Sec8-rtF & ATATACCGGCCTGTTCTTCAG & Exo70E2-rtF & AACAGGCAGCCTTTTGAGC \\
\hline Sec8-rtR & TGTACAGCTTTCCGATAATCAAC & Exo70E2-rtR & AACATCAAATTGGCAATGCATC \\
\hline Sec10-rtF & ACACCACCAGATGCAGGAA & Exo70F-rtF & ATGCAGCTCTTGAATTTTGGAG \\
\hline Sec10-rtR & TCTTGGACAGCAACTTCCTTC & Exo70F-rtR & TTCTCCTAGGCCATCTAACAC \\
\hline Sec15A-rtF & TGCTATCAAGGCTATTGATCTG & Exo70G1-rtF & ACCAAAGTCTTCTTTGAGCT \\
\hline Sec15A-rtR & AGTTTGCCCAATATCCTTTGC & Exo70G1-rtR & TCCAGCTTTCCATATTTGTTC \\
\hline Sec15B-rtF & TGAAGAGATGCTTTCAGGTCTG & Exo70G2-rtF & AACCATTGGTACTTGTGCAAG \\
\hline Sec15B-rtR & TCTCTTCAGAACTTGAACAGG & Exo70G2-rtR & TTAACTACTTGTCGATCAATGGC \\
\hline Exo84A-rtF & TCTGAAGATCAAAATTTCTGGGAAG & Exo70G3-rtF & GAAACGATTAAAAGCTTTTAATG \\
\hline Exo84A-rtR & AGCAACTGCTTCAATAGCTCTGC & Exo70G3-rtR & TCCTTCTTGTTCAACCAAC \\
\hline Exo84B-rtF & TGCTCCTTAATGCACATTACCAG & Exo70H12-rtF & AACTATATGCAACAAACTATG \\
\hline Exo84B-rtR & TGTATAAGCTGGCTCTTTACC & Exo70H12-rtR & ACTTTGATCTCGTCTCGTAG \\
\hline Exo84C-rtF & AGAATCAGCTGGTTGAGATC & Exo70H34-rtF & ACATCATTCTACTTGCTGATTAC \\
\hline Exo84C-rtR & AGTCTCGGGATAACAAGGAC & Exo70H34-rtR & AGTTTGCAGAGAAGAATGAGAATC \\
\hline Exo70A-rtF & AGCAGTTGAGAAGGATGCAAC & Exo70H-S1-rtF & TAACTCGATACGTCATGAACTACC \\
\hline Exo70A-rtR & AGCTGTGAATTTGAATCTCCTCC & Exo70H-S1-rtR & ACAACAGAAGGCGGAGAGTCC \\
\hline Exo70B-rtF & TGATGAATAATGAAAGATACATTGTTC & Exo70H-S2-rtF & TGACTCGGTACGTTATGAATTATCTCG \\
\hline Exo70B-rtR & ACTTAAGCTTTTCTTTCAAAGACC & Exo70H-S2-rtR & CTAGCCACGCAAGGCGGAC \\
\hline Exo70C1-rtF & TGATGAACAACGGGCGATAC & Exo70H-S3-rtF & TAACTCGCTACGTCATGAACTACCTT \\
\hline Exo70C1-rtR & ATTGCAACAGTTTACCCCAAG & Exo70H-S3-rtR & GATAAGCCAAGCGAGCCGTAC \\
\hline Exo70C2-rtF & TTTGAAACAGAATGTTGTCAAGT & Actin-rtF & TATGGAAACATTGTGCTCAGTGG \\
\hline Exo70C2-rtR & GTCTCTTTTCGCCAGGGAACAGAGT & Actin-rtR & CCAGATTCGTCATACTCTGCC \\
\hline
\end{tabular}


SUPPLEMENTARY TABLE S4. Summary of phenotypic characteristics of Nicotiana benthamiana plants in which the various exocyst subunit genes are silenced.

\begin{tabular}{|c|c|c|c|c|c|c|}
\hline Exocyst subunit & Level of silencing ${ }^{a}$ & Leaf size & P. infestans & Pss & B. cinerea & $\begin{array}{c}\text { Callose } \\
\text { deposition }\end{array}$ \\
\hline Sec3 & $<30$ & smaller & $\mathrm{N}$ & $\mathrm{N}$ & N & L \\
\hline Sec5 & $30-50$ & smaller & $S$ & $S$ & $\mathrm{R}$ & L \\
\hline Sec6 & $30-50$ & smaller & $s$ & $s$ & R & L \\
\hline Sec8 & $30-50$ & $\mathrm{~N}$ & $\mathrm{~s}$ & $\mathrm{~N}$ & $\mathrm{~N}$ & L \\
\hline Sec10 & $<30$ & smaller & $\mathrm{s}$ & 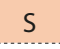 & $R$ & L \\
\hline Sec15A & $30-50$ & larger & $\mathrm{s}$ & $\mathrm{N}$ & $\mathrm{N}$ & L \\
\hline Sec15B & $<30$ & larger & $\mathrm{N}$ & $\mathrm{N}$ & $\mathrm{N}$ & L \\
\hline Exo70A & $<30$ & smaller & $\mathrm{N}$ & $\mathrm{N}$ & $\mathrm{N}$ & $\mathrm{N}$ \\
\hline Exo70B & $<30$ & $\mathrm{~N}$ & s & $\mathrm{N}$ & $\mathrm{N}$ & L \\
\hline Exo70C & $\begin{array}{l}C 1:<30 \\
C 2:<30\end{array}$ & smaller & $\mathrm{N}$ & nd & $\mathrm{N}$ & L \\
\hline Exo70D & $\begin{array}{c}\text { D1: }<90 \\
\text { D2: } 30-50\end{array}$ & smaller & $\mathrm{N}$ & $\mathrm{N}$ & $\mathrm{N}$ & $\mathrm{N}$ \\
\hline Exo70E1 & $<90$ & $\mathrm{~N}$ & $\mathrm{~N}$ & nd & $\mathrm{N}$ & L \\
\hline Exo70E2 & $30-50$ & $\mathrm{~N}$ & $\mathrm{~N}$ & nd & $\mathrm{N}$ & $L$ \\
\hline Exo70F & $<30$ & $\mathrm{~N}$ & $\mathrm{~N}$ & nd & $\mathrm{N}$ & L \\
\hline Exo70G & $\begin{array}{c}\text { G1: } 50-60 \\
\text { G2: }<30 \\
\text { G3: } 50-60\end{array}$ & smaller & $\mathrm{N}$ & $\mathrm{N}$ & $\mathrm{N}$ & L \\
\hline Exo70H & $\begin{array}{l}\text { H12: } 30-50 \\
\text { H34: } 30-50 \\
\text { HS1: } 30-50 \\
\text { HS2: } 30-50 \\
\text { HS3: }<90\end{array}$ & $\mathrm{~N}$ & $\mathrm{~N}$ & $\mathrm{~N}$ & $\mathrm{~N}$ & L \\
\hline Exo84A & $<30$ & smaller & $\mathrm{N}$ & nd & $\mathrm{N}$ & L \\
\hline Exo84B & $50-60$ & $\mathrm{~N}$ & $S$ & nd & $\mathrm{N}$ & nd \\
\hline Exo84C & $30-50$ & smaller & $\mathrm{N}$ & nd & $\mathrm{N}$ & nd \\
\hline
\end{tabular}

a percentage of the expression level in control plants

$\mathrm{S}=$ more susceptible than control plants

$\mathrm{R}=$ more resistant than control plants

$\mathrm{N}=$ no significant difference with control plants

$\mathrm{L}=$ less callose deposition than in control plants

$\mathrm{nd}=$ not determined 


\title{
Chapter 3
}

Interaction between the moss Physcomitrella patens and Phytophthora: a novel pathosystem for live-cell imaging of subcellular defence

\author{
Elysa J.R. Overdijk \\ Jeroen de Keijzer \\ Deborah de Groot \\ Charikleia Schoina \\ Klaas Bouwmeester \\ Tijs Ketelaar \\ Francine Govers
}




\section{Abstract}

Live-cell imaging of plant-pathogen interactions is often hampered by the tissue complexity and multi-cell layered nature of the host. Here, we established a novel pathosystem with the moss Physcomitrella patens as host for Phytophthora. The tip-growing protonema cells of this moss are ideal for visualizing interactions with the pathogen over time using high-resolution microscopy. We tested four Phytophthora species for their ability to infect $P$. patens and showed that $P$. sojae and $P$. palmivora were only rarely capable to infect $P$. patens. In contrast, $P$. infestans and $P$. capsici frequently and successfully penetrated moss protonemal cells, showed intracellular hyphal growth and formed sporangia. Next to these successful invasions, many penetration attempts failed. Here the pathogen was blocked by a barrier of cell wall material deposited in papilla-like structures, a defence response that is common in higher plants. Another common response is the upregulation of defencerelated genes upon infection and also in moss we observed this upregulation in tissues infected with Phytophthora. For more advanced analyses of the novel pathosystem we developed a special set-up that allowed live-cell imaging of subcellular defence processes by high-resolution microscopy. With this setup, we revealed that Phytophthora infection of moss induces repositioning of the nucleus, accumulation of cytoplasm and rearrangement of the actin cytoskeleton, but not of microtubules. 


\section{Introduction}

Plant pathogens cause enormous crop losses worldwide and are often difficult to control. For designing novel control strategies, we need to gain more in depth knowledge on molecular and subcellular plant defence processes. Upon pathogen recognition, plant cells initiate a dramatic reprogramming to prevent penetration. This results, for example, in the production of anti-microbial compounds, movement of organelles, reinforcement of cell walls or rearrangement of the cytoskeleton (Schmelzer, 2002; Hardham, 2007; Robatzek, 2007). Over the last decades, advanced methods for imaging cell biological processes have been developed and were used to visualize subcellular rearrangements upon infection of plants by biotrophic fungi and oomycetes (Schmelzer, 2002; Hardham, 2007; Huckelhoven \& Panstruga, 2011; Ben Khaled et al., 2015). Often these studies make use of fixed plant tissue which has as major drawback that the dynamics of the plant-pathogen interaction cannot be captured. Preferably, live-cell imaging is used, but here the drawback is the multi-cell layered nature of the host that hampers high-resolution microscopy. Unlike higher plants, the moss Physcomitrella patens is ideal for high-resolution microscopy. Since this moss is also known to be susceptible to a variety of pathogens, we set out to further exploit $P$. patens as a model host plant. In this study, we first tested the ability of the oomycete pathogen Phytophthora to infect $P$. patens and subsequently developed a livecell imaging set-up to investigate the host-pathogen interactions at the subcellular level.

The genus Phytophthora belongs to the class of oomycetes and comprises over 130 species that are all pathogens on a wide variety of plants (Kroon et al., 2012). Notorious species are Phytophthora infestans, the causal agent of potato and tomato late blight (Fry, 2008) and Phytophthora sojae, the causal agent of stem rot and root rot in soybean. Examples of broad host range species are Phytophthora capsici, that infects amongst others tomato and pepper (Lamour et al., 2012), and P. palmivora that is pathogenic on tropical crops such as cacao and date palm. For asexual reproduction, Phytophthora produces sporangia that can either germinate directly or develop into zoosporangia. The latter release motile zoospores that first encyst upon touching a surface and then germinate. At the germ tube tip an appressorium is formed that enables host cell penetration. In higher plants the mycelium grows in between the mesophyl cells and forms feeding structures called haustoria that invade the cells. When the lesion expands, sporangiophores emerge through the stomata and the sporangia that are formed can start a new infection cycle (Hardham, 2001; Judelson \& Blanco, 2005).

P.patens is a leafy bryophyte growing in many temperate regions worldwide. The bryophytes diverged (450 million years ago) from a common ancestor shared with vascular plants and thus forms an evolutionary link between green algae and vascular plants (Knight et al., 2009). During its life-cycle, P. patens is largely haploid with only a very short diploid, sporophytic phase. Upon germination, the haploid spores form a filamentous network called protonema and this protonema differentiates into gametophores with small leaflike phyllidia and rhizoids. After fertilization a diploid sporophyte develops on top of these gametophores that undergoes meiosis to produce the next generation of haploid spores 
(Cove, 2005). P. patens has become an important model system for functional genomics and cell biology. It is easily cultured and its highly efficient homologous recombination allows direct gene targeting (Nishiyama et al., 2000). Moreover, the fact that protonema and phyllidia are one-cell-layer-thick, makes P. patens ideal for microscopy.

In nature, mosses are infected by microbial pathogens often leading to severe damage (Davey \& Currah, 2006; Davey et al., 2009). To exploit the advantages of P. patens as a model host plant, several pathogens have been tested for their ability to infect $P$. patens. For the fungal pathogens Botrytis cinerea, Altenaria brassicicola, Altenaria alternata, Fusarium avenaceum and Fusarium oxysporum (Ponce de Leon et al., 2007; Akita et al., 2011; Bressendorff, 2012; Lehtonen et al., 2012b; Ponce De Leon et al., 2012), the bacterium Pectobacterium carotovorum (Andersson et al., 2005; Ponce de Leon et al., 2007) and three species of the oomycete genus Pythium (Oliver et al., 2009; Takikawa et al., 2015), it has been shown that they are capable to infect $P$. patens. More recently, Reboledo et al. (2015) described the infection of $P$. patens by the hemi-biotrophic pathogen, Colletotrichum gloeosporioides. These studies revealed that these pathogens cause extensive cell death of moss tissue and induce defence responses in P. patens that are similar to those activated in flowering plants (Ponce de León, 2011; Ponce de León \& Montesano, 2013). For example, inoculation with the Pythium species, B. cinerea and C. gloesporioides resulted in cell wall reinforcement and the production of reactive oxygen species (ROS) (Oliver et al., 2009; Ponce De Leon et al., 2012; Reboledo et al., 2015) and exposing $P$. patens tissue to the fungal elicitors chitin and chitosan lead to a rapid ROS burst, increased peroxidase activity and induction of chitinase secretion (Lehtonen et al., 2009; Lehtonen et al., 2012a; Lehtonen et al., 2014). Furthermore, levels of the hormone salicylic acid (SA) and a precursor of jasmonic acid (JA) were found to be increased upon $B$. cinerea infection (Ponce De Leon et al., 2012). Also, several genes encoding defencerelated proteins were found to be up-regulated upon pathogen attack or elicitor treatment (Oliver et al., 2009; Bressendorff, 2012; Lehtonen et al., 2012a; Ponce De Leon et al., 2012; Reboledo et al., 2015). These included genes encoding phenylalanine ammonia lyase (PAL) and chalcone synthase (CHS), both enzymes in the flavonoid pathway that produces antimicrobial compounds and is involved in SA accumulation (Huang et al., 2010; Dao et al., 2011), and a lipoxygenase (LOX) that is part of the oxylipin pathway of which JA is one of the products (Porta \& Rocha-Sosa, 2002). Also genes encoding the superoxide producer NADPH oxidase (NOX), (Sagi \& Fluhr, 2006), the mitogen activated protein kinase 4a (MPK4A) that in A. thaliana is involved in early defence signalling (Berriri et al., 2012) and the transcription factor ethylene-responsive element-binding factor 5 (ERF5) were up-regulated. Together, these findings indicate that basal defence signalling was already present prior to the emergence of higher plants. Once penetration in higher plants has been achieved, the pathogen can still be stopped by a local hypersensitive response (HR) which is triggered by resistance proteins that recognize pathogen effectors (van Ooijen et al., 2007). The genome of $P$. patens contains several resistance gene homologs (Akita \& Valkonen, 2002; Xue et al., 2012; Tanigaki et al., 2014), but as yet activation of HR in moss has not been reported. 
In this study, we describe a novel pathosystem with P. patens as host for two Phytophthora species. We characterized the infection of $P$. patens by $P$. infestans and $P$. capsici and analysed defence responses upon attack by these pathogens, including cell wall fortification and the induction of defence-related gene expression. We also demonstrate the high potential of this pathosystem for high-resolution live-cell imaging of subcellular defence by visualizing local reorganisation of the actin cytoskeleton in P. patens upon Phytophthora infection.

\section{Material and methods}

\section{P. patens culturing conditions}

P. patens wild-type isolate Gransden (Ashton \& Cove, 1977) was routinely cultured on BCDAT or BCD agar (Nishiyama et al., 2000) at $25^{\circ} \mathrm{C}$ under continuous white light.

\section{Transformation of $\boldsymbol{P}$. patens}

To create a moss line in which both actin filaments and microtubules are fluorescently labelled, a LifeAct-eGFP construct (Vidali et al., 2009) was transformed into a moss line expressing a gene encoding mCherry fused to a-tubulin driven by the rice actin promoter (McElroy et al., 1990) and integrated at the HB7 locus (Hiwatashi et al., 2008) (T. Miki, unpublished). Transformation was performed using PEG-mediated protoplast transfection (Nishiyama et al., 2000) and stable transformants were selected by resistance to hygromycin after a period of release from selection.

TABLE 1: Phytophthora isolates used in this study.

\begin{tabular}{|c|c|c|c|}
\hline $\begin{array}{l}\text { Phytophthora } \\
\text { species }\end{array}$ & Isolate & Origin & Reference \\
\hline P. infestans & 14-3-GFP & GFP-expressing transformant of $P$. infestans $\mathrm{H} 30 \mathrm{P} 02$ & - \\
\hline \multirow[t]{2}{*}{ P. capsici } & LT263 & Isolated from pumpkin & Donahoo \& Lamour, 2008 \\
\hline & LT3239 & Isolated from pumpkin & - \\
\hline P. sojae & P6497 & Isolated from soybean & Forster et al., 1994 \\
\hline P. palmivora & GFP3 & GFP-expressing transformant of P. palmivora P6390 & Vijn \& Govers, 2003 \\
\hline
\end{tabular}

\section{Pathogen growth conditions and inoculum preparation}

Isolates of P. capsici, P. palmivora and P. sojae (Table 1) were cultured on $20 \%$ (v/v) V8 juice agar at $25^{\circ} \mathrm{C}$ under continuous light (Erwin \& Ribeiro, 1996). P. infestans was cultured on rye sucrose agar at $18^{\circ} \mathrm{C}$ in the dark (Caten \& Jinks, 1967). To produce inoculum, 11-14-daysold colonies of $P$. infestans were flooded with sterilized cold water, followed by incubation at $4^{\circ} \mathrm{C}$ for 3 hrs in the dark. Zoospores of $P$. palmivora were obtained by flooding 5-7-daysold colonies with sterilized cold water, followed by $30 \mathrm{~min}$ incubation at $25^{\circ} \mathrm{C}$ in light. $\mathrm{A}$ 


\section{Chapter 3}

similar method was used to isolate zoospores of $P$. capsici, with the difference that plates were unsealed and grown under continuous light to produce sporangia. Zoospores were filtered through a $0.5 \mu \mathrm{m}$ filter and concentrations were adjusted to $1 \times 10^{5}-1 \times 10^{6}$ zoospores $/ \mathrm{ml}$ using a haemocytometer. Inoculation with $P$. sojae was done with mycelial plugs of $0.3 \mathrm{~cm}$ in diameter from the edge of a 5 day-old colony.

\section{Trypan blue staining}

P. patens was grown on BCD agar overlaid with cellophane for 2-3 weeks and inoculated with a $50 \mu \mathrm{l}$ droplet of inoculum containing $1 \times 10^{5}$ zoospores $/ \mathrm{ml}$ per colony. Inoculated moss tissue was stained in trypan blue solution $(10 \mathrm{ml}$ phenol, $10 \mathrm{ml}$ glycerol, $10 \mathrm{ml}$ lactic acid, $10 \mathrm{ml}$ water, $0.02 \mathrm{~g}$ trypan blue) overnight and de-stained with chloral hydrate for at least 1 day (Wilson \& Coffey, 1980).

\section{P. patens growth and inoculation for live-cell imaging of infected cells}

Protonemal tissue was grown in a glass-bottom microwell dish of $35 \mathrm{~mm}$ in diameter (MatTek, Ashland, USA). This dish was first filled with BCD 1,2\% agar (w/v), subsequently a $1 \times 1 \mathrm{~cm}$ agar square was removed from the coverglass of the dish and a small moss explant was placed on the bottom. This explant was covered by a $1 \mathrm{~mm}$ thin layer of BCD $0.7 \%$ low melting point agar ( $\mathrm{w} / \mathrm{v}$ ) and protonemal tissue was grown under standard conditions for 5-7 days prior to inoculation. Subsequently, a $20 \mu$ droplet of inoculum containing in total $1 \times 10^{6}$ zoospores/ml was placed on top of protonemal tissue. Inoculated dishes were sealed with surgical micropore tape (3M Health Care Business, Neuss, Germany) and placed at the bench at room temperature for at least 4 hours before microscopic observation.

\section{Microscopy}

Brightfield microscopy was performed on a Nikon 90i microscope (Nikon, Amstelveen, The Netherlands) using differential interference contrast and a 60x water immersion 1.20 NA objective. Confocal fluorescence microscopy was performed using a Roper Spinning Disc Confocal Microscope (Nikon Ti microscope body, Yokogawa CSUX1-spinning disc head, Photometrics Evolve camera, Metamorph software, 491 and $561 \mathrm{~nm}$ laser lines; GFP filter 495-560 nm; mCherry filter 570-620 nm; 100x oil immersion 1.40 NA objective; $1.2 \mathrm{x}$ magnification lens between spinning disk head and camera). Z-stacks were acquired with an internal Z-spacing of $0.5 \mu \mathrm{m}$ and image processing was performed using ImageJ software.

\section{Quantitative RT-PCR}

P. patens protonemal tissue was homogenized with a polytron homogenizer, plated on BCDAT agar overlaid with cellophane and grown for 1 week. Subsequently, moss plates were sprayed with in total $1 \mathrm{ml}$ of $1 \times 10^{6}$ zoospores $/ \mathrm{ml}$ per $9 \mathrm{~cm}$ plate. Inoculated moss plates were sealed with surgical micropore tape (3M Health Care Business, Neuss, Germany) and placed at $25^{\circ} \mathrm{C}$ under continuous light. Mock inoculations were performed using sterilized 
deionized water. Total RNA was isolated from infected and mock-treated moss tissue by a Quick-RNA MiniPrep kit (Zymo Research, CA, USA). Synthesis of CDNA was performed on $2 \mu \mathrm{g}$ of total RNA using a M-MLV reverse transcriptase kit (Promega, Madison, USA). Q-RTPCR was performed using a SYBR Hi-ROX kit (Bioline, London, UK), gene-specific primers (Table 2) and $3 \mu$ of 10-times diluted cDNA using a Bio-Rad 7300 Real Time PCR system (Applied Biosystems, NY, USA). Gene expression levels were normalized to EF1 a transcript levels and to the transcript levels of the studied gene in mock-inoculated moss tissue.

TABLE 2. Primers used in this study.

\begin{tabular}{|c|c|c|c|}
\hline Gene & Gene ID* & Forward (Fw) and reverse (Rv) primer & Reference \\
\hline \multirow{2}{*}{ PpPAL4 } & \multirow{2}{*}{ Pp1s500_4V6.1 } & Fw: TGGCCTACTCGGTAATGGAG & \multirow{2}{*}{ Bressendorff, 2012} \\
\hline & & Rv: GTCAACCATCCGCTTGATTT & \\
\hline \multirow{2}{*}{ PpLOX7 } & \multirow{2}{*}{ Pp1s70_182V6.1 } & Fw: GTGGCGGTTTGATCAGGA & \multirow{2}{*}{ Lehtonen et al., $2012 \mathrm{a}$} \\
\hline & & Rv: CGTTCAGCCATCCCTCTTC & \\
\hline \multirow{2}{*}{ PpNOX } & \multirow{2}{*}{ Pp1s18_194V6.1 } & Fw: CACGATGTTGCAGTCGTTG & \multirow{2}{*}{ Lehtonen et al., $2012 \mathrm{a}$} \\
\hline & & Rv: TACGTGCCCTAGTGCCTGA & \\
\hline \multirow{2}{*}{ PpCHS } & \multirow{2}{*}{ Pp1s22_4V6.1 } & Fw: GGCATGGAACGAGATGTTCT & \multirow{2}{*}{ Bressendorff, 2012} \\
\hline & & Rv: CCTTGCATCTTGTCCTTGGT & \\
\hline \multirow{2}{*}{ PpERF5 } & \multirow{2}{*}{ Pp1s2_410V6.1 } & Fw: GCTCCGCTGTATCGAAAGTC & \multirow{2}{*}{ Bressendorff, 2012} \\
\hline & & Rv:TCGAAGTTGCTGACAAGGTG & \\
\hline \multirow{2}{*}{ РpMPK4A } & \multirow{2}{*}{ Pp1s149_39V6.1 } & Fw: GGTACAAGCCACCACTTCGT & \multirow{2}{*}{ Bressendorff, 2012} \\
\hline & & Rv: GGTCCGTATCCATCAACTCG & \\
\hline \multirow{2}{*}{ PpEF1a } & \multirow{2}{*}{ Pp1s163_112V6.2 } & FW: AATCATACATTTCACCTCGCC & \multirow{2}{*}{ Le Bail et al., 2013} \\
\hline & & Rv: GATCAGTGGGTAGAAGTGAC & \\
\hline
\end{tabular}

* Gene IDs used by www.cosmoss.org.

\section{Results and discussion}

\section{P. infestans and $P$. capsici are able to infect $P$. patens}

As a first step, we tested four Phytophthora species for their ability to infect $P$. patens. Moss colonies were inoculated with zoospores of $P$. infestans, $P$. capsici, P. palmivora and P. sojae and stained with trypan blue to visualize mycelium or spores of the pathogen and dead or damaged cells of the moss.

Inoculation with $P$. sojae rarely resulted in infection of $P$. patens. Only occasionally we observed local cell death reminiscent of successful colonization. Sometimes we also found oospores (i.e. sexual spores) inside cells of phyllidia and on protonemal tissue demonstrating that $P$. sojae is capable to grow and expand in moss (Figure 1A). P. sojae is homothallic and during interaction with its natural host soybean it readily produces oospores inside infected tissues (Erwin \& Ribeiro, 1996). Also P. palmivora was hardly able to infect moss cells (Figure 1B). Local cell death, cell wall browning and intracellular hyphal growth were observed in gametophore phyllidia cells. At a later stage of infection, i.e. 3 days post inoculation (dpi), 
sporangia were formed on moss tissue. Since infection by either $P$. sojae or $P$. palmivora was rarely observed, these two species were not used for further study.

Inoculation with the other two Phytophthora species did result in more frequent infections and browning of moss tissue, indicating that $P$. patens is susceptible to these pathogens (Figure 2A). Upon inoculation with both P. infestans and P. capsici, appressoria were formed that enabled penetration of moss cells and we observed hyphae growing inside these cells (Figure $2 \mathrm{~B} ; \mathrm{C}$ ). Compared to P. infestans, P. capsici grew faster and showed more penetration attempts even with similar amounts of zoospores in the inoculum. The intracellular growing hyphae often showed a dark brown coloration. Brown deposits surrounding penetrating hyphae have also been observed during the interaction of $P$. infestans with the non-host plant species Arabidopsis thaliana (Vleeshouwers et al., 2000) and in another moss, Funaria hygrometrica, infected by a fungal pathogen (Martinez-Abaigar et al., 2005; Davey et al., 2009; Davey et al., 2010). This dark brown coloration is reminiscent of the deposition of phenolic compounds, a general defence response observed in higher plants (Vleeshouwers et al., 2000).

A 5 dpi
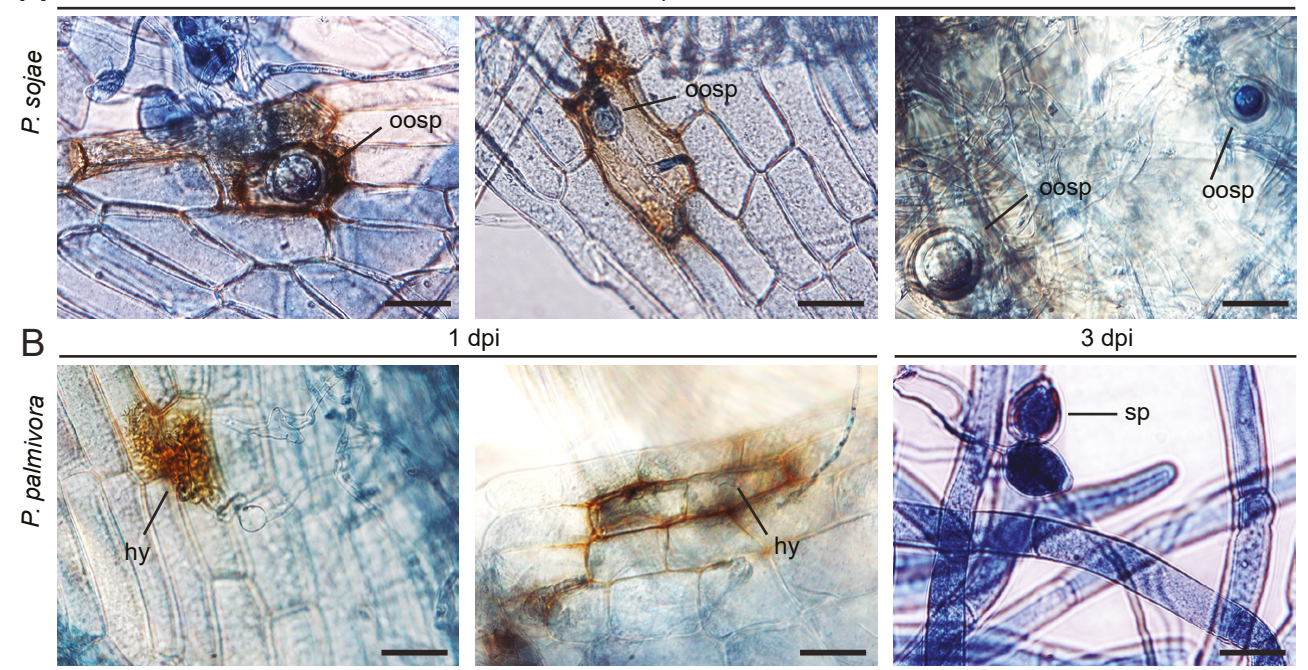

$1 \mathrm{dpi}$

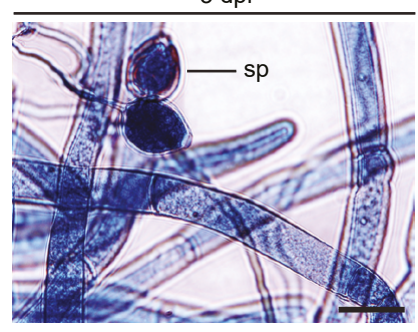

FIGURE 1. P. sojae and $P$. palmivora occasionally infect $P$. patens. P. patens at 5 days post inoculation (dpi) with P. sojae (A) and at 1 and 3 dpi with P. palmivora (B). The tissue is was stained with trypan blue and visualised by brightfield microscopy. Scale bars represent $20 \mu \mathrm{m}$. hy = intracellular hypha; oosp = oospore; $\mathrm{sp}=$ sporangium.

Besides successful penetration of moss cells, we also observed the formation of papillalike structures that blocked hyphal penetration and arrested further pathogen colonization (Figure $2 \mathrm{~B} ; \mathrm{C}$ ). In fact, roughly half of the penetration attempts were blocked by these papillalike structures. Papillae are cell wall depositions that are formed by higher plants as part of the first line of defence against fungal and oomycete pathogens (Schmelzer, 2002; Collinge, 2009). Papillae consist of callose, proteins, phenolic compounds and reactive oxygen species and physically resist hyphal penetration (Collinge, 2009). Papilla-like structures in mosses 
have been reported before. In P. patens they were found upon inoculation with the nonvirulent fungus Apiospora montangei (Lehtonen et al., 2012b) and in Funaria hygrometrica when challenged with Atradidymella muscivora and Coniochaeta velutina (Davey et al., 2009; Davey et al., 2010). Lehtonen et al. (2012b) showed that the papilla-like structures in P. patens can be stained with aniline blue, indicating that they contain glucans.

During a later stage of infection, sporangia were detected (Figure 2 B; C), demonstrating that the disease-cycle of $P$. infestans and $P$. capsici can be completed on P. patens. However, we have never observed a typical haustorium and combined with the observed defence responses, e.g. the deposition of phenolic compounds and the formation of papilla-like structures, we conclude that the interactions between $P$. patens and either $P$. infestans or $P$. capsici resemble non-host interactions. We therefore consider this pathosystem useful to study basal plant defence responses upon pathogen attack and in particular at early stages of the interaction. Because $P$. capsici shows a higher infection efficiency than $P$. infestans, P. capsici was used to analyse this pathosystem in more depth.

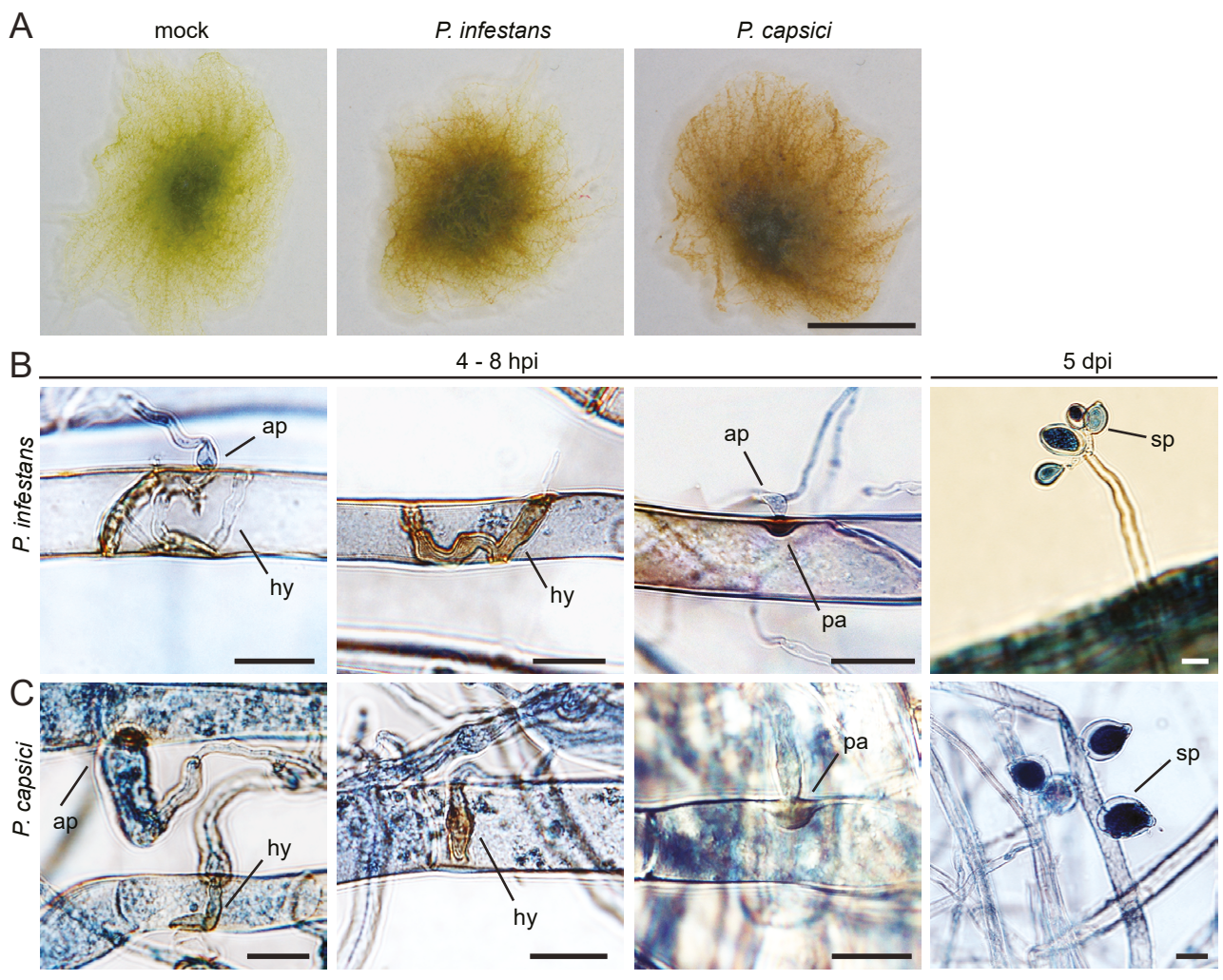

FIGURE 2. $\boldsymbol{P}$. infestans and $\boldsymbol{P}$. capsici are able to infect $\boldsymbol{P}$. patens. (A) Moss colonies at 2 dpi with zoospores of $P$. infestans and $P$. capsici, and mock treatment. Scale bar is $5 \mathrm{~mm}$. (B,C) The interaction between $P$. patens and P. infestans (B) or P. capsici (C) at 4-8 hpi and $5 \mathrm{dpi}$. The tissue was stained with trypan blue and visualised by brightfield microscopy. Scale bars represent $20 \mu \mathrm{m}$. ap = appresorium; hy = intracellular hypha; pa = papilla-like structure; $\mathrm{sp}=$ sporangium. 


\section{Defence-related genes of $\boldsymbol{P}$. patens are up-regulated upon Phytophthora infection}

The overall defence response is usually accompanied with transcriptional reprogramming of defence-related genes (Dodds \& Rathjen, 2010). To investigate if this is also the case in the P. patens - Phytophthora pathosystem, we studied the expression of six P. patens defence-related genes upon P. capsici infection, i.e. PAL4, LOX7, NOX, MPK4, CHS and ERF5. One-week old protonema cultures were inoculated with zoospores of $P$. capsici and total RNA was isolated 12 hours later. Transcript levels were analysed by qRT-PCR and normalised to their levels in mock-treated protonema cells (Figure 3). The transcript levels of all studied genes increased upon infection, ranging from 8 up to 58 fold. This indicates that some of the common defence pathways of higher plants are also activated in P. patens upon infection with Phytophthora. This is in line with findings in earlier studies dealing with P. patens infected with Botrytis, Pythium or Colletotrichum or treated with chitosan (Oliver et al., 2009; Bressendorff, 2012; Lehtonen et al., 2012a; Ponce De Leon et al., 2012; Reboledo et al., 2015).

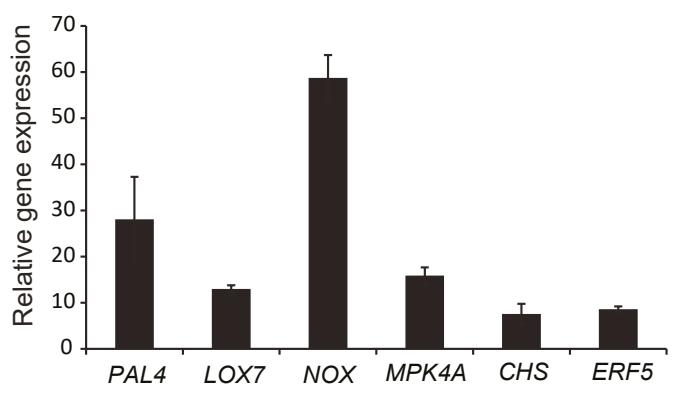

FIGURE 3. Defence-related genes are up-regulated upon $\boldsymbol{P}$. capsici infection. Transcript levels of six defencerelated $P$. patens genes at $12 \mathrm{hpi}$ were analysed by qRT-PCR and normalized to the endogenous $P$. patens gene EF1a. The $x$-axis shows the mean fold changes ( \pm standard deviation of 3 technical replica's) relative to the transcript level in moss tissue 12 hours after mock treatment that was set at 0 . This experiment was repeated at least three times with comparable outcomes.

\section{A set-up for live-cell imaging of moss-pathogen interactions}

For high-resolution live-cell imaging of moss cells during pathogen attack, we developed a special set-up that allowed us to image the Phytophthora-Physcomitrella interaction over a relatively long time frame (Figure 4). In this set-up we used prefabricated glassbottom microwell dishes containing a coverglass as bottom. A moss explant was placed on the glass bottom of the dish, covered with a thin layer of BCD agar and allowed to grow for 5-7 days. In this way, the protonemal tissue grows close to the coverglass and can be maintained in optimal conditions for several days. Most importantly, the glassbottom microwell dish can be placed directly on the stage of a microscope, so for imaging, the protonema does not have to be disrupted for transfer to a microscope slide. The protonema was inoculated by pipetting a $20 \mu \mathrm{l}$ droplet of inoculum containing in total $2 \times 10^{4}$ zoospores on top of the thin agar layer. After incubation for at least 4 hours in the 
light, the dish was placed on the stage of an inverted microscope for microscopic analysis. Zoospores germinated and hyphae grew through the thin layer of agar on top of the moss tissue. Pipetting zoospores beneath the thin agar layer by pressing the pipette tip against the coverglass yielded even more penetration attempts.

We have tried several imaging set-ups, but the one we describe here was most effective for imaging Physcomitrella-Phytophthora interactions. This method proved the most effective, as the moss tissue did not have to be transplanted between infection and observation. The delicate protonema cells are easily stressed or even die when transferred and this influences their defence capacity. This was illustrated by the inability of $P$. patens to form papilla-like structures when the moss tissue was lifted and placed on top of Phytophthora zoospores.

\section{Live-cell imaging reveals local actin accumulation upon Phytophthora infection}

To demonstrate the power of this set-up for live-cell imaging of plant defence responses, we studied cytoskeletal reorganisation during infection. To easily visualize the cytoskeleton, we generated a transgenic moss line expressing both LifeAct-GFP, a fluorescent marker that binds to filamentous actin (Riedl et al., 2008), and mCherry-tua1, a fusion protein of a-tubulin and the fluorescent tag mCherry. Lifeact-GFP has been used in Physcomitrella previously (Vidali et al., 2009). Since overexpression of Lifeact-GFP is known to cause growth defects (Vidali et al., 2009) and inhibits reorganisation of actin filaments in plants (Van der Honing et al., 2011), we carefully screened transgenic moss lines for abnormalities. The line we used behaved similar to wild-type $P$. patens and showed no abnormal growth behaviour or developmental defects.
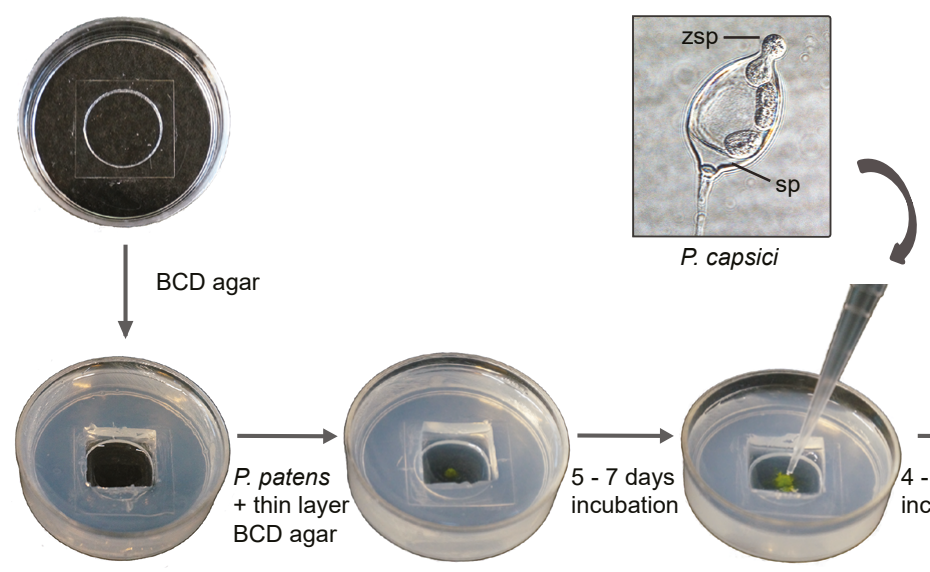

FIGURE 4. Set-up for high-resolution live-cell imaging of infected moss cells. A glass-bottom microwell dish (diameter $35 \mathrm{~mm}$ ) is filled with BCD agar and a square of agar is removed from the middle of the dish. A small explant of $P$. patens is placed in the middle, covered with a thin layer of BCD agar and allowed to grow for 5-7 days. Zoospores (zsp) of $P$. capsici released from sporangia (sp) are used as inoculum. The inoculum is added to the moss tissue by pipetting. After 4 hours the first infection attempts can be observed by microscopy. 

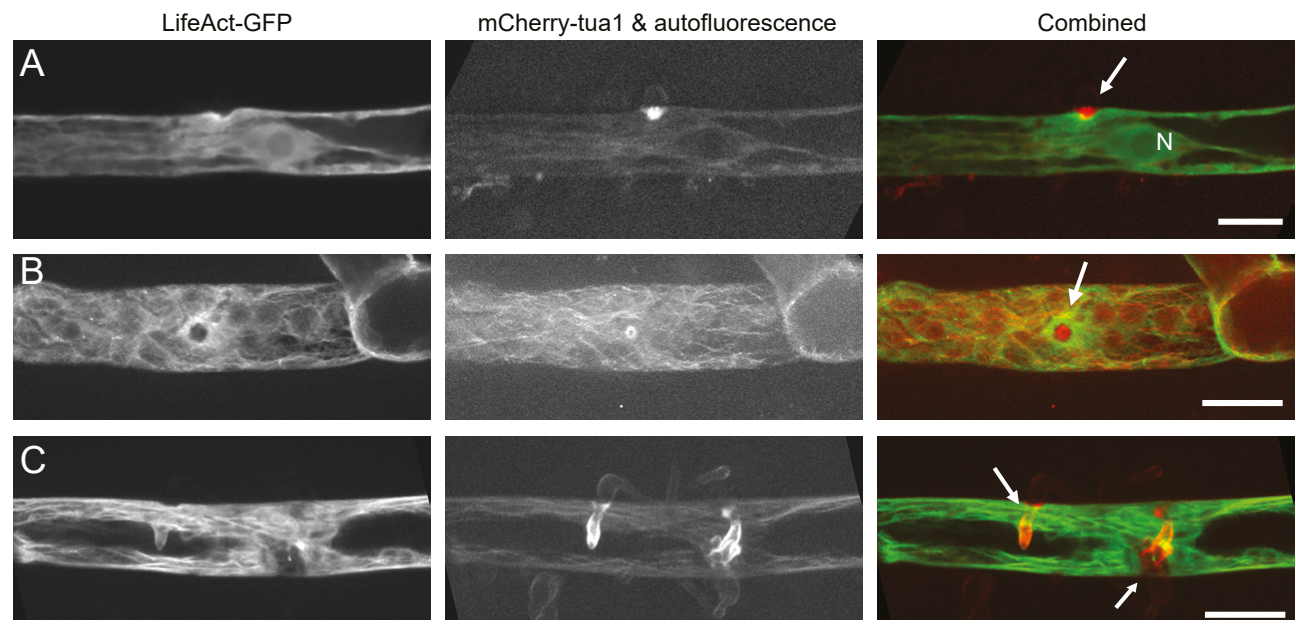

FIGURE 5. Subcellular cytoskeleton rearrangements in $\boldsymbol{P}$. patens protonema cells upon $\boldsymbol{P}$. capsici infection. Confocal fluorescent microscopy images of the actin and microtubule cytoskeleton in a transgenic moss line with LifeAct-GFP and mCherry-tua1 at $5 \mathrm{hpi}$ with $P$. capsici. (A) Average Z-projection $(1.5 \mu \mathrm{m})$ of side view, (B) maximum Z-projection $(11 \mu \mathrm{m})$ of top view and (C) maximum Z-projection (8.5 $\mu \mathrm{m})$ of side view. Arrows point to the site of pathogen attack and scale bars represent $10 \mu \mathrm{m} . \mathrm{N}=$ nucleus.

As shown in Figure 5, the location of the penetration attempt is easily recognised by the strong auto-fluorescence of papilla-like structures when excited by the $561 \mathrm{~nm}$ laser line or, in case of successful penetration, by the deposited material around the invasive hyphae. We often observed accumulation of cytoplasm around the penetration site with the nucleus in close proximity (Figure 5A). Immediately upon penetration and around the papilla-like structures, we observed a rapid accumulation of actin filaments around the site of attack (Figure $5 \mathrm{~A} ; \mathrm{B}$ ), but at later stages, after successful penetration of a moss cell by $P$. capsici, the actin cytoskeleton did no longer accumulate around the infection site (Figure 5C). Similar responses were observed in higher plants, where cytoplasmic accumulation and nuclear repositioning are regarded as basal defence responses upon hyphal penetration attempts (Gross et al., 1993; Schmelzer, 2002; Takemoto \& Hardham, 2004; Koh et al., 2005; Hardham, 2007; Huckelhoven \& Panstruga, 2011) and where the actin cytoskeleton was found to be essential for proper basal defence against fungal and oomycete pathogens (Takemoto \& Hardham, 2004; Hardham et al., 2007; Schmidt \& Panstruga, 2007; Day et al., 2011). It has been shown for example that disrupting the actin cytoskeleton by actin depolymerising drugs leads to an increased susceptibility to pathogen attack (Kobayashi et al., 1997; Kobayashi \& Hakuno, 2003; Yun et al., 2003; Jarosch et al., 2005; Miklis et al., 2007). Actin accumulation has been observed during both incompatible and compatible interactions and this accumulation has been hypothesised to facilitate vesicle delivery to the infection site (Gross et al., 1993; Takemoto et al., 2003; Opalski et al., 2005; Takemoto et al., 2006; Humbert et al., 2015). Even treatment with fungal elicitors increased actin abundance through the whole cell (Henty-Ridilla et al., 2013; Henty-Ridilla et al., 2014; Li et al., 2015) and mimicking the pressure generated by a penetrating pathogen by touching a 
plant cell with a glass microneedle, resulted in a rapid accumulation of actin at the site of touch (Hardham et al., 2008). This suggests that the changes in actin cytoskeleton that are triggered upon pathogen recognition are both due to molecular cues produced directly or indirectly by the pathogen and due to physical encounter with the pathogen.

In contrast to the actin cytoskeleton, we did not observe a clear change in microtubule organization in P. patens upon infection by Phytophthora (Figure 5B). During interphase, cortical microtubules function in guiding cellulose synthase complexes to facilitate cellulose microfibril deposition (Paredez et al., 2006). The behaviour of the microtubule cytoskeleton in response to pathogen infection is variable and its role in plant defence remains unclear (Hardham, 2013). Often, pathogen attack induces depolymerisation of microtubules at the site of attack (Gross et al., 1993; Baluska et al., 1995; Cahill et al., 2002; Takemoto et al., 2003; Takemoto et al., 2006) a phenomenon that is also observed upon touching with a microneedle (Hardham et al., 2008). Treating plants with microtubule depolymerizing drugs had less effect on defence ability than depolymerizing actin (Hardham, 2013), but did lead to increased susceptibility to a non-host pathogen in wheat (Li et al., 2010).

Taken together, we showed that cytoplasmic accumulation, nuclear repositioning and actin accumulation upon pathogen penetration are basal subcellular defence processes that were already present in lower plants and that this novel imaging set-up is suitable to visualize subcellular plant defence processes by high-resolution microscopy.

\section{Live-cell imaging of plant-pathogen interactions in perspective}

One of the major obstacles for live-cell imaging of plant-pathogen interactions is caused by the fact that often these interactions take place in a sub-epidermal cell layer of the plant leaf, and this hampers the collection of fluorescent signals by microscopy due to the large working distance and light scattering. One way to solve this is by making sections of the infected plant tissue. This was done in a recent live-cell imaging study in which the authors imaged the interaction between Magnaporthe oryzae and rice after removing the upper epidermis of the leaf with scissors (Mochizuki et al., 2015). However, this type of manipulation likely induces stress to the plant cells and hence may interfere with a correct interpretation of the observations. A major advantage of $P$. patens is the singlecelled nature of the protonema tissue that allows visualization of the plant-pathogen interaction with high-resolution microscopy over time, without the need for manipulation prior to imaging.

Another obstacle is the microscopic visualization of plant proteins. A frequently used approach is transient expression of genes encoding fluorescently-tagged proteins using Agrobacterium infiltration and subsequently challenging of the infiltrated leaves with pathogens. The location and behaviour of the tagged proteins in response to pathogen infection can then be monitored by fluorescence microscopy. This approach has, for example, been used for live-cell imaging of proteins involved in endocytic trafficking Nicotiana benthamiana and then in particular to monitor their behaviour in response to $P$. 
infestans infection (Lu et al., 2012). Although this approach is efficient and quickly yields results, it also has serious disadvantages. First of all, agroinfiltration often triggers plant defence responses and that might by itself influence the behaviour of the tagged proteins that are the subject of study. Secondly, the use of constitutive and strong promoters to drive the expression of genes encoding the fluorescently-tagged proteins often leads to overexpression or expression at the wrong time or at the wrong place. This might cause artefacts that change the localization or dynamics leading to wrong interpretations. The advantage of $P$. patens is its convenient toolbox of gene modification. The highly efficient homologous recombination allows direct integration of fluorescent tags in the target gene at the endogenous locus in the genome (Nishiyama et al., 2000). In this way the timing and level of expression of the modified genes is the same as in the wild-type situation and this is especially important when studying proteins that function in a fixed stoichiometry with other proteins or are highly dynamic. The latter certainly applies to subcellular defence response upon pathogen attack.

\section{Outlook}

In this study, we developed a novel live-cell imaging set-up to study subcellular defence responses during interactions between the moss $P$. patens and the hemi-biotrophic pathogen Phytophthora. We used this set-up to visualise the moss cytoskeleton during infection and showed that actin accumulates in P. patens around penetration sites, similar to what has been observed in higher plants. In the future, we will use the PhyscomitrellaPhytophthora pathosystem to study other subcellular defence responses, including exocytosis. This novel pathosytem is also suitable to visualize cellular processes in Phytophthora while it is interacting with its host. By using the various fluorescent marker strains of $P$. infestans including LifeAct-GFP transformants (Meijer et al., 2014) we can follow the infection process and the dynamics of the actin cytoskeleton in Phytophthora during infection in more detail.

\section{Acknowledgements}

We thank Tomohiro Miki from the Goshima lab (Nagoya University, Japan) for kindly providing the $P$. patens mCherry-tua1-HB7 line and Magdalena Bezanilla (University of Massachusetts, USA) for providing the LifeAct-eGFP construct. This research was supported by the Earth and Life Sciences Division and the Technology Foundation of The Netherlands Organization for Scientific Research (PhD grant E. O. and VENI grant K.B. respectively) and by the Wageningen University Fund 'Food-for-Thought campaign'. 


\section{Chapter 4}

Time-gated confocal microscopy reveals accumulation of exocyst subunits at the plant-pathogen interface

Elysa J.R. Overdijk Han Tang Jan W. Borst Francine Govers Tijs Ketelaar 


\section{Abstract}

Polarized exocytosis is essential for plant development and defence. The exocyst, an octameric protein complex that tethers exocytotic vesicles to the plasma membrane, targets exocytosis. Upon pathogen attack, secreted materials form papillae to halt pathogen penetration. To determine if the exocyst is directly involved in targeting exocytosis to infection sites, information about its localization is instrumental. Here, we investigated exocyst subunit localization in the moss Physcomitrella patens upon pathogen attack and infection by Phytophthora capsici. Time-gated confocal microscopy was used to eliminate autofluorescence of deposited material around infection sites allowing the visualization of the subcellular localization of exocyst subunits and of v-SNARE Vamp72A1-labeled exocytotic vesicles during infection. This showed that exocyst subunits Sec3a, Sec5b, Sec5d and Sec6 accumulated at sites of attempted pathogen penetration. Upon pathogen invasion, the exocyst subunits accumulated on the membrane surrounding papilla-like structures and hyphal encasements. Vamp72A1-labeled vesicles were found to localize in the cytoplasm around infection sites. The re-localization of exocyst subunits to infection sites suggests that the exocyst is directly involved in facilitating polarized exocytosis during pathogenesis. 


\section{Introduction}

Cellular defence processes play a key role in the arms race between plants and pathogens. These processes include targeted secretion of antimicrobial compounds and reinforcement of the cell wall with callose-rich material to prevent penetration. Both processes strongly depend on polarized exocytosis (Gu et al., 2017). Exocytosis is a multi-step process during which secretory vesicles are delivered to, and fuse with the plasma membrane (Matheson et al., 2006). This results in the secretion of vesicle content and incorporation of membrane lipids and membrane proteins into the plasma membrane. The final steps before membrane fusion involve tethering and docking at the target membrane followed by membrane fusion. The initial tethering of vesicles to the plasma membrane involves multi-subunit tethering factors, including the exocyst complex (Ravikumar et al., 2017). The tethered vesicle is tightly docked and subsequently fused with the plasma membrane by a SNARE (soluble $\mathrm{N}$-ethylmaleimide sensitive factor attachment protein receptor) complex formed between SNAREs present on the vesicle (v-SNAREs) and on the target membrane (t-SNAREs) (Duman \& Forte, 2003).

The exocyst is an evolutionary conserved protein complex consisting of eight subunits: Sec3, Sec5, Sec6, Sec8, Sec10, Sec15, Exo70 and Exo84 (Mei \& Guo, 2018). It has been extensively studied in the budding yeast Saccharomyces cerevisiae and animal cells. In yeast, the exocyst targets polarized exocytosis during bud growth and cytokinesis, and in animal cells, the exocyst plays a role in neuronal growth, synaptogenesis and cell motility (Heider \& Munson, 2012). In contrast to yeast and animals, plants often have multiple genes encoding the individual exocyst subunits (Cvrckova et al., 2012). The plant exocyst plays important roles in a variety of developmental processes, including cytokinesis, secondary cell wall deposition and polarized exocytosis during tip growth of pollen tubes and root hairs (Zarsky et al., 2013; Bloch et al., 2016; Martin-Urdiroz et al., 2016; Mayers et al., 2017; Sekeres et al., 2017; Kulich et al., 2018; Tang et al., 2019).

Polarized exocytosis is an important process during plant-microbe interactions. In plants challenged with beneficial microbes, such as rhizobia and mycorrhizae, it is essential for the formation of specialised membranes between the two partners. Several exocyst subunits are important in these beneficial interactions and localize at the plant-microbe interface (Genre et al., 2012; Zhang et al., 2015; Gavrin et al., 2017). Also during interactions with pathogenic microbes, polarized exocytosis is important (Gu et al., 2017), but the subcellular localization of exocyst subunits during pathogen infection has as yet not been studied.

In recent years, there is increased interest in the role of the exocyst in plant defence and to date there is ample evidence that proper defence requires a functional exocyst. Du et al. (2018) showed, for example, that reduced levels of particular exocyst subunits affect the defence against several pathogens. Nicotiana benthamiana plants producing low levels of Sec5, Sec6, Sec8, Sec10, Sec15A, Exo70B or Exo84B due to gene-silencing, showed enhanced susceptibility to the oomycete pathogen Phytophthora infestans. Similarly, silencing of Sec5, Sec6 and Sec10 enhanced susceptibility to the bacterium Pseudomonas syringae, but reduced lesion expansion of the necrotrophic fungus Botrytis 
cinerea. Moreover, callose deposition was reduced in nearly all silenced plants (i.e. 15 of the 17 tested exocyst subunits), suggesting the involvement of the exocyst in delivering material for raising a physical barrier (Du et al., 2018a). Studies in Arabidopsis thaliana have mainly focussed on the various Exo70 subunits, a family with 23 members of which several were found to play a role in plant defence (Martin-Urdiroz et al., 2016; Sabol et al., 2017). The fact that plants with silenced or mutated exocyst subunits show defects in papillae formation and callose deposition (Pecenkova et al., 2011; Kulich et al., 2015; Du et al., 2018a; Guo et al., 2018b; Kulich et al., 2018), hints to a possible role of the exocyst in polarized exocytosis of defence-related compounds. The central importance of the exocyst complex in plant defence is further illustrated by the fact that pathogen effectors can exploit exocyst subunits as host targets to modulate defence. Effector AVR-Pii from the rice blast fungus Magnaporthe oryzae interacts with rice Exo70F2 and -F3 and these two subunits are essential for blast resistance in rice mediated by the resistance protein Pii (Fujisaki et al., 2015). RXLR-effector AVR1 from the oomycete pathogen P. infestans targets potato Sec 5 and stabilizes it, presumably to disable Sec 5 thereby suppressing host defence (Du et al., 2015a).

The studies mentioned above are largely based on the phenotypic characterization of plants in which the production of specific exocyst subunits is decreased or abolished. Since these plants often show developmental defects, the question arises how one can distinguish between a direct or indirect effect of an aberrant exocyst. Is the exocyst directly involved in defence or is the impaired defence due to defects in the general metabolism? In case of a direct involvement, it is a long-standing hypothesis that the exocyst complex is recruited towards the site of pathogen penetration to promote defence-related vesicle tethering fusion. One way to test this hypothesis is by live cell imaging of plant tissue upon pathogen attack. However, this has proven difficult mainly because of the multicell layered nature of seed plants is often hampering high-resolution microscopy. To circumvent this we switched to the moss Physcomitrella patens which has protonema cells composed of a single cell layer. We established a new pathosystem with Physcomitrella patens as host for Phytophthora pathogens (Overdijk et al., 2016) and this facilitated the visualization of exocyst localization during pathogen infection. We exploited the efficient homologous recombination system in P. patens to introduce tags into endogenous genes and obtained a set of $P$. patens lines expressing GFP-tagged exocyst subunits including Sec3, Sec5 and Sec6 (Tang et al., 2019). P. patens has three Sec3 genes, four Sec5 genes and one Sec6 gene (Cvrckova et al., 2012; Tang et al., 2019). Microscopic analyses of four exocyst subunit lines with expression levels sufficient for microscopy, revealed localization of Sec3a, Sec5b, Sec5d and Sec6 to plasma membrane foci and accumulation of these subunits at the growing tip (Tang et al., 2019), which is consistent with previous observations in P. patens (van Gisbergen et al., 2018) and in seed plants (Fendrych et al., 2013; Zhang et al., 2013; Bloch et al., 2016; Vukasinovic \& Zarsky, 2016; Synek et al., 2017).

Although P. patens has been used by others as a model system to investigate evolutionary aspects of plant defence (Ponce de León \& Montesano, 2013; Bressendorff et al., 2016), we take advantage of its ideal morphology for high-resolution microscopy. Upon inoculation 
with Phytophthora capsici or Phytophthora infestans, highly autofluorescent material is deposited in papilla-like structures and around invasive hyphae (Overdijk et al., 2016), a phenomenon also observed in the Arabidopsis - P. infestans interaction (Vleeshouwers et al., 2000). The deposition of defence materials like callose, lignin, phenolic compounds and reactive oxygen species in papillae is a general response observed in seed plants (Collinge, 2009). The abundant presence of autofluorescent materials at infection sites makes it notoriously difficult to detect fluorescently tagged proteins that are weakly expressed. In order to eliminate this undesired autofluorescence we make use of time-gated confocal microscopy, an imaging technique that is able to differentiate between fluorescence signals based on their fluorescence life times (Kodama, 2016; Grossmann et al., 2018).

In this study, we use time-gated confocal microscopy and the P. patens lines with fluorescently tagged exocyst subunits, to visualize the subcellular localization of the exocyst during infection. We show that exocyst subunits Sec3a, Sec5b, Sec5d and Sec6 mark the plasma membrane underneath sites of pathogen penetration and that during later infection stages, they accumulate on the membrane surrounding papilla-like structures and hyphal encasements. These findings highlight the direct involvement of the exocyst in polarized exocytosis during plant defence.

\section{Materials and methods}

\section{Plasmids and cloning procedures}

All plasmids were generated using standard restriction-ligation cloning and verified by sequencing. Plasmids and primers used in this study are summarized in Supplementary Table S1 and S2.

peGFP-Nter: This plasmid is a modified version of the peGFP-NPTII vector (de Keijzer et al., 2017). The fluorophore was removed by a HindIII+EcoRI digestion and 2 dimerised primers (JK91 + JK92) containing additional restriction sites were used to re-ligate the linearized plasmid. The vector was finalised by a Nhel and Spel back-ligation to remove the NPTII cassette.

GFP-Vamp72A1: Regions of approximately $1 \mathrm{~kb}$ before and after the start codon of Vamp72A1 were amplified by PCR using primers listed in Supplementary Table S1. The PCR fragments were digested with the corresponding restriction enzymes and ligated into digested peGFP-Nter vector.

\section{P. patens growth conditions and transformation}

P. patens was routinely grown on plates containing BCDAT medium in continuous light at $25^{\circ} \mathrm{C}$ (Nishiyama et al., 2000). Plasmids were linearized and introduced into the P. patens genome by homologous recombination using PEG-mediated protoplast transformation (Nishiyama et al., 2000). Correct insertion events were characterised by PCR (Supplementary 
Figure S2). Characteristics of generated moss lines and their use throughout this study are summarized in Supplementary Table S3.

\section{Pathogen growth conditions, inoculum preparation and $\boldsymbol{P}$. patens inoculation}

Phytophthora capsici isolate LT263 was cultured on sealed Petri dishes containing $20 \%(\mathrm{v} / \mathrm{v})$ V8 juice agar at $25^{\circ} \mathrm{C}$ in the dark for 4 days. Plates were unsealed and zoospore production was induced under continuous light at $25^{\circ} \mathrm{C}$ for 3-4 days. Inoculum was harvested by flooding colonies with water, followed by 30 -min incubation at room temperature in light. Zoospores were filtered through a $0.5 \mu \mathrm{m}$ filter, counted with a haemocytometer and concentrations were adjusted to approximately $1 \times 10^{6}$ zoospores per $\mathrm{mL}$. For livecell imaging of infected cells, protonemal moss tissue was grown for 5 to 7 days in glassbottom dishes as described by Overdijk et al. (2016). Just before inoculation, the moss tissue was covered with an additional layer of BCD with $0.7 \%$ low melting point agar $(\mathrm{w} / \mathrm{v})$ and inoculated by pipetting $20 \mu \mathrm{l}$ zoospore solution between the moss tissue and the coverslip. Inoculated samples were incubated at room temperature in the light and used for microscopy 3-6 hours later.

\section{Fluorescence microscopy and FM4-64 staining of non-infected $\boldsymbol{P}$. patens cells}

Live-cell imaging of non-infected cells was performed on a spinning disk confocal microscope consisting of a Nikon Ti eclipse body equipped with a Yokogawa CSU-X1 spinning disc head and 100x Plan Apo VC oil immersion objective (NA 1.40). Image digitization was done with a Photometrics Evolve 512 EMCCD camera with a $1.2 \mathrm{x}$ postmagnification fitted in front of the camera. GFP was exited using $491 \mathrm{~nm}$ light generated by a Cobolt Calypso50 laser and the emitted light was bandpass filtered at 497-557 $\mathrm{nm}$. For FM4-64, $561 \mathrm{~nm}$ excitation light generated by a Cobolt Jive50 laser was used in combination with bandpass filtering at 570-620 nm. During image digitization a camera electron multiplication gain of 300 was employed and typical exposures were 1000-2000 ms for GFP and FM4-64. All components were operated by MetaMorph software.

FM4-64 was dissolved in $\mathrm{dH}_{2} \mathrm{O}$ at a final concentration of $10 \mu \mathrm{M}$ and cells were imaged 30-min after FM4-64 application.

\section{Time-gated confocal microscopy of infected $\boldsymbol{P}$. patens cells}

Live-cell imaging was performed on a Leica SP8X-SMD confocal microscope equipped with a hybrid single molecule detector ( $\mathrm{HyD})$ and an ultrashort pulsed white-light laser (WLL; 50\%; 1 ps at $40 \mathrm{MHz}$ frequency). The Leica Application Suite $\mathrm{X}$ was used as a software platform and imaging was conducted with a HC PL APO CS2 63x/1.20 water immersion objective, scan speed of $400 \mathrm{~Hz}$ and a resolution of $1024 \times 1024$ pixels and standard acquisition mode of the hybrid detector. GFP was excited with the $488 \mathrm{~nm}$ laser line (10\% laser output) of the WLL and the emitted light detected by HyD SMD 1 at 495$550 \mathrm{~nm}$ (gain 100). Autofluorescence of deposited material was excited with the $561 \mathrm{~nm}$ laser line (6\% laser output) of the WLL and emitted light was detected by HyD SMD 2 at 
568-600 nm (gain 100). To capture bright field images, PMT for transmittance was used as detector (gain 300). To remove autofluorescence in the GFP-channel (495-550 nm), timegating was activated on detector HyD SMD 1 at a gate reference wavelength of $488 \mathrm{~nm}$. For imaging, gate opening times were selected such that no autofluorescence was detectable in the GFP-channel. Specific opening times used are mentioned in the figures and figure legends. Time-gating was never activated for the red channel (568-550 nm). Images were captured with line and frame averages of one and four times, respectively. Z-series were collected with $0.356 \mu \mathrm{m}$ intervals. All image analysis was performed using ImageJ.

\section{Results}

\section{Time-gated confocal microscopy eliminates autofluorescence of papilla-like structures and hyphal encasements}

We first studied the localization of exocyst subunits Sec3, Sec5b, Sec5d and Sec6 in non-infected apical caulonemal cells, which are adventitious, fast-growing protonema cells with poorly developed chloroplasts (Menand et al., 2007). All exocyst subunits accumulated in transient foci associated with the plasma membrane (Supplementary Figure S1), confirming previous results (Tang et al., 2019). Next, the localization of exocyst subunits during different stages of pathogen infection was examined. We inoculated GFPtagged exocyst lines with zoospores of $P$. capsici and studied the infection process using confocal microscopy. In accordance with earlier work (Overdijk et al., 2016), we observed that papilla-like structures and deposited material around invasive hyphae strongly autofluoresce upon excitation at 561-nm (Figure 1A). The observed autofluorescent layer around invasive hyphae is reminiscent of an haustorial encasement, a term used to define the layer of wall-like material secreted by host cells to seal off fungal haustoria in non-host plants or mature haustoria in susceptible plants (Meyer et al., 2009; Wang et al., 2009; Lu et al., 2012; Faulkner, 2015). Consistent with the naming of haustorial encasements, we will refer to the autofluorescent structures around invasive hyphae as 'hyphal encasements'. The observed autofluorescence has a broad spectral range and is strongly present upon excitation with a $488 \mathrm{~nm}$ laser in the $495-550 \mathrm{~nm}$ emission range (Figure 1A). In our previous work, this autofluorescence in the GFP-channel was masked by high expression of LifeAct-GFP, a live cell actin probe (Overdijk et al., 2016). However, the GFP-tagged exocyst subunits have much lower expression levels and their fluorescence is masked by the strong autofluorescence of deposited material. To solve this problem, we have used time-gated confocal microscopy to eliminate autofluorescence from the GFP-channel (Figure 1B). This imaging technique uses the difference in fluorescence life time between autofluorescence and the fluorescence of GFP to eliminate autofluorescence (Kodama, 2016; Grossmann et al., 2018). The mean fluorescence life time of GFP is around $2.6 \mathrm{~ns}$ (Berezin \& Achilefu, 2010), while the fluorescence life time of autofluorescence, although variable and depending on the material, is generally much shorter (picosecond range) (Grossmann et al., 2018). Time-gated confocal microscopy was performed by exciting the GFP fluorophore using 
ultrashort pulses of laser light (1 picosecond) and the fluorescence was detected using a hybrid detector capable of monitoring a specific time-gate. Different time-gate settings were tested and in most cases, the autofluorescence of deposited material was eliminated when the time-gate was set between 0.5-24.5 ns (Figure 1A). Late infection stages with well-developed hyphal encasements did in some cases show autofluorescence when using the standard gating times of 0.5-24.5 ns, but this autofluorescence was abolished when the gate-opening time was decreased to 1-24.5 ns. It should be noted that in some cases Phytophthora hyphae still showed autofluorescence when using these settings, but that all autofluorescence inside the plant cell was eliminated. Therefore, we conclude that autofluorescence of deposited material at infection sites can be effectively eliminated by using time-gated confocal microscopy.
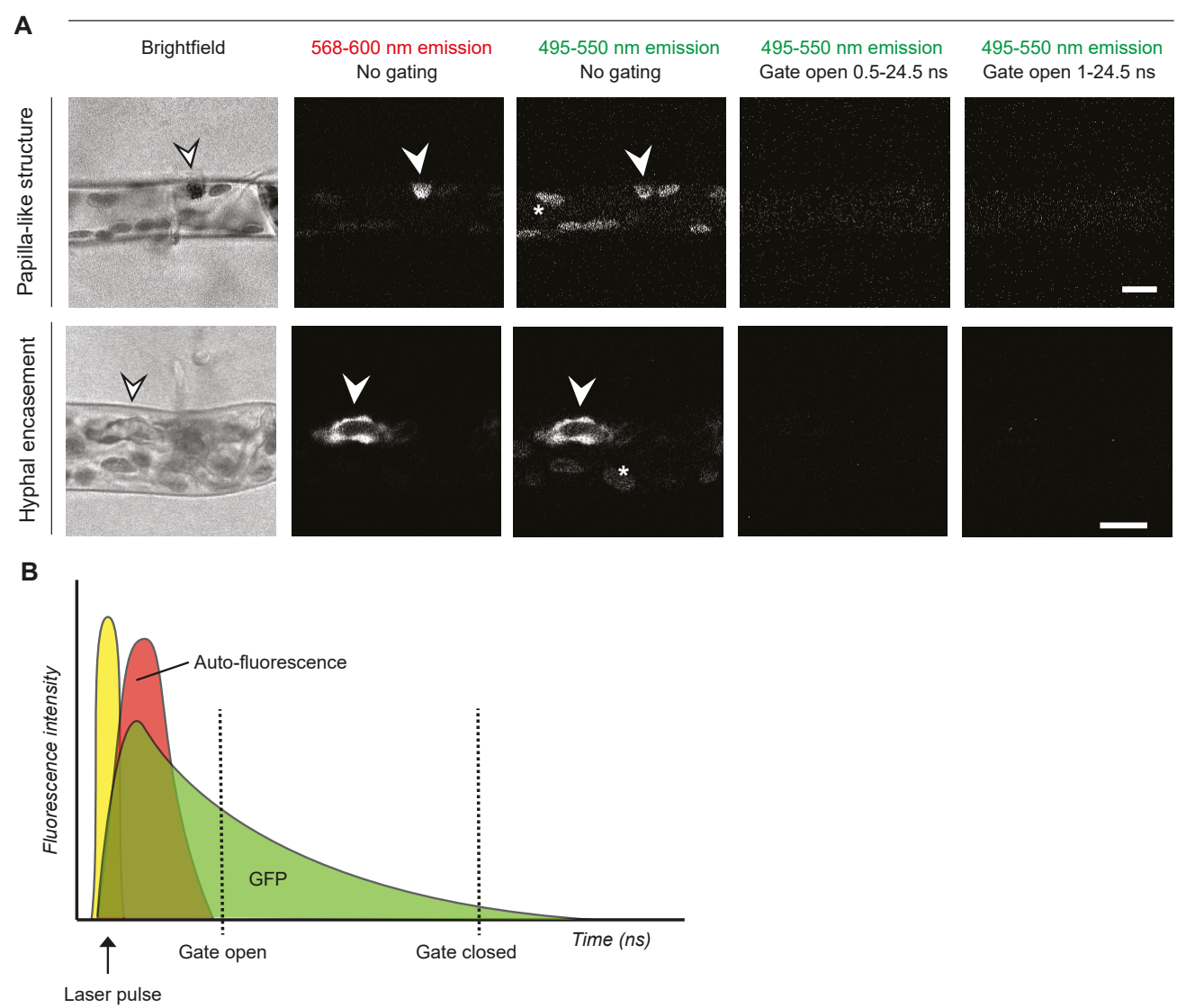

FIGURE 1. Time-gated fluorescence imaging eliminates autofluorescence of material deposited at infection sites. (A) Papilla-like structures and hyphal encasements around successfully penetrated $P$. capsici hyphae were imaged in wild type protonemal P.patens cells using two different time-gating settings for the green channel (495$550 \mathrm{~nm}$ emission). Arrowheads mark autofluorescence of a papilla-like structure and a hyphal encasement. Plastids are marked by stars. Autofluorescence in the red channel (568-600 nm emission) is used to visualize deposited material at infection sites. Imaging was done 3-6 hours after inoculation. Images are single confocal planes. Scale bars represent $10 \mu \mathrm{m}$. (B) Schematic representation of time-gated fluorescence imaging. Drawing not to scale. 


\section{Exocyst subunits are recruited to pathogen penetration sites before and during deposition of autofluorescent material}

We first focused on a very early infection stage, namely the moment that a Phytophthora germ tube encounters a moss cell and starts to form an appressorium. We observed that exocyst subunits Sec3a, Sec5b, Sec5d and Sec6 accumulate at the plasma membrane at the location where the Phytophthora hypha touches the cell (Figure 2). This is clearly different from the pattern in uninfected cells where we observed a punctate localization at the plasma membrane (Supplementary Figure S1). Sec3a, Sec5b, Sec5d and Sec6 were recruited to sites of attempted penetration before abundant accumulation of autofluorescent material was detected (Figure 2, arrowheads). During later infection stages, when more developed papilla-like structures could be detected, Sec3a, Sec5b, Sec5d and Sec6 accumulated on the plasma membrane surrounding the penetration attempt site (Figure 3). Fluorescence intensity plots reveal that they do not co-localize with the autofluorescent material of the papilla-like structures, but accumulate on the membrane around these structures (Figure 3B). The observed localization pattern around papilla-like structures is a prerequisite for a role of the exocyst in the targeted exocytosis required for their deposition.

\section{Exocyst subunits accumulate on the membrane surrounding invasive hyphae}

In some cases, the moss defensive responses are not sufficient to arrest penetration and invasive hyphae grow inside the moss cells, surrounded by an autofluorescent hyphal encasement (Overdijk et al., 2016). Since the moss cell is still alive, invasive hyphae are surrounded by an intact plant membrane. In line with the plant-derived membrane that is observed around Magnaporthe oryzae invasive hyphae (Kankanala et al., 2007), we call this membrane the 'extra-invasive hyphal membrane'. While the infection advances, the invasive hypha grows within the moss cell and the extra-invasive hyphal membrane needs to expand to accommodate the increased volume of the expanding invasive hypha. We questioned whether the exocyst localization reflects a role of the exocyst in facilitating the deposition of autofluorescent material for the hyphal encasements and the delivery of new extra-invasive hyphal membrane to accommodate intracellular hyphal growth. We indeed observed accumulation of the exocyst subunits Sec3, Sec5b, Sec5d and Sec6 on the extra-invasive hyphal membrane (Figure 4 and Figure 5). The exocyst subunits were not evenly distributed on the membrane surrounding the hyphal encasement, but preferentially accumulated on the extra-invasive hyphal membrane around hyphal tips (Figure 4 and Figure 5, arrowheads). These hyphal tips did not show the characteristic autofluorescent encasing (Figure 5, arrowheads). The observed exocyst subunit localization pattern suggests that the exocyst also plays a role in facilitating the expansion of the extra-invasive hyphal membrane and the deposition of defence materials at this location. 


\section{Chapter 4}

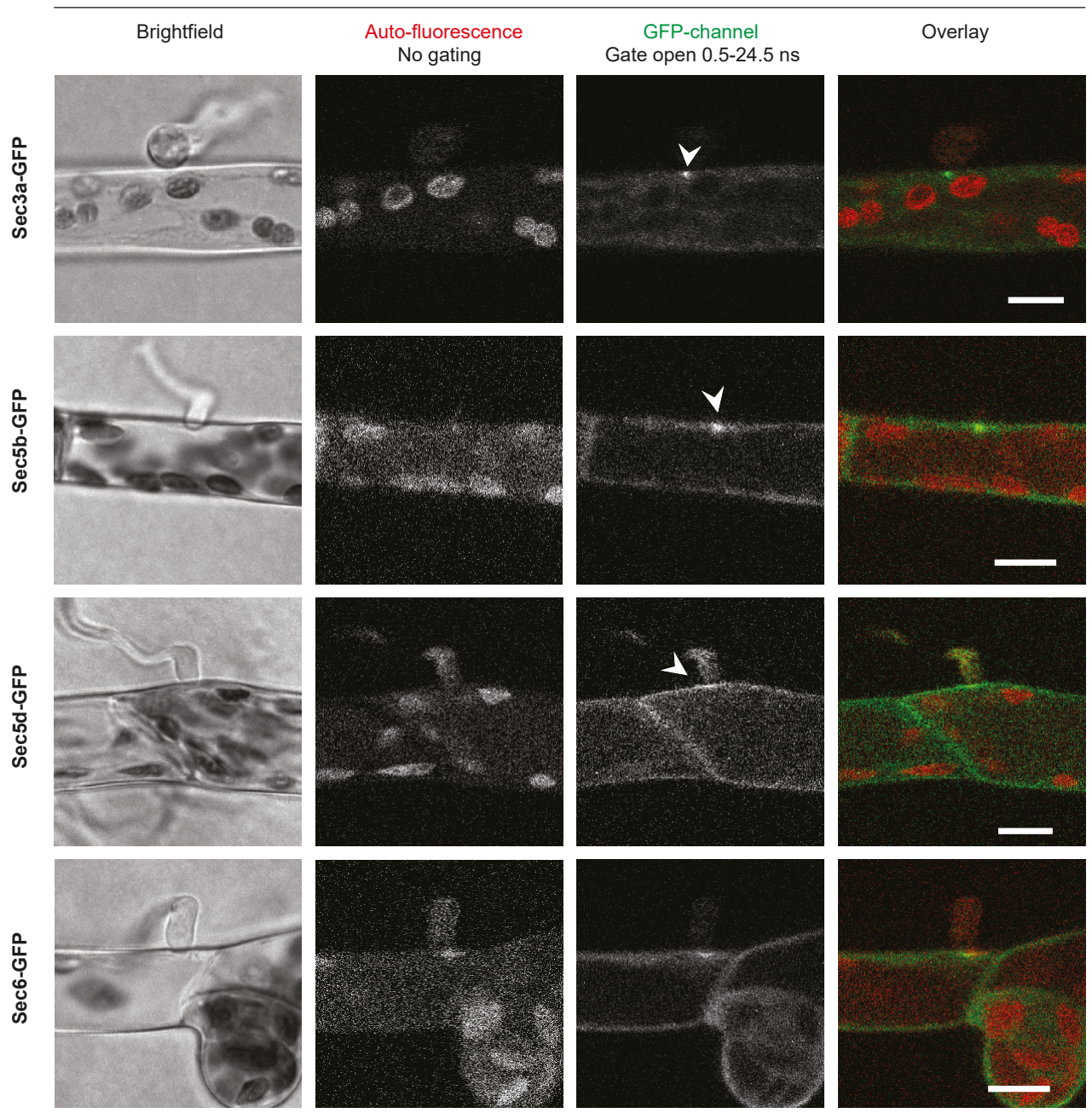

FIGURE 2. Exocyst subunits accumulate at the plasma membrane at attempted penetration sites. Localization of P. patens exocyst subunits Sec $3 a$, Sec5b, Sec5d and Sec6 visualised in P. capsici infected protonemal cells expressing a GFP-tagged version of the indicated exocyst subunit. Autofluorescence in the red channel is used to mark deposited material upon pathogen attack. Arrowheads mark exocyst subunit localization at attempted penetration sites where no abundant deposition of autofluorescent material has occurred. Imaging was done 3-6 hours after inoculation. Images are maximum z-projections of 4 confocal planes spaced $0.36 \mu \mathrm{m}$ apart, except for the image of Sec3a which is a single focal plane. Scale bars represent $10 \mu \mathrm{m}$. N: nucleus. 

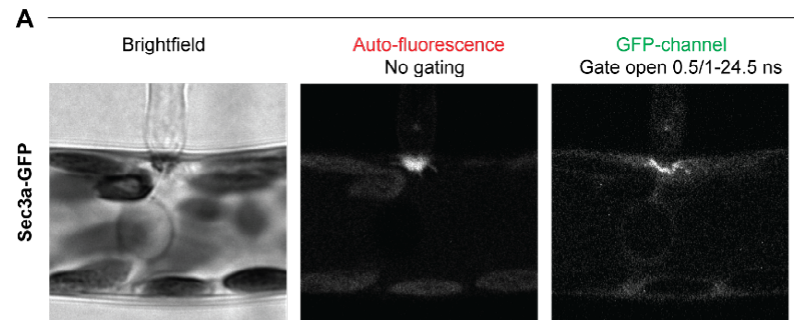

Overlay
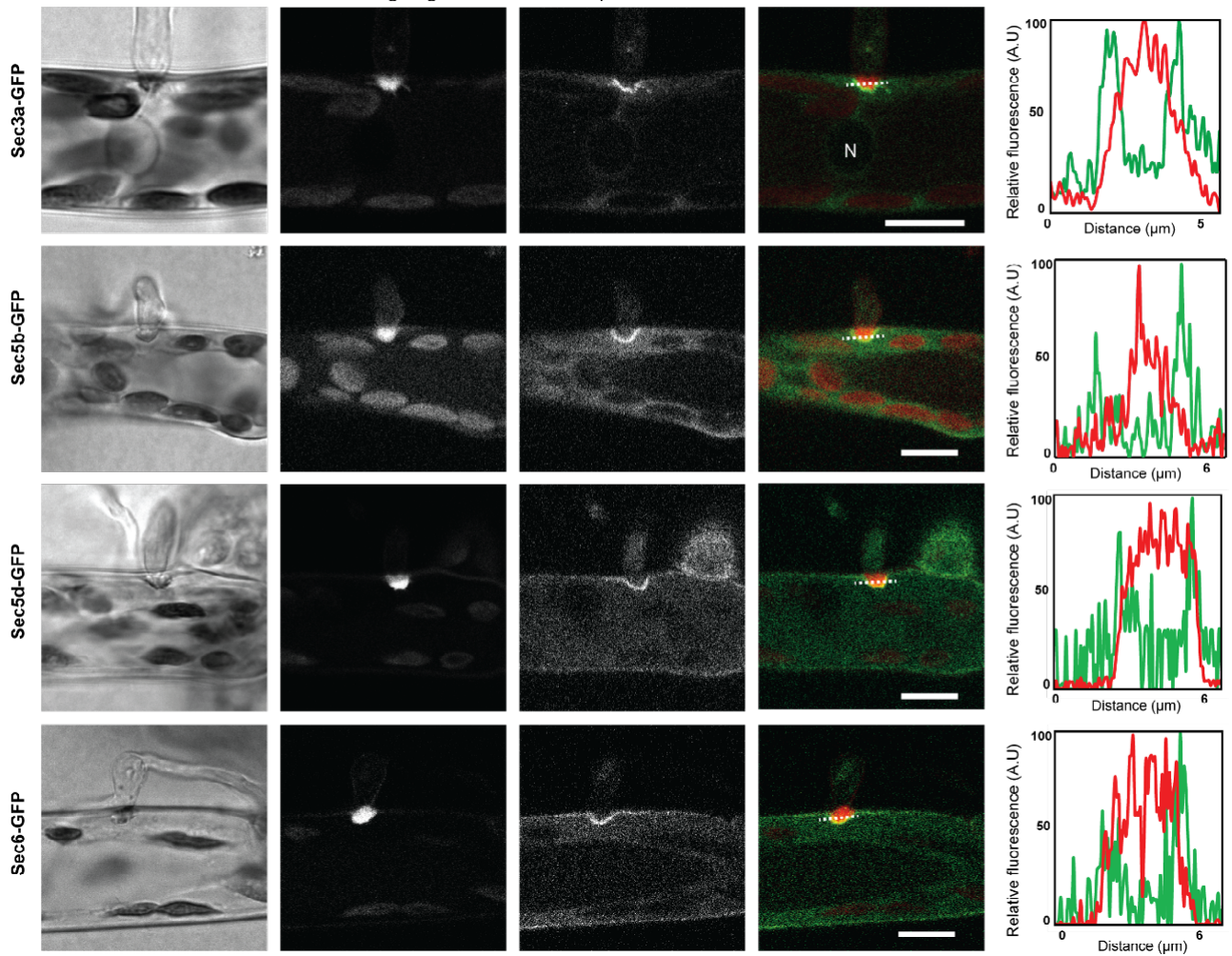

FIGURE 3. Exocyst subunits accumulate at the plasma membrane surrounding papilla-like structures. (A) Localization of $P$. patens exocyst subunits Sec3a, Sec5b, Sec5d and Sec6 visualized in P. capsici infected protonemal cells expressing a GFP-tagged version of the indicated exocyst subunit. Autofluorescence in the red channel is used to mark deposited material of papilla-like structures. Imaging was done 3-6 hours after inoculation. Time-gating is 1-24.5 ns for Sec5b and Sec6 and 0.5-24.5 ns for Sec3a and Sec5d. Images of Sec3a and Sec5d are single confocal planes and images of Sec5b and Sec6 are maximum z-projections of $1.4 \mu \mathrm{m}$. Scale bars represent $10 \mu \mathrm{m}$. N: nucleus. (B) Relative fluorescence intensity along the dotted lines in A. Green line: exocyst-GFP, red line: autofluorescence. 


\section{Chapter 4}

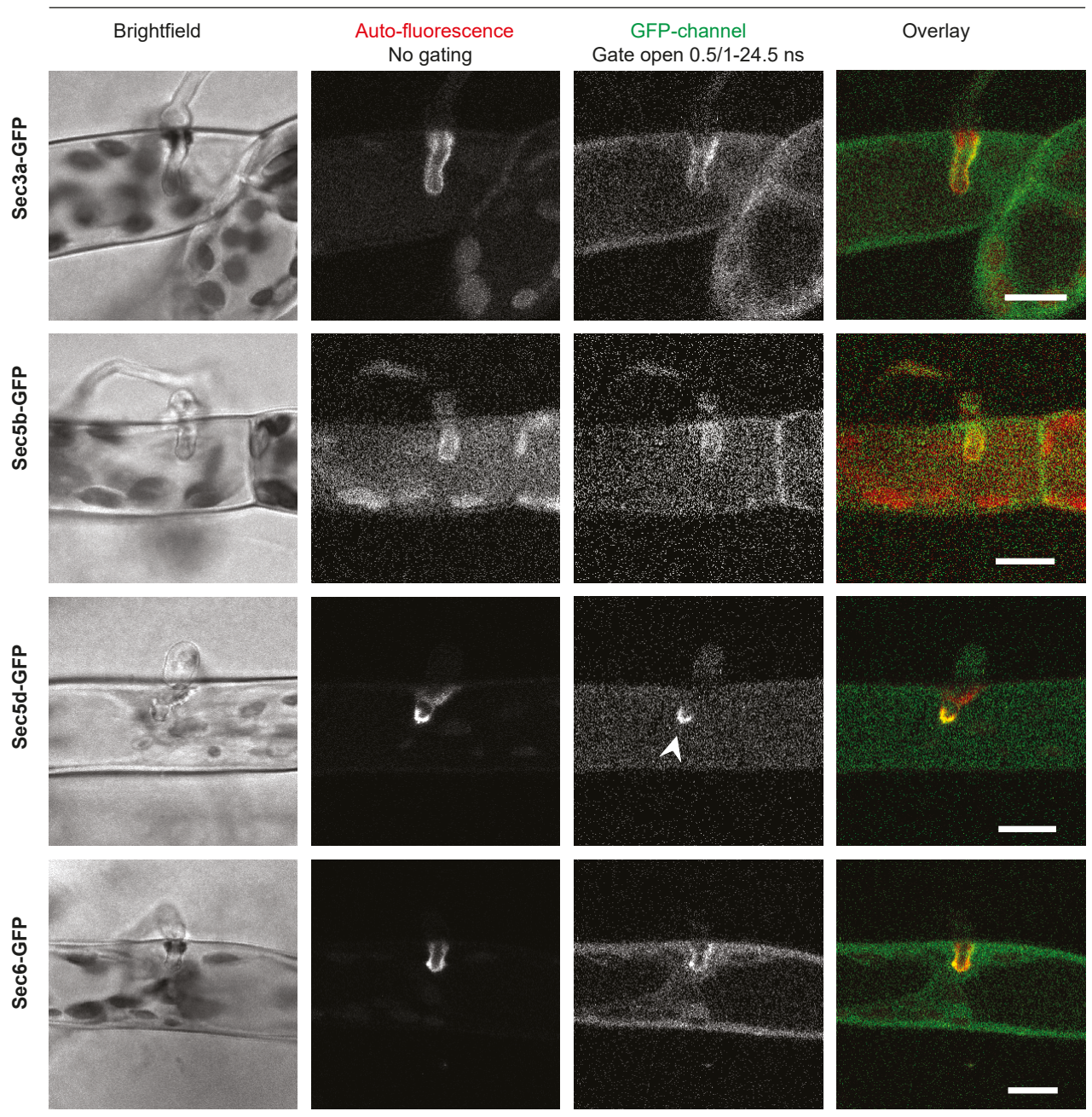

FIGURE 4. Invasive hyphae are surrounded by exocyst subunits. Localization of $P$. patens exocyst subunits Sec3a, Sec5b, Sec5d and Sec6 visualized in P. capsici infected protonemal cells expressing a GFP-tagged version of the indicated exocyst subunit. Autofluorescence in the red channel is used to mark deposited material in hyphal encasements. Arrowheads mark the site where exocyst subunit signal is seen at the tip of invasive hyphae. Imaging was done 3-6 hours after inoculation. Time-gating is 1-24 ns for all subunits, except for Sec6 were 0.5-24.5 ns was used. Images for Sec3a, Sec5b and Sec5d are maximum z-projections of $2.5 \mu \mathrm{m}$ while the image of Sec6 is a maximum z-projection of $1.4 \mu \mathrm{m}$. Scale bars represent $10 \mu \mathrm{m}$. 


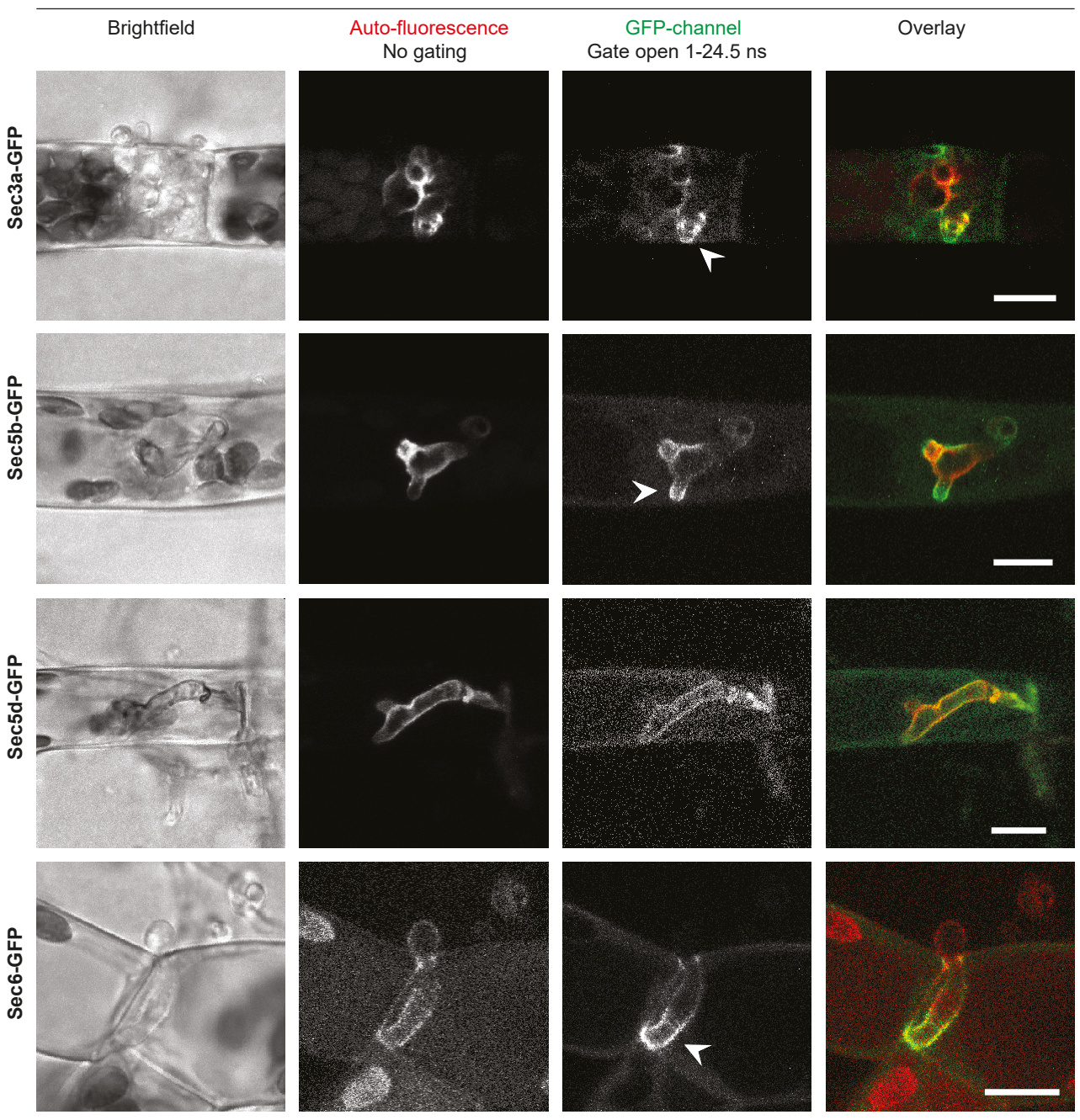

FIGURE 5. At a late infection stage hyphal encasements are surrounded by exocyst subunits. Localization of $P$. patens exocyst subunits Sec3a, Sec5b, Sec5d and Sec 6 visualized in P. capsici infected protonemal cells expressing a GFP-tagged version of the indicated exocyst subunit. Autofluorescence in the red channel is used to mark deposited material around invasive hyphae. Arrowheads mark sites where exocyst subunit signal is seen at the tip of invasive hyphae. Imaging was done 3-6 hours after inoculation. Image of Sec3a is an average z-projection of $7.1 \mu \mathrm{m}$ and images of Sec5b, Sec5d and Sec6 are maximum z-projections of 3.2, 1.8 and $1.4 \mu \mathrm{m}$, respectively. Scale bars represent $10 \mu \mathrm{m}$. 


\section{Vamp72A1-labelled exocytotic vesicles accumulate around papilla-like structures and hyphal encasements}

The exocyst tethers vesicles, in this case presumably defence-related vesicles, to the cell membrane (Martin-Urdiroz et al., 2016). To investigate whether these vesicles indeed accumulate at infection sites in $P$. patens, we selected a marker for defence-related exocytotic vesicles in $P$. patens based on studies in A. thaliana. In this plant species it was found that two members of the v-SNARE Vamp72-family, namely Vamp721 and Vamp722, accumulate around powdery-mildew penetration sites (Kwon et al., 2008). The exocyst has been hypothesized to tether Vamp721-coated vesicles to the plasma membrane, since the density of GFP-Vamp721 foci at the plasma membrane was reduced in A. thaliana Exo70A1 mutant plants (Fendrych et al., 2013). The $P$. patens genome contains five genes encoding proteins of the Vamp72 family (Sanderfoot, 2007) and the closest homolog to the A. thaliana Vamp72 family was selected as a defence-related vesicle marker, namely PpVamp72A1 (Supplementary Figure S2), which is known to label secretory vesicles in moss (Furt et al., 2013; Bibeau et al., 2017). We integrated a GFP-encoding DNA fragment upstream of the Vamp72A1 coding region, resulting in a moss line with endogenous expression levels of GFP-labelled Vamp72A1 (Supplementary Figure S3). In those lines, we observed GFP-Vamp72A1 fluorescence on the plasma-membrane and in cellular compartments (Supplementary Figure S3). Staining with the endocytic dye FM4-64 showed that these compartments were endosomal compartments.

Upon Phytophthora infection, Vamp72A1 accumulated around papilla-like structures and hyphal encasements (Figure 6). Similar to the exocyst subunits, GFP-Vamp72A1 initial accumulation around a penetration attempt site occurred before autofluorescent materials could be detected (Figure 6, arrowhead). However, comparison of the fluorescence intensity plots in Figure 3 and Figure 6, revealed that unlike the exocyst subunits, Vamp72 signal not only associates with the cell membrane, but is also present in the cytoplasm that surrounds the infection site, suggesting that vesicles containing defence materials accumulate in the cytoplasm around infection sites. Besides the localization of Vamp72A1 in the cytoplasm around papilla-like structures, GFP-Vamp72A1 signal was also observed within some papilla-like structures (Figure 6, arrow). 


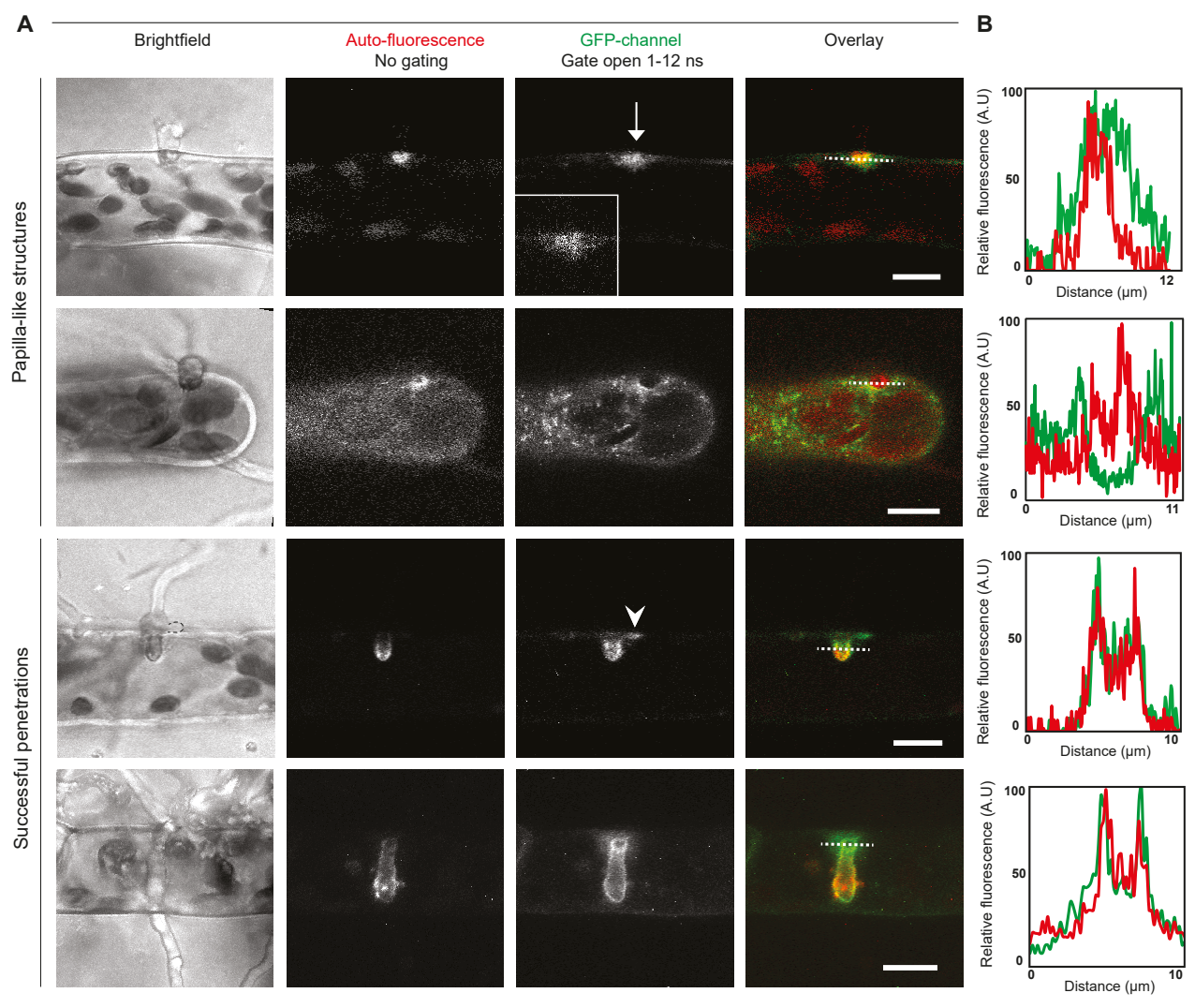

FIGURE 6. Vamp72A1-marked vesicles accumulate in the cytoplasm surrounding papilla-like structures and hyphal encasements. (A) Localization of P. patens GFP-Vamp72A1 visualized in P. capsici infected protonemal cells. Autofluorescence in the red channel is used to mark deposited material at infection sites. Arrow marks GFP signal within papilla-like structure and arrowhead marks GFP-Vamp7A1 localization at an attempted penetration site where no autofluorescent material is deposited. Imaging was done 3-6 hours after inoculation. Image of papilla-like structure in the first row is an average z-projection of $7.5 \mu \mathrm{m}$; insert represents a single confocal plane. Image of the papilla-like structure in the second row is a maximum z-projection of $2.5 \mu \mathrm{m}$ from a top view. Image of the successful penetration in the third row is an average z-projection of $3 \mu \mathrm{m}$ and in the fourth row a maximum z-projection of $28 \mu \mathrm{m}$. Scale bars represent $10 \mu \mathrm{m}$. (B) Relative fluorescence intensity along the dotted lines in A. Green line: GFP-Vamp72A1, red line: autofluorescence. 


\section{Discussion}

Time-gated confocal microscopy is an appropriate imaging tool to eliminate undesired autofluorescence based on the difference of fluorescence life times of a fluorophore and autofluorescence (Kodama, 2016; Grossmann et al., 2018). This is particularly useful in cases where (parts of) plant cells show high levels of autofluorescence, for example upon wounding or upon pathogen attack when producing phytoalexins or papillae, or during secondary cell wall deposition. In this study, we demonstrated the high potential of timegated microscopy in solving imaging problems in infected plant cells and for this we exploited the recently described Physcomitrella-Phytophthora pathosystem (Overdijk et al., 2016). We focussed on the localization of exocyst subunits Sec3a, Sec5b, Sec5d and Sec6 and showed that they accumulate around sites where a Phytophthora hyphae touches the cell (penetration attempt site) and, later during infection, around papilla-like structures and invasive hyphae. We also used the v-SNARE Vamp72A1 to mark defence-related vesicles in $P$. patens and showed that these vesicles accumulate in the cytoplasm around infection sites.

The observed polarized accumulation of exocyst subunits at infection sites suggests that the exocyst is directly involved in facilitating polarized exocytosis of vesicles containing defence-related autofluorescent material. This localization resembles polar accumulation of exocyst subunits in other polarized secretion processes. For example, during tip growth A. thaliana Sec3a accumulates at growing tips of pollen tubes where cell wall material is deposited (Bloch et al., 2016) and during xylem formation Sec8 localizes at secondary cell wall thickenings (Vukasinovic \& Zarsky, 2016). The accumulation of exocyst subunits at penetration attempt sites and around papilla-like structures, is consistent with the suggested role for the exocyst in papillae formation and callose deposition (Pecenkova et al., 2011; Kulich et al., 2015; Du et al., 2018a; Guo et al., 2018b; Kulich et al., 2018).

In plants encountering beneficial microbes exocyst subunits are targeted to sites of polarized exocytosis, e.g. in Medicago truncatula exocyst subunits Exo70i and Exo84b localize to tips of arbuscular branches and, in addition, Exo70i is recruited to tips of growing infection threads to facilitate rhizobium symbiosis (Genre et al., 2012; Zhang et al., 2015; Gavrin et al., 2017). During both, beneficial and pathogenic interactions a membrane-based interface is formed for which the delivery of new membranes by exocytosis is essential. In the Physcomitrella-Phytophthora system, we show that exocyst subunits accumulate at the extra-invasive hyphal membrane, which is consistent with the localization of exocyst subunits during symbiotic interactions. This suggests that pathogens hijack the membrane trafficking machinery that operates during beneficial interactions to accommodate invasive hyphal growth (Leborgne-Castel \& Bouhidel, 2014; Yamazaki \& Hayashi, 2015). The question remains whether exocyst subunits also accumulate at the extrahaustorial membrane. In P. patens infected with Phytophthora no obvious haustoria are formed (Overdijk et al., 2016). However, in N. benthamiana cells infected with $P$. infestans there is an association of endosome markers with the extrahaustorial membrane, suggesting that this membrane is also derived by re-routing of plant endomembrane trafficking (Bozkurt et al., 2015). 
Studies in A. thaliana and Medicago truncatula have shown that v-SNARE Vamp721 is localized at sites of polarized exocytosis during both beneficial and pathogenic plantmicrobe interactions (Kwon et al., 2008; Ivanov et al., 2012). Mosses like P. patens diverged around 450 million years ago from a common ancestor shared with seed plants (Knight et al., 2009), and hence the similar Vamp72 localization pattern in moss and seed plants points to a conserved role of Vamp72-vesicles in plant-microbe interactions.

In budding yeast, six of the eight subunits, i.e. Sec5, Sec6, Sec8, Sec10, Sec15 and Exo84, arrive at polarized membrane domains on vesicles via transport on actin cables (Boyd et al., 2004). In plants, none of the exocyst subunits appear to associate with a detectable cytoplasmic population of exocytotic vesicles (Fendrych et al., 2013; Zhang et al., 2013; Bloch et al., 2016; Sekeres et al., 2017; Tang et al., 2019). Also from our work it is clear that the localization pattern of exocyst subunits and Vamp72A1-labeled vesicles differs: unlike Vamp72A1, exocyst subunit accumulation in the cytoplasm around infection sites was less prominent. It thus appears that exocyst subunits Sec3a, Sec5b, Sec5d and Sec6 are not consistently associated with Vamp72A1-labeled vesicles. However, Vamp721 vesicles in $A$. thaliana rely on the exocyst subunit Exo70A1 for recruitment to the plasma membrane (Fendrych et al., 2013). Assuming a similar reliance in P. patens, association of exocyst subunits to Vamp72-labelled vesicles is likely to occur in close proximity with the plasma membrane and not during vesicle transport.

Besides the intracellular Vamp72A1 localization, we also observed Vamp72A1-GFP within papilla-like structures outside the plasma membrane. A similar phenomenon has been observed in seed plants. For example, PMR4, PEN1/SYP121, SNAP33, PEN3 and RPW8.2, were found to accumulate extracellularly within papillae in response to pathogen attack (Meyer et al., 2009; Lu et al., 2012). A possible explanation for this extracellular fluorescence is the direct fusion of multivesicular bodies (MVBs) with the plasma membrane and subsequent release of extracellular vesicles or exosomes inside papilla (An et al., 2006; Wang et al., 2017). Two types of unconventional exocytosis could act as sources for MVBs, autophagy and exocyst-positive organelles (EXPOs) (Gu et al., 2017). The exocyst, or more specifically the sub-complex consisting of exocyst subunits Exo70B1, Sec5 and Exo84, functions in autophagy-related transport to the vacuole (Kulich et al., 2013). Therefore, it is hypothesized that the exocyst might be involved in unconventional exocytosis via autophagy-related processes (Pecenkova et al., 2017). In N. benthamiana plants infected with $P$. infestans, it has been shown that autophagosomes are diverted towards the extrahaustorial membrane and that they are important for polarized defence responses (Dagdas et al., 2018). This suggests that, besides a role in targeting conventional exocytosis to an infection site, the exocyst could be involved in defence-related exocytosis by facilitating exocytosis of autophagy- or EXPO-related MVBs.

Our observations raise the question what could trigger the re-localization of exocyst subunits to infection sites. It is likely that this is initiated by the physical encounter with the pathogen itself and/or molecular cues (e.g. pathogen associated molecular patterns, PAMPs) produced by the pathogen. Hardham et al. (2008), observed that the pressure of a microneedle 


\section{Chapter 4}

touching a cell is sufficient to induce rapid local fine filamentous actin accumulation. Since fine filamentous actin reduces Golgi body motility (Akkerman et al., 2011), the actin accumulation might trap vesicles near the infection site. Moreover, treatment with PAMPs like the fungal chitin and the bacterial flg22, results in changes in actin abundance (HentyRidilla et al., 2013), which might facilitate transport to infection sites.

The next question then is how the exocyst subunits are recruited towards sites of attempted penetration. From yeast it is known that exocyst localization at the plasma membrane is mediated by the interaction of Sec3 with phosphatidylinositol-4,5-bisphosphate $\left(\mathrm{PIP}_{2}\right.$ ) and with small GTPases (Mei \& Guo, 2018). A. thaliana Sec3a also interacts with $\mathrm{PIP}_{2^{\prime}}$, however this interaction does not seem to be required for the polar localization of Sec3a in pollen tubes (Bloch et al., 2016; Li, Y et al., 2017). Apparently in plants, unlike in yeast, the interaction with $\mathrm{PIP}_{2}$ is not driving the recruitment of the exocyst to sites of polarized exocytosis. Regarding small GTPases, two exocyst subunits have been found to indirectly interact with the small GTPase-family Rho of Plants (ROPs) via ROP effectors acting downstream of ROPs (Lavy et al., 2007; Hong et al., 2016). Firstly, ICR1, an interactor of activated ROPs, interacts with $A$. thaliana Sec3a and secondly, RIC7, a ROP2 interacting protein, interacts with $A$. thaliana Exo70B1 (Lavy et al., 2007; Hong et al., 2016). This kind of interactions may point to an (indirect) role for small GTPases in recruiting the exocyst in plant cells to infection sites.

In summary, using time-gated confocal microscopy we were able to show that exocyst subunits are re-directed towards infection sites and this strengthens the idea that exocystmediated vesicle tethering is required for exocytosis of defence-related vesicles. We show the value of time-gated confocal microscopy for visualizing proteins in plant cells challenged by and interacting with pathogens. The advantages of time-gated microscopy should be exploited to further unravel the molecular and cellular processes underlying plant defence, thereby providing insights instrumental for breeding durable resistant plants. 


\section{Acknowledgements}

We kindly thank Jeroen de Keijzer (Wageningen University, The Netherlands) for generating and sharing the peGFP-Nter vector and Wouter van Bakel (Wageningen University, The Netherlands) for technical assistance. Time-gated imaging was performed on a confocal microscope supported by a NWO Middelgroot Investment Grant (721.011.004; JWB). This research was supported by the Earth and Life Sciences Division of The Netherlands Organization for Scientific Research (NWO; PhD grant to EO).

\section{Supplemental data}

A

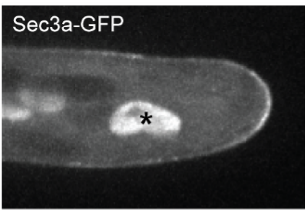

B

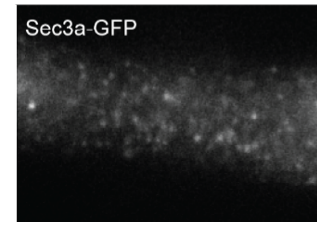

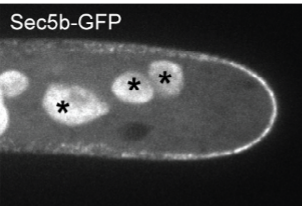

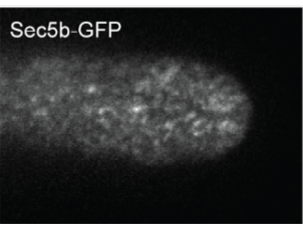

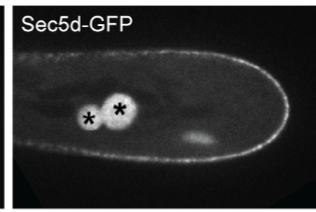
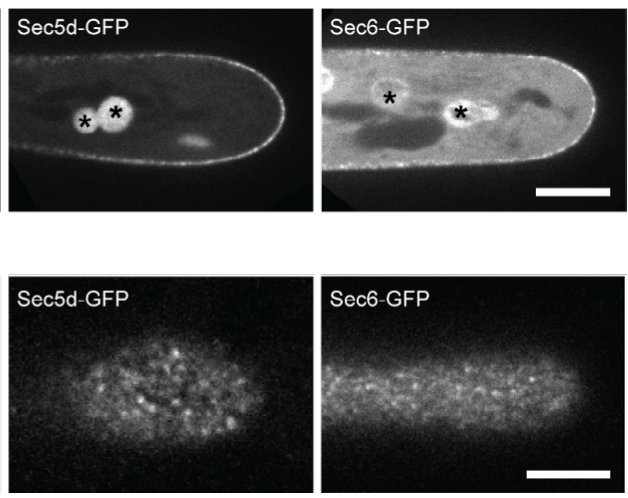

SUPPLEMENTARY FIGURE S1. P. patens exocyst subunits Sec3a, Sec5b, Sec5d and Sec6 have a punctate localization on the plasma membrane of protonemal cells. (A) Localization of $P$. patens exocyst subunits Sec3a, Sec5b, Sec5d and Sec6 visualized in non-infected caulonemal apical cells expressing a GFP-tagged version of the indicated exocyst subunit at a median confocal plane. Plastids are marked by stars. Images are maximum z-projections $(1.5 \mu \mathrm{m})$ and scale bars represent $10 \mu \mathrm{m}$. (B) Cortical localization of exocyst subunits Sec3a, Sec5b, Sec5d and Sec6 visualised in non-infected caulonemal apical cells expressing a GFP-tagged version of the indicated exocyst subunit at a single confocal plane. Scale bars represent $10 \mu \mathrm{m}$. 
A

\begin{tabular}{ccccc} 
Name & v1.6 identifier & v3.3 identifier & $\begin{array}{c}\text { \% identity with } \\
\text { AtVamp721 }\end{array}$ & $\begin{array}{c}\text { \% identity with } \\
\text { AtVamp722 }\end{array}$ \\
\hline Vamp72A1 & Pp1s219_102V6 & Pp3c4_13580V3.1 & 72.10 & 70.90 \\
\hline Vamp72A2 & Pp1s391_69V6 & Pp3c12_11090V3.1 & 71.70 & 70.50 \\
\hline Vamp72B1 & Pp1s226_119V6 & Pp3c2_2310V3.1 & 54.80 & 53.30 \\
\hline Vamp72B2 & Pp1s269_22V6 & Pp3c14_26140V3.1 & 58.00 & 56.40 \\
\hline Vamp72B3 & Pp1s68_120V6 & Pp3c17_22240V3.1 & 54.30 & 53.30 \\
\hline
\end{tabular}

B

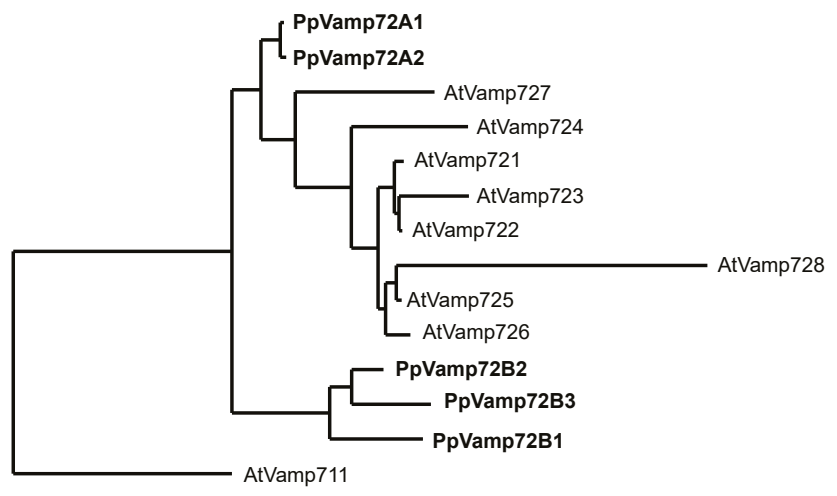

0.4

C

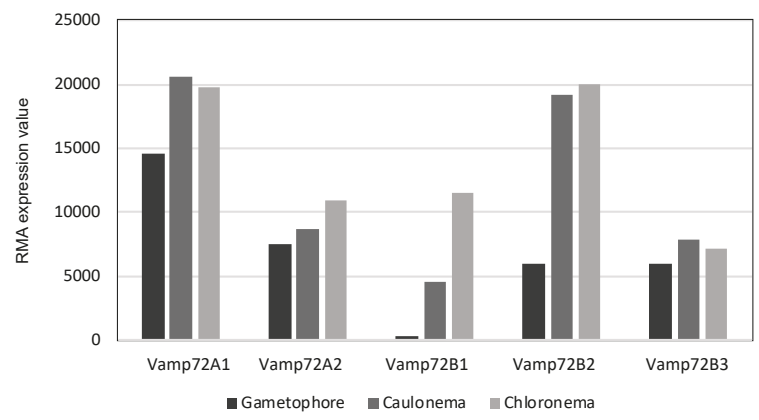

D
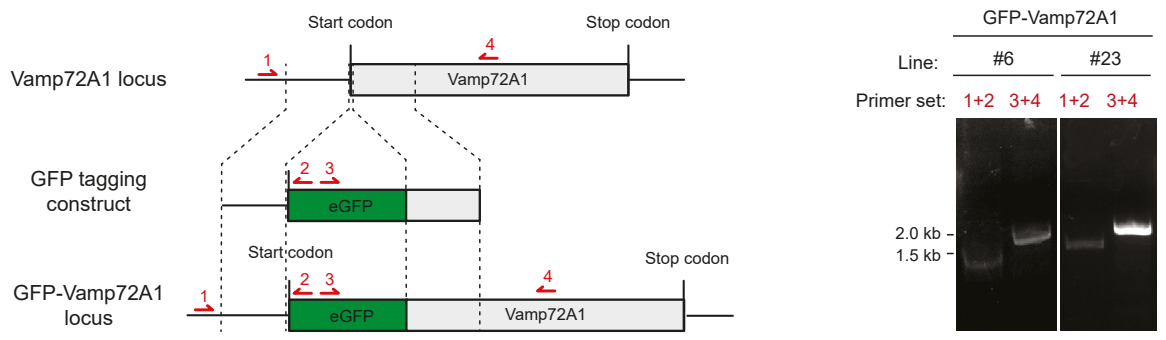

$1+2: 1456$ bp $\quad 3+4: 1866$ bp

SUPPLEMENTARY FIGURE S2. $P$. patens Vamp72-family analysis and generation and verification of $P$. patens GFP-Vamp72A 1 lines (legend continues on next page). 
SUPPLEMENTARY FIGURE S2. $P$. patens Vamp72-family analysis and generation and verification of $P$. patens GFP-Vamp72A1 lines. (A) Table listing P. patens Vamp72-family proteins. The locus identifiers for two versions of the $P$. patens genome assembly are given as well as their percentage identity with $A$. thaliana Vamp721/722 at the protein level. (B) Phylogenetic tree of Vamp721 proteins of $P$. patens and $A$. thaliana using the maximum likelihood method. A. thaliana Vamp711 served as an outgroup. Tree made with Phylogeny.fr. Horizontal branch length represents the number of amino acid substitutions. (C) Expression analysis of P. patens Vamp72 genes. The robust multi-array average (RMA) expression value is plotted for three different moss tissues. Expression data was obtained from the publically available Physcomitrella transcriptome atlas (Ortiz-Ramirez et al. 2016). (D) Schematic representation of the genomic locus in P. patens containing the Vamp72 encoding gene (grey box) and construct used for N-terminal GFP-tagging via homologous recombination (dashed lines). In fluorescently labelled moss lines, the original start codon is replaced with a fragment containing an eGFP encoding sequence (green box). Red arrows denote primer binding sites used for confirmation of the obtained lines by PCR analysis. For each indicated moss line with a knocked-in construct for fluorescent labelling, the product obtained after $P C R$ reaction and its predicted size are given. The numbers of two independent transformants are given above the gel images.

A

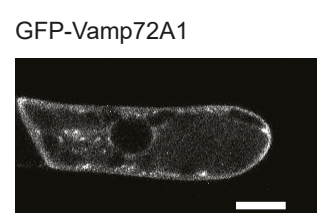

B

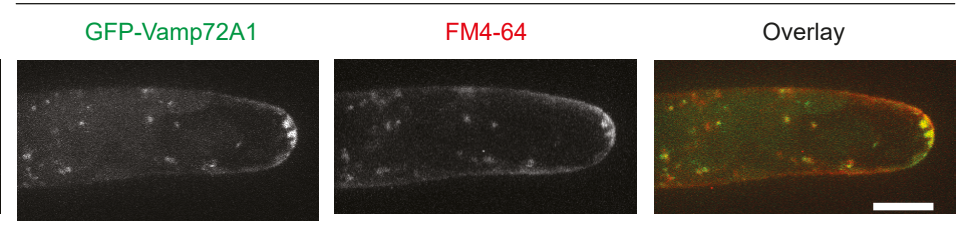

SUPPLEMENTARY FIGURE S3. Vamp72A1 localization in non-infected $\boldsymbol{P}$. patens cells. Localization of GFPVamp72A1 visualized in non-infected caulonemal apical cell stained with FM4-64 membrane dye. A is an average z-projection of $3.5 \mu \mathrm{m}$ and made with time-gating of 1-12 ns. B is a maximum z-projection of $13 \mu \mathrm{m}$ and no timegating. Scale bars represent $10 \mu \mathrm{m}$.

SUPPLEMENTARY TABLE S1. Primers used in this study.

\begin{tabular}{|c|c|c|c|}
\hline Primer & Use & Sequence $\left(5^{\prime}>3^{\prime}\right)$ & RE site \\
\hline JK91 & Cloning of peGFP-Nter vector & AGCTTGATATCGGGCGCGCCGCTAGCG & - \\
\hline JK92 & Cloning of peGFP-Nter vector & AATTCGCTAGCGGCGCGCCCGATATCA & - \\
\hline EO127 & Cloning Vamp72A1 left targeting arm & AAAGGGCCCTTGGGAAGGGAGTTTAATGTGG & Apal \\
\hline EO128 & Cloning Vamp72A1 left targeting arm & AAAATCGATCTTTACAACGGTGGGTGTCCCC & Clal \\
\hline EO129 & Cloning Vamp72A1 right targeting arm & AAAGGCGCGCCGGTACTCAGTCGCTGATTTACA & Ascl \\
\hline EO130 & Cloning Vamp72A1 right targeting arm & AAAGCGGCCGCACAGCTAATCGTCCCAACAATG & Notl \\
\hline EO153 & Genotyping Vamp72A1 locus & CGATACGAGCCCTTGCCTTCTTC & - \\
\hline E0154 & Genotyping Vamp72A1 locus & ACTTCACGGGAAAGGTCCAGC & - \\
\hline $\mathrm{JK} 73$ & General forward primer on GFP & AAGCCATTGGTGAGCAAGGGCGAGGAGC & - \\
\hline JK112 & General reverse primer on GFP & GCCGGACACGCTGAACTTGTG & - \\
\hline JK114 & General forward primer on resistance cassette & ACTCAAGAGGATAAAACCTCACC & - \\
\hline
\end{tabular}


SUPPLEMENTARY TABLE S2. Plasmids used in this study.

\begin{tabular}{llc}
\hline Plasmid & Usage & Source \\
\hline peGFP-Nter & Vector for constructing N-terminal GFP fusion proteins, no antibiotic resistance & This study \\
\hline GFP-Vamp72A1 & N-terminal GFP tagging of Vamp72A1 & This study \\
\hline
\end{tabular}

SUPPLEMENTARY TABLE S3. $P$. patens strains used in this study.

\begin{tabular}{|c|c|c|c|c|}
\hline Name & Use & Selected clone & Background strain & Reference \\
\hline Sec3a-GFP & $\begin{array}{l}\text { Sec3a visualization during } \\
\text { pathogen attack }\end{array}$ & $\# 15$ & $\begin{array}{l}\text { Wild-type isolate } \\
\text { Gransden }\end{array}$ & Tang et al., 2019 \\
\hline Sec5b-GFP & $\begin{array}{l}\text { Sec5b visualization during } \\
\text { pathogen attack }\end{array}$ & $\# 42$ & $\begin{array}{l}\text { Wild-type isolate } \\
\text { Gransden }\end{array}$ & Tang et al., 2019 \\
\hline Sec5d-GFP & $\begin{array}{l}\text { Sec5d visualization during } \\
\text { pathogen attack }\end{array}$ & $\# 39$ & $\begin{array}{l}\text { Wild-type isolate } \\
\text { Gransden }\end{array}$ & Tang et al., 2019 \\
\hline Sec6-GFP & $\begin{array}{l}\text { Sec6 visualization during } \\
\text { pathogen attack }\end{array}$ & $\# 37$ & $\begin{array}{l}\text { Wild-type isolate } \\
\text { Gransden }\end{array}$ & Tang et al., 2019 \\
\hline GFP-Vamp72A1 & $\begin{array}{l}\text { Exocytotic vesicle visualization } \\
\text { during pathogen attack }\end{array}$ & \#6 & $\begin{array}{l}\text { Wild-type isolate } \\
\text { Gransden }\end{array}$ & This study \\
\hline
\end{tabular}




\section{Chapter 5}

Phytophthora infestans RXLR effector AVR1 disturbs the growth of Physcomitrella patens, without affecting Sec5 localization

Elysa J.R. Overdijk
Vera Putker
Joep Smits
Han Tang

Klaas Bouwmeester

Francine Govers

Tijs Ketelaar 


\section{Abstract}

The RXLR effector AVR1 from the oomycete pathogen Phytophthora infestans suppresses host defence by targeting Sec5. Sec5 is a subunit of the exocyst, a protein complex that is important for mediating polarized exocytosis during plant development and defence against pathogens. So far, the mechanism by which AVR1 manipulates Sec5 functioning in plants is unknown. In this study, we used the moss Physcomitrella patens to analyse the effect of AVR1 on Sec5 localization and functioning. Yeast-two-hybrid assays showed that AVR1 interacts with two out of four P. patens Sec5 homologs, i.e. PpSec5b and PpSec5d, whereas AVR1 $1^{\Delta T}$, a truncated version of AVR1, did not interact. When $P$. patens lines carrying $\beta$-estradiol inducible $A V R 1$ or $A V R 1^{\Delta T}$ transgenes were triggered to express $A V R 1$ or $A V R 1^{\Delta T}$, they showed defects in the development of caulonemal protonema cells and abnormal cell morphologies in chloronema cells. Similar phenotypes were observed in Sec5-or Sec6-silenced P. patens lines, suggesting that both AVR1 and AVR1 ${ }^{\Delta T}$ affect exocyst functioning in $P$. patens. The presence of AVR1 however, did not seem to alter Sec5 localization. In $\beta$-estradiol-treated and untreated transgenic AVR1 lines, the Sec5 localization at the plasma membrane in growing caulonema cells and during pathogen attack was comparable. These findings suggest that AVR1 impairs Sec5 functioning, without altering its subcellular localization. 


\section{Introduction}

Phytophthora species are notorious oomycete plant pathogens that cause disease on a wide range of host plants (Kroon et al., 2012). One of the best studied species is Phytophthora infestans, the causal agent of late blight that contributes to great losses in potato and tomato production worldwide. P. infestans promotes host colonization by secreting effectors that suppress plant defence. Genome sequencing revealed that Phytophthora species have a large number of genes predicted to encode RXLR effectors (Haas et al., 2009; Stassen and van der Ackervecken, 2011). These effectors are named after a conserved motif consisting of the amino acids arginine - any amino acid - leucine arginine (RXLR) and are secreted during infection (Bouwmeester et al., 2009; Stassen \& van der Ackerveken, 2011; Whisson et al., 2016). The RXLR motif is located in the N-terminal part adjacent to the signal peptide and precedes a highly variable $\mathrm{C}$-terminal region that determines effector activity and specificity (Bos et al., 2006; van Poppel et al., 2008; Jiang et al., 2008; Champouret et al., 2009).

Phytophthora species exploit multiple strategies to suppress plant defence. The many RXLR effectors target a vast array of host proteins thereby manipulating the host cell machinery at different levels and subcellular locations. For several $P$. infestans RXLR effectors, host targets have been identified. The RXLR effector IPI-O disrupts adhesion between the plant plasma membrane (PM) and the cell wall, potentially via interacting with lectin receptor kinases (Bouwmeester et al., 2011). AVR3a was shown to target and stabilize the E3 ubiquitin ligase CMPG1 to suppress plant immunity (Bos et al., 2010), whereas Pi03192 prevents re-localization of two plant NAC transcription factors to the nucleus thereby suppressing defence activation (McLellan et al., 2013). PexRD2 was shown to interact with the kinase domain of MAPKKKE leading to disruption of the MAPK defense signaling pathway (King et al., 2014). Besides suppressing plant defence, several RXLR effectors are recognized by intracellular nucleotide-binding leucine-rich repeat (NLR) resistance (R) proteins, and hence called avirulence (AVR) proteins. This effector-triggered immunity results in a hypersensitive response (HR) manifested by localized cell death to restrict pathogen invasion (Jones and Dangl, 2006). This is for example the case for AVR3a that is encoded by two alleles that only differ in three amino acids. Avirulent $P$. infestans isolates express the Avr3a allele which encodes for AVR3a ${ }^{\mathrm{Kl}}$ that is recognized by NLR R-protein R3a thereby triggering $\mathrm{HR}$, while virulent isolates express the avr3a allele encoding AVR3a $\mathrm{E}^{\mathrm{EM}}$ that escapes recognition by $\mathrm{R} 3 \mathrm{a}$ (Bos et al., 2006).

Similarly, RXLR effector AVR1 from $P$. infestans is recognized by the corresponding potato NLR R-protein R1 and this triggers a strong HR resulting in R1-mediated resistance (Du et al., 2015b). P. infestans isolates able to colonize $R 1$-potato plants were found to lack AVR1, but do contain a homolog named AVR1-like (A-L) (Du et al., 2015b; Du et al., 2018b). A-L is highly similar to AVR1 ( $82 \%$ homology at the protein level), but is shorter then AVR1 because it lacks the T-region, a tail of 38 amino acids at the very end of the C-terminus. A-L is not recognized by $\mathrm{R} 1$ and therefore isolates carrying $A-L$ instead of $A V R 1$ do not trigger R1-mediated HR (Du et al., 2018b). 
During infection of a non- $R 1$ potato cultivar, AVR1 acts as a virulence factor: it promotes colonization and suppresses defence responses in the host including callose deposition (Du et al., 2015b). It does so by targeting the potato protein StSec5 (Du et al., 2015a). Sec5 is a subunit of the exocyst, an octameric protein complex mediating polarized exocytosis by tethering exocytotic vesicles to the plasma membrane (Ravikumar et al., 2017). In plants, the exocyst functions in several developmental and defence-related processes, including cytokinesis, polarized tip growth and callose deposition (Zarsky et al., 2013; Martin-Urdiroz et al., 2016; Du et al., 2018a; Kulich et al., 2018; Guo et al., 2018). Co-immunoprecipitation assays have shown that in plant cells (in this case in Nicotiana benthamiana) AVR1 directly interacts with StSec5. These assays also revealed that in the presence of AVR1, the amount of detectable StSec5 was increased, suggesting that AVR1 stabilizes StSec5 (Du et al., 2015a). The finding that both Sec5 silencing and transient expression of AVR1 results in a decrease in callose deposition, raised the hypothesis that the AVR1-Sec5 interaction impairs exocyst function (Du et al., 2015a). In contrast to AVR1, A-L and AVR1 lacking the T-region (AVR1 ${ }^{\Delta \mathrm{T}}$ ) were not able to interact with $\mathrm{StSec5}$, highlighting the importance of the T-region in host defence suppression (Du et al., 2015a).

Besides AVR1, a few other pathogen effectors were found to target exocyst subunits. One interaction that is confirmed by co-immunoprecipitation is between AVR-Pii from the rice blast fungus Magnaporthe oryzae and rice Exo70, in particular Exo70F3 (Fujisaki et al., 2015). Other interactions that scored positive in yeast-two-hybrid (Y2H) screens include the RXLR effectors RxL62 and RxL470 from the oomycete Hyaloperonospora arabidopsidis and the type III effector HopC1 from the bacterium Pseudomonas syringae, that all three potentially interact with Arabidopsis thaliana Sec5a (Wessling et al., 2014), but these await further confirmation. The same holds for the interaction of $P$. infestans IPI-O with the exocyst subunit Sec10 identified by mass spectroscopy in an IPI-O pull down complex (unpublished data).

In this study we exploit the AVR1-Sec5 interaction to analyse possible mechanisms by which pathogen effectors manipulate exocyst functioning. We make use of a novel pathosystem that we developed previously with the moss Physcomitrella patens as host for Phytophthora pathogens (Overdijk et al., 2016). P. patens is ideal for high resolution microscopy and therefore we used it to investigate whether AVR1 affects Sec5 subcellular localization and exocyst functioning. First, we selected suitable P. patens Sec5 homologs by testing their interaction with AVR1. We then expressed AVR1 in P. patens lines with GFPtagged Sec5 using a $\beta$-estradiol inducible expression system and investigated the effect of AVR1 on P. patens growth and Sec5 localization. 


\section{Material and methods}

\section{Protein alignment and phylogenetic analysis}

Protein sequence alignments were constructed using MAFFT and edited in Jalview. Phylogenetic trees were constructed using Phylogeny.fr and the percent identity matrix with Clustal Omega. Gene codes are listed in Supplementary Table S1.

\section{Plasmids and cloning procedures}

For yeast-two-hybrid expression constructs, coding sequences of $\operatorname{Sec} 5 a, \operatorname{Sec} 5 b, \operatorname{Sec} 5 c$ and Sec5d were PCR-amplified from a P. patens cDNA library (Gene IDs in Table S1). Sec5c was manually re-annotated: the first 616 bp's from genome assembly V1.6 were put in front of the coding sequence of V3.1 (retrieved from cosmoss.org). PCR products were cloned into the pENTR-D-TOPO vector and subsequently subcloned into destination plasmid pDEST22 (Invitrogen) via Gateway LR reactions. pDEST32 containing AVR1, A-L and $A V R 1^{\Delta T}$ were described in a previous study (Du et al., 2015b). The 4-myc-Cerulean-AVR1 and 4-myc-Cerulean$A V R 1^{\Delta T}$ constructs were created by overlap PCR and subsequently introduced into the pENTRD-TOPO vector and recombined into destination plasmid PDEST32 via Gateway LR reactions.

For $\beta$-estradiol inducible AVR1 expression in P. patens, we used the system published by Kubo et al. (2013). To generate a myc-tagged AVR1, we amplified AVR1-10myc from a pGWB20AVR1-myc vector (Du et al., 2015b) and introduced this into the pENTR-D-TOPO vector. The same was done for AVR1 $\Delta T-10 m y c$ after recombination of the pENTR-D-TOPO-AVR1 ${ }^{\Delta T}$ with pGWB20 (Invitrogen) via LR cloning. The pENTR-D-TOPO vectors containing AVR1myc, $A V R 1^{\Delta \mathrm{T}}$-myc, myc-Cer-AVR1 or myc-Cer-AVR $1^{\Delta \mathrm{T}}$ were subsequently recombined into the $\beta$-estradiol inducible expression vector PPGX8 (Kubo et al., 2013) via Gateway LR reactions.

For $\beta$-estradiol inducible RNAi targeting of the four PpSec5 transcripts, we used the system published by Nakaoka et al. (2012). Four 450 base pair fragments of the coding sequence of Sec5a, Sec5b, Sec5c and Sec5d were amplified by PCR from a P. patens CDNA library and cloned into pENTR-D-TOPO and subsequently introduced into silencing vector pGG626 (Nakaoka et al., 2012) via a Gateway LR reaction (Supplementary Figure S3A). Used primers and plasmids are listed in Supplementary Table S2 and S3.

\section{Yeast-two-hybrid assays}

Yeast-two-hybrid assays were performed with a split Gal4 transcription factor system using the His3 gene as reporter (James et al., 1996). PpSec5 proteins were fused with the Gal4 activation domain (AD: pDEST22) and AVR1 proteins with the binding domain (BD: pDEST32). For this, pDEST22/32-based constructs (Supplementary Table S3) were transformed into yeast strain PJ69-4a or PJ69-4a by PEG-mediated transformation. Positive yeast transformants with minimal background reporter activity were selected on double dropout medium (-Leu-His or -Trp-His) with different concentrations of 3-amino-1,2,4triazole (3-AT) to increase histidine-dependent growth stringency. Selected clones were 


\section{Chapter 5}

mated and resulting diploids were selected on -Leu-Trp plates. With the surviving cells, a yeast-two-hybrid assay was performed on triple dropout medium (-Leu-Trp-His) with increasing concentrations of 3-AT.

\section{P. patens growth conditions and transformation}

P. patens was grown on BCDAT plates under continuous light at $25^{\circ} \mathrm{C}$ (Nishiyama et al. 2000). Plasmids were linearized and introduced into the P. patens genome by homologous recombination using PEG-mediated protoplast transformation (Nishiyama et al. 2000). Correct insertion events were characterised by PCR (Supplementary Figure S1). Generated moss lines are listed in Supplementary Table S4.

\section{P. patens phenotyping}

For phenotyping at the colony level, $P$. patens protonema of about $1 \mathrm{~mm}^{2}$ in size was grown on BCDAT medium covered with cellophane for two days. Subsequently, half of the replicas was transferred to BCDAT medium enriched with $1 \mu \mathrm{M} \beta$-estradiol, the other half was used as non-induced control. Growth of the colony was visualised at 0, 3 and 5 days after $\beta$-estradiol induction. P. patens cell morphology was visualised 3 days after culturing in special glass bottom dishes (Yamada et al., 2016) containing BCD medium with and without $\beta$-estradiol.

\section{Quantitative RT-PCR}

P. patens protonemal tissue was grown on BCDAT medium with and without $\beta$-estradiol for 2 days and total RNA was isolated using Trizol and a RNeasy mini kit (Qiagen). CDNA was synthesized on $1 \mu \mathrm{g}$ of total RNA using a iScript cDNA synthesis kit (Bio-Rad). Quantitative RT-PCR was performed using a SYBR Hi-ROX kit (Bioline, London, UK), gene-specific primers (Supplementary Table S2) and $3 \mu$ l of 10-times diluted cDNA using a Bio-Rad CFX96 RealTime PCR system. Gene expression levels were normalized to EF1a transcript levels and to the transcript levels of the studied gene in non-induced moss tissue.

\section{Microscopy}

Phenotyping was performed using a Nikon Hoffman modulation contrast microscope equipped with an Axiocam MRc5 with Axiovision software and 4 x (Leitz Wetzlar, NA 0.12) or $20 \times$ (Nikon MC2, NA 0.4) objectives.

Fluorescent live-cell imaging was performed on a Roper spinning disk microscope system composed of a Nikon Ti eclipse body, Yokogawa CSU-X1 spinning disc head and a Photometrics Evolve 512 camera. Imaging was conducted with a 100x Plan Apo VC oil immersion objective (NA 1.40) using a 1.2x post-magnification fitted in front of the camera. GFP was exited using $491 \mathrm{~nm}$ light generated by a Cobolt Calypso50 laser, 30\% power, and the emitted light was bandpass filtered at 497-560 nm. Cerulean was excited using a $405 \mathrm{~nm}$ Melles-Griot 56RCS001 laser, 85\% power, and emission light was filtered through 
a 460/50 band pass filter. Typical exposures were 1000-2000 ms. For live-cell imaging of infected cells, P. patens was infected with P. capsici isolate LT263 and subsequently imaged by time-gated microscopy as described in Overdijk et al., 2016 and Chapter 4. FIJ software (Schindelin et al., 2012) was used for all image analysis and processing.

A

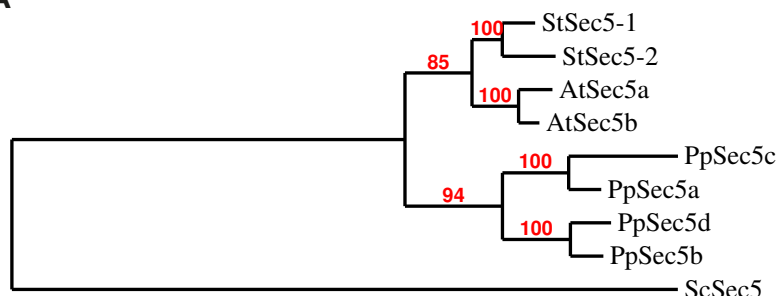

0.8

B

\begin{tabular}{|c|c|c|c|c|c|c|c|c|}
\hline & AtSec5a & AtSec5b & StSec5-1 & StSec5-2 & PpSec5a & PpSec5b & PpSec5c & PpSec5d \\
\hline AtSec5a & 100 & & & & & & & \\
\hline AtSec5b & 81 & 100 & & & & & & \\
\hline StSec5-1 & 65 & 65 & 100 & & & & & \\
\hline StSec5-2 & 63 & 63 & 74 & 100 & & & & \\
\hline PpSec5a & 43 & 43 & 43 & 41 & 100 & & & \\
\hline PpSec5b & 43 & 44 & 43 & 41 & 53 & 100 & & \\
\hline PpSec5c & 37 & 38 & 38 & 37 & 65 & 47 & 100 & \\
\hline PpSec5d & 43 & 43 & 43 & 42 & 54 & 76 & 46 & 100 \\
\hline
\end{tabular}

C

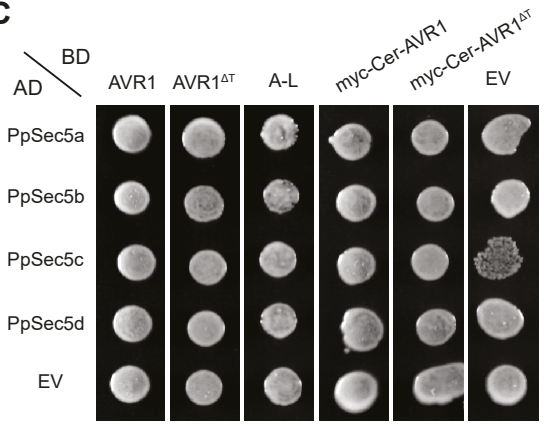

- Leu - Trp

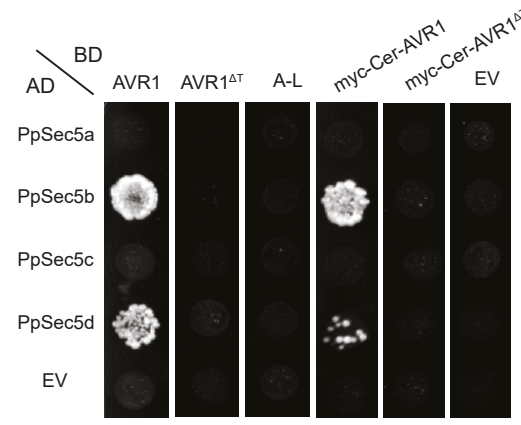

- Leu - Trp - His + 10 mM 3-AT

FIGURE 1. P. infestans effector AVR1 interacts with P. patens homologs Sec5b and Sec5d. (A) Phylogenetic tree of Sec5 proteins of P. patens (Pp), A. thaliana (At) and potato (Solanum tuberosum, St). Yeast (Saccharomyces cerevisiae, Sc) Sec5 served as an outgroup. The phylogram was generated using the maximum likelihood method. Bootstrap values (red) indicate the percentage of trees in which the associated taxa cluster. Horizontal branch length represents the number of amino acid substitutions. Gene codes are listed in Table S1. (B) Percent identity matrix of the Sec5 proteins. (C) Yeast-two-hybrid assay to analyse the interaction between the four $P$. patens Sec 5 homologs and different versions of AVR1. Proteins were fused with the Gal 4 activation domain (AD) and binding domain (BD), respectively. Yeast strains co-expressing the different combinations were tested for successful mating on double dropout medium (-Leu-Trp) and for growth on triple dropout medium (-Leu-TrpHis) supplemented with $10 \mathrm{~m} \mathrm{M}$ of 3-AT. EV: empty vector. 


\section{Results and discussion}

\section{AVR1 interacts with the P. patens Sec5 homologs PpSec5b and PpSec5d}

The $P$. patens genome contains four Sec5 genes encoding proteins that share about $40 \%$ identity with Sec5 subunits of seed plants (Figure 1). To determine whether AVR1 interacts with $P$. patens Sec5 we performed yeast-two-hybrid $(\mathrm{Y} 2 \mathrm{H})$ assays and tested all four $P$. patens Sec 5 homologs for their interaction with AVR1, AVR1 ${ }^{\Delta \top}$ and A-L. The assays showed that AVR1 interacts with two Sec5 homologs, PpSec5b and PpSec5d, while AVR1 ${ }^{\Delta T}$ and A-L do not interact with any of the four PpSec5s (Figure 1C). The latter is in line with results of Du et al. (2015a) showing that truncation of the T-region abolishes the interaction with StSec5 (Du et al., 2015a). The binding to only two out of four Sec5 homologs might be due to the sequence divergence among the homologs. PpSec5b and PpSec5 $d$ group together in the phylogenetic tree separated from the other two, and have the highest pairwise similarity (76\%) (Fig. 1A and 1B). Alignment of the four PpSec5 protein sequences with StSec5 revealed over 40 amino acids that are identical in StSec5, PpSec5b and PpSec5d but different in PpSec5a and PpSec5c (Supplementary Figure S2). Whether or not these amino acids are determinants for the structure of Sec5 and/or the molecular interaction with AVR1 remains to be investigated.

\section{$A V R 1$ and $A V R 1^{\Delta T}$ expression in P. patens}

To study whether or not the presence of AVR1 inside plant cells affects the behaviour of Sec5, we generated transgenic P. patens lines expressing AVR1 or, as a control, $A V R 1^{\Delta T}$. To avoid the risk of AVR1 causing developmental defects, thereby disturbing the transformation process, we used an inducible expression system mediated by $\beta$-estradiol (Kubo et al., 2013). We also included tags to facilitate detection of the proteins, i.e. a 10-myc-tag at the C-terminus or a 4-myc-tag combined with a fluorescent Cerulean-tag (Cer) at the N-terminus (Supplementary Figure S1). As shown previously the AVR1-myc fusion protein is still able to interact with $\mathrm{StSec} 5$ in $N$. benthamiana despite the presence of the tag (Du et al., 2015a). Similarly, the myc-Cer-AVR1 fusion protein still interacts with $\mathrm{PpSec} 5 \mathrm{~b}$ and PpSec5d in the $\mathrm{Y} 2 \mathrm{H}$ assay (Figure $1 \mathrm{C}$ ), indicating that the $\mathrm{N}$-terminal tag does not abolish the interaction of AVR1 with its target.

The $A V R 1$ and $A V R 1^{\Delta T}$ constructs were integrated via homologous recombination in two previously developed $P$. patens recipient lines; one containing Sec5b-GFP and the other Sec5d-GFP (Tang et al., 2019). We selected about hundred stable transformants and verified the insertions of the four constructs by genotyping (Supplementary Figure S1). Successful induction of $A V R 1$ and $A V R 1^{\Delta T}$ expression upon $\beta$-estradiol treatment was confirmed by confocal fluorescence microscopy (Figure 2). Myc-Cer-AVR1 and myc-CerAVR1 ${ }^{\Delta T}$ localized to the cytoplasm and nucleus of induced $P$. patens cells. Non-induced cells consistently showed chloroplast autofluorescence and no other fluorescence demonstrating that expression of $A V R 1$ and $A V R 1^{\Delta T}$ is tightly regulated via $\beta$-estradiol 
induction. The nucleocytoplasmic localization of AVR1 in P. patens is in line with previous findings in N. benthamiana (Du et al., 2015b; Wang et al., 2018) and characteristic for AVR1 function. Activation of R1-mediated immunity requires AVR1 localization in the nucleus, while in the absence of R1, host defence suppression is more effective when AVR1 is targeted to the cytoplasm (Du et al., 2015b).

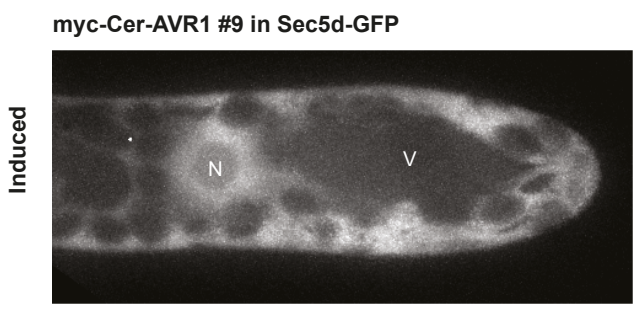

myc-Cer-AVR1 ${ }^{\Delta \mathrm{T}} \# 3$ in Sec5d-GFP
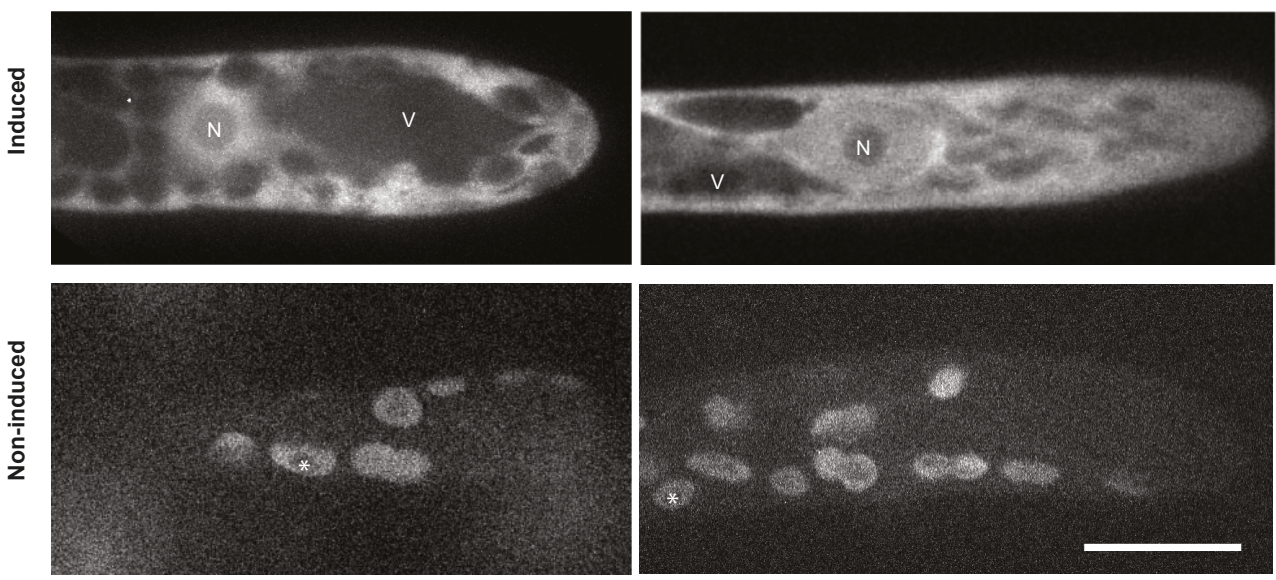

FIGURE 2. AVR1 and AVR1 ${ }^{\Delta T}$ localize in the cytoplasm and nucleus of $\boldsymbol{P}$. patens. Moss lines were grown for 3 days on BCD medium with or without $\beta$-estradiol (induced and non-induced, respectively) and observed by confocal fluorescence microscopy. Images are maximum z-projections of 5 slides, $0.5 \mu \mathrm{m}$ each, for images in the upper panel and single confocal planes for images in the lower panel. Scale bar represents $10 \mu \mathrm{m}$. N: nucleus, V: vacuole. Asterisks mark auto-fluorescence of chloroplasts in the lower panel.

\section{AVR1 and AVR $1^{\Delta T}$ affect growth and development of $P$. patens protonema cells}

To assess the effects of AVR1 on $P$. patens, we monitored a large number of independent AVR1 and AVR1 $1^{\Delta T} P$. patens lines on medium with and without $\beta$-estradiol (Figure 3 ). Compared to non-induced lines, AVR1-expressing P. patens lines showed reduced colony sizes and the conspicuous hairy appearance was far less prominent (mild phenotype) or even absent (severe phenotype) (Figure 3B and $3 C$ ). Remarkably, moss lines expressing $A V R 1^{\Delta T}$ showed the same phenotype. This is caused by a reduction of caulonema cells, which are fast-growing protonema cells with poorly developed chloroplasts (Menand et al., 2007). Besides the reduced colony size, we also observed defects in cell morphology (Figure 3D). Chloronema cells of AVR1 or AVR $1^{\Delta T}$ expressing lines consistently showed abnormal swelling (Figure $3 \mathrm{D}$ and $3 \mathrm{E}$ ). It should be noted that the control recipient moss line, without an AVR1 or AVR $1^{\Delta T}$ transgene, also showed some reduction in colony size upon transfer to plates containing $\beta$-estradiol, but this is just a small fraction of the severe growth reduction observed in the transgenic lines. We suspect that this minor growth reduction is due to some minor damage caused by the transfer itself and not by $\beta$-estradiol. Taken together, these results show that the presence of AVR1 or AVR1 ${ }^{\Delta \mathrm{T}}$ inside the cell disturbs P. patens cell growth and development. 
The finding that the presence of either AVR1 or AVR1 ${ }^{\Delta T}$ affects moss development in a similar way could be interpreted in two ways. If we assume that AVR1 ${ }^{\Delta T}$ does not bind PpSec5s in planta, as predicted from the $\mathrm{Y} 2 \mathrm{H}$ assay, then it is unlikely that the growth phenotype is caused by direct interaction with Sec5. In that case both AVR1 and AVR1 ${ }^{\Delta T}$ potentially interfere with other processes involved in tip growth. It not uncommon that a pathogen effector targets multiple host proteins and this could be true for AVR1 as well (Wessling et al., 2014; Khan et al., 2018). In fact, in coimmunoprecipitation experiments we found that AVR1 interacts with several proteins in N. benthamiana, including multiple clathrin heavy chain homologs (unpublished data). Clathrin-mediated endocytotic membrane trafficking is essential for plant development and tip cell growth by balancing exocytosis (Grebnev et al., 2017) and this could explain the cell growth defect in P. patens caused by AVR1. However, unlike AVR1, AVR1 ${ }^{\Delta T}$ does not suppress cell death induced by CRINKLERs, another class of $P$. infestans effectors, and it does not promote $P$. infestans colonization (Du et al., 2015a), making it less likely that AVR1 ${ }^{\Delta \mathrm{T}}$ and AVR1 interact with the same host target. The second option is that AVR $1^{\Delta T}$ does bind to PpSec5s, but with a lower affinity than AVR1. Although negative in the $\mathrm{Y} 2 \mathrm{H}$ assay, we cannot exclude that there is interaction of AVR1 ${ }^{\Delta T}$ with PpSec5s in planta. As yet, this interaction in planta has not been tested also not between AVR1 ${ }^{\Delta \mathrm{T}}$ and StSec5. If there is binding to PsSec5, the highly similar phenotypes in the independent $P$. patens lines expressing AVR1 or AVR $1^{\Delta \mathrm{T}}$ indicate that this binding is responsible for the defects in cell growth and development.

\section{Sec5- or Sec6-silenced P. patens lines are phenocopies of AVR 1-expressing lines}

Since AVR1 disrupts Sec5 functioning in potato (Du et al., 2015a), we anticipated that downregulation of Sec5 would cause the same effect as expression of AVR1. We therefore made a $\beta$-estradiol inducible RNAi construct that targets transcripts of all four Sec5 homologs of P. patens for degradation (Supplementary Figure S3) and introduced this into the neutral P. patens PIG1 locus via homologous recombination (Nakaoka et al., 2012). The knock-down levels in stable transformed moss lines were validated by quantitative RT-PCR and this showed a reduction of about $40 \%$ in gene expression for all four PpSec 5 homologs (Supplementary Figure S3). Sec5-silencing resulted in reduced caulonemal growth (Figure 4A) and chloronemal tissue with swollen cells (Figure 4B, arrow heads). This swelling may be a consequence of disrupted polarized growth. Disruption of polarized cell growth is a defect that has been associated with exocyst functioning in e.g. pollen tubes and root hairs in seed plants (Bloch et al., 2016; Sekeres et al., 2017), thus pointing to impairment of exocyst functioning in Sec5-silenced lines. The phenotypic similarities between AVR1expressing and Sec5-silenced $P$. patens lines (compare Figure 3 and 4), suggests that the presence of AVR1 disrupts Sec5 functioning. 
A

Background AVR1-myc AVR1 ${ }^{\Delta T}$-myc myc-Cer-AVR1 myc-Cer-AVR1 ${ }^{\Delta T}$

\begin{tabular}{ccccc}
\hline Sec5b-GFP & 14 & 15 & 13 & 8 \\
\hline Sec5d-GFP & 8 & 3 & 13 & 23 \\
\hline Total transformants & 22 & 18 & 26 & 31
\end{tabular}

\section{B}

AVR1-myc \#66 in Sec5b-GFP

AVR1 ${ }^{\Delta T}$-myc \#224

myc-Cer-AVR1 \#119 in Sec5b-GFP

myc-Cer-AVR1 ${ }^{\Delta \top} \# 17$

in Sec5b-GFP

Sec5b-GFP

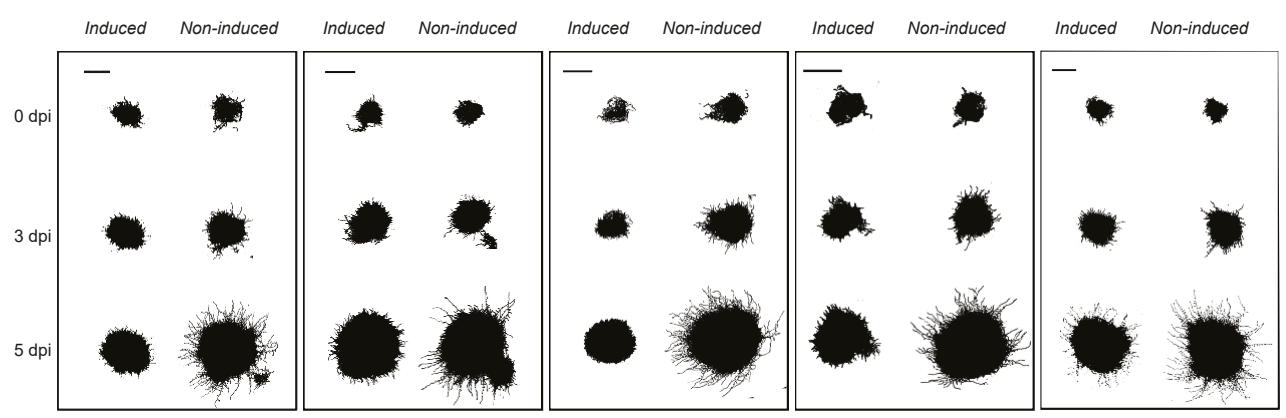

C

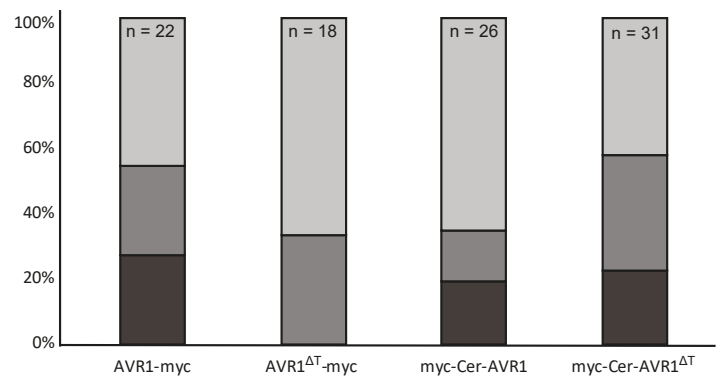

D
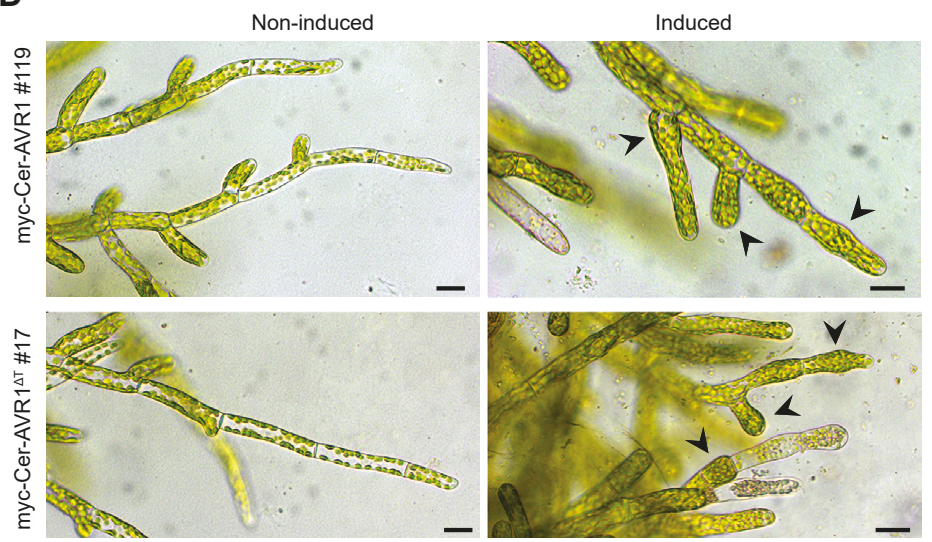

E

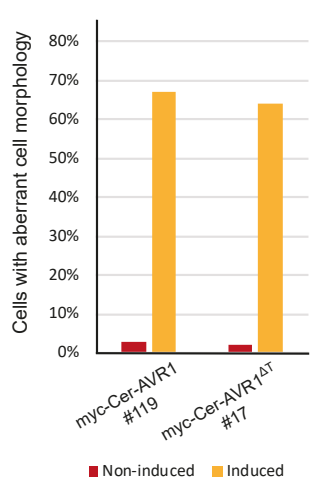

FIGURE 3. AVR1 and AVR1 ${ }^{\Delta T}$ reduce $P$. patens growth (legend continues on next page). (A) Total number of stable transgenic $P$. patens lines containing inducible AVR1 or AVR $1^{\Delta T}$. Four different constructs (AVR1-myc, $A V R 1^{\Delta T}$-myc, myc-Cer-AVR1 and myc-Cer-AVR $1^{\Delta T}$ ) were transformed into two background P. patens lines (Sec5bGFP and Sec5d-GFP). (B) P. patens growth shown for representative transformants. Moss lines were grown on $B C D A T$ medium with and without $\beta$-estradiol and colony growth was visualised at 0,3 and 5 days after induction 


\section{Chapter 5}

(dpi). This experiment was repeated three times and representative images are shown. Scale bars represents 2 $\mathrm{mm}$. (C) Phenotype index of all AVR1 transformants. Transformants were grown on BCDAT medium with and without $\beta$-estradiol for 7 days and moss colony phenotypes were scored by microscopy. The phenotype was scored as severe when there was no caulonema outgrowth from the moss colony (e.g. myc-Cer-AVR1 \#119 in B) and as mild when the caulonema outgrowth was at least $30 \%$ reduced compared to the non-induced colony (e.g. AVR1 ${ }^{\Delta T}$-myc \#224 in B). (D) Cell morphology visualised by microscopy 3 days after induction with $\beta$-estradiol on BCD medium. Arrow heads point to aberrant cell morphology. Scale bars represents $10 \mu m$. (E) Frequency of aberrant cell morphologies observed in the experiment described in $D$ ( $n>70$ cells).

To test whether the observed defects in Sec5-silenced lines are caused by the function of Sec5 as part of the exocyst complex or by exocyst-independent roles of Sec5, we compared the phenotypes of Sec5-silenced lines with that of a Sec6-silenced line that was previously generated by Tang et al. (2019). Sec6 is also part of the exocyst complex and involved in the cytokinesis of $P$. patens (Tang et al., 2019). Consistent with the phenotype of Sec5-silenced lines, we observed that silencing of Sec6 results in reduced colony growth, absence of caulonemal cells and swelling of chloronema cells (Figure 4; Tang et al., 2019). Rawat et al. (2017) and van Gisbergen et al. (2018) studying P. patens Exo70.3d-mutants or Sec10-silenced P. patens lines, respectively, observed similar aberrant phenotypes in polarized tip growth and caulonema development. Taken together this shows that defects in caulonemal development and polarized cell growth are consistent in P. patens lines in which individual exocyst subunits are lacking or strongly reduced, pointing to malfunctioning of the exocyst. Therefore, we conclude that Sec5-silencing leads to (partial) disruption of the exocyst. Since the AVR1-expressing lines are phenocopies of the Sec5silenced lines, it is conceivable that AVR1 disrupts the exocyst by binding Sec5.

\section{AVR1 does not alter Sec5 localization}

To address the question what happens with Sec5 upon AVR1 binding, we investigated the subcellular localization of PpSec5b and PpSec5d in presence and absence of AVR1. We hypothesized that binding of AVR1 to Sec5 would alter the localization of Sec5 thereby disturbing its role in the exocyst complex. As a first step, we made an attempt to check whether AVR1 co-localizes with Sec5 in P. patens caulonema cells. Unfortunately, AVR1 was expressed at high-levels in the cell (Figure 2) making it impossible to determine whether AVR1 localizes to typical exocyst foci on the PM (Fendrych et al., 2013; Zhang et al., 2013; Bloch et al., 2016; Synek et al., 2017; Vukašinović et al., 2017; van Gisbergen et al., 2018; Chapter 4). We then investigated whether AVR1 alters the localization of Sec5b-GFP and Sec5d-GFP. After induction of AVR1 expression, we observed some variation in intensity of fluorescent foci at the PM, but the localization did not change; both Sec5b-GFP and Sec5d-GFP were visible as characteristic foci at the PM and this pattern was similar in absence of AVR1 (Figure 5). 
A

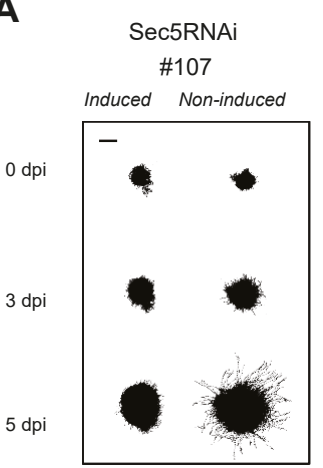

\section{Sec6RNAi}

\#6

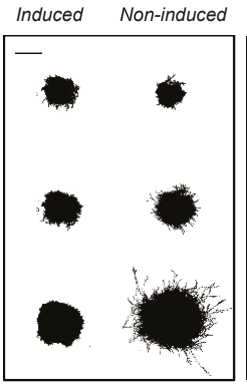

WT

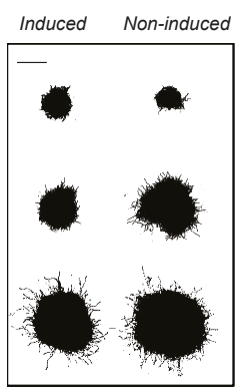

C

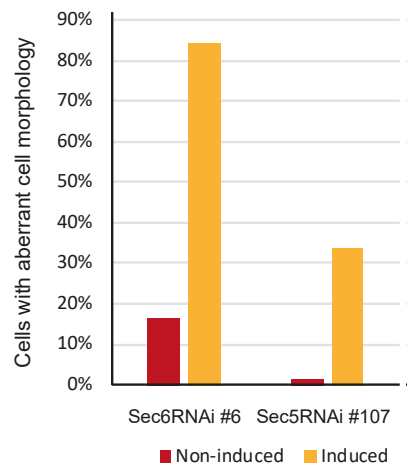

B Non-induced Induced
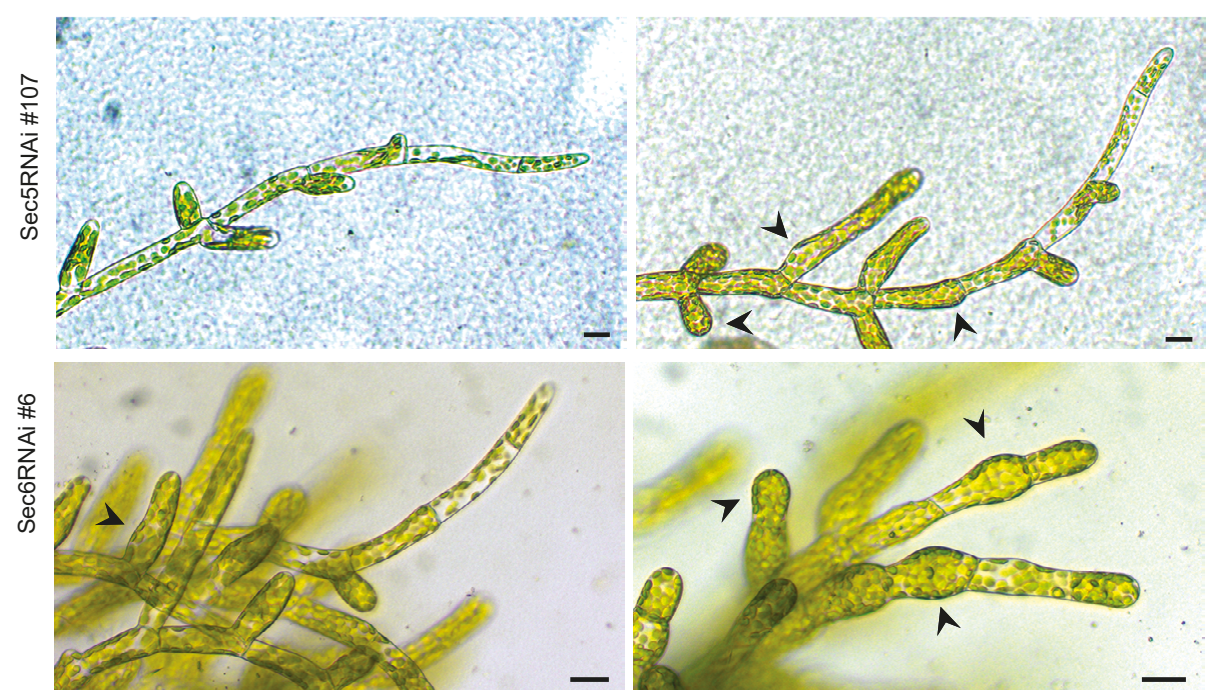

FIGURE 4. Sec5- or Sec6-silencing impairs P. patens growth and development. (A) P. patens Sec5RNAi and Sec6RNAi lines were grown on BCDAT medium with and without $\beta$-estradiol (induced and non-induced) and colony growth was visualised at 0,3 and $5 \mathrm{dpi}$. Scale bars represents $2 \mathrm{~mm}$. (B) Cell morphology was visualised by microscopy 3 days after induction with $\beta$-estradiol on BCD medium. Arrow heads point to aberrant cell morphology. Scale bars represents $10 \mu \mathrm{m}$. (C) Frequency of aberrant cell morphologies observed in the experiment described in $B(n>70$ cells). 

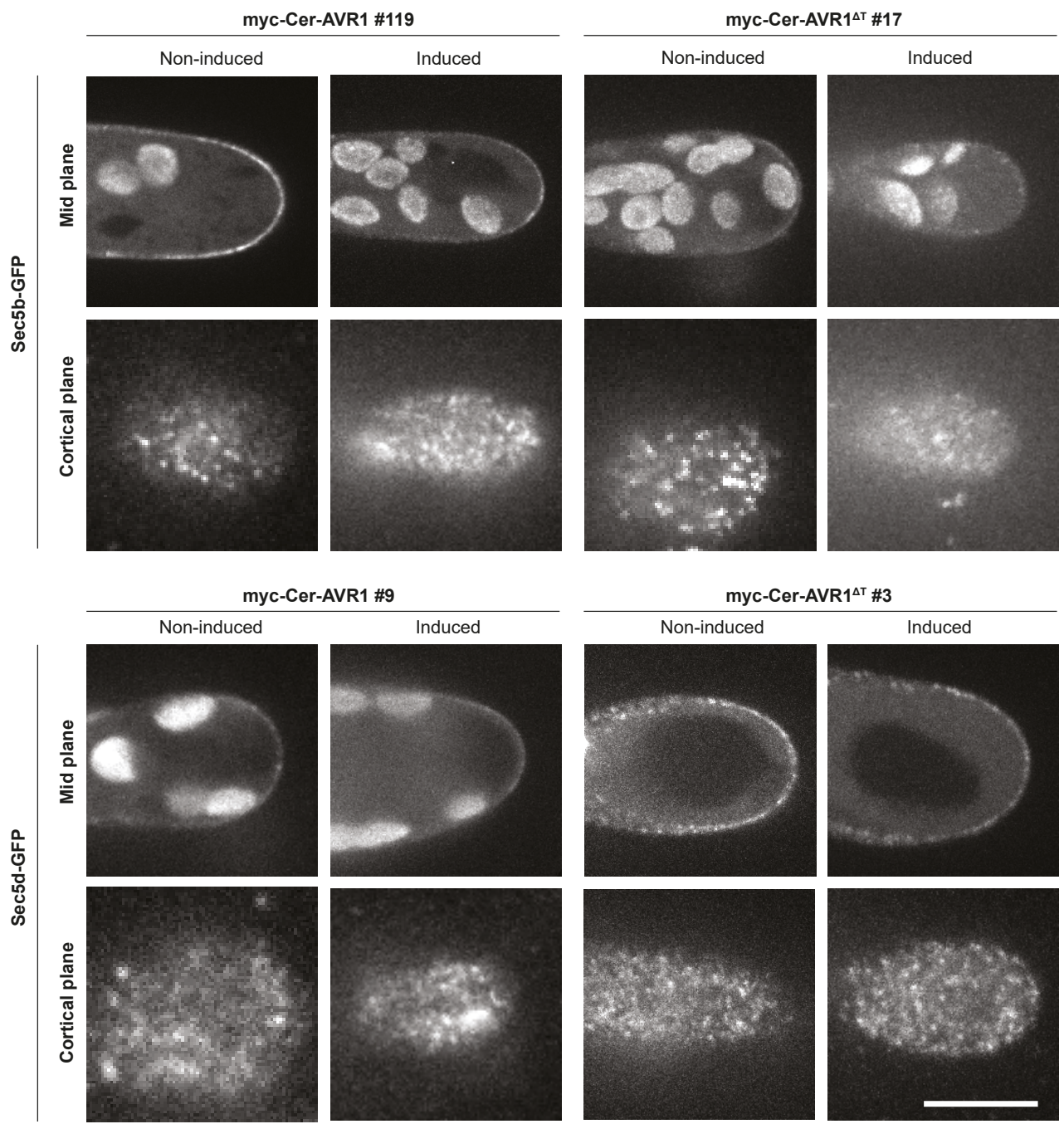

FIGURE 5. AVR1 and AVR1 ${ }^{\Delta T}$ do not alter Sec5 localization in non-infected cells. Subcellular localization of Sec5b-GFP and Sec5d-GFP visualised by confocal fluorescence microscopy. Mid planes are maximum $z$-projections of $2.5 \mu \mathrm{m}$ and cortical planes are average z-projections of $1.5 \mu \mathrm{m}$. Auto-fluorescence of chloroplasts is visible inside cells (mid plane). Scale bar rvepresents $10 \mu \mathrm{m}$.

When attacking potato, $P$. infestans secretes a large set of RXLR effectors that are deposited inside the plant cell. AVR1 is one of these effectors that normally ends up inside the plant cell during pathogen attack. We recently showed that in $P$. patens several exocyst subunits including Sec5b and Sec5d accumulate at Phytophthora infection sites (Chapter 4), and because of its binding to Sec5, AVR1 might play a role in redirecting this exocyst subunit. However, the Phytophthora species that was used in that study is $P$. capsici, a species that does not produce AVR1. To test whether AVR1 can disrupt or enhance recruitment of Sec5 to infection sites, we studied the localization of Sec5b in a P. patens line expressing AVR1 
inoculated with P. capsici. As shown in Figure 6, it appears that AVR1 does not affect the accumulation of Sec5b-GFP around infection sites. The localisation pattern is similar with or without AVR1 and autofluorescent material is still deposited in papilla-like structures (Figure 6 and Chapter 4). This suggests that AVR1 is not able to significantly suppress exocytosis during defence in $P$. patens despite the fact that AVR1 was shown to be a strong virulence factor (Du et al., 2015a).

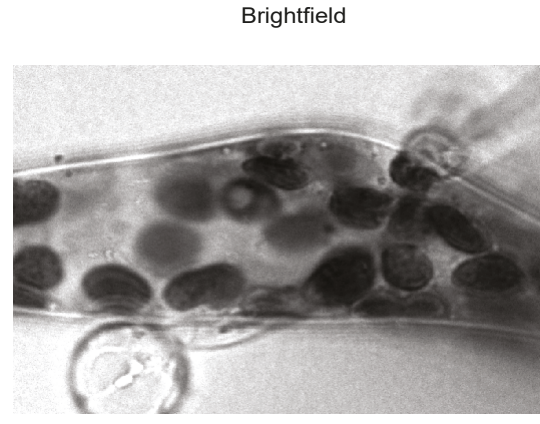

GFP-channel Gate open 1-12 ns

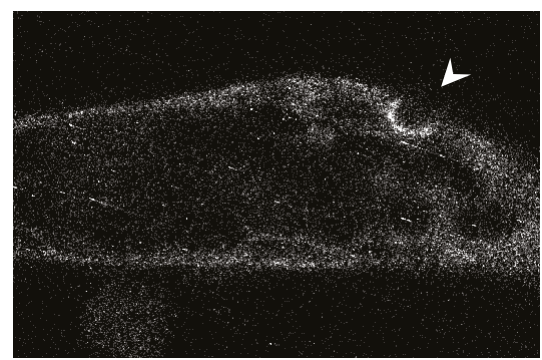

Autofluorescence No gating

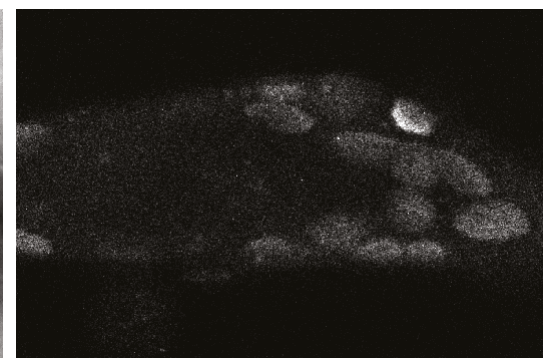

Overlay

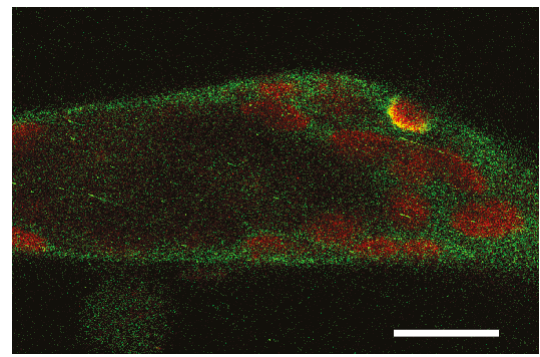

FIGURE 6. AVR1 does not disrupt Sec5b accumulation or the deposition of autofluorescent material at infection sites. Sec5b-GFP accumulation on the membrane surrounding a papilla-like structure (arrow head) in a P. capsici infected moss line (myc-Cer-AVR1 \#119). AVR1 expression was induced by growth on BCD medium with $\beta$-estradiol for 3 days prior to infection. Image shown is a single confocal plane taken 3 hours after pathogen inoculation. Scale bar represents $10 \mu \mathrm{m}$. 


\section{Chapter 5}

\section{Conclusion}

The cell growth defects of $P$. patens lines expressing AVR1 or AVR $1^{\Delta T}$ combined with the fact that this phenotype is copied in Sec5- or Sec6-silenced lines, indicate that AVR1 disrupts exocyst functioning in $P$. patens by binding to Sec5. However, AVR1 or AVR1 ${ }^{\Delta \mathrm{T}}$ did not alter the localization of Sec5, suggesting that the effect of the AVR1-Sec5 interaction on exocyst functioning is subtle, e.g. by slowing down exocyst dynamics or reducing exocyst efficiency. Attempts to quantify Sec5 dynamics by confocal fluorescence microscopy were unsuccessful, and it seems we need more advanced tools to determine how AVR1 modulates Sec5 functioning. Phytophthora species secrete a large number of effectors and it is known that pathogens need multiple effectors in order to effectively reduce plant defence (Haas et al., 2009; Khan et al., 2018). Therefore it is logical to assume that, besides AVR1, more effectors will be identified that target exocyst subunits and/or affect their subcellular localization. In potato and N. benthamiana, AVR1 acts as a virulence factor by promoting $P$. infestans colonization and supressing host defence responses such as the deposition of callose (Du et al., 2015a). In P. patens, quantification of the amount of deposited autofluorescent material at penetration attempt sites in the presence and absence of AVR1, could indicate whether AVR1 is also able to suppress exocytosis to these sites.

\section{Acknowledgements}

We would like to thank Richard Imminkand Marco Busscher (WUR-Bioscience) for assistance with $\mathrm{Y} 2 \mathrm{H}$ experiments, Yu Du for sharing plasmids and Henk Kieft and Raimo Paakkari for technical assistance. This research was supported by the Earth and Life Sciences Division of The Netherlands Organization for Scientific Research (NWO; PhD grant to EO). 

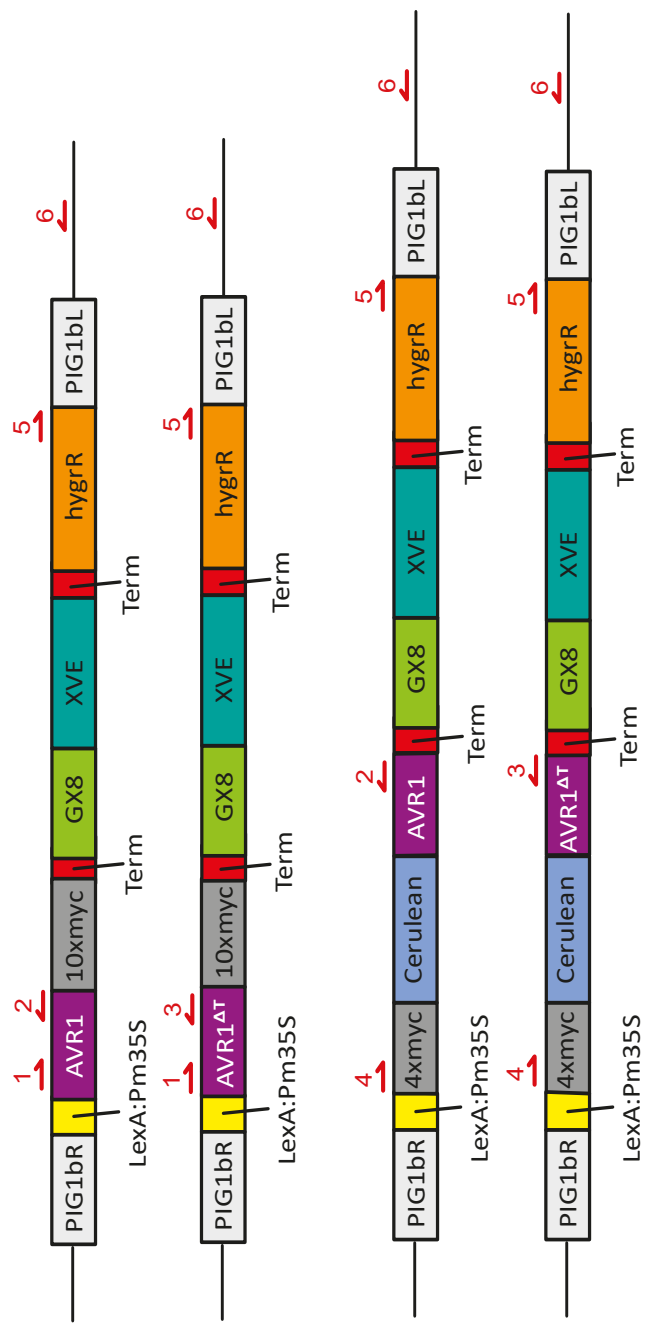

웅
乞ัنّ 空

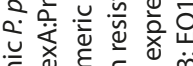

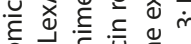

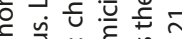
ฮั

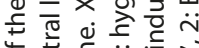

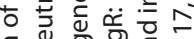
등 के

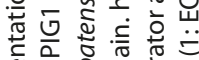

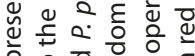

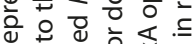

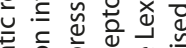

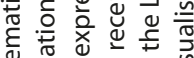
政

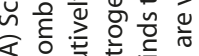

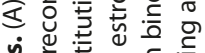
过芯芯志高

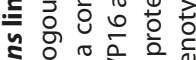

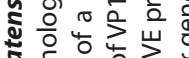

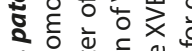

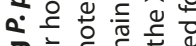

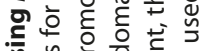

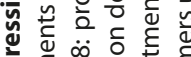

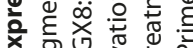

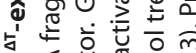

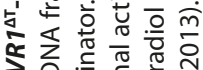

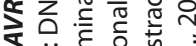

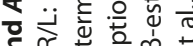
ता

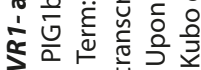

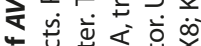
일

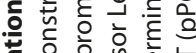

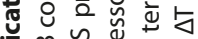

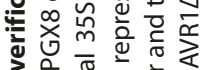
응

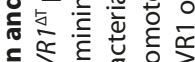

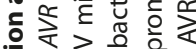

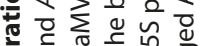
एँ

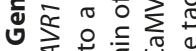

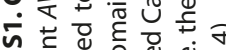

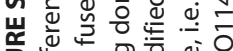

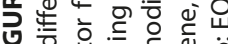
눈 후의

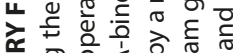

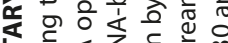

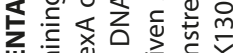

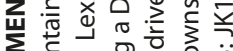

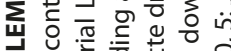
츤 촌 

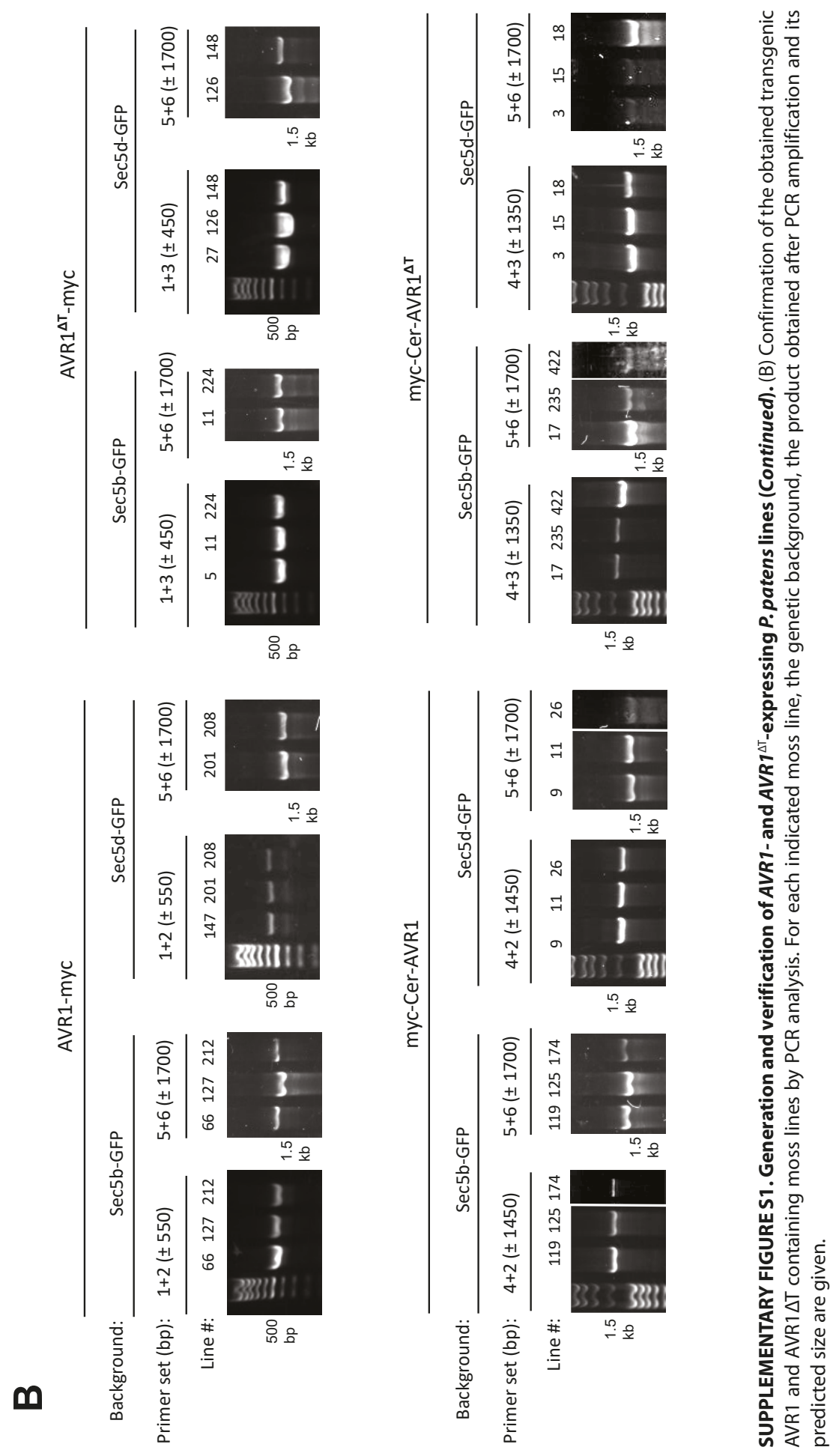


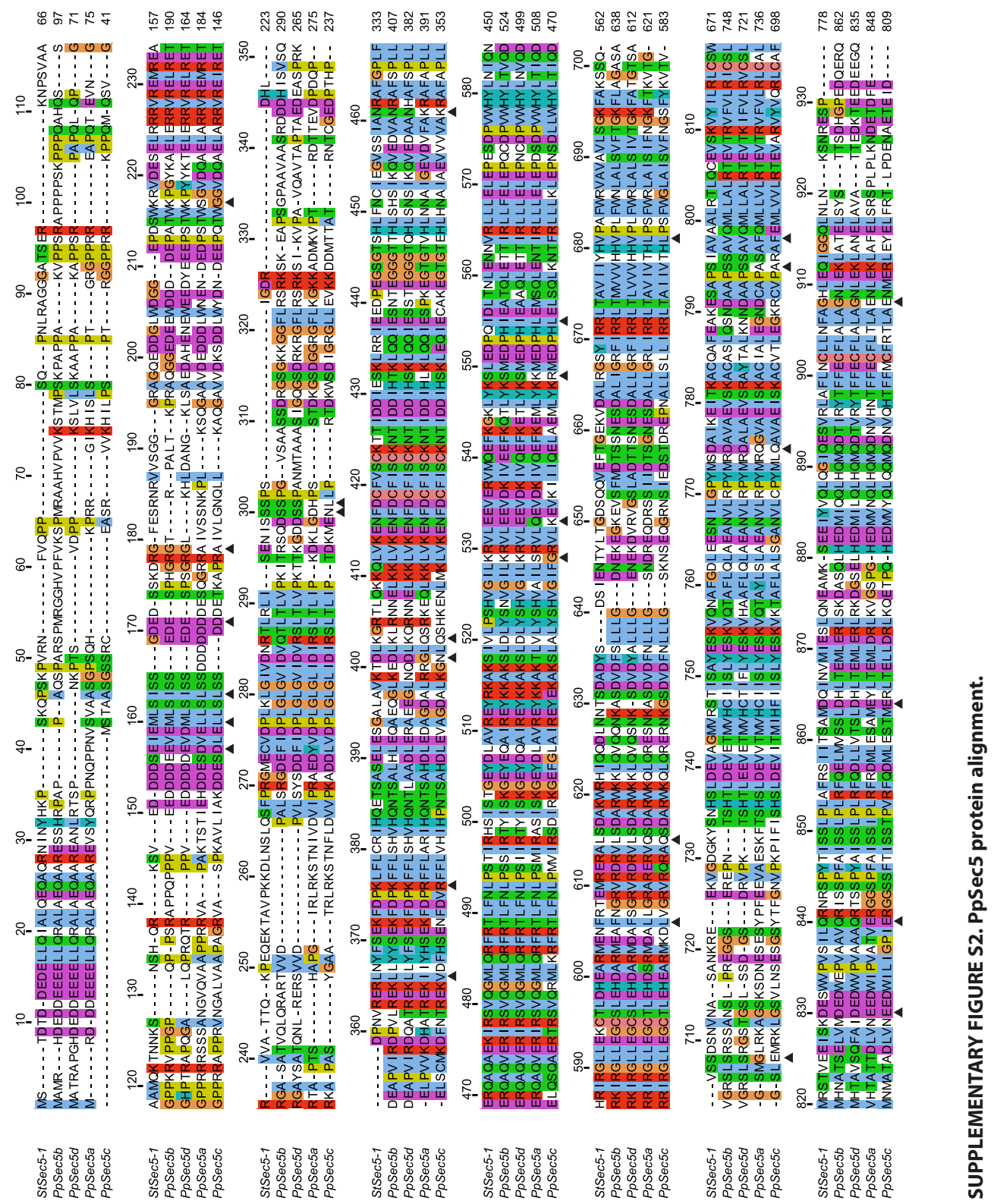




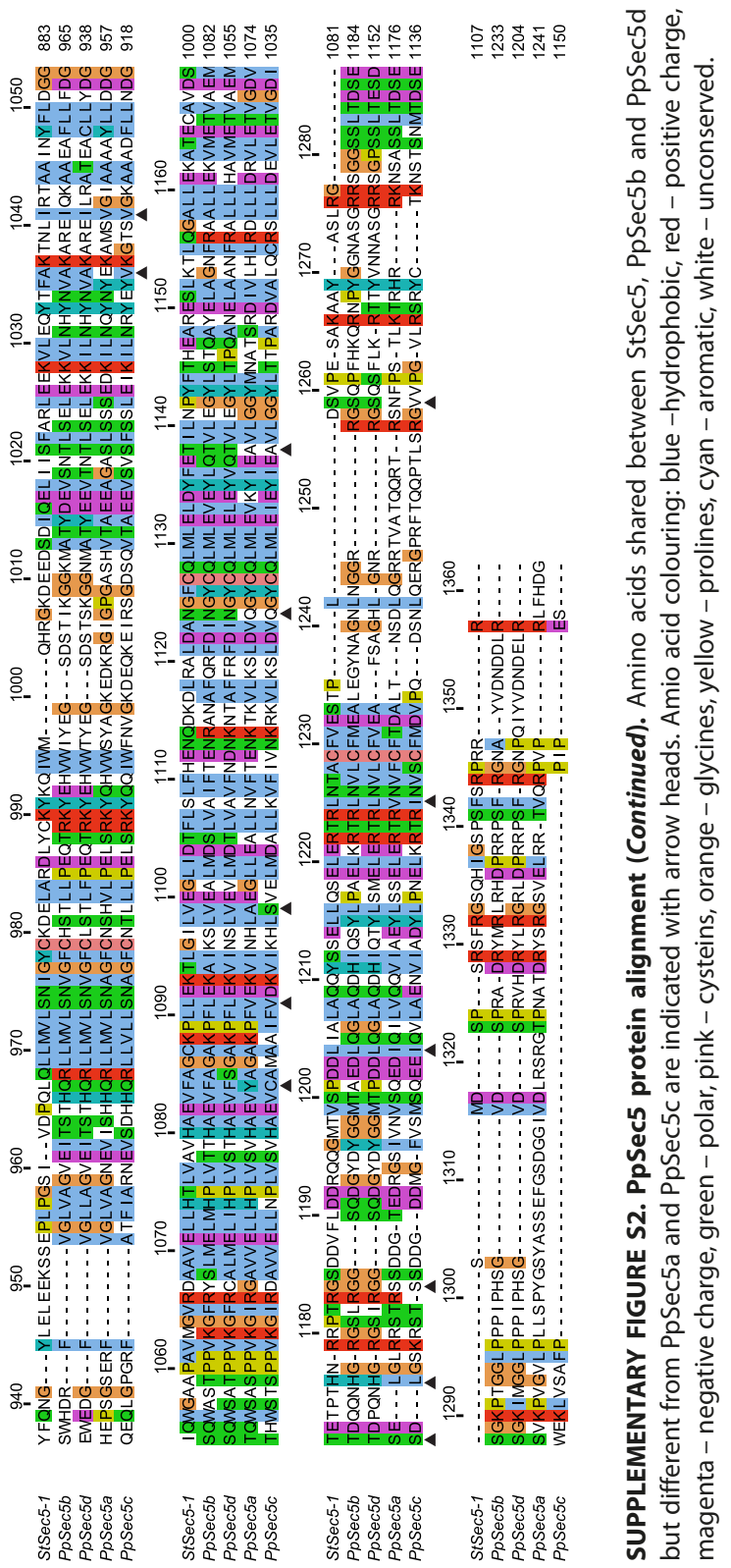




\section{A}

\begin{tabular}{|c|c|c|c|c|c|c|c|c|c|}
\hline Sec5RNAi & PIG1bR & $\sec 5 a$ & $\sec 5 c \sec 5 d$ & linker & 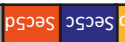 & es̃as & $\overline{X V E}$ & hygrR & PIG1bL \\
\hline
\end{tabular}

B

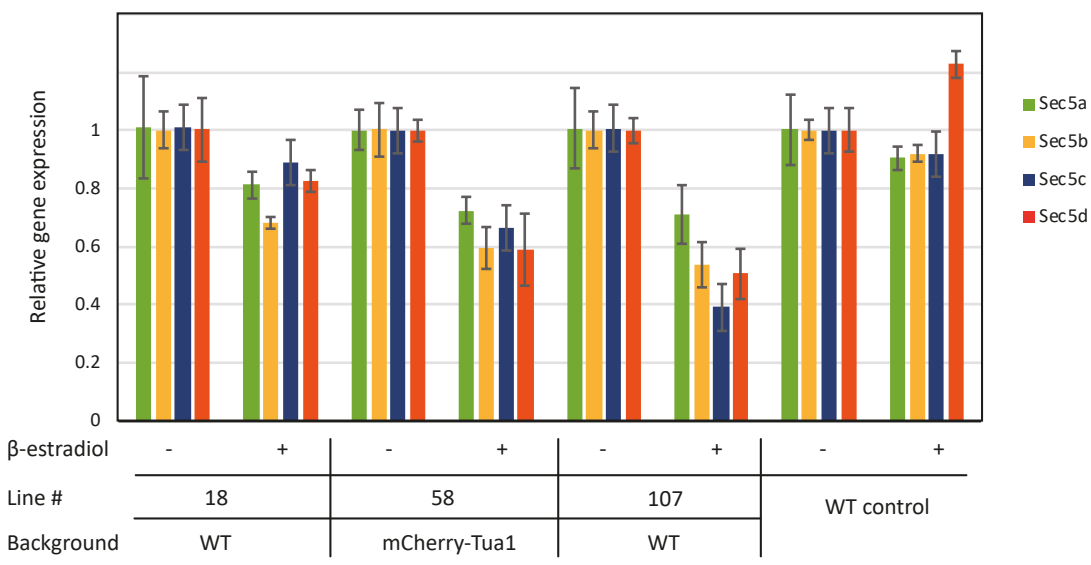

SUPPLEMENTARY FIGURE S3. Generation and verification of Sec5RNAi P. patens lines. (A) Schematic representation of the Sec5 silencing construct. PIG1bR/L: DNA fragments for homologous recombination into the PIG1 neutral locus. LexA:Pm35S: bacterial LexA operator fused to a CaMV minimal 35S promoter. Term: terminator. pKINID1a: promoter of a constitutively expressed $P$. patens gene. XVE: chimeric gene encoding a DNA-binding domain of the bacterial repressor LexA, transcriptional activation domain of VP16 an estrogen receptor domain. hygR: hygromicin resistance cassette driven by a modified CaMV35S promoter and terminator. Upon $\beta$-estradiol treatment, the XVE protein binds to the LexA operator and induces the expression of the Sec5-silencing fragment. Backbone of the construct is vector pGG626 (Nakaoka et al., 2012). (B) Verification of Sec5RNAi lines by quantitative RT-PCR. Relative expression of P. patens $\operatorname{Sec} 5 a, \operatorname{Sec} 5 b, \operatorname{Sec} 5 c$ and $\operatorname{Sec} 5 d$ in independent Sec5RNAi moss lines two days after growth on medium with and without $\beta$-estradiol. Transcript levels were normalized to PpEF1a transcript levels and expressed as mean fold changes ( \pm standard deviation) relative to the transcript level in non-induced moss tissue (set at 1 ).

SUPPLEMENTARY TABLE S1. Gene IDs.

\begin{tabular}{ll}
\hline Gene & Code \\
\hline PpSec5a & Pp1s41_289; Pp3c5_4060 \\
\hline PpSec5b & Pp1s38_295; Pp3c1_7430 \\
\hline PpSec5c & Pp1s18_342; Pp3c24_1100 \\
\hline PpSec5d & Pp1s11_129; Pp3c11_13110 \\
\hdashline AtSec5a & AT1G76850 \\
\hdashline AtSec5b & AT1G21170 \\
\hdashline StSec5-1 & Sotub04g023510 \\
\hdashline StSec5-2 & Sotub12g024810 \\
\hdashline ScSec5 & P89102 \\
\hline
\end{tabular}


SUPPLEMENTARY TABLE S2. Primers used in this study.

\begin{tabular}{|c|c|c|c|}
\hline Primer & Use & Sequence $\left(5^{\prime}>3^{\prime}\right)$ & $\mathbf{N r}$ \\
\hline Sec5a-F & $\begin{array}{l}\text { Cloning of Sec5a coding } \\
\text { sequence for } \mathrm{Y} 2 \mathrm{H}\end{array}$ & CACCATGAGGGACGACGACGAG & 2857 \\
\hline Sec5a-R & $\begin{array}{l}\text { Cloning of Sec5a coding } \\
\text { sequence for } \mathrm{Y} 2 \mathrm{H}\end{array}$ & CTAACCATCATGGAAGAGTCTTGGAAC & EO57 \\
\hline Sec5b-F & $\begin{array}{l}\text { Cloning of Sec } 5 \mathrm{~b} \text { coding } \\
\text { sequence for } \mathrm{Y} 2 \mathrm{H}\end{array}$ & CACCATGGCGATGCGACATGACG & 2855 \\
\hline Sec5b-R & $\begin{array}{l}\text { Cloning of Sec } 5 \mathrm{~b} \text { coding } \\
\text { sequence for } \mathrm{Y} 2 \mathrm{H}\end{array}$ & TTATCTCAAGTCATCGTTATCTACATAGGC & EO58 \\
\hline $\operatorname{Sec} 5 c-\mathrm{F}$ & $\begin{array}{l}\text { Cloning of Sec } 5 \mathrm{c} \text { coding } \\
\text { sequence for } \mathrm{Y} 2 \mathrm{H}\end{array}$ & CACCATGTCGACAGCGGCCTCAG & 2852 \\
\hline $\operatorname{Sec} 5 c-R$ & $\begin{array}{l}\text { Cloning of Sec } 5 \mathrm{c} \text { coding } \\
\text { sequence for } \mathrm{Y} 2 \mathrm{H}\end{array}$ & CTATGACTCCGGGATGGGAGG & EO59 \\
\hline Sec5d-F & $\begin{array}{l}\text { Cloning of Sec } 5 \mathrm{~d} \text { coding } \\
\text { sequence for } \mathrm{Y} 2 \mathrm{H}\end{array}$ & CACCATGGCGACGCGGGCGCC & 2860 \\
\hline Sec5d-R & $\begin{array}{l}\text { Cloning of Sec } 5 \mathrm{~d} \text { coding } \\
\text { sequence for } \mathrm{Y} 2 \mathrm{H}\end{array}$ & CTATCTCAACTCATCGTTATCTACATAAATTTGAGG & EO60 \\
\hline AVR1-F & AVR1 cloning & ATGTTCGACCACGACAAGG & 2072 \\
\hline Start-myc-F & Myc-Cer-AVR1/AT cloning & CACCGCAGAATGGGGTTAATTAACGGTGAAC & EO80 \\
\hline Myc-cer-R & Myc-Cer-AVR1/AT cloning & $\begin{array}{l}\text { CAGCTCCTCGCCCTTGCTCACGCTACC- } \\
\text { GTTCAAGTCTTCCTC }\end{array}$ & EO81 \\
\hline Cer-myc-F & Myc-Cer-AVR1/DT cloning & $\begin{array}{l}\text { GAGGAAGACTTGAACGGTAGCGTGAGCAAGGGC- } \\
\text { GAGGAGCTG }\end{array}$ & EO82 \\
\hline Cer-AVR1-R & Myc-Cer-AVR1/DT cloning & $\begin{array}{l}\text { GGAACCTTGTCGTGGTCGAACTTGTACAGCTCGTC- } \\
\text { CATGCC }\end{array}$ & EO83 \\
\hline AVR1-cer-F & Myc-Cer-AVR1/ $\Delta \mathrm{T}$ cloning & $\begin{array}{l}\text { GGCATGGACGAGCTGTACAAGTTCGACCACGA- } \\
\text { CAAGGTTCC }\end{array}$ & EO84 \\
\hline AVR1 $\Delta T$-R-wistop & Myc-Cer-AVR1 cloning & TTACCTCCACTTCACAGCCCGTGCC & EO85 \\
\hline AVR1-R-wistop & Myc-Cer-AVR1 $\Delta$ T cloning & TTAAAATGGTACCACAACATGTCC & EO86 \\
\hline PIG1-locus-R & Genotyping AVR1 lines & CCCTCTGCCGACCCATATTTC & EO114 \\
\hline AVR1-koz-F & $\begin{array}{l}\text { Genotyping AVR1 lines and } \\
\text { cloning AVR1/ } \triangle T-m y c\end{array}$ & CACCGCAGAATGTTCGACCACG & EO117 \\
\hline 10 myc-R & Cloning of AVR1/ $\Delta \mathrm{T}-\mathrm{myc}$ & GAAATTCGAGCCTAAGCACCGTTC & EO119 \\
\hline AVR1-F-gt & Genotyping AVR1 lines & ATGGGGTTAATTAACGGTGAACAAAAGC & EO120 \\
\hline AVR1-R-gt & Genotyping AVR1 lines & AAATGGTACCACAACATGTCCACCAAG & E0121 \\
\hline T35S-F & Genotyping AVR1 lines & TGCTAAGGCAGGGTTGGTTACG & JK130 \\
\hline AVR1 $1 \mathrm{~T}-\mathrm{R}-\mathrm{gt}$ & Genotyping AVR1 lines & CCTCCACTTCACAGCCCGTG & EO122 \\
\hline PpSEC5a_RNAi_F_Xhol & Cloning Sec5RNAi construct & CACCCTCGAGCCACCAAATCAGCCGCCT & EO49 \\
\hline PpSEC5a_RNAi_R_Sall & Cloning Sec5RNAi construct & AAAGTCGACCTCTGCTTGATCAACTCCACTCC & EO50 \\
\hline PpSEC5b_RNAi_F_Xhol & Cloning Sec5RNAi construct & CACCCTCGAGAGCAGGCAGCGAGGGAGA & EO51 \\
\hline PpSEC5b_RNAi_R_Sall & Cloning Sec5RNAi construct & AAAGTCGACTTCCAGGTCGCAGGCTCG & EO52 \\
\hline PpSEC5c_RNAi_F_Xhol & Cloning Sec5RNAi construct & CACCCTCGAGCCTGCAAAAACACCATTGACGAT & EO53 \\
\hline PpSEC5c_RNAi_R_Sall & Cloning Sec5RNAi construct & AAAGTCGACGAAAGCTCCAGATGAGGATCCTC & EO54 \\
\hline
\end{tabular}


SUPPLEMENTARY TABLE S2. (Continued)

\begin{tabular}{|c|c|c|c|}
\hline Primer & Use & Sequence $\left(5^{\prime}>3^{\prime}\right)$ & $\mathbf{N r}$ \\
\hline PpSEC5d_RNAi_F_Xhol & Cloning Sec5RNAi construct & CACCCTCGAGGAAGACGACGAAGAAGAGGAGC & EO55 \\
\hline PpSEC5d_RNAi_R_Sall & Cloning Sec5RNAi construct & AAAGTCGACGTTTCTCGAAGCTCCCGCA & EO56 \\
\hline PpSec5a_qPCR_F & qRT-PCR Sec5RNAi lines & CGGTCAAACTTTCCGAGTACG & 2772 \\
\hline PpSec5a_qPCR_R & qRT-PCR Sec5RNAi lines & TATGCCTCCATCACTGCCAAAT & 2773 \\
\hline PpSec5b_qPCR_F & qRT-PCR Sec5RNAi lines & GGGGCAACGCCTATGTAGA & 2768 \\
\hline PpSec5b_qPCR_R & qRT-PCR Sec5RNAi lines & ACACAGCCAGCTATTGCCTT & 2769 \\
\hline PpSec5c_qPCR_F & qRT-PCR Sec5RNAi lines & ATGTCACAGGAGGAAATACAGG & EO164 \\
\hline PpSec5c_qPCR_R & qRT-PCR Sec5RNAi lines & GTTTGGAAGGTAGTCTGCAATGA & EO165 \\
\hline PpSec5d_qPCR_F & qRT-PCR Sec5RNAi lines & CTTCCGTGGCAACCCTCAAA & 2770 \\
\hline PpSec5d_qPCR_R & qRT-PCR Sec5RNAi lines & CCCTGTGAGGCCTTCAATTCT & 2771 \\
\hline PpEF1a_F & qRT-PCR Sec5RNAi lines & AATCATACATTTCACCTCGCC & 2764 \\
\hline PpEF1a_R & qRT-PCR Sec5RNAi lines & GATCAGTGGGTAGAAGTGAC & 2765 \\
\hline
\end{tabular}

SUPPLEMENTARY TABLE S3. Plasmids used in this study.

\begin{tabular}{|c|c|c|}
\hline Plasmid & Usage & Source \\
\hline pENTR-D-TOPO-AVR1 & Cloning of AVR1 inducible expression vector & Du et al., 2015a \\
\hline pENTR-D-TOPO-A-L & Cloning of AVR1 inducible expression vector & Du et al., 2015a \\
\hline pENTR-D-TOPO-AVR1 $\Delta T$ & Cloning of AVR1 inducible expression vector & Du et al., 2015a \\
\hline pENTR-D-TOPO-myc-Cer-AVR1 & Cloning of AVR1 inducible expression vector & This study \\
\hline pENTR-D-TOPO-myc-Cer-AVR1 $\Delta T$ & Cloning of AVR1 inducible expression vector & This study \\
\hline pGWB20-AVR1-10myc & Cloning of AVR1 inducible expression vector & Du et al., 2015a \\
\hline pGWB20-AVR1 $\Delta \mathrm{T}-10$ myс & Cloning of AVR1 inducible expression vector & This study \\
\hline pENTR-D-TOPO-AVR1-10myc & Cloning of AVR1 inducible expression vector & This study \\
\hline pENTR-D-TOPO-AVR1 $\Delta T-10 m y c$ & Cloning of AVR1 inducible expression vector & This study \\
\hline pPGX8 & $\beta$-estradiol inducible expression & Kubo et al., 2013 \\
\hline pPGX8-AVR1-myc & $\beta$-estradiol inducible AVR1 expression & This study \\
\hline pPGX8-AVR1 $\Delta \mathrm{T}-\mathrm{myc}$ & $\beta$-estradiol inducible AVR1 $\Delta$ T expression & This study \\
\hline pPGX8-myc-Cer-AVR1 & $\beta$-estradiol inducible AVR1 expression & This study \\
\hline pPGX8-myc-Cer-AVR1 $\Delta \mathrm{T}$ & $\beta$-estradiol inducible AVR1 $\Delta T$ expression & This study \\
\hline pENTR-D-TOPO-Sec5a & Cloning for yeast-two-hybrid assay & This study \\
\hline pENTR-D-TOPO-Sec5b & Cloning for yeast-two-hybrid assay & This study \\
\hline pENTR-D-TOPO-Sec5c & Cloning for yeast-two-hybrid assay & This study \\
\hline pENTR-D-TOPO-Sec5d & Cloning for yeast-two-hybrid assay & This study \\
\hline pDEST22-Sec5a & Yeast-two-hybrid assay & This study \\
\hline pDEST22-Sec5b & Yeast-two-hybrid assay & This study \\
\hline pDEST22-Sec5c & Yeast-two-hybrid assay & This study \\
\hline
\end{tabular}


SUPPLEMENTARY TABLE S3. (Continued)

\begin{tabular}{lll}
\hline Plasmid & Usage & Source \\
\hline pDEST22-Sec5d & Yeast-two-hybrid assay & This study \\
\hline pDEST32-AVR1 & Yeast-two-hybrid assay & Du et al., 2015a \\
\hline pDEST32-AVR1 $\Delta T$ & Yeast-two-hybrid assay & Du et al., 2015a \\
\hline pDEST32-A-L & Yeast-two-hybrid assay & Du et al., 2015a \\
\hline pDEST32-myc-Cer-AVR1 & Yeast-two-hybrid assay & This study \\
\hline pDEST32-myc-Cer-AVR1 $\triangle T$ & Yeast-two-hybrid assay & This study \\
\hline pGG626 & Induced gene silencing in $P$. patens & Nakaoka et al., 2012 \\
\hline pENTR-D-TOPO-Sec5RNAi & Gene silencing of all four $P$. patens homologs & This study \\
\hline pGG626-Sec5RNAi & Gene silencing of all four $P$. patens homologs & This study \\
\hline
\end{tabular}

SUPPLEMENTARY TABLE S4 - P. patens strains used in this study.

\begin{tabular}{llll}
\hline P. patens line & Representative clone(s) & Background strain & Reference \\
\hline AVR1-myc/Sec5b-GFP & $66,127,212$ & Sec5b-GFP & This study \\
\hline AVR1-myc/Sec5d-GFP & $147,201,208$ & Sec5d-GFP & This study \\
\hline AVR1 $\Delta$ T-myc/Sec5b-GFP & $5,11,224$ & Sec5b-GFP & This study \\
\hline AVR1 $\Delta$ T-myc/Sec5d-GFP & $27,126,148$ & Sec5d-GFP & This study \\
\hline myc-Cer-AVR1/Sec5b-GFP & $119,125,174$ & Sec5b-GFP & This study \\
\hline myc-Cer-AVR1/Sec5d-GFP & $9,11,26$ & Sec5d-GFP & This study \\
\hline myc-Cer-AVR1 $\Delta$ T/Sec5b-GFP & $27,235,422$ & Sec5b-GFP & This study \\
\hline myc-Cer-AVR1 $\Delta$ T/Sec5d-GFP & $3,15,18$ & Sec5d-GFP & This study \\
\hdashline Sec6RNAi & 6 & Sec6-GFP/Scamp4-mCherry & Tang et al., 2019 \\
\hline Sec5RNAi & 18,107 & WT, Gransden isolate & This study \\
\hline Sec5RNAi & 58 & mCherry-Tua1 & This study \\
\hline
\end{tabular}




\section{Chapter 6}

General discussion 


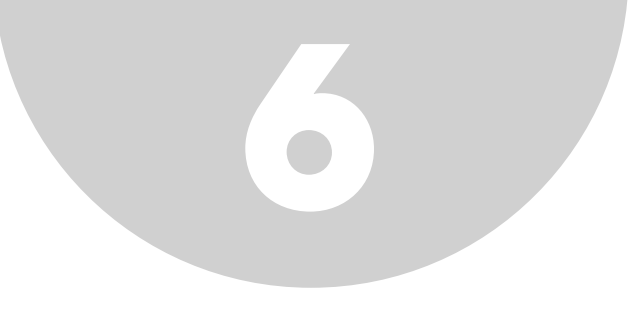

\section{General discussion}

When plants are challenged by pathogens, plant defence responses are rapidly activated and targeted to the site of infection including the secretion of defence-related materials. The central goal of this thesis is to understand this polarized exocytosis with a focus on the role of the exocyst complex in plant immunity. We demonstrated that multiple $N$. benthamiana exocyst subunits play a role in defence against unrelated pathogens (Chapter 2; Du et al., 2018a) and revealed that several exocyst subunits accumulate at infection sites in $P$. patens (Chapter 4). In addition, we showed that the Phytophthora infestans effector AVR1 affects exocyst functioning in P. patens by interacting with exocyst subunit Sec5 (Chapter 5). In this final chapter, I reflect on the main results and discuss them in a broader perspective. I start by discussing the challenges and opportunities of imaging plant-microbe interactions followed by a reflection on the advantages and disadvantages of the Physcomitrella-Phytophthora model system that we developed to visualize cellular plant defence processes (Chapter 3; Overdijk et al., 2016). Subsequently, the knowledge on signalling pathways that give rise to polarization of exocytosis in well-studied model plant systems, such as tip growth, will be summarized and integrated in a model for the regulation of polarized exocytosis in cells attacked by pathogens. Next, I zoom in on one of the components involved in polarizing exocytosis, namely the exocyst complex. I will discuss the role of the exocyst complex in plant immunity as well as the proposed mode of action of pathogen effectors that target exocyst subunits.

\section{Imaging plant-microbe interactions}

Since the 1940s, phytopathologists have made extensive progress in understanding the genetics of plant immunity and in the last decades they have broadened the scope of their research to understand the cellular and molecular mechanisms that coordinate plant immune signalling. Plant defence responses are local and the visualization of real-time molecular dynamics of immune signalling is essential to, for example, understand signal perception, subsequent activation and recruitment of defence responses (Deeks \& SanchezRodriguez, 2016). The opportunity to generate transgenic plants expressing fluorescentlytagged proteins allows for live-cell imaging of the localization and dynamics of plant proteins. Imaging these fluorescently-tagged proteins in plants is challenged by the presence of intrinsic autofluorescence, e.g. from chlorophyll in plastids or from lignin and other phenolic compounds in the plant cell wall (Shaw \& Ehrhardt, 2013; Zhu et al., 2018). Combined with another problem, namely light scattering caused by plant cell walls and the vacuole, this limits deep-tissue imaging and imaging of proteins with low expression levels (Grossmann et al., 2018). These issues also apply to the imaging of plants that interact with microbes. Highly autofluorescent material is deposited at infection sites (Vleeshouwers et al., 2000; Overdijk et al., 2016) and interactions with microbes often take place in the subepidermal 
layer requiring deep imaging. Ideally, plant-microbe interactions are followed over time. In order to capture the molecular dynamics of these interactions, high-resolution imaging is ideally combined with live-cell imaging. Therefore, non-invasive imaging is preferred over invasive sectioning. Recent advances in imaging techniques, setups and novel model systems are able to reduce these imaging issues and allow in vivo high-resolution tracking of cellular dynamics.

There are several ways to eliminate the intrinsic autofluorescence of plants and specifically that of defence-related compounds. Some modern confocal systems have the option for spectral unmixing, which is a computational technique that differentiates between emission spectra to separate fluorescent signals with different spectra (Grossmann et al., 2018). More recently, time-gated confocal microscopy is described as a way to efficiently eliminate autofluorescence (Chapter 4; Kodama, 2016). This technique makes use of ultrashort pulsed lasers and detectors that have a photon-counting ability. In general, autofluorescence has a very short lifetime (picosecond range) compared to the lifetime of fluorescent proteins (nanosecond range) (Grossmann et al., 2018). By omitting the fluorescence that is detectable in the first picoseconds after excitation, the undesired autofluorescence is successfully eliminated (Kodama, 2016). Autofluorescence can also be diminished by clearing techniques, but these are often not compatible with live-cell imaging (Grossmann et al., 2018).

Several approaches help to avoid the issues encountered with deep-tissue imaging. One way is to use two-photon excitation microscopy which circumvents the light-scattering effects of plant cell walls. This technique uses two low-energy photons that only excite the target fluorophore in the focal plane (Feijo \& Moreno, 2004). Another technique is light sheet fluorescence microscopy that facilitates deep-tissue high-resolution imaging of whole organisms in 3D over a long period of time, a major challenge however is to maintain proper physiological conditions over time (Grossmann et al., 2018). In addition to these microscopy techniques, the availability of cell type-specific promoters in Arabidopsis thaliana has improved deep-tissue imaging, since they eliminate signals from surrounding tissue (Grossmann et al., 2018). The combined use of two-photon excitation microscopy and these cell type-specific promoters enabled live-cell imaging and cell lineage tracing in developing $A$. thaliana embryos (Gooh et al., 2015). To my knowledge, neither two-photon excitation microscopy nor light sheet fluorescence microscopy have been applied to image plant-microbe interactions yet. These powerful tools could be for example used to visualize the dynamics of proteins during haustoria formation in the mesophyll layer of plants. Another way to avoid the disadvantages of deep-tissue imaging in plants, is to move to plants with a less complex body plan and use tissues with one or two cell layers for imaging.

\section{Physcomitrella as model system for imaging cellular defence processes}

The moss Physcomitrella patens is such a plant with a relatively simple body plan consisting of tissues that are a single-cell layer thick, making it ideal for high-resolution microscopy (Knight et al., 2009). In addition to these ideal morphological properties for microscopy, 
it is easily cultured and stable transgenic plants can be generated relatively fast (Knight et al., 2009). P. patens has a convenient toolbox for gene modification. Its homologous recombination ability allows direct integration of fluorescent tags in the endogenous locus in the genome, guaranteeing endogenous timing and levels of expression of the tagged genes (Nishiyama et al., 2000). Also CRISPR/Cas-mediated genome editing is readily available to efficiently generate knock outs (Lopez-Obando et al., 2016; Yi \& Goshima, 2019).

$P$. patens is more and more used to study plant immunity, often from an evolutionary perspective (Ponce de Leon \& Montesano, 2017; Carella \& Schornack, 2018). These studies showed that $P$. patens is susceptible to several pathogens and that these pathogens induced defence responses that are similar to those found in seed plants, including cell death, the upregulation of defence-related genes and reactive oxygen species (ROS) production (Ponce de Leon \& Montesano, 2017). Bressendorff et al. (2016) showed that chitin-induced defence signalling in P. patens activates similar MAP kinase cascades as in A. thaliana.

In addition to the pathosystems that have been described for P. patens (Ponce de Leon \& Montesano, 2017), we developed a novel pathosystem using Phytophthora pathogens and demonstrated its value for imaging cellular defence processes (Chapter 3; Overdijk et al., 2016). Out of four tested Phytophthora species, P. infestans and P. capsici were able to penetrate P.patens protonema cells or induce the formation of papilla-like structures halting hyphal penetration. The presence of papilla-like structures in P. patens and their similarity to those found in seed plants (Oliver et al., 2009; Lehtonen et al., 2012b; Overdijk et al., 2016; Yan et al., 2018), indicates that papilla formation is highly conserved. We developed a special imaging set-up using coverglass-bottom microwell dishes that allow for highresolution live-cell imaging of protonema cells. P. patens protonema tissue covered with a thin layer of agar was grown in these dishes for about a week, subsequently inoculated with zoospores of Phytophthora and the first penetration attempts were observed a few hours later. The power of this set-up was demonstrated by visualizing the rapid and local accumulation of actin filaments at infection sites, another common defence response observed in seed plants (Hardham et al., 2007). Moreover, combined with time-gated confocal microscopy to eliminate the autofluorescence of defence-related compounds, this system allowed us to show that $P$. patens exocyst subunits accumulate at infection sites (Chapter 4) and that $P$. infestans RXLR effector AVR1 does not alter the localization of its target protein Sec5 (Chapter 5).

Besides the evident advantages of the Physcomitrella-Phytophthora system, it also comes with some constraints. Although it is an excellent system to study cellular defence processes, its single-cell layered morphology makes it less suitable for testing infection efficiency on a tissue level for instance by measuring lesion sizes. We never observed the formation of haustoria inside P. patens tissue (Overdijk et al., 2016). In Solanaceous host plants, $P$. infestans and $P$. capsici first penetrate the epidermal cell layer and subsequently form haustoria inside the mesophyll layer below (Judelson \& Blanco, 2005). The absence of multi-cell layered tissue could explain the lack of haustoria in P. patens. Therefore, it 
could be argued that $P$. patens is not a true host for $P$. infestans or $P$. capsici and that this pathosystem is only suitable to study initial infection and defence processes such as cell wall penetration, papilla formation and invasive hyphal growth. To study haustoria formation in an evolutionary perspective, Carella et al. (2018) recently developed a model system using Phytophthora palmivora and the liverwort Marchantia polymorpha, which tissue consists of multiple cell layers. They showed that $P$. palmivora establishes intracellular haustoria-like infection structures that accumulate components of the host cellular trafficking machinery, furthermore illustrating that the intracellular accommodation of filamentous microbes is an ancient plant trait.

Another limitation of the Physcomitrella-Phytophthora system concerns the used imaging set-up. We observed that the delicate $P$. patens protonema cells are sensitive to perturbations and that this influences their defence capacity, for example the ability to form papilla-like structures (Overdijk et al., 2016). This was the main reason for growing $P$. patens in special cover glass-bottom microwell dishes, minimizing manipulation prior to imaging. However, this set-up made it difficult to get the Phytophthora zoospores, and hence infection events, within the optimal range for confocal microscopy, namely between the coverslip and the protonema-containing agar, without disturbing the protonemal cells. In addition, we have attempted to image infection processes over long time periods (hours), but failed mainly because infection readily arrested upon imaging.

Despite a high concentration of Phytophthora zoospores used in the infection assays, penetration events were rare and not synchronized, making imaging infection events laborious and time consuming. It is known from $A$. thaliana that mechanical stimulation with a microneedle results in rapid reorganization of subcellular components, including actin filaments, ER and peroxisomes, mimicking the response to a pathogen attack (Hardham et al., 2008). My own observations confirmed such a response for P. patens protonema cells. After stimulation with a microneedle, rapid actin filament accumulation was observed at the contact sites, which could be used in future experiments to mimic pathogen attack (Figure 1, unpublished data). In addition, elicitors could be used to mimic pathogen presence. Sassmann et al. used a mix of plant cell wall hydrolysing enzymes supplemented with chitin oligomers to induce accumulation of fluorescently-labelled AtFormin4, thereby successfully imitating a fungal infection in A. thaliana (Sassmann et al., 2018).

A

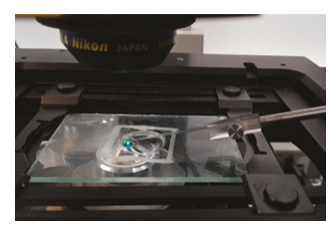

B Before touch

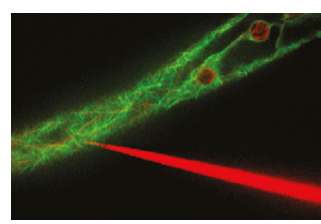

18 sec after touch

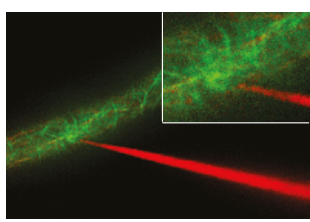

$120 \mathrm{sec}$ after touch

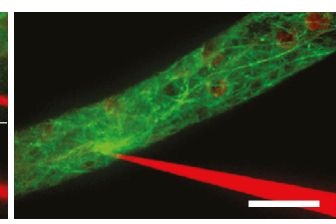

FIGURE 1. Rapid actin accumulation upon mechanical stimulation with a glass microneedle. A) Imaging set-up on a Spinning disk confocal microscope. B) P. patens cell in which the actin cytoskeleton is visualised by LifeAct-GFP and the microtubule cytoskeleton by mCherry-Tua1 before and after touch. First two images are single confocal planes, the last one represents a maximum z-projection of $7 \mu \mathrm{m}$. Scale bar represents $10 \mu \mathrm{m}$. 
To further explore the feasibility of the Physcomitrella-Phytophthora pathosystem as a model system for studying plant-pathogen interactions, I performed infections assays on P. patens grown in polydimethylsiloxane (PDMS)-based microfluidic growth chambers (unpublished data). These microfluidic devices are miniature PDMS channel systems in a coverslip-bottom dish filled with liquid medium (Bascom et al., 2016). P. patens can be easily cultured in these chambers. Because the plant tissue is restricted to a $30-\mu \mathrm{m}$-deep chamber above the coverslip, the majority of the tissue is within the optimal imaging range for confocal microscopy (Bascom et al., 2016). In addition, microfluidic devices make it easy to precisely control the microenvironment, e.g. by adding fluorescent dyes or drugs (Grossmann et al., 2018). The use of fluorescent indicators allows non-invasive measurements of various biochemical and biophysical parameters, e.g. local calcium levels, $\mathrm{pH}$ and ROS. As stated by Grossmann et al. (2018), 'microfluidic devices are at the forefront of innovation for culturing, imaging and manipulating plants in controlled environments'. Microfluidic devices have been for example used to image $A$. thaliana roots over a long time or facilitate high-resolution phenotyping of $A$. thaliana seedlings (Jiang et al., 2014; Grossmann et al., 2018). Regarding plants interacting with microbes, nematode feeding and Phytophthora sojae colonization of $A$. thaliana roots has been visualized in microfluidic devices, as well as the formation of microbial biofilms along specific root zones and the dynamics of root colonization by beneficial bacteria (Parashar \& Pandey, 2011; Massalha et al., 2017; Aufrecht et al., 2018). Upon inoculation with P. capsici zoospores, I observed efficient papillae formation in $P$. patens tissue grown in these microfluidic devises (unpublished data). The liquid medium inside the microfluidic chambers is ideal for the Phytophthora zoospores to swim to protonema cells and develop infection sites close to the coverslip. Together, this shows that microfluidic devices are promising tools to further optimize the Physcomitrella-Phytophthora imaging set-up.

\section{How is polarized exocytosis during plant defence orchestrated?}

Polarized secretion is a general and essential part of the plant's basal defence repertoire (Gu et al., 2017). Upon contact with a fungal or oomycete pathogen, the plant cell targets vesicles containing amongst others antimicrobial compounds and cell wall material to infection sites (Park et al., 2018). This is a local and precise response that requires tight regulation. Which molecular players orchestrate this polarized secretion to infection sites? Well-known conserved master regulators of polarity in plants are the Rho of Plant (ROP) proteins, also known as RACs, which are a plant-specific family of Rho small GTPases (Feiguelman et al., 2018). ROPs are involved in many developmental processes that depend on polarity including tip growth, cell shape and cytokinesis (Yang, 2008). They translate polarity into cellular responses, for example by regulating organization of the cytoskeleton and membrane trafficking. Could ROPs also be involved in regulating polarity and in particular in polarizing exocytosis during plant defence? 


\section{ROPs are involved in plant-microbe interactions}

Indeed, several studies indicate that ROPs function upon pathogen attack and during interaction with beneficial microbes (Figure 2). For example a ROP protein in rice, OsRAC1, participates in defence responses induced by pathogen/microbe-associated molecular patterns (PAMPs/MAMPs), including chitin (Akamatsu et al., 2013; Nagano et al., 2016). OsRAC1 activates lignin biosynthesis and RBOH-type NADPH oxidases that produce ROS, which function as second messengers in plant defence (Kawasaki et al., 2006; Wong et al., 2007; Torres, 2010). In barley, several ROPs, i.e. HvRACB, HvRAC1 and HvRAC3, act as susceptibility factors and are required for entry of the adapted powdery mildew fungus Blumeria graminis f.sp. hordei (Bgh) (Schultheiss et al., 2002; Schultheiss et al., 2003; Pathuri et al., 2008). The function of HvRACB includes the modulation of actin organization at the penetration attempt site (Opalski et al., 2005). However, in response to the nonadapted rice blast fungus Magnaporthe oryzae, HvRAC1 is involved in ROS production, callose deposition and increased resistance (Pathuri et al., 2008). In A. thaliana, AtROP6 is involved in salicylic acid (SA)-mediated defence responses (Poraty-Gavra et al., 2013). Plants expressing a dominant negative AtROP6 showed enhanced defence responses to the adapted powdery mildew fungus Golovinomyces orontii which is likely related to constitutive active SA-signalling. Surprisingly, these plants were more susceptible to the non-adapted powdery mildew fungus Bgh (Poraty-Gavra et al., 2013). Recently, Yang et al. showed that cotton GhRAC6 is involved in resistance against aphids (Yang et al., 2018). Finally, during the interaction with beneficial microbes, Medicago truncatula ROP10 and Lotus japonicus ROP6 are required for successful rhizobium-induced nodule formation (Ke et al., 2012; Lei et al., 2015; Wang et al., 2015; Rivero et al., 2017). Together, this indicates that ROP-mediated signalling plays a role in plant-microbe interactions. But what could be the function of ROPs during these interactions? Which downstream signalling pathways could ROPs regulate? And how is polarization during infection established? To examine these questions, I will explore the knowledge on plant cells that exhibit extremely polarized growth, namely tip growing root hairs and pollen tubes. In these cells, the growth machinery is targeted to a small surface of the cell, namely the tip, which is similar to the local secretion response induced by attempted pathogen penetration.

\section{Building polarity and activating ROP-signalling}

ROPs cycle between a GDP-bound inactive and GTP-bound active state (Figure 2). They are activated by plant specific GEFs (GDP/GTP Exchange Factors) that facilitate the release of GDP, and inactivated by GAPs (GTPase-Activating Proteins) that enhance GTP hydrolysis resulting in GDP-bound ROP (Berken \& Wittinghofer, 2008). Active GTP-ROPs are bound to the plasma membrane and localize in distinct plasma membrane domains (Feiguelman et al., 2018). A third group of ROP regulatory proteins, the RhoGDIs (Rho GDP Dissociation Inhibitors) remove inactive GDP-ROP from the plasma membrane and sequester them in the cytoplasm until their reactivation by GEFs (Boulter \& Garcia-Mata, 2010). A recent study in $A$. thaliana showed that two GEFs are involved in polarizing and initiating root hair formation (Denninger et al., 2019). AtGEF3 acts as a landmark at the future site of root hair bulging where it targets AtROP2 and positions the molecular machinery for growth. 


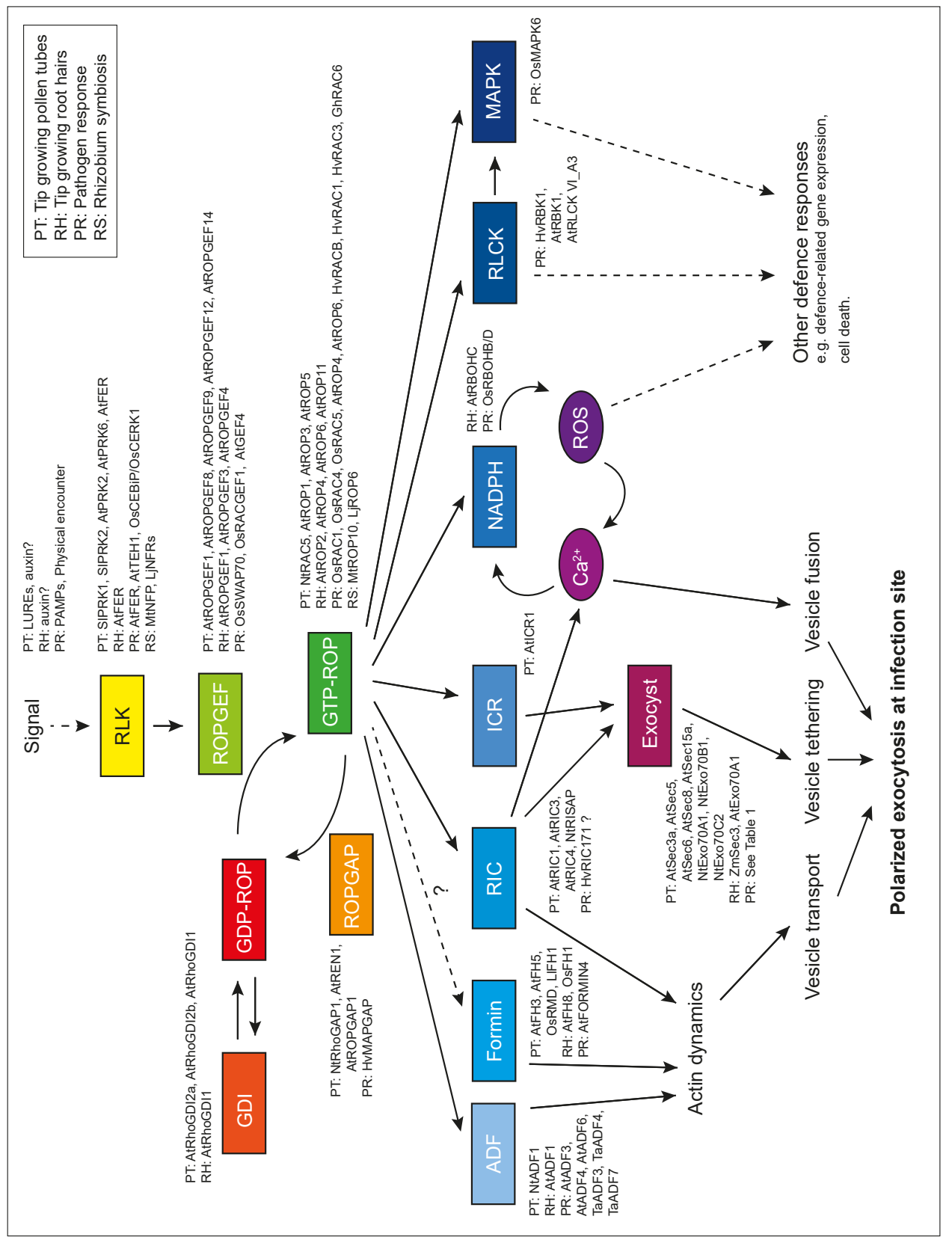

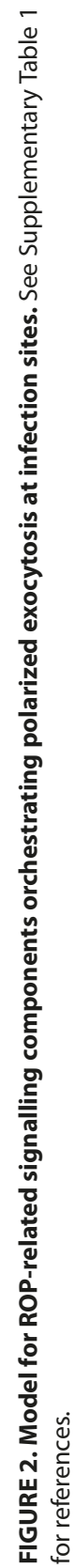


Subsequently, AtGEF4 is recruited to further facilitate tip growth. This positions GEFs as candidates for polarizing and activating ROP-signalling during plant defence. Indeed, several studies indicate that GEFs activate ROPs involved in plant immunity, for example the above mentioned OsRAC1 from rice is activated by GEFs OsRACGEF1 and OsSWAP70 (Yamaguchi et al., 2012; Akamatsu et al., 2013). Knockdown of OsRACGEF1 impairs the expression of defence-related genes and increases susceptibility to the rice blast fungus $M$. oryzae (Akamatsu et al., 2013). The next question is then how GEFs are activated themselves.

From tip growing cells it is known that Receptor-Like Kinases (RLKs) are the most upstream factors that activate ROP-signalling (Figure 2). RLKs are membrane-bound receptors that consist of an extracellular domain for ligand binding and an intracellular kinase domain that activates downstream signalling. Pollen tubes are attracted to ovules by small peptides called LUREs, which are recognized by RLKs that localize in the pollen tube tip and activate ROP-signalling (Takeuchi \& Higashiyama, 2016). In addition, auxin, which promotes tip growth in root hairs and pollen tubes, is hypothesised to initiate ROP-signalling through an RLK receptor during tip growth, but this receptor is not yet identified (Pan et al., 2015). For plant immunity, the recognition of PAMPs/MAMPs by receptors including RLKs, is an important step in the initiation of defence responses (Jones \& Dangl, 2006). The fungal cell wall component chitin is one of the best-characterized PAMPs. In rice, chitin-perception is mediated by the RLK OSCERK1 that forms a complex with chitin-binding protein OsCEBiP (Shimizu et al., 2010). Upon chitin binding, OsCERK1 activates ROP OsRAC1 via GEFs and this leads to lignin biosynthesis and ROS production (Wong et al., 2007; Akamatsu et al., 2013; Nagano et al., 2016). Chitin fragments are present throughout the invaded apoplast and not only around the local site of hyphal penetration. Therefore, chitin-perception itself will not likely lead to a locally targeted defence response in the plant cell. Is ROP-signalling included in the chitin-perception pathway to polarize this response? This would assume local accumulation of ROPs at infection sites, but as yet this has not been investigated. So far there is only one RLK found, namely OsCERK1, that links PAMP-perception with ROPsignalling. Whether or not other defence-related RLKs activate ROPs is still an open question.

Besides molecular signals, another external cue that could activate ROP-signalling upon pathogen attack is the physical encounter with the pathogen itself. Mechanical stimulation of an A. thaliana cell with a microneedle induced rapid subcellular reorganization including actin accumulation, thus mimicking a fungal or oomycete pathogen attack (Hardham et al., 2008). I have observed a similar behaviour in P. patens in which touching a protonema cell with a glass microneedle resulted in rapid actin accumulation (Figure 1, unpublished data). This indicates that physical cell wall stress is perceived and translated into a cellular response, possibly via ROP-signalling. The RLK FERONIA (AtFER), which is important for tip growth in root hair cells and directly interacts with ROPGEFs in A. thaliana (Duan et al., 2010), acts as a cell wall mechanosenor (Shih et al., 2014). A. thaliana mutants lacking FER were not able to properly respond to local touch or bending and were affected in their susceptibility to a powdery mildew fungus (Kessler et al., 2010; Shih et al., 2014). Moreover, another A. thaliana RLK, THESEUS1 (AtTHE1), which is a sensor for cell wall damage (Hofte, 2015), was found to regulate defence responses against the necrotrophic fungus $B$. cinerea via interacting 
with AtGEF4 (Qu et al., 2017). Together, this suggests that mechanical stress caused by the encounter with a pathogen itself is perceived by particular RLKs that activate ROP-mediated defence responses. Besides the mentioned cell wall sensing RLKs, it is also likely that there are proteins involved that sense membrane properties including membrane tension and curvature, for example mechanosensitive calcium channels that open upon mechanical touch and facilitate downstream signalling (Schoenaers et al., 2017).

\section{Translating ROP-activity into actin organization that facilitates vesicle trafficking}

Polarized secretion at sites of attempted penetration requires transport and accumulation of exocytotic vesicles containing defence-related components. Which processes are mediating and regulating this and what could be the role of activated ROPs herein? It is known that the actin cytoskeleton is involved in transport of defence-related components to the site of infection (Li \& Day, 2019). In a similar way in tip growing plants cells, a dynamic actin cytoskeleton is required for directional transport of vesicles and organelles to the tip (Ketelaar, 2013; Fu, 2015). Thick long actin cables facilitate vesicle targeting to and from the tip and fine filamentous actin in the tip apex mediates the accumulation of exocytotic vesicles (Ketelaar, 2013; Fu, 2015). The dynamics of the actin cytoskeleton are regulated by dozens of actin-binding proteins (Porter \& Day, 2016). ROPs play a role in regulating the activity of these actin-binding proteins, thereby translating polarity signals into actin organization and subsequent vesicle trafficking (Figure 2). ROP Interacting CRIB-containing proteins (RICs) are downstream effectors of active ROPs that regulate cytoskeleton dynamics (Yang, 2008). In pollen tubes for example, two counteracting RICpathways regulate actin dynamics: AtRIC4 promotes actin assembly, while AtRIC3 facilitates actin depolymerization by increasing calcium levels (Gu et al., 2005). Overexpression of either one of them resulted in depolarized pollen tube growth, suggesting that a tight balance between these two RIC pathways is essential for proper tip growth. Are there also RICs known that play a role in plant-microbe interactions? So far the only known one is barley HvRIC171, which is regulated by ROP HvRACB and accumulates at sites of fungal attack (Schultheiss et al., 2008). However, it remains to be studied whether HvRIC171 regulates actin dynamics during infection. Based on the knowledge from tip growing cells and the fact that the ROP-signalling pathway is activated upon pathogen recognition, it is tempting to speculate that there are two counteracting RICs that regulate actin dynamics during plant defence.

Other actin-binding proteins that could be involved in the ROP-mediated regulation of the actin cytoskeleton during infection are actin-depolymerizing factors (ADFs). ADFs enhance actin turnover by depolymerisation and filament severing (Maclver \& Hussey, 2002). In A. thaliana, AtADF1 plays a role in organizing the actin cytoskeleton during root hair growth (Dong et al., 2001). Since tobacco ROP NtRAC5 influences the activity of ADF1 in pollen tubes (Chen et al., 2003), it is plausible that ADFs are regulated by ROPs. Other A. thaliana ADFs include AtADF4 that facilitates PAMP/MAMP-triggered actin remodelling and callose deposition and plays a role in defence against the powdery mildew fungus $G$. orontii (Henty-Ridilla et al., 2014; Inada et al., 2016). The activity of AtADF6 was found to be responsible for a reduced accumulation of resistance protein AtRPW8.2 to extrahaustorial 
membranes and AtADF3 was involved in resistance against aphids (Wang et al., 2009; Mondal et al., 2018) . Moreover in wheat, three TaADFs contribute to resistance against the stripe rust fungus Puccinia striiformis (Fu et al., 2014; Tang et al., 2016; Zhang et al., 2017). Together, this suggests that ADFs play a role in regulating actin dynamics during plant defence. Whether ROPs regulate ADF activity upon pathogen attack still needs to be studied. Another actin-binding protein that is involved in actin turnover is profilin (Porter \& Day, 2016). Profilin might play a role in plant immunity since immunocytochemical staining of parsley cells infected with $P$. infestans revealed profilin accumulation at sites of attempted penetration (Schutz et al., 2006). Since profilin binds to actin monomers, this accumulation could also reflect cytoplasmic aggregation and not be related to a function during plant defence. However, a recent study in A. thaliana suggests that one of the profilins, AtPRF3, regulates PAMP-triggered defence responses (Sun et al., 2018). To date, no link between ROP-signalling and profilins has been reported, also not for Rho GTPases outside the plant kingdom, making it unlikely that ROPs are involved in regulating profilin activity.

Another group of actin-binding proteins that recently emerged as regulators of plant defence are the formins. Formins stimulate actin nucleation and polymerization and are important for tip growth (van Gisbergen \& Bezanilla, 2013; Schoenaers et al., 2017). Sassmann et al. (2018) showed that AtFORMIN4 accumulates at fungal attack sites and contributes to local actin dynamics required for pre-invasive defence responses. Formins are likely involved in building the actin cytoskeleton network that facilitates vesicle trafficking to the infection site and might form a link with the cell wall. Unlike in animals where Rho GTPases directly activate formins, such a relation has not yet been found for ROPs (Schoenaers et al., 2017). Finally, there could be another actin-binding protein family involved in ROP-mediated regulation of actin dynamics during infection. The ActinRelated Protein 2/3 (Arp2/3) complex promotes actin filament nucleation and branching (Hussey et al., 2006). During diffuse growth in trichomes and pavement cells, ROPs interact with the SCAR/WAVE complex that activates Arp2/3 (Feiguelman et al., 2018). Although evidence is limited, the Arp2/3 complex is suggested to regulate the formation of fine filamentous actin in root hairs thereby influencing root hair polarity (Schoenaers et al., 2017). A role for the Arp $2 / 3$ complex in plant defence has so far not been described.

\section{Vesicle tethering and fusion by the exocyst complex and calcium}

Once the exocytotic vesicle arrives at the site of infection facilitated by the actin cytoskeleton, it needs to be tethered and subsequently fused with the plasma membrane. The exocyst complex mediates this tethering to the plasma membrane (Ravikumar et al., 2017). The exocyst complex is as such involved in many developmental processes that depend on polarized secretion, including tip growth (Zarsky et al., 2013). For example exocyst subunits AtSec3a, AtSec5, AtSec6, AtSec8 and AtSec15a are required for pollen tube growth in $A$. thaliana and several $\mathrm{NtExo70}$ family members play a role in pollen tube growth in tobacco (Hala et al., 2008; Bloch et al., 2016; Sekeres et al., 2017). Is there a link between ROP-signalling and the exocyst complex? In yeast, active Rho GTPases directly interact with exocyst subunits $\mathrm{Sec} 3$ and Exo70, which is thought to regulate the localization and function of the exocyst complex (Mei \& Guo, 2018). However, in plants, such direct 
interaction has not been found. Instead, AtICR1 (Interactor of Constitutive Active ROP) which is activated by ROPs, was found to bind exocyst subunit Sec3a in A. thaliana (Lavy et al., 2007). AtICR1 localizes to the tip of growing pollen tubes and is involved in mediating the polar localization of AtROP1 in the tip (Li et al., 2008). In addition to the ICR1 link between ROP and the exocyst complex, Hong et al. (2016) recently showed that AtRIC7, which is activated by AtROP2, negatively regulates stomatal opening by inhibiting exocyst subunit AtExo70B1 via a direct interaction. As will be described later, there is increasing evidence that the exocyst is also involved in plant immunity. Together, this suggests that the exocyst is a downstream factor of the ROP-mediated signalling pathway and that this pathway could be involved in plant immunity regulating the final steps of polarized secretion of defence-related compounds.

After tethering of the vesicle to the plasma membrane, it needs to fuse and it is known that ROP-induced calcium levels facilitate this vesicle fusion (Figure 2; Schoenaers et al., 2017). Calcium is a versatile second messenger and an increase in calcium levels is one of first responses observed upon pathogen recognition (Aldon et al., 2018). In root hairs, calcium affects actin dynamics by regulating ADF activity and this is suggested to contribute to the formation of fine filamentous actin in the tip apex that could facilitate trapping of vesicles in the tip (Ketelaar, 2013). Besides regulating vesicle fusion and actin dynamics, calcium is part of a positive feedback loop with ROS producing NADPH oxidases that are activated by ROPs (Schoenaers et al., 2017).

\section{Downstream defence-signalling activated by the ROP-pathway}

Receptor-Like Cytoplasmic Kinases (RLCKs) and Mitogen-Activated Protein Kinases (MAPKs) are crucial in transducing downstream signalling of activated PAMP-recognizing receptors, the RLKs (Cui et al., 2018). In addition, they are suggested to be activated by the ROP-signalling pathway. RLCKs lack an extracellular domain and form receptor complexes with RLKs to initiate PAMP-triggered immunity, e.g. RLCK AtBIK1 associates with the flagellin RLK AtFLS2 (Lu et al., 2010). Three RLCKs have so far been found to be activated by ROPs during plant defence. In A. thaliana, AtROP4 interacts with RLCK AtRBK1, which is locally upregulated upon P. infestans and Botrytis cinerea infection (Molendijk et al., 2008) and AtROP6 activates AtRLCKVI_A3 which controls resistance to the powdery mildew fungus Erysiphe cruciferarum (Reiner et al., 2015). In barley RLCK HVRBK1 is activated by ROP HvRACB upon infection with the powdery mildew fungus $B g h$ and influences microtubule organization (Huesmann et al., 2012). Like the RLCKs, MAPKs are also suggested to be activated by ROPs. For example rice OsMAPK6 is activated by ROP OsRAC1, which itself is activated upon PAMP/MAMP recognition (Lieberherr et al., 2005; Akamatsu et al., 2013; Nagano et al., 2016). In a similar way, Rho GTPases are involved in MAPK cascade activation in animals, suggesting that ROP-mediated MAPK signalling is a conserved mechanism in eukaryotes (Kawano et al., 2014). Apparently, RLKs can have a direct effect on the RCLK and MAMP pathways or act indirectly via ROPs. 


\section{Function of the exocyst complex in plant immunity}

One of the downstream signalling components of the above discussed ROP-pathway is the exocyst complex. The current knowledge on the role of exocyst subunits in plant immunity is summarized in Table 1. It is evident that from each exocyst subunit, one or multiple homologs are involved in plant defence, indicating that the exocyst complex as a whole is important for proper response against plant pathogens (Chapter 2; Du et al., 2018a). The exocyst complex is associated with immunity to multiple unrelated pathogen species and with PAMP-triggered immunity (Stegmann et al., 2012; Du et al., 2018a; Ma et al., 2018), highlighting its importance for basal plant defence. It is involved in cell wall reinforcement through callose deposition (Pecenkova et al., 2011; Du et al., 2018a; Kulich et al., 2018), which is emphasised by the local accumulation of exocyst subunits at infection sites (Chapter 4). Altogether, these findings indicate that the exocyst complex is involved in targeting defence-related exocytosis to infection sites. Besides their role as part of the exocyst complex, exocyst subunits might also function independent of the exocyst complex. One example is rice Exo70e1 which interacts with the protein Bph6 that confers resistance to plant hoppers and this exocyst subunits as such acts as a guardee of Bph6 (Guo et al., 2018a).

\section{Exocyst subunits accumulate at plant-microbe membrane interfaces}

The localization patterns of exocyst subunits during both pathogenic and beneficial plant-microbe interactions are highly similar (Figure 3; Chapter 4; Genre et al., 2012; Zhang et al., 2015; Gavrin et al., 2017). Exocyst subunits accumulate at hyphal penetration sites, regardless of whether the hyphae is from a pathogen or a beneficial arbuscular mycorrhizal (AM) fungus. Exocyst subunits accumulate predominantly at the tip of plant membranes surrounding intracellular, invasive and arbuscular hyphae (Figure 3; Chapter 4; Genre et al., 2012; Zhang et al., 2015; Gavrin et al., 2017). Similarly, one exocyst subunit was found to accumulate around the tips of plant-derived infection threads formed during Rhizobium symbiosis (Gavrin et al., 2017). Together, these localization patterns suggest that the same exocytotic pathway is recruited to the plant-microbe interface without a distinction between friends or foes. In the previous section I argued that polarized exocytosis during plant defence is likely orchestrated by the same signalling pathways that regulate polarized exocytosis during developmental processes (Figure 2). Here, I extend this idea and suggest that plant cells employ the same signalling pathways for polarization of secretion during tip growth, upon pathogen attack, and for regulating polarized exocytosis during interaction with beneficial microbes.

How are exocyst subunits recruited to infection sites? It is known from work on yeast that exocyst localization is mediated by the interaction with Rho GTPases and phosphatidylinositol-4,5-bisphosphate $\left(\mathrm{PIP}_{2}\right)$ in the membrane (Mei \& Guo, 2018). In A. thaliana the interaction of exocyst with ROPs is indirect via two exocyst subunits have been found to indirectly interact with ROPs via AtICR1 and AtRIC7 (Lavy et al., 2007; Hong et al., 2016). One exocyst subunit, namely AtSec3a, was found to interact with $\mathrm{PIP}_{2^{\prime}}$ however this interaction is not driving its localization to pollen tube tips (Bloch et 
al., 2016; Li, $\mathrm{Y}$ et al., 2017). Assuming that the pathways that target polarized secretion during defence and development are similar (Figure 2), then the ROP-mediated signalling pathway activated upon microbe recognition by RLKs might facilitate the recruitment of exocyst subunits to infection sites. ROPs themselves localize in distinct plasma membrane domains via posttranslational lipid modifications and the interaction with membrane lipids (Feiguelman et al., 2018). In a similar way, several RLKs that induce defence signalling, e.g. FLS2, form plasma membrane nanodomains (Bucherl et al., 2017; Ott, 2017). In plant cells, exocyst subunits localize at the plasma membrane in punctate structures, referred to as foci, which are reminiscent of lipid nanodomains (Box I and Chapter 4). Therefore, locally modifying the lipid composition of the plasma membrane and therefore these nanodomains, could be a possible way to recruit exocyst subunits to infection sites.

The question remains whether the observed recruitment of exocyst subunits to infection site is an advantage or disadvantage for pathogens. Pathogen infection is a two-step process consisting of a pre-invasive stage before a pathogen enters a plant cell and a post-invasive stage after invasion of the pathogen. Pre-invasive defence responses aim at preventing pathogen entry by for example reinforcement of the cell wall by callose deposition in papillae (Collinge, 2009). Since the exocyst complex is involved in callose deposition and papillae formation (Pecenkova et al., 2011; Du et al., 2018a; Kulich et al., 2018), it is likely that during the pre-invasive stage, exocyst subunit relocalization contributes to plant defence. In the post-invasive stage, it is more likely that exocyst subunit accumulation around intracellular growing hyphae is in favour of the pathogen, since these invasive hyphae need to be facilitated by the insertion of plant-derived membranes by exocytosis. However, we also observed deposition of autofluorescent material around intracellular growing hyphae (Chapter 4), which could be the result of exocyst-mediated exocytosis of defence-related materials and hence a disadvantage for the pathogen. So, this suggests that microbes can adjust the consequences of exocyst-mediated exocytosis in such a way that defence-related processes are reduced, while processes aiding microbial entry are enhanced. It remains to be studied how this is achieved, but effector proteins are probable candidates to be involved in the manipulation of exocyst-mediated exocytosis.

TABLE 1. The role of exocyst subunits in plant-microbe interactions. Summary based on studies in a variety of pathosystems and using different experimental approaches.

\begin{tabular}{|c|c|c|c|c|}
\hline $\begin{array}{l}\text { Exocyst } \\
\text { subunit }\end{array}$ & $\begin{array}{l}\text { Plant } \\
\text { species }^{\text {a }}\end{array}$ & Involved in / known to be ${ }^{\mathrm{b}}$ : & Methodc & Reference(s) \\
\hline Sec3 & $\mathrm{Nb}$ & callose deposition in response to pathogens & VIGS & Du et al., 2018a \\
\hline Sec3a & Os & PAMP-triggered immunity; resistance to Mo & CRISPR & Ma et al., 2018 \\
\hline Sec3a & $\mathrm{Pp}$ & recruited to Pc infection sites & Loc & Chapter 4 \\
\hline $\operatorname{Sec} 5$ & $\mathrm{Nb}$ & $\begin{array}{l}\text { PR1 secretion; callose deposition in response } \\
\text { to pathogens; defence against Bc, Pi, Pss }\end{array}$ & VIGS & $\begin{array}{l}\text { Du et al., 2015a; } \\
\text { Du et al., 2018a }\end{array}$ \\
\hline Sec5b and d & $\mathrm{Pp}$ & recruited to Pc infection sites & Loc & Chapter 4 \\
\hline Sec6 & $\mathrm{Nb}$ & $\begin{array}{l}\text { callose deposition in response to pathogens; } \\
\text { defence against } \mathrm{Bc}, \mathrm{Pi} \text {, Pss }\end{array}$ & VIGS & Du et al., 2018a \\
\hline
\end{tabular}


TABLE 1. (Continued)

\begin{tabular}{|c|c|c|c|c|}
\hline $\begin{array}{l}\text { Exocyst } \\
\text { subunit }\end{array}$ & $\begin{array}{l}\text { Plant } \\
\text { species }^{\text {a }}\end{array}$ & Involved in / known to be & Method ${ }^{\mathrm{C}}$ & Reference(s) \\
\hline Sec6 & $\mathrm{Pp}$ & recruited to Pc infection sites & Loc & Chapter 4 \\
\hline Sec8 & $\mathrm{Nb}$ & $\begin{array}{l}\text { callose deposition in response to pathogens; } \\
\text { defence against } \mathrm{Pi}\end{array}$ & VIGS & Du et al., 2018a \\
\hline Sec10 & $\mathrm{Nb}$ & $\begin{array}{l}\text { callose deposition in response to pathogens; } \\
\text { defence against } \mathrm{Bc} \text {, Pi, Pss }\end{array}$ & VIGS & Du et al., 2018a \\
\hline Sec15a & $\mathrm{Nb}$ & $\begin{array}{l}\text { callose deposition in response to pathogens; } \\
\text { defence against } \mathrm{Pi}\end{array}$ & VIGS & Du et al., 2018a \\
\hline Sec15b & $\mathrm{Nb}$ & callose deposition in response to pathogens & VIGS & Du et al., 2018a \\
\hline Exo70b & $\mathrm{Nb}$ & $\begin{array}{l}\text { callose deposition in response to pathogens; } \\
\text { defence against } \mathrm{Pi}\end{array}$ & VIGS & Du et al., 2018a \\
\hline Exo70b1 & At & $\begin{array}{l}\text { PAMP-triggered immunity; guarded by TIR- } \\
\text { NBS2; defence against Gc, Ha, Pst; recruited to } \\
\text { the plasma membrane by RIN4 }\end{array}$ & T-DNA & $\begin{array}{l}\text { Kulich et al., 2013; } \\
\text { Stegmann et al., 2013; } \\
\text { Zhao et al., 2015; Sabol } \\
\text { et al., } 2017\end{array}$ \\
\hline Exo70b2 & At & $\begin{array}{l}\text { PAMP-triggered immunity; papilla formation; } \\
\text { defence against } \mathrm{Bg}, \mathrm{Ha} \text {, Pst }\end{array}$ & T-DNA & $\begin{array}{l}\text { Pecenkova et al., } 2011 ; \\
\text { Stegmann et al., } 2012\end{array}$ \\
\hline Exo70c & $\mathrm{Nb}$ & callose deposition in response to pathogens & VIGS & Du et al., 2018a \\
\hline Exo70e & $\mathrm{Nb}$ & callose deposition in response to pathogens & VIGS & Du et al., 2018a \\
\hline Exo70e1 & Os & $\begin{array}{l}\text { guarded by resistance protein Bph6 against } \\
\text { planthoppers }\end{array}$ & OE; LoC & Guo et al., 2018a \\
\hline Exo70f & $\mathrm{Nb}$ & callose deposition in response to pathogens & VIGS & Du et al., $2018 a$ \\
\hline Exo70f & $\mathrm{Hv}$ & essential for host penetration resistance to $\mathrm{Bg}$ & TIGS & Ostertag et al., 2013 \\
\hline Exo70f 2 and $\mathrm{f} 3$ & Os & R-gene-mediated resistance against Mo & RNAi & Fujisaki et al., 2015 \\
\hline Exo70g & $\mathrm{Nb}$ & callose deposition in response to pathogens & VIGS & Du et al., 2018a \\
\hline Exo70h & $\mathrm{Nb}$ & callose deposition in response to pathogens & VIGS & Du et al., 2018a \\
\hline Exo70h1 & At & defence against Bg, Pst & T-DNA & Pecenkova et al., 2011 \\
\hline Exo70h4 & At & PMR4-mediated callose deposition & T-DNA & Kulich et al., 2018 \\
\hline Exo70i & Mt & $\begin{array}{l}\text { AM symbiosis; recruited to AM interface; } \\
\text { rhizobium symbiosis; recruited to infection } \\
\text { threads }\end{array}$ & TI; Loc & $\begin{array}{l}\text { Zhang et al., 2015; } \\
\text { Gavrin et al., } 2017\end{array}$ \\
\hline Exo84a & $\mathrm{Nb}$ & callose deposition in response to pathogens & VIGS & Du et al., 2018a \\
\hline Exo84b & $\mathrm{Nb}$ & defence against $\mathrm{Pi}$ & VIGS & Du et al., 2018 \\
\hline Exo84b & Mt & $\begin{array}{l}\text { formation of the arbuscular mycorrhizal } \\
\text { interface }\end{array}$ & Loc & Genre et al., 2012 \\
\hline
\end{tabular}

a Plants: At, Arabidopsis thaliana; Hv, Hordeum vulgare (Barley); Mt, Medicago truncatula; Nb Nicotiana benthamiana; Pp, Physcomitrella patens; Os, Oryza sativa (Rice).

b Pathogens: Bc, Botrytis cinerea; Bg, Blumeria graminis; Gc, Golovinomyces cichoracearum; Ha, Hyaloperonospora arabidopsidis; Mo, Magnaporthe oryzae; Pc, Phytophthora capsici; Pi, Phytophthora infestans; Pss, Pseudomonas syringae pv syringae; Pst, Pseudomonas syringae pv tomato.

'Methods: Loc: localization by microscopy; OE: Overexpression; RNAi: RNA-interference; T-DNA: T-DNA insertion mutant; TI: Transposon Insertion mutant; TIGS: Transient-Induced Gene Silencing; VIGS: Virus-Induced Gene Silencing. 


\section{Chapter 6}

Pathogenic interactions
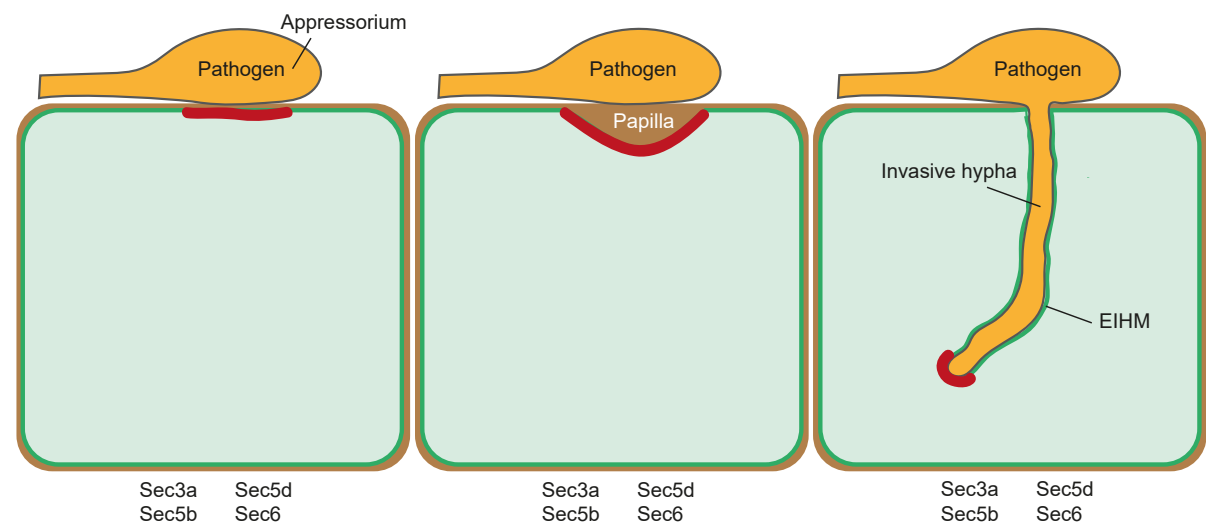

Beneficial interactions
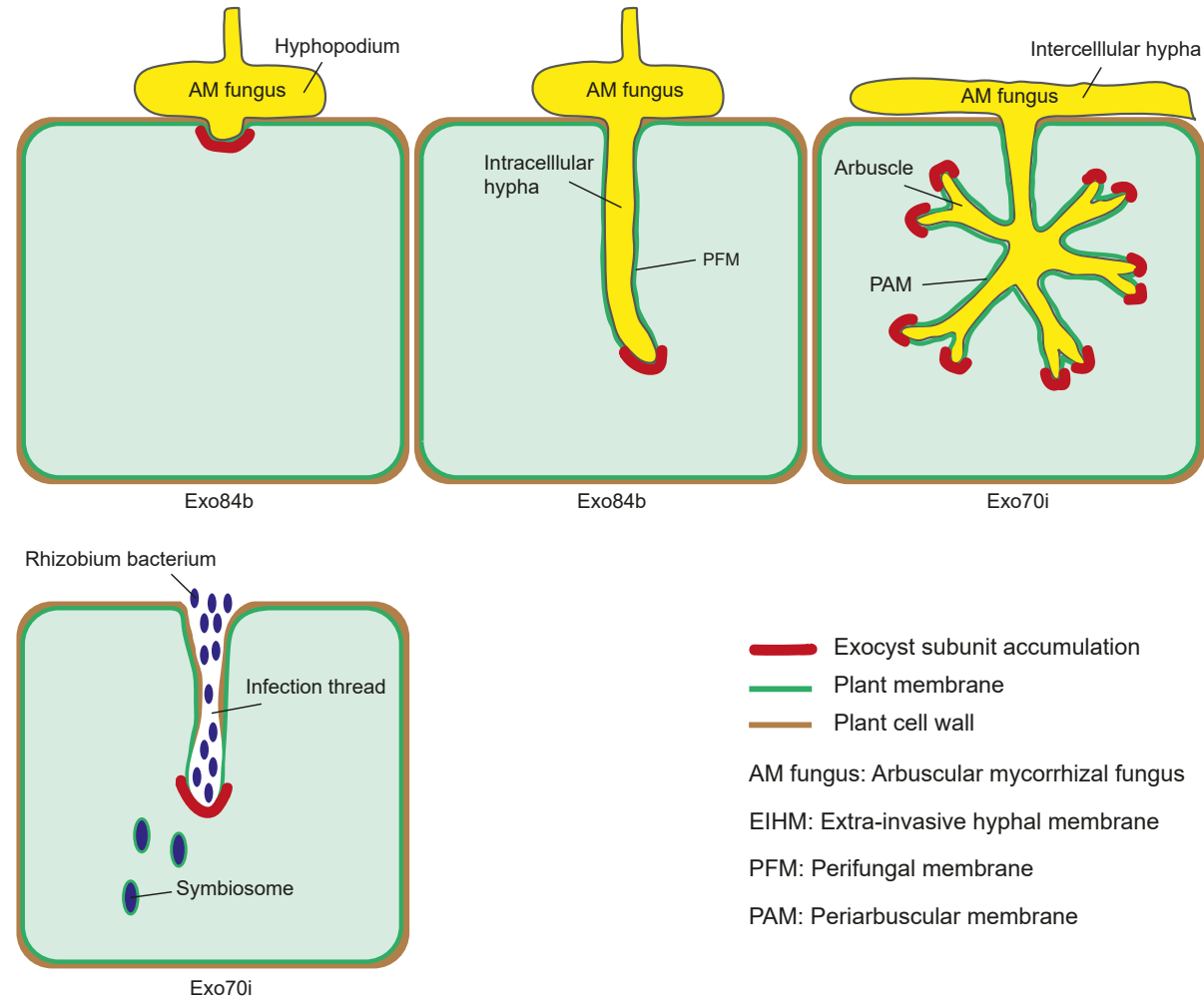

Exo84b

Exo70i

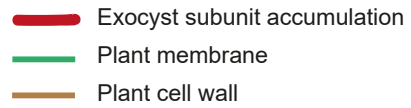

AM fungus: Arbuscular mycorrhizal fungus ElHM: Extra-invasive hyphal membrane PFM: Perifungal membrane

PAM: Periarbuscular membrane

FIGURE 3. Exocyst subunit localization patterns during pathogenic and beneficial plant-microbe interactions. Based on Chapter 4 and Genre et al., 2012, Zhang et al., 2015 and Gavrin et al., 2017. 


\section{Box I: The plant exocyst cabinet of curiosities}

The plant exocyst complex exhibits several noteworthy characteristics compared to the yeast and mammalian exocyst complex. Very obvious is the difference in gene copy number: in yeast and animals, each exocyst subunit is encoded by one single gene, while in plants the same subunit is often encoded by multiple paralogous genes, especially the Exo70 family that contains over 20 genes in Arabidopsis (Cvrckova et al., 2012). It is suggested that the plant specific expansion of the number of Exo70 genes is linked to functional specification, e.g. for different types of exocytosis (Zarsky et al., 2013; Martin-Urdiroz et al., 2016). Detailed analysis of tobacco Exo70 isoforms indeed shows that they localize to different regions of the pollen tube cell, and are linked to different cellular functions (Sekeres et al., 2017).

Other remarkable features of the plant exocyst complex are its subcellular localization and its dynamics. Microscopic analyses of several exocyst subunits revealed localization in punctate structures, called foci, decorating the plasma membrane of $A$. thaliana root, pollen, hypocotyl and P. patens protonema cells (Chapter 4; Hala et al., 2008; Fendrych et al., 2013; Zhang et al., 2013; Vukasinovic et al., 2017; van Gisbergen et al., 2018). These immobile foci exist at the plasma membrane for a certain time ranging from 4 to 12 seconds before disappearing again. In A. thaliana, the vesicle marker AtVAMP721 co-localizes with a small fraction of the AtExo84b foci, suggesting that the exocyst foci correspond with vesicle recruitment to the plasma membrane, and therefore with exocytotic events (Fendrych et al., 2013). The fluorescent brightness of these exocyst foci suggests that they consist of multiple exocyst subunit proteins and could represent hubs of exocytosis where multiple exocyst complexes accumulate.

Important questions regarding the assembly and disassembly of the plant exocyst complex still remain to be answered. In yeast, two subunits function as landmark proteins on the plasma membrane, i.e. Sec3 and Exo70, and the others arrive on vesicles (Mei \& Guo, 2018). However, in plants, none of the studied exocyst subunits appeared to be associated with exocytotic vesicles in the cytoplasm (Chapter 4; Fendrych et al., 2013; Zhang et al., 2013; Bloch et al., 2016). Although this suggests that the plant exocyst complex assembles differently form that of yeast, thorough co-localization analyses of exocyst subunits are required to form a more complete picture of exocyst assembly in plants.

With respect to $A$. thaliana Exo70e2, a unique localization pattern has been observed. It localizes in double membrane vesicle-like structures called Exocyst-Positive Organelles (EXPOs) and recruits other exocyst subunits to these EXPOs (Wang et al., 2010; Ding et al., 2014). EXPOs are distinct from autophagosomes and multi-vesicular bodies and have been implicated in stress-related unconventional exocytosis by fusing with the plasma membrane, thereby releasing a single membrane vesicle into the cell wall (Wang et al., 2010; Lin et al., 2015).

\section{Is the function of the exocyst complex in immunity conserved in other eukaryotes?}

What can we learn about the function of the exocyst complex in immunity from other eukaryotic organisms? In mammalian cells infected with the bacterium Salmonella typhimurium, several exocyst subunits are recruited to sites of invasion where they facilitate increased membrane expansion that will lead to internalization of Salmonella by 
macropinocytosis (Nichols \& Casanova, 2010). Likewise, in human cells infected with the parasite Trypasnosoma cruzi, two exocyst subunits accumulated at sites of infection and were required for plasma membrane repair by exocytosis that facilitated parasite invasion (Fernandes et al., 2015). This suggests that in a wider range of eukaryotes the exocyst complex is involved in facilitating entry of microbes through membrane expansion. It should be noted however that mammalian cells lack a cell wall and hence the role of the exocyst complex may differ compared to plants. The comparison with plants is limited by the absence of a cell wall in mammalian cells. Results from yeast cells, that do contain a cell wall, show that the exocyst complex is recruited from sites of polarized growth to wounding sites, thereby avoiding competition between cell polarization and wound healing (Kono et al., 2012). It could be that plant cells also employ this strategy.

\section{Pathogen effectors targeting the exocyst - a case study on the AVR1-Sec5 interaction}

As it stands, there is ample evidence that the plant exocyst plays a key role in plant immunity (Table 1) and this is supported by the fact that several pathogen effectors target exocyst subunits. Pathogens employ effector proteins to suppress host defence and to promote infection (Khan et al., 2018). To do so, many of these effectors target components of the host membrane trafficking machinery, including the exocyst complex (Chapter 1, Table 1). In Chapter 5, we focussed on the interaction between $P$. infestans effector AVR1 and exocyst subunit Sec5 of $P$. patens. In a previous study, AVR1 was found to interact with potato StSec5 and this was hypothesized to impair exocyst functioning in potato since it suppresses callose deposition (Du et al., 2015a). We first showed that AVR1 also interacts with $P$. patens Sec 5 homologs. We then generated transgenic $P$. patens lines with inducible expression of AVR1 and these lines showed similar developmental growth defects as Sec5silenced $P$. patens lines. This suggests that AVR1 also affects exocyst functioning in $P$. patens. However, the presence of AVR1 did not affect the localization of PpSec5 at the plasma membrane. Apparently, AVR1 impairs exocyst functioning without altering its subcellular localization. It could well be that AVR1 causes subtle changes in exocyst functioning, but as yet we have no tools to monitor this.

If indeed pathogens manipulate the plant exocyst complex to facilitate their entry, then it is conceivable that pathogen effectors play a role in this. AVR1 is an example of an effector that directly targets an exocyst subunit, but there are likely many more effectors that influence different aspects of exocyst-mediated exocytosis. The observed accumulation of exocyst subunits at tips of intracellular hypha of both a pathogen and a beneficial AM fungus (Figure 3), could be a result of manipulation of the exocyst complex by effectors. Similar to pathogenic microbes, AM fungi secrete effector proteins that participate in the establishment of symbiosis (Voß et al., 2018), and some of these effectors might target exocyst subunits. Pathogens like $P$. infestans secrete a large number of effector proteins (Haas et al., 2009) and their functions only start to be unravelled. Are there indeed different effectors that can distinguish between the pre-invasive and post-invasive stage, for example by differential expression, and thereby selectively influence exocyst-mediated 
secretion processes? Concerning the pre-invasive stage, how exactly do effectors impair exocyst complex function? Does for example the AVR1-Sec5 interaction affect the assembly of the exocyst complex and thereby its function? Could suppression of exocyst functioning result in a reduced tethering of vesicles at penetration attempt sites in the presence of AVR1?

Finally, why is it important to understand how pathogen effectors exactly suppress plant defence and in this case exocyst functioning? This knowledge could be instrumental in making precise changes and develop tailored resistances in crops. It would for example be interesting to improve plant resistance by preventing Sec5 from being targeted by AVR1. Therefore, it should first be determined where AVR1 binds to Sec5. Subsequently, Sec5 could be altered in such a way that AVR1 can no longer bind, while the function of Sec5 as exocyst subunit is not altered. Fundamental knowledge on the mode of action of pathogen effectors is essential to accomplish this.

\section{Conclusion and future perspectives}

In this thesis, we demonstrate the importance of a multidisciplinary approach combining phytopathology and cell biology to understand plant-pathogen interactions. We developed a novel model system to study cellular plant defence and combined this with the newest microscopy techniques to visualize protein localization during pathogenesis. We showed that the exocyst complex is involved in defence against a wide range of pathogens and were able to confirm the long anticipated localization of exocyst subunits at infection sites. To further tackle the biological significance of the recruitment of exocyst subunits to infection sites, it is recommended to study exocyst function during development in exocyst mutated or silenced $P$. patens lines. Are these lines showing an altered response to pathogens, i.e. more resistant or susceptible, and/or is the deposition of defence-related material affected? Is the tethering of exocytotic vesicles altered in these exocyst mutants? Does AVR1 affect tethering of exocytotic vesicles by targeting Sec5? The Physcomitrella-Phytophthora model is recommended to be used to study cellular defence responses, for example the involvement of ROP-related signalling pathways in orchestrating polarized exocytosis to infection sites. Do active $P$. patens ROPs accumulate at penetration attempt sites as hypothesized? Altogether, such studies in $P$. patens would further increase our understanding of general and pre-invasive defence responses and support the development of sustainable resistance in economically important crops to fulfil the rising demand for food. 


\section{Supplemental data}

SUPPLEMENTARY TABLE 1. ROP-signalling related proteins involved in tip growth and plant-microbe interactions.

\begin{tabular}{|c|c|c|c|c|}
\hline & System & Protein & $\begin{array}{l}\text { Plant } \\
\text { species }^{a}\end{array}$ & Reference(s) \\
\hline Signal & Pollen tubes & LURES & At & Takeuchi \& Higashiyama, 2016 \\
\hline \multirow[t]{11}{*}{ RLKs } & Pollen tubes & PRK1 & $\mathrm{SI}$ & Kaothien et al., 2005 \\
\hline & & PRK2 & SI & Kaothien et al., 2005 \\
\hline & & PRK2 & At & Chang et al., 2013 \\
\hline & & PRK6 & At & Takeuchi \& Higashiyama, 2016 \\
\hline & & FER & At & Duan et al., 2014 \\
\hline & Root hairs & FER & At & $\begin{array}{l}\text { Duan et al., 2010; Yu et al., 2012; } \\
\text { Huang., et al., } 2013\end{array}$ \\
\hline & Pathogen response & FER & At & $\begin{array}{l}\text { Kessler et al., 2010; Stegmann et al., } \\
2017\end{array}$ \\
\hline & & TEH1 & At & Qu et al., 2017 \\
\hline & & CEBiP/CERK1 & Os & Akamatsu et al., 2013 \\
\hline & Rhizobium symbiosis & NFP & Mt & Lei et al., 2015 \\
\hline & & NFRs & $\mathrm{Lj}$ & Ke et al., 2012 \\
\hline \multirow[t]{5}{*}{ ROPGEFS } & Pollen tubes & ROPGEF1,8,9,12,14 & At & $\begin{array}{l}\text { Zhang \& McCormick, 2007; Chang et } \\
\text { al., } 2013\end{array}$ \\
\hline & Root hairs & ROPGEF1,3,4 & At & $\begin{array}{l}\text { Duan et al., 2010; Denninger et al., } \\
2019\end{array}$ \\
\hline & Pathogen response & SWAP70 & Os & Yamaguchi et al., 2012 \\
\hline & & RACGEF1 & Os & Akamatsu et al., 2013 \\
\hline & & GEF4 & At & Qu et al., 2017 \\
\hline \multirow[t]{4}{*}{ ROPGAPs } & Pollen tubes & RhoGAP1 & $\mathrm{Nt}$ & Klahre \& Kost, 2006; Sun et al., 2015 \\
\hline & & REN1 & At & Hwang et al., 2008 \\
\hline & & ROPGAP1 & At & Fu et al., 2001; Hwang et al., 2010 \\
\hline & Pathogen response & MAPGAP & $\mathrm{Hv}$ & Hoefle et al., 2011 \\
\hline \multirow[t]{2}{*}{ GDIs } & Pollen tubes & RhoGDI2a, b & At & Hwang et al., 2010; Feng et al., 2016 \\
\hline & Root hairs + pollen tubes & RhoGDI1 & At & $\begin{array}{l}\text { Carol et al., 2005; Hwang et al., 2010; } \\
\text { Feng et al., } 2016\end{array}$ \\
\hline \multirow[t]{6}{*}{ ROPs } & Pollen tubes & RAC5 & $\mathrm{Nt}$ & $\begin{array}{l}\text { Kost et al., 1999; Klahre et al., 2006; } \\
\text { Klahre \& Kost, 2006; Sun et al., 2015 }\end{array}$ \\
\hline & & ROP1 & At & $\begin{array}{l}\text { Fu et al., 2001; Gu et al., 2005; Lee et } \\
\text { al., } 2008\end{array}$ \\
\hline & & ROP3 & At & Huang et al., 2014 \\
\hline & & ROP5 & At & Feng et al., 2016 \\
\hline & Root hairs & ROP1 & At & Venus \& Oelmuller, 2013 \\
\hline & & $\mathrm{ROP} 2$ & At & Jones et al., 2002 \\
\hline
\end{tabular}




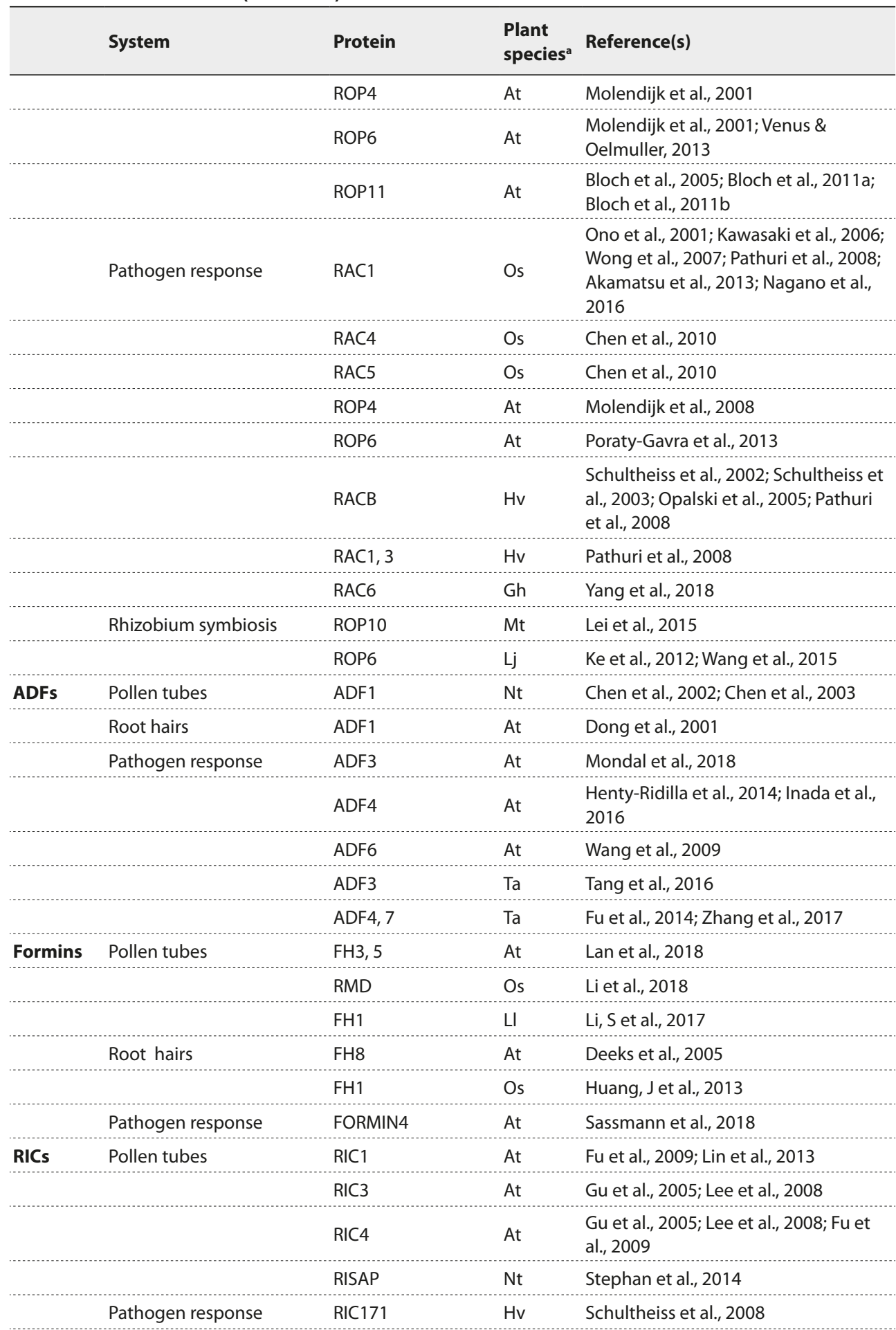


SUPPLEMENTARY TABLE 1. (Continued)

\begin{tabular}{|c|c|c|c|c|}
\hline & System & Protein & $\begin{array}{l}\text { Plant } \\
\text { species }^{a}\end{array}$ & Reference(s) \\
\hline ICRs & Pollen tubes & ICR1 & At & Li et al., 2008 \\
\hline \multirow[t]{2}{*}{ NADPH } & Root hairs & $\mathrm{RBOHC}$ & At & Carol et al., 2005 \\
\hline & Pathogen response & $\mathrm{RBOHB} / \mathrm{D}$ & Os & Nagano et al., 2016 \\
\hline \multirow[t]{3}{*}{ RLCKs } & Pathogen response & RBK1 & $\mathrm{Hv}$ & Huesmann et al., 2012 \\
\hline & & RBK1 & At & Molendijk et al., 2008 \\
\hline & & RLCKVI_A3 & At & Reiner et al., 2015 \\
\hline MAPK & Pathogen response & MAPK6 & Os & Lieberherr et al., 2005 \\
\hline \multirow[t]{7}{*}{ Exocyst } & Pollen tubes & $\operatorname{Sec} 3 a$ & At & Bloch et al., 2016; Li, Y et al., 2017 \\
\hline & & Sec5, 6, 15a & At & Hala et al., 2008 \\
\hline & & Sec8 & At & Cole et al., 2005; Hala et al., 2008 \\
\hline & & Exo70A1, B1 & $\mathrm{Nt}$ & Sekeres et al., 2017 \\
\hline & & Exo70C2 & $\mathrm{Nt}$ & Synek et al., 2017 \\
\hline & & Sec3 & $\mathrm{Zm}$ & Wen et al., 2005 \\
\hline & Root hairs & Exo70A1 & At & Synek et al., 2006 \\
\hline
\end{tabular}

a Plant species: At, Arabidopsis thaliana; Gh, Gossypium herbaceum (Cotton); Hv, Hordeum vulgare (Barley); Lj, Lillium japonicum; Mt, Medicago truncatula; Nt, Nicotiana tabacum (Tobacco); Os, Oryza sativa (Rice); SI, Solanum lycopersicum (Tomato); Ta, Triticum aestivum (Wheat); Zm, Zea mays (Maize). 
References 



\section{References}

Afzal AJ, da Cunha L, Mackey D. 2011. Separable fragments and membrane tethering of Arabidopsis RIN4 regulate its suppression of PAMP-triggered immunity. Plant Cell 23(10): 3798-3811.

Akamatsu A, Wong HL, Fujiwara M, et al. 2013. An OsCEBiP/OsCERK1-OsRacGEF1-OsRac1 module is an essential early component of chitin-induced rice immunity. Cell Host and Microbe 13(4): 465-476.

Akita M, Valkonen JPT. 2002. A novel gene family in moss (Physcomitrella patens) shows sequence homology and a phylogenetic relationship with the TIR-NBS class of plant disease resistance genes. Journal of Molecular Evolution 55(5): 595-605.

Akita M, Lehtonen MT, Koponen $\mathrm{H}$, et al. 2011. Infection of the Sunagoke moss panels with fungal pathogens hampers sustainable greening in urban environments. Science of the Total Environment 409(17): 3166-3173.

Akkerman M, Overdijk EJR, Schel JH, et al. 2011. Golgi body motility in the plant cell cortex correlates with actin cytoskeleton organization. Plant and Cell Physiology 52(10): 1844-1855.

Alberts B, Bray D, Hopkin K, et al. 2009. Essential Cell Biology. New York, USA: Garland Science.

Aldon D, Mbengue M, Mazars C, et al. 2018. Calcium signalling in plant biotic interactions. International Journal of Molecular Sciences 19(3): doi: 10.3390/ijms19030665.

An Q, Huckelhoven R, Kogel KH, et al. 2006. Multivesicular bodies participate in a cell wall-associated defence response in barley leaves attacked by the pathogenic powdery mildew fungus. Cellular Microbiology 8(6): 1009-1019.

Andersson RA, Akita M, Pirhonen M, et al. 2005. Moss-Erwinia pathosystem reveals possible similarities in pathogenesis and pathogen defense in vascular and nonvascular plants. Journal of General Plant Pathology 71(1): 23-28.

Ashton NW, Cove DJ. 1977. Isolation and preliminary characterization of auxotrophic and analog resistant mutants of moss, Physcomitrella patens. Molecular and General Genetics 154(1): 87-95.

Assaad FF, Qiu JL, Youngs H, et al. 2004. The PEN1 syntaxin defines a novel cellular compartment upon fungal attack and is required for the timely assembly of papillae. Molecular Biolology of the Cell 15(11): 5118-5129.

Aufrecht JA, Timm CM, Bible A, et al. 2018. Quantifying the spatiotemporal dynamics of plant root colonization by beneficial bacteria in a microfluidic habitat. Advanced Biosystems 2(6): doi: 10.1002/adbi.201800048.

Baluska F, Bacigalova K, Oud JL, et al. 1995. Rapid reorganization of microtubular cytoskeleton accompanies early changes in nuclear ploidy and chromatin structure in postmitotic cells of barley leaves infected with powdery mildew. Protoplasma 185(3-4): 140-151.

Bascom CS, Jr., Wu SZ, Nelson K, et al. 2016. Long-term growth of moss in microfluidic devices enables subcellular studies in development. Plant Physiol 172(1): 28-37.

Ben Khaled S, Postma J, Robatzek S. 2015. A moving view: subcellular trafficking processes in pattern recognition receptor-triggered plant immunity. Annual Review of Phytopathology 53: 379-402.

Berezin MY, Achilefu S. 2010. Fluorescence lifetime measurements and biological imaging. Chemical Reviews 110(5): 2641-2684.

Berken A, Wittinghofer A. 2008. Structure and function of Rho-type molecular switches in plants. Plant Physiology and Biochemistry 46(3): 380-393.

Berriri S, Garcia AV, Frei dit Frey N, et al. 2012. Constitutively active mitogen-activated protein kinase versions reveal functions of Arabidopsis MPK4 in pathogen defense signaling. Plant Cell 24(10): 4281-4293.

Bibeau JP, Kingsley JL, Furt F, et al. 2017. F-actin meditated focusing of vesicles at the cell tip is essential for polarized growth. Plant Physiology 176(1): 352-363.

Bloch D, Lavy M, Efrat Y, et al. 2005. Ectopic expression of an activated RAC in Arabidopsis disrupts membrane cycling. Molecular Biology of the Cell 16(4): 1913-1927.

Bloch D, Monshausen G, Gilroy S, et al. 2011a. Co-regulation of root hair tip growth by ROP GTPases and nitrogen source modulated pH fluctuations. Plant Signaling and Behavior 6(3): 426-429.

Bloch D, Monshausen G, Singer M, et al. 2011b. Nitrogen source interacts with ROP signalling in root hair tipgrowth. Plant Cell and Environment 34(1): 76-88.

Bloch D, Pleskot R, Pejchar P, et al. 2016. Exocyst SEC3 and phosphoinositides define sites of exocytosis in pollen tube initiation and growth. Plant Physiology 172(2): 980-1002.

Bogdanove AJ, Martin GB. 2000. AvrPto-dependent Pto-interacting proteins and AvrPto-interacting proteins in tomato. PNAS 97(16): 8836-8840. 
Bos JIB, Kanneganti TD, Young C, et al. 2006. The C-terminal half of Phytophthora infestans RXLR effector AVR3a is sufficient to trigger R3a-mediated hypersensitivity and suppress INF1-induced cell death in Nicotiana benthaminana. Plant Journal 48: 165-176.

Bos JIB, Armstrong MR, Gilroy EM, et al. 2010. Phytophthora infestans effector AVR3a is essential for virulence and manipulates plant immunity by stabilizing host E3 ligase CMPG1. PNAS 107: 9909-9914.

Boulter E, Garcia-Mata R. 2010. RhoGDI: A rheostat for the Rho switch. Small GTPases 1(1): 65-68.

Bouwmeester K, van Poppel PMJA, Govers F. 2009. Genome biology cracks enigmas of oomycete plant pathogens. In: Annual Plant Reviews 34, Molecular Aspects of Plant Disease Resistance (Parker J, ed). Wiley-Blackwell, United Kingdom, 102-134.

Bouwmeester K, de Sain M, Weide R, et al. 2011. The lectin receptor kinase LecRK-I.9 is a novel Phytophthora resistance component and a potential host target for a RXLR effector. PloS Pathogens 7(3): doi: 10.1371/ journal.ppat.1001327.

Boyd C, Hughes T, Pypaert M, et al. 2004. Vesicles carry most exocyst subunits to exocytic sites marked by the remaining two subunits, Sec3p and Exo70p. Journal of Cell Biology 167(5): 889-901.

Bozkurt TO, Belhaj K, Dagdas YF, et al. 2015. Rerouting of plant late endocytic trafficking toward a pathogen interface. Traffic 16(2): 204-226.

Bressendorff S. 2012. Immunity in the moss Physcomitrella patens. PhD thesis University of Copenhagen, DK.

Bressendorff S, Azevedo R, Kenchappa CS, et al. 2016. An innate immunity pathway in the moss Physcomitrella patens. Plant Cell 28(6): 1328-1342.

Brymora A, Valova VA, Larsen MR, et al. 2001. The brain exocyst complex interacts with RalA in a GTP-dependent manner: identification of a novel mammalian Sec3 gene and a second Sec15 gene. Journal of Biological Chemistry 276(32): 29792-29797.

Bucherl CA, Jarsch IK, Schudoma C, et al. 2017. Plant immune and growth receptors share common signalling components but localise to distinct plasma membrane nanodomains. eLife 6: doi: 10.7554/eLife.25114.

Cahill D, Rookes J, Michalczyk A, et al. 2002. Microtubule dynamics in compatible and incompatible interactions of soybean hypocotyl cells with Phytophthora sojae. Plant Pathology 51(5): 629-640.

Carella P, Gogleva A, Tomaselli M, et al. 2018. Phytophthora palmivora establishes tissue-specific intracellular infection structures in the earliest divergent land plant lineage. PNAS 115(16): doi: 10.1073/ pnas.1717900115.

Carella P, Schornack S. 2018. Manipulation of bryophyte hosts by pathogenic and symbiotic microbes. Plant and Cell Physiology 59(4): 651-660.

Carol RJ, Takeda S, Linstead P, et al. 2005. A RhoGDP dissociation inhibitor spatially regulates growth in root hair cells. Nature 438(7070): 1013-1016.

Caten CE, Jinks JL. 1967. Spontaneous variability of single isolates of Phytophthora infestans. Canadian Journal of Botany 46: 329-348.

Champouret N, Bouwmeester K, Rietman H, et al. 2009. Phytophthora infestans isolates lacking class I ipiO variants are virulent on Rpi-blb1 potato. Molecular Plant Microbe Interactions 22(12): 1535-1545.

Chang F, Gu Y, Ma H, et al. 2013. AtPRK2 promotes ROP1 activation via RopGEFs in the control of polarized pollen tube growth. Molecular Plant 6(4): 1187-1201.

Chaparro-Garcia A, Schwizer S, Sklenar J, et al. 2015. Phytophthora infestans RXLR-WY effector AVR3a associates with dynamin-related protein 2 required for endocytosis of the plant pattern recognition receptor FLS2. PLoS One 10(9): doi: 10.1371/journal.pone.0137071.

Chen CY, Wong El, Vidali L, et al. 2002. The regulation of actin organization by actin-depolymerizing factor in elongating pollen tubes. Plant Cell 14(9): 2175-2190.

Chen CY, Cheung AY, Wu HM. 2003. Actin-depolymerizing factor mediates Rac/Rop GTPase-regulated pollen tube growth. Plant Cell 15(1): 237-249.

Chen L, Shiotani K, Togashi T, et al. 2010. Analysis of the Rac/Rop small GTPase family in rice: expression, subcellular localization and role in disease resistance. Plant and Cell Physiology 51(4): 585-595.

Cheong MS, Kirik A, Kim JG, et al. 2014. AvrBsT acetylates Arabidopsis ACIP1, a protein that associates with microtubules and is required for immunity. PloS Pathogens 10(2): doi: 10.1371/journal.ppat.1003952.

Chong YT, Gidda SK, Sanford C, et al. 2010. Characterization of the Arabidopsis thaliana exocyst complex gene families by phylogenetic, expression profiling, and subcellular localization studies. New Phytologist 185(2): 401-419.

Cole RA, Synek L, Zarsky V, et al. 2005. SEC8, a subunit of the putative Arabidopsis exocyst complex, facilitates pollen germination and competitive pollen tube growth. Plant Physiology 138(4): 2005-2018. 
Cole RA, McInally SA, Fowler JE. 2014. Developmentally distinct activities of the exocyst enable rapid cell elongation and determine meristem size during primary root growth in Arabidopsis. BMC Plant Biology 14: doi: 10.1186/s12870-12014-10386-12870.

Collinge DB. 2009. Cell wall appositions: the first line of defence. Journal of Experimental Botany 60(2): $351-352$.

Collins NC, Thordal-Christensen H, Lipka V, et al. 2003. SNARE-protein-mediated disease resistance at the plant cell wall. Nature 425(6961): 973-977.

Cook DE, Mesarich CH, Thomma BP. 2015. Understanding plant immunity as a surveillance system to detect invasion. Annual Review of Phytopathology 53: 541-563.

Cove D. 2005. The moss Physcomitrella patens. Annual Review of Genetics 39: 339-358.

Cui F, Sun W, Kong X. 2018. RLCKs bridge plant immune receptors and MAPK cascades. Trends in Plant Science 23(12): 1039-1041.

Cvrckova F, Grunt M, Bezvoda R, et al. 2012. Evolution of the land plant exocyst complexes. Frontiers in Plant Science 3: doi: 10.3389/fpls.2012.00159.

Dagdas YF, Belhaj K, Maqbool A, et al. 2016. An effector of the Irish potato famine pathogen antagonizes a host autophagy cargo receptor. eLife 5: doi: 10.7554/eLife.10856.

Dagdas YF, Pandey P, Tumtas Y, et al. 2018. Host autophagy machinery is diverted to the pathogen interface to mediate focal defense responses against the Irish potato famine pathogen. eLife 7: doi: 10.7554/ eLife.37476.

Dao TT, Linthorst HJ, Verpoorte R. 2011. Chalcone synthase and its functions in plant resistance. Phytochemical Review 10(3): 397-412.

Davey ML, Currah RS. 2006. Interactions between mosses (Bryophyta) and fungi. Canadian Journal of Botany 84(10): 1509-1519.

Davey ML, Tsuneda A, Currah RS. 2009. Pathogenesis of bryophyte hosts by the ascomycete Atradidymella muscivora. Amarican Journal of Botany 96(7): 1274-1280.

Davey ML, Tsuneda A, Currah RS. 2010. Saprobic and parasitic interactions of Coniochaeta velutina with mosses. Botany-Botanique 88(3): 258-265.

Day B, Henty JL, Porter KJ, et al. 2011. The pathogen-actin connection: a platform for defense signaling in plants. Annual Review of Phytopathology 49: 483-506.

de Keijzer J, Kieft H, Ketelaar T, et al. 2017. Shortening of microtubule overlap regions defines membrane delivery sites during plant cytokinesis. Current Biology 27(4): 514-520.

Deeks MJ, Cvrckova F, Machesky LM, et al. 2005. Arabidopsis group le formins localize to specific cell membrane domains, interact with actin-binding proteins and cause defects in cell expansion upon aberrant expression. New Phytologist 168(3): 529-540.

Deeks MJ, Sanchez-Rodriguez C. 2016. Seeing is believing: cell biology at the plant-microbe interface. New Phytologist 211(1): 16-19.

Denninger P, Reichelt A, Schmidt VAF, et al. 2019. Distinct RopGEFs successively drive polarization and outgrowth of root hairs. Current Biology 29(11): 1854-1865.

Ding Y, Wang J, Lai JHC, et al. 2014. Exo70E2 is essential for exocyst subunit recruitment and EXPO formation in both plants and animals. Molecular Biology of the Cell 25(3): 412-426.

Dodds PN, Rathjen JP. 2010. Plant immunity: towards an integrated view of plant-pathogen interactions. Nature Review Genetics 11(8): 539-548.

Donahoo RS, Lamour KH. 2008. Interspecific hybridization and apomixis between Phytophthora capsici and Phytophthora tropicalis. Mycologia 100(6): 911-920.

Dong CH, Xia GX, Hong Y, et al. 2001. ADF proteins are involved in the control of flowering and regulate F-actin organization, cell expansion, and organ growth in Arabidopsis. Plant Cell 13(6): 1333-1346.

Drdova EJ, Synek L, Pecenkova T, et al. 2013. The exocyst complex contributes to PIN auxin efflux carrier recycling and polar auxin transport in Arabidopsis. Plant Journal 73(5): 709-719.

Driouich A, Jauneau A, Staehelin LA. 1997. 7-Dehydrobrefeldin A, a naturally occurring brefeldin A derivative, inhibits secretion and causes a cis-to-trans breakdown of Golgi stacks in plant cells. Plant Physiology 113(2): 487-492.

Du Y, Mpina MH, Birch PR, et al. 2015a. Phytophthora infestans RXLR effector AVR1 interacts with exocyst component Sec5 to manipulate plant immunity. Plant Physiology 169: 1975-1990.

Du Y, Berg JA, Govers F, Bouwmeester K. 2015b. Immune activation mediated by the late blight resistance protein R1 requires nuclear localization of R1 and the effector AVR1. New Phytologist 207: 735-747. 
Du Y, Overdijk EJR, Berg JA, et al. 2018a. Solanaceous exocyst subunits are involved in immunity to diverse plant pathogens. Journal of Experimental Botany 69(3): 655-666.

Du Y, Weide R, Zhao Z, Msimuko P, et al. 2018b. RXLR effector diversity in Phytophthora infestans isolates determines recognition by potato resistance proteins; the case study AVR1 and R1. Studies in Mycology 89: 85-93.

Duan Q, Kita D, Li C, et al. 2010. FERONIA receptor-like kinase regulates RHO GTPase signaling of root hair development. PNAS 107(41): 17821-17826.

Duan Q, Kita D, Johnson EA, et al. 2014. Reactive oxygen species mediate pollen tube rupture to release sperm for fertilization in Arabidopsis. Nature Communications 5: doi: 10.1038/ncomms4129.

Duman JG, Forte JG. 2003. What is the role of SNARE proteins in membrane fusion? American Journal of Physiology-Cell Physiology 285(2): C237-C249.

Eggert D, Naumann M, Reimer R, et al. 2014. Nanoscale glucan polymer network causes pathogen resistance. Science Reports 4: doi: 10.1038/srep04159.

El Oirdi M, Bouarab K. 2007. Plant signalling components EDS1 and SGT1 enhance disease caused by the necrotrophic pathogen Botrytis cinerea. New Phytologist 175(1): 131-139.

Ellinger D, Naumann M, Falter C, et al. 2013. Elevated early callose deposition results in complete penetration resistance to powdery mildew in Arabidopsis. Plant Physiology 161(3): 1433-1444.

Ellinger D, Voigt CA. 2014. Callose biosynthesis in Arabidopsis with a focus on pathogen response: what we have learned within the last decade. Annals of Botany 114(6): 1349-1358.

Erwin DC, Ribeiro OK. 1996. Phytophthora diseases worldwide. St. Paul, MN, US: American Phytopathological Society.

Etienne-Manneville S, Hall A. 2002. Rho GTPases in cell biology. Nature 420(6916): 629-635.

Faulkner C. 2015. A cellular backline: specialization of host membranes for defence. Journal of Experimental Botany 66(6): 1565-1571.

Feiguelman G, Fu Y, Yalovsky S. 2018. ROP GTPases structure-function and signaling pathways. Plant Physiology 176(1): 57-79.

Feijo JA, Moreno N. 2004. Imaging plant cells by two-photon excitation. Protoplasma 223(1): 1-32.

Fendrych M, Synek L, Pecenkova T, et al. 2010. The Arabidopsis exocyst complex is involved in cytokinesis and cell plate maturation. Plant Cell 22(9): 3053-3065.

Fendrych M, Synek L, Pecenkova T, et al. 2013. Visualization of the exocyst complex dynamics at the plasma membrane of Arabidopsis thaliana. Molecular Biology of the Cell 24(4): 510-520.

Feng QN, Kang H, Song SJ, et al. 2016. Arabidopsis RhoGDIs Are Critical for Cellular Homeostasis of Pollen Tubes. Plant Physiology 170(2): 841-856.

Fernandes MC, Corrotte M, Miguel DC, et al. 2015. The exocyst is required for trypanosome invasion and the repair of mechanical plasma membrane wounds. Journal of Cell Science 128(1): 27-32.

Forster H, Tyler BM, Coffey MD. 1994. Phytophthora sojae races have arisen by clonal evolution and by rare outcrosses. Molecular Plant-Microbe Interactions 7(6): 780-791.

Fry W. 2008. Phytophthora infestans: the plant (and R gene) destroyer. Molecular Plant Pathology 9(3): 385-402.

Fu Y, Wu G, Yang Z. 2001. Rop GTPase-dependent dynamics of tip-localized F-actin controls tip growth in pollen tubes. Journal of Cell Biology 152(5): 1019-1032.

Fu Y, Xu T, Zhu L, et al. 2009. A ROP GTPase signaling pathway controls cortical microtubule ordering and cell expansion in Arabidopsis. Current Biology 19(21): 1827-1832.

Fu Y, Duan X, Tang C, et al. 2014. TaADF7, an actin-depolymerizing factor, contributes to wheat resistance against Puccinia striiformis f. sp. tritici. Plant Journal 78(1): 16-30.

Fu Y. 2015. The cytoskeleton in the pollen tube. Current Opinion in Plant Biology 28: 111-119.

Fujisaki K, Abe Y, Ito A, et al. 2015. Rice Exo70 interacts with a fungal effector, AVR-Pii and is required for AVR-Piitriggered immunity. Plant Journal 88: 875-887.

Furt F, Liu YC, Bibeau JP, et al. 2013. Apical myosin XI anticipates F-actin during polarized growth of Physcomitrella patens cells. Plant Journal 73(3): 417-428.

Gabriels SH, Vossen JH, Ekengren SK, et al. 2007. An NB-LRR protein required for HR signalling mediated by both extra- and intracellular resistance proteins. Plant Journal 50(1): 14-28.

Gavrin A, Kulikova O, Bisseling T, et al. 2017. Interface symbiotic membrane formation in root nodules of Medicago truncatula: the role of synaptotagmins MtSyt1, MtSyt2 and MtSyt3. Frontiers in Plant Science 8: doi: 10.3389/ fpls.2017.00201.

Genre A, Ivanov S, Fendrych M, et al. 2012. Multiple exocytotic markers accumulate at the sites of perifungal membrane biogenesis in arbuscular mycorrhizas. Plant and Cell Physiology 53(1): 244-255. 
Goodin MM, Zaitlin D, Naidu RA, et al. 2008. Nicotiana benthamiana: its history and future as a model for plantpathogen interactions. Molecular Plant Microbe Interactions 21(8): 1015-1026.

Gooh K, Ueda M, Aruga K, et al. 2015. Live-cell imaging and optical manipulation of Arabidopsis early embryogenesis. Developmental Cell 34(2): 242-251.

Grebnev G, Ntefidou M, Kost B. 2017. Secretion and endocytosis in pollent tubes: models of tip growth in the spot light. Frontiers in Plant Science 8: doi: 10.3389/fpls.2017.00154.

Gross P, Julius C, Schmelzer E, et al. 1993. Translocation of cytoplasm and nucleus to fungal penetration sites is associated with depolymerization of microtubules and defense gene activation in infected, cultured parsley cells. EMBO Journal 12(5): 1735-1744.

Grossmann G, Krebs M, Maizel A, et al. 2018. Green light for quantitative live-cell imaging in plants. Journal of Cell Science 131(2): doi: 10.1242/jcs.209270.

Gu Y, Fu Y, Dowd P, et al. 2005. A Rho family GTPase controls actin dynamics and tip growth via two counteracting downstream pathways in pollen tubes. Journal of Cell Biology 169(1): 127-138.

Gu Y, Zavaliev R, Dong X. 2017. Membrane trafficking in plant immunity. Molecular Plant 10(8): 1026-1034.

Guo M, Kim P, Li G, et al. 2016. A bacterial effector co-opts calmodulin to target the plant microtubule network. Cell Host and Microbe 19(1): 67-78.

Guo J, Xu C, Wu D, et al. 2018a. Bph6 encodes an exocyst-localized protein and confers broad resistance to planthoppers in rice. Nature Genetics 50(2): 297-306.

Guo J, Xu C, Wu D, et al. 2018b. Bph6 encodes an exocyst-localized protein and confers broad resistance to planthoppers in rice. Nature Genetics: doi: 10.1038/s41588-41018-40039-41586.

Gyorgy B, Szabo TG, Pasztoi M, et al. 2011. Membrane vesicles, current state-of-the-art: emerging role of extracellular vesicles. Cellular and Molecular Life Sciences 68(16): 2667-2688.

Haas BJ, Kamoun S, Zody MC, et al. 2009. Genome sequence and analysis of the Irish potato famine pathogen Phytophthora infestans. Nature 461(7262): 393-398.

Hala M, Cole R, Synek L, et al. 2008. An exocyst complex functions in plant cell growth in Arabidopsis and tobacco. Plant Cell 20(5): 1330-1345.

Hardham AR. 2001. The cell biology behind Phytophthora pathogenicity. Australasian Plant Pathology 30(2): 9198.

Hardham AR. 2007. Cell biology of plant-oomycete interactions. Cell Microbiology 9(1): 31-39.

Hardham AR, Jones DA, Takemoto D. 2007. Cytoskeleton and cell wall function in penetration resistance. Current Opinion in Plant Biology 10(4): 342-348.

Hardham AR, Takemoto D, White RG. 2008. Rapid and dynamic subcellular reorganization following mechanical stimulation of Arabidopsis epidermal cells mimics responses to fungal and oomycete attack. BMC Plant Biology 8: doi: 10.1186/1471-2229-1188-1163.

Hardham AR. 2013. Microtubules and biotic interactions. Plant Journal 75(2): 278-289.

Heider MR, Munson M. 2012. Exorcising the exocyst complex. Traffic 13(7): 898-907.

Heider MR, Gu M, Duffy CM, et al. 2016. Subunit connectivity, assembly determinants and architecture of the yeast exocyst complex. Nature Structural and Molecular Biology 23(1): 59-66.

Henty-Ridilla JL, Shimono M, Li J, et al. 2013. The plant actin cytoskeleton responds to signals from microbeassociated molecular patterns. PloS Pathogens 9(4): doi: 10.1371/journal.ppat.1003290.

Henty-Ridilla JL, Li J, Day B, et al. 2014. Actin depolymerizing factor 4 regulates actin dynamics during innate immune signaling in Arabidopsis. Plant Cell 26(1): 340-352.

Hiwatashi Y, Obara M, Sato Y, et al. 2008. Kinesins are indispensable for interdigitation of phragmoplast microtubules in the moss Physcomitrella patens. Plant Cell 20(11): 3094-3106.

Hoefle C, Huesmann C, Schultheiss H, et al. 2011. A barley ROP GTPase ACTIVATING PROTEIN associates with microtubules and regulates entry of the barley powdery mildew fungus into leaf epidermal cells. Plant Cell 23(6): 2422-2439.

Hofte H. 2015. The yin and yang of cell wall integrity control: brassinosteroid and FERONIA signaling. Plant and Cell Physiology 56(2): 224-231.

Hong D, Jeon BW, Kim SY, et al. 2016. The ROP2-RIC7 pathway negatively regulates light-induced stomatal opening by inhibiting exocyst subunit Exo70B1 in Arabidopsis. New Phytologist 209(2): 624-635.

Hsu SC, Ting AE, Hazuka CD, et al. 1996. The mammalian brain rsec6/8 complex. Neuron 17(6): 1209-1219.

Huang J, Gu M, Lai Z, et al. 2010. Functional analysis of the Arabidopsis PAL gene family in plant growth, development, and response to environmental stress. Plant Physiology 153(4): 1526-1538.

Huang GQ, Li E, Ge FR, et al. 2013. Arabidopsis RopGEF4 and RopGEF10 are important for FERONIA-mediated developmental but not environmental regulation of root hair growth. New Phytologist 200(4): 1089-1101. 
Huang J, Kim CM, Xuan YH, et al. 2013. Formin homology 1 (OsFH1) regulates root-hair elongation in rice (Oryza sativa). Planta 237(5): 1227-1239.

Huang JB, Liu H, Chen M, et al. 2014. ROP3 GTPase contributes to polar auxin transport and auxin responses and is important for embryogenesis and seedling growth in Arabidopsis. Plant Cell 26(9): 3501-3518.

Huckelhoven R, Panstruga R. 2011. Cell biology of the plant-powdery mildew interaction. Current Opinion in Plant Biology 14(6): 738-746.

Huesmann C, Reiner T, Hoefle C, et al. 2012. Barley ROP binding kinase1 is involved in microtubule organization and in basal penetration resistance to the barley powdery mildew fungus. Plant Physiology 159(1): 311-320.

Humbert C, Aimé S, Alabouvette C, et al. 2015. Remodelling of actin cytoskeleton in tomato cells in response to inoculation with a biocontrol strain of Fusarium oxysporum in comparison to a pathogenic strain. Plant Pathology 64(6): 1366-1374.

Hussey PJ, Ketelaar T, Deeks MJ. 2006. Control of the actin cytoskeleton in plant cell growth. Annual Reviews Plant Biology 57: 109-125.

Hwang JU, Vernoud V, Szumlanski A, et al. 2008. A tip-localized RhoGAP controls cell polarity by globally inhibiting Rho GTPase at the cell apex. Current Biology 18(24): 1907-1916.

Hwang JU, Wu G, Yan A, et al. 2010. Pollen-tube tip growth requires a balance of lateral propagation and global inhibition of Rho-family GTPase activity. Journal of Cell Science 123(3): 340-350.

Inada N, Ueda T. 2014. Membrane trafficking pathways and their roles in plant-microbe interactions. Plant and Cell Physiology 55(4): 672-686.

Inada N, Higaki T, Hasezawa S. 2016. Nuclear function of subclass I actin-depolymerizing factor contributes to susceptibility in Arabidopsis to an adapted powdery mildew fungus. Plant Physiology 170(3): 1420-1434.

Ivanov S, Fedorova EE, Limpens E, et al. 2012. Rhizobium-legume symbiosis shares an exocytotic pathway required for arbuscule formation. PNAS 109(21): 8316-8321.

James P, HalladayJ, Craig EA. 1996. Genomic libraries and a host strain designed for highly efficient two-hybrid selection in yeast. Genetics 144: 1425-1436.

Jahn R, Scheller RH. 2006. SNAREs-engines for membrane fusion. Nature Reviews Molecular Cell Biology 7(9): 631643.

Jarosch B, Collins NC, Zellerhoff N, et al. 2005. RAR1, ROR1, and the actin cytoskeleton contribute to basal resistance to Magnaporthe grisea in barley. Molecular Plant Microbe Interactions 18(5): 397-404.

Jiang RHY, Tripathy S, Govers F, et al. 2008. RXLR effector reservoir in two Phytophthora species is dominated by a single rapidly evolving superfamily with more than 700 members. PNAS 105: 4874-4879.

Jiang H, Xu Z, Aluru MR, et al. 2014. Plant chip for high-throughput phenotyping of Arabidopsis. Lab Chip 14(7): 1281-1293.

Jones MA, Shen JJ, Fu Y, et al. 2002. The Arabidopsis Rop2 GTPase is a positive regulator of both root hair initiation and tip growth. Plant Cell 14(4): 763-776.

Jones JD, Dangl JL. 2006. The plant immune system. Nature 444(7117): 323-329.

Judelson HS, Blanco FA. 2005. The spores of Phytophthora: weapons of the plant destroyer. Nature Review Microbiology 3(1): 47-58.

Kamoun S, Furzer O, Jones JD, et al. 2015. The Top 10 oomycete pathogens in molecular plant pathology. Molecular Plant Pathology 16(4): 413-434.

Kang Y, Jelenska J, Cecchini NM, et al. 2014. HopW1 from Pseudomonas syringae disrupts the actin cytoskeleton to promote virulence in Arabidopsis. PloS Pathogens 10(6): doi: 10.1371/journal.ppat.1004232.

Kankanala P, Czymmek K, Valent B. 2007. Roles for rice membrane dynamics and plasmodesmata during biotrophic invasion by the blast fungus. Plant Cell 19(2): 706-724.

Kaothien P, Ok SH, Shuai B, et al. 2005. Kinase partner protein interacts with the LePRK1 and LePRK2 receptor kinases and plays a role in polarized pollen tube growth. Plant Journal 42(4): 492-503.

Kawano Y, Kaneko-Kawano T, Shimamoto K. 2014. Rho family GTPase-dependent immunity in plants and animals. Frontiers in Plant Science 5: doi: 10.3389/fpls.2014.00522.

Kawasaki T, Koita H, Nakatsubo T, et al. 2006. Cinnamoyl-CoA reductase, a key enzyme in lignin biosynthesis, is an effector of small GTPase Rac in defense signaling in rice. PNAS 103(1): 230-235.

Ke D, Fang Q, Chen C, et al. 2012. The small GTPase ROP6 interacts with NFR5 and is involved in nodule formation in Lotus japonicus. Plant Physiology 159(1): 131-143.

Kee Y, Yoo JS, Hazuka CD, et al. 1997. Subunit structure of the mammalian exocyst complex. PNAS 94(26): 1443814443.

Kessler SA, Shimosato-Asano H, Keinath NF, et al. 2010. Conserved molecular components for pollen tube reception and fungal invasion. Science 330(6006): 968-971. 
Ketelaar T. 2013. The actin cytoskeleton in root hairs: all is fine at the tip. Current Opinion in Plant Biology 16(6): 749-756.

Khan M, Seto D, Subramaniam R, et al. 2018. Oh, the places they'll go! A survey of phytopathogen effectors and their host targets. Plant Journal 93(4): 651-663.

Kim H, O'Connell R, Maekawa-Yoshikawa M, et al. 2014. The powdery mildew resistance protein RPW8.2 is carried on VAMP721/722 vesicles to the extrahaustorial membrane of haustorial complexes. Plant Journal 79(5): 835-847.

King SR, McLellan H, Boevink PC, et al. 2014. Phytophthora infestans RXLR effector PexRD2 interacts with host MAPKKKE to suppress plant immune signaling. Plant Cell 26: 1345-1359.

Klahre U, Becker C, Schmitt AC, et al. 2006. Nt-RhoGDI2 regulates Rac/Rop signaling and polar cell growth in tobacco pollen tubes. Plant Journal 46(6): 1018-1031.

Klahre U, Kost B. 2006. Tobacco RhoGTPase ACTIVATING PROTEIN1 spatially restricts signaling of RAC/Rop to the apex of pollen tubes. Plant Cell 18(11): 3033-3046.

Knight C, Perroud P-F, Cove D. 2009. The moss Physcomitrella. West Sussex, UK: Blackwell Publishing.

Kobayashi Y, Yamada M, Kobayashi I, et al. 1997. Actin microfilaments are required for the expression of nonhost resistance in higher plants. Plant and Cell Physiology 38(6): 725-733.

Kobayashi I, Hakuno H. 2003. Actin-related defense mechanism to reject penetration attempt by a non-pathogen is maintained in tobacco BY-2 cells. Planta 217(2): 340-345.

Kodama Y. 2016. Time gating of chloroplast autofluorescence allows clearer fluorescence imaging in planta. PLoS One 11(3): doi: 10.1371/journal.pone.0152484.

Koh S, Andre A, Edwards $\mathrm{H}$, et al. 2005. Arabidopsis thaliana subcellular responses to compatible Erysiphe cichoracearum infections. Plant Journal 44(3): 516-529.

Kono K, Saeki Y, Yoshida S, et al. 2012. Proteasomal degradation resolves competition between cell polarization and cellular wound healing. Cell 150(1): 151-164.

Kost B, Lemichez E, Spielhofer P, et al. 1999. Rac homologues and compartmentalized phosphatidylinositol 4, 5-bisphosphate act in a common pathway to regulate polar pollen tube growth. Journal of Cell Biology 145(2): 317-330.

Kroon LP, Brouwer H, de Cock AW, et al. 2012. The genus Phytophthora anno 2012. Phytopathology 102(4): 348364.

Kubo M, Imai A, Nishiyama T, et al. 2013. System for stable beta-estradiol-inducible gene expression in the moss Physcomitrella patens. PLoS One 8: e77356.

Kulich I, Pecenkova T, Sekeres J, et al. 2013. Arabidopsis exocyst subcomplex containing subunit EXO70B1 is involved in autophagy-related transport to the vacuole. Traffic 14(11): 1155-1165.

Kulich I, Zarsky V. 2014. Autophagy-related direct membrane import from ER/cytoplasm into the vacuole or apoplast: a hidden gateway also for secondary metabolites and phytohormones? International Journal of Molecular Sciences 15(5): 7462-7474.

Kulich I, Vojtikova Z, Glanc M, et al. 2015. Cell wall maturation of Arabidopsis trichomes is dependent on exocyst subunit EXO70H4 and involves callose deposition. Plant Physiology 168(1): 120-131.

Kulich I, Vojtikova Z, Sabol P, et al. 2018. Exocyst subunit EXO70H4 has a specific role in callose synthase secretion and silica accumulation. Plant Physiology 176(3): 2040-2051.

Kwon C, Neu C, Pajonk S, et al. 2008. Co-option of a default secretory pathway for plant immune responses. Nature 451(7180): 835-840.

Lamour KH, Stam R, Jupe J, et al. 2012. The oomycete broad-host-range pathogen Phytophthora capsici. Molecular Plant Pathology 13(4): 329-337.

Lan Y, Liu X, Fu Y, et al. 2018. Arabidopsis class I formins control membrane-originated actin polymerization at pollen tube tips. PLoS Genetics 14(11): doi: 10.1371/journal.pgen.1007789.

Lavy M, Bloch D, Hazak O, et al. 2007. A Novel ROP/RAC effector links cell polarity, root-meristem maintenance, and vesicle trafficking. Current Biology 17(11): 947-952.

Le Bail A, Scholz S, Kost B. 2013. Evaluation of reference genes for RT qPCR analyses of structure-specific and hormone regulated gene expression in Physcomitrella patens gametophytes. PLoS One 8(8): doi: 10.1371/ journal.pone.0070998.

Leary AY, Sanguankiattichai N, Duggan C, et al. 2018. Modulation of plant autophagy during pathogen attack. Journal of Experimental Botany 69(6): 1325-1333.

Leborgne-Castel N, Bouhidel K. 2014. Plasma membrane protein trafficking in plant-microbe interactions: a plant cell point of view. Frontiers in Plant Science 5: doi: 10.3389/fpls.2014.00735. 
Lee YJ, Szumlanski A, Nielsen E, et al. 2008. Rho-GTPase-dependent filamentous actin dynamics coordinate vesicle targeting and exocytosis during tip growth. Journal of Cell Biology 181(7): 1155-1168.

Lee AH, Hurley B, Felsensteiner C, et al. 2012. A bacterial acetyltransferase destroys plant microtubule networks and blocks secretion. PloS Pathogens 8(2): doi: 10.1371/journal.ppat.1002523.

Lehtonen MT, Akita M, Kalkkinen N, et al. 2009. Quickly-released peroxidase of moss in defense against fungal invaders. New Phytologist 183(2): 432-443.

Lehtonen MT, Akita M, Frank W, et al. 2012a. Involvement of a class III peroxidase and the mitochondrial protein TSPO in oxidative burst upon treatment of moss plants with a fungal elicitor. Molecular Plant Microbe Interactions 25(3): 363-371.

Lehtonen MT, Marttinen EM, Akita M, et al. 2012b. Fungi infecting cultivated moss can also cause diseases in crop plants. Annals of Applied Biology 160(3): 298-307.

Lehtonen MT, Takikawa Y, Ronnholm G, et al. 2014. Protein secretome of moss plants (Physcomitrella patens) with emphasis on changes induced by a fungal elicitor. Journal of Proteome Research 13(2): 447-459.

Lei MJ, Wang Q, Li X, et al. 2015. The small GTPase ROP10 of Medicago truncatula is required for both tip growth of root hairs and nod factor-induced root hair deformation. Plant Cell 27(3): 806-822.

Li S, Gu Y, Yan A, et al. 2008. RIP1 (ROP Interactive Partner 1)/ICR1 marks pollen germination sites and may act in the ROP1 pathway in the control of polarized pollen growth. Molecular Plant 1(6): 1021-1035.

Li HL, Wang HY, Hao XY, et al. 2010. Effects of microtubule polymerization inhibitor on the hypersensitive response of wheat induced by the non-host pathogen Sphaerotheca fuliginea. Agricultural Sciences in China 9(3): 378-382.

Li SP, Chen M, Yu DL, et al. 2013. EXO70A1-mediated vesicle trafficking is critical for tracheary element development in Arabidopsis. Plant Cell 25(5): 1774-1786.

Li J, Henty-Ridilla JL, Staiger BH, et al. 2015. Capping protein integrates multiple MAMP signalling pathways to modulate actin dynamics during plant innate immunity. Nature Commun 6: doi: 10.1038/ncomms8206.

Li S, Dong H, Pei W, et al. 2017. LIFH1-mediated interaction between actin fringe and exocytic vesicles is involved in pollen tube tip growth. New Phytologist 214(2): 745-761.

Li Y, Tan X, Wang M, et al. 2017. Exocyst subunit SEC3A marks the germination site and is essential for pollen germination in Arabidopsis thaliana. Science Reports 7: doi: 10.1038/srep40279.

Li G, Yang X, Zhang X, et al. 2018. Rice morphology determinant-mediated actin filament organization contributes to pollen tube growth. Plant Physiology 177(1): 255-270.

Li P, Day B. 2019. Battlefield cytoskeleton: turning the tide on plant immunity. Molecular Plant Microbe Interactions 32(1): 25-34.

Lieberherr D, Thao NP, Nakashima A, et al. 2005. A sphingolipid elicitor-inducible mitogen-activated protein kinase is regulated by the small GTPase OsRac1 and heterotrimeric G-protein in rice Plant Physiology 138(3): 1644-1652.

Lin D, Cao L, Zhou Z, et al. 2013. Rho GTPase signaling activates microtubule severing to promote microtubule ordering in Arabidopsis. Current Biology 23(4): 290-297.

Lin Y, Ding Y, Wang J, et al. 2015. Exocyst-positive organelles and autophagosomes are distinct organelles in plants. Plant Physiology 169(3): 1917-1932.

Lopez-Obando M, Hoffmann B, Gery C, et al. 2016. Simple and efficient targeting of multiple genes through CRISPR-Cas9 in Physcomitrella patens. G3 6(11): 3647-3653.

Lu D, Wu S, Gao X, et al. 2010. A receptor-like cytoplasmic kinase, BIK1, associates with a flagellin receptor complex to initiate plant innate immunity. PNAS 107(1): 496-501.

Lu YJ, Schornack S, Spallek T, et al. 2012. Patterns of plant subcellular responses to successful oomycete infections reveal differences in host cell reprogramming and endocytic trafficking. Cellular Microbiology 14(5): 682697.

Ma J, Chen J, Wang M, et al. 2018. Disruption of OsSEC3A increases the content of salicylic acid and induces plant defense responses in rice. Journal of Experimental Botany 69(5): 1051-1064.

Maclver SK, Hussey PJ. 2002. The ADF/cofilin family: actin-remodeling proteins. Genome Biology 3(5): reviews3007.

Martin-Urdiroz M, Deeks MJ, Horton CG, et al. 2016. The exocyst complex in health and disease. Frontiers in Cell and Developmental Biology 4: doi: 10.3389/fcell.2016.00024.

Martinez-Abaigar J, Nunez-Olivera E, Matcham HW, et al. 2005. Interactions between parasitic fungi and mosses: pegged and swollen-tipped rhizoids in Funaria and Bryum. Journal of Bryology 27: 47-53.

Massalha H, Korenblum E, Malitsky S, et al. 2017. Live imaging of root-bacteria interactions in a microfluidics setup. PNAS 114(17): 4549-4554. 
Matern HT, Yeaman C, Nelson WJ, et al. 2001. The Sec6/8 complex in mammalian cells: characterization of mammalian Sec3, subunit interactions, and expression of subunits in polarized cells. PNAS 98(17): 96489653.

Matheson LA, Hanton SL, Brandizzi F. 2006. Traffic between the plant endoplasmic reticulum and Golgi apparatus: to the Golgi and beyond. Current Opinion in Plant Biology 9(6): 601-609.

Mayers JR, Hu T, Wang C, et al. 2017. SCD1 and SCD2 form a complex that functions with the exocyst and RabE1 in exocytosis and cytokinesis. Plant Cell 29: 2610-2625.

McElroy D, Zhang W, Cao J, et al. 1990. Isolation of an efficient actin promoter for use in rice transformation. Plant Cell 2(2): 163-171.

McLellan H, Boevink PC, Armstrong MR, et al. 2013. An RxLR effector from Phytophthora infestans prevents relocalisation of two plant NAC transcription factors from the endoplasmic reticulum to the nucleus. PLoS Pathogens 9: e1003670.

Mei K, Guo W. 2018. The exocyst complex. Current Biology 28(17): R922-R925.

Meijer HJ, Hua C, Kots K, et al. 2014. Actin dynamics in Phytophthora infestans; rapidly reorganizing cables and immobile, long-lived plaques. Cellular Microbiology 16(6): 948-961.

Menand B, Calder G, Dolan L. 2007. Both chloronemal and caulonemal cells expand by tip growth in the moss Physcomitrella patens. Journal of Experimental Botany 58(7): 1843-1849.

Meyer D, Pajonk S, Micali C, et al. 2009. Extracellular transport and integration of plant secretory proteins into pathogen-induced cell wall compartments. Plant Journal 57(6): 986-999.

Micali CO, Neumann U, Grunewald D, et al. 2011. Biogenesis of a specialized plant-fungal interface during host cell internalization of Golovinomyces orontii haustoria. Cellular Microbioly 13(2): 210-226.

Miklis M, Consonni C, Bhat RA, et al. 2007. Barley MLO modulates actin-dependent and actin-independent antifungal defense pathways at the cell periphery. Plant Physiology 144(2): 1132-1143.

Mochizuki S, Minami E, Nishizawa Y. 2015. Live-cell imaging of rice cytological changes reveals the importance of host vacuole maintenance for biotrophic invasion by blast fungus, Magnaporthe oryzae. Microbiology Open 4(6): 952-966.

Molendijk AJ, Bischoff F, Rajendrakumar CS, et al. 2001. Arabidopsis thaliana Rop GTPases are localized to tips of root hairs and control polar growth. EMBO Journal 20(11): 2779-2788.

Molendijk AJ, Ruperti B, Singh MK, et al. 2008. A cysteine-rich receptor-like kinase NCRK and a pathogen-induced protein kinase RBK1 are Rop GTPase interactors. Plant Journal 53(6): 909-923.

Mondal HA, Louis J, Archer L, et al. 2018. Arabidopsis ACTIN-DEPOLYMERIZING FACTOR3 is required for controlling aphid feeding from the phloem. Plant Physiology 176(1): 879-890.

Mukhtar MS, Carvunis AR, Dreze M, et al. 2011. Independently evolved virulence effectors converge onto hubs in a plant immune system network. Science 333(6042): 596-601.

Munson M, Novick P. 2006. The exocyst defrocked, a framework of rods revealed. Nature Structural and Molecular Biology 13(7): 577-581.

Nakaoka Y, Miki T, Fujioka R, et al. 2012. An inducible RNA interference system in Physcomitrella patens reveals a dominant role of augmin in phragmoplast microtubule generation. Plant Cell 24: 1478-1493.

Nagano M, Ishikawa T, Fujiwara M, et al. 2016. Plasma membrane microdomains are essential for Rac1-RbohB/Hmediated immunity in rice. Plant Cell 28(8): 1966-1983.

Nichols CD, Casanova JE. 2010. Salmonella-directed recruitment of new membrane to invasion foci via the host exocyst complex. Current Biology 20(14): 1316-1320.

Nielsen ME, Feechan A, Bohlenius H, et al. 2012. Arabidopsis ARF-GTP exchange factor, GNOM, mediates transport required for innate immunity and focal accumulation of syntaxin PEN1. PNAS 109(28): 11443-11448.

Nishiyama T, Hiwatashi Y, Sakakibara I, et al. 2000. Tagged mutagenesis and gene-trap in the moss, Physcomitrella patens by shuttle mutagenesis. DNA Research 7(1): 9-17.

Nomura K, Debroy S, Lee YH, et al. 2006. A bacterial virulence protein suppresses host innate immunity to cause plant disease. Science 313(5784): 220-223.

Nomura K, Mecey C, Lee YN, et al. 2011. Effector-triggered immunity blocks pathogen degradation of an immunity-associated vesicle traffic regulator in Arabidopsis. PNAS 108(26): 10774-10779.

Oda Y, lida Y, Nagashima Y, et al. 2015. Novel coiled-coil proteins regulate exocyst association with cortical microtubules in xylem cells via the conserved oligomeric golgi-complex 2 protein. Plant and Cell Physiology 56(2): 277-286.

Oliver JP, Castro A, Gaggero C, et al. 2009. Pythium infection activates conserved plant defense responses in mosses. Planta 230(3): 569-579. 
Ono E, Wong HL, Kawasaki T, et al. 2001. Essential role of the small GTPase Rac in disease resistance of rice. PNAS 98(2): 759-764.

Opalski KS, Schultheiss H, Kogel KH, et al. 2005. The receptor-like MLO protein and the RAC/ROP family G-protein RACB modulate actin reorganization in barley attacked by the biotrophic powdery mildew fungus Blumeria graminis f.sp hordei. Plant Journal 41(2): 291-303.

Ostertag M, Stammler J, Douchkov D, et al. 2013. The conserved oligomeric Golgi complex is involved in penetration resistance of barley to the barley powdery mildew fungus. Molecular Plant Pathology 14(3): 230-240.

Ott T. 2017. Membrane nanodomains and microdomains in plant-microbe interactions. Current Opinion in Plant Biology 40: 82-88.

Overdijk EJR, De Keijzer J, De Groot D, et al. 2016. Interaction between the moss Physcomitrella patens and Phytophthora: a novel pathosystem for live-cell imaging of subcellular defence. Journal of Microscopy 263(2): 171-180.

Pan X, Chen J, Yang Z. 2015. Auxin regulation of cell polarity in plants. Current Opinion in Plant Biology 28: $144-153$.

Parashar A, Pandey S. 2011. Plant-in-chip: Microfluidic system for studying root growth and pathogenic interactions in Arabidopsis. Applied Physics Letters 98(26): doi: 10.1063/1061.3604788.

Paredez AR, Somerville CR, Ehrhardt DW. 2006. Visualization of cellulose synthase demonstrates functional association with microtubules. Science 312(5779): 1491-1495.

Park E, Nedo A, Caplan JL, et al. 2018. Plant-microbe interactions: organelles and the cytoskeleton in action. New Phytologist 217(3): 1012-1028.

Pathuri IP, Zellerhoff N, Schaffrath U, et al. 2008. Constitutively activated barley ROPs modulate epidermal cell size, defense reactions and interactions with fungal leaf pathogens. Plant Cell Reports 27(12): 1877-1887.

Peart JR, Lu R, Sadanandom A, et al. 2002. Ubiquitin ligase-associated protein SGT1 is required for host and nonhost disease resistance in plants. PNAS 99(16): 10865-10869.

Pecenkova T, Hala M, Kulich I, et al. 2011. The role for the exocyst complex subunits Exo70B2 and Exo70H1 in the plant-pathogen interaction. Journal of Experimental Botany 62(6): 2107-2116.

Pecenkova T, Markovic V, Sabol P, et al. 2017. Exocyst and autophagy-related membrane trafficking in plants. Journal of Experimental Botany 69(1): 47-57.

Ponce de Leon I, Oliver JP, Castro A, et al. 2007. Erwinia carotovora elicitors and Botrytis cinerea activate defense responses in Physcomitrella patens. BMC Plant Biology 7: doi: 10.1186/1471-2229-1187-1152.

Ponce de León I. 2011. The moss Physcomitrella patens as a model system to study interactions between plants and phytopathogenic fungi and oomycetes. Journal of Pathogens 2011: doi: 10.4061/2011/719873.

Ponce De Leon I, Schmelz EA, Gaggero C, et al. 2012. Physcomitrella patens activates reinforcement of the cell wall, programmed cell death and accumulation of evolutionary conserved defence signals, such as salicylic acid and 12-oxo-phytodienoic acid, but not jasmonic acid, upon Botrytis cinerea infection. Molecular Plant Pathology 13(8): 960-974.

Ponce de León I, Montesano M. 2013. Activation of defense mechanisms against pathogens in mosses and flowering plants. International Journal of Molecular Sciences 14(2): 3178-3200.

Ponce de Leon I, Montesano M. 2017. Adaptation mechanisms in the evolution of moss defenses to microbes. Frontiers in Plant Science 8: doi: 10.3389/fpls.2017.00366.

Poraty-Gavra L, Zimmermann P, Haigis S, et al. 2013. The Arabidopsis Rho of plants GTPase AtROP6 functions in developmental and pathogen response pathways. Plant Physiology 161(3): 1172-1188.

Porta H, Rocha-Sosa M. 2002. Plant lipoxygenases. Physiological and molecular features. Plant Physiolog 130(1): 15-21.

Porter K, Day B. 2016. From filaments to function: The role of the plant actin cytoskeleton in pathogen perception, signaling and immunity. Journal of Integrative Plant Biology 58(4): 299-311.

Qi J, Greb T. 2017. Cell polarity in plants: the Yin and Yang of cellular functions. Current Opinion in Plant Biology 35: $105-110$.

Qu S, Zhang X, Song Y, et al. 2017. THESEUS1 positively modulates plant defense responses against Botrytis cinerea through GUANINE EXCHANGE FACTOR4 signaling. Journal of Integrative Plant Biology 59(11): 797804.

Ratcliff F, Martin-Hernandez AM, Baulcombe DC. 2001. Technical Advance. Tobacco rattle virus as a vector for analysis of gene function by silencing. Plant Journal 25(2): 237-245.

Ravikumar R, Steiner A, Assaad FF. 2017. Multisubunit tethering complexes in higher plants. Curr Opin Plant Biol 40: 97-105. 
Rawat A, Brejskova L, Hala M, et al. 2017. The Physcomitrella patens exocyst subunit EXO70.3d has distinct roles in growth and development, and is essential for completion of the moss life cycle. New Phytologist 216(2): 438-454.

Reboledo G, Del Campo R, Alvarez A, et al. 2015. Physcomitrella patens activates defense responses against the pathogen Colletotrichum gloeosporioides. International Journal of Molecular Sciences 16(9): 22280-22298.

Reiner T, Hoefle C, Huesmann C, et al. 2015. The Arabidopsis ROP-activated receptor-like cytoplasmic kinase RLCK VI_A3 is involved in control of basal resistance to powdery mildew and trichome branching. Plant Cell Reports 34(3): 457-468.

Rensing SA, Lang D, Zimmer AD, et al. 2008. The Physcomitrella genome reveals evolutionary insights into the conquest of land by plants. Science 319(5859): 64-69.

Riedl J, Crevenna AH, Kessenbrock K, et al. 2008. Lifeact: a versatile marker to visualize F-actin. Nature Methods 5(7): 605-607.

Rivero C, Traubenik S, Zanetti ME, et al. 2017. Small GTPases in plant biotic interactions. Small GTPases: 1-11.

Robatzek S. 2007. Vesicle trafficking in plant immune responses. Cellular Microbiology 9(1): 1-8.

Rutter BD, Innes RW. 2017. Extracellular vesicles isolated from the leaf apoplast carry stress-response proteins. Plant Physiology 173(1): 728-741.

Rybak K, Steiner A, Synek L, et al. 2014. Plant cytokinesis is orchestrated by the sequential action of the TRAPPII and exocyst tethering complexes. Developmental Cell 29(5): 607-620.

Sabol P, Kulich I, Zarsky V. 2017. RIN4 recruits the exocyst subunit EXO70B1 to the plasma membrane. Journal of Experimental Botany 68: 3253-3265.

Safavian D, Zayed Y, Indriolo E, et al. 2015. RNA silencing of exocyst genes in the stigma impairs the acceptance of compatible pollen in Arabidopsis. Plant Physiology 169(4): 2526-2538.

Sagi M, Fluhr R. 2006. Production of reactive oxygen species by plant NADPH oxidases. Plant Physiology 141(2): 336-340.

Samuel MA, Chong YT, Haasen KE, et al. 2009. Cellular pathways regulating responses to compatible and selfincompatible pollen in Brassica and Arabidopsis stigmas intersect at Exo70A1, a putative component of the exocyst complex. Plant Cell 21(9): 2655-2671.

Sanderfoot A. 2007. Increases in the number of SNARE genes parallels the rise of multicellularity among the green plants. Plant Physiology 144(1): 6-17.

Sassmann S, Rodrigues C, Milne SW, et al. 2018. An immune-responsive cytoskeletal-plasma membrane feedback loop in plants. Current Biology 28(13): 2136-2144.

Schmelzer E. 2002. Cell polarization, a crucial process in fungal defence. Trends in Plant Science 7(9): 411-415.

Schmidt SM, Panstruga R. 2007. Cytoskeleton functions in plant-microbe interactions. Physiological and Molecular Plant Pathology 71(4-6): 135-148.

Schmidt SM, Kuhn H, Micali C, et al. 2014. Interaction of a Blumeria graminis f. sp. hordei effector candidate with a barley ARF-GAP suggests that host vesicle trafficking is a fungal pathogenicity target. Molecular Plant Pathology 15(6): 535-549.

Schoenaers S, Balcerowicz D, Vissenberg K 2017. Molecular mechanisms regulating root hair tip growth: a comparison with pollen tubes. In: Obermeyer G, Feijó J eds. Pollen tip growth: from biophysical aspects to systems biology. Cham: Springer International Publishing, 167-243.

Schultheiss H, Dechert C, Kogel KH, et al. 2002. A small GTP-binding host protein is required for entry of powdery mildew fungus into epidermal cells of barley. Plant Physiology 128(4): 1447-1454.

Schultheiss H, Dechert C, Kogel KH, et al. 2003. Functional analysis of barley RAC/ROP G-protein family members in susceptibility to the powdery mildew fungus. Plant Journal 36(5): 589-601.

Schultheiss H, Preuss J, Pircher T, et al. 2008. Barley RIC171 interacts with RACB in planta and supports entry of the powdery mildew fungus. Cellular Microbiology 10(9): 1815-1826.

Schutz I, Gus-Mayer S, Schmelzer E. 2006. Profilin and Rop GTPases are localized at infection sites of plant cells. Protoplasma 227(2-4): 229-235.

Sekeres J, Pejchar P, Santrucek J, et al. 2017. Analysis of exocyst subunit EXO70 family reveals distinct membrane domains in tobacco pollen tubes. Plant Physiology 173: 1659-1675.

Seo DH, Ahn MY, Park KY, et al. 2016. The N-terminal UND motif of the Arabidopsis U-box E3 ligase PUB18 is critical for the negative regulation of ABA-mediated stomatal movement and determines its ubiquitination specificity for exocyst subunit Exo70B1. Plant Cell 28(12): 2952-2973.

Shaw SL, Ehrhardt DW. 2013. Smaller, faster, brighter: advances in optical imaging of living plant cells. Annual Reviews of Plant Biology 64: 351-375. 
Shih HW, Miller ND, Dai C, et al. 2014. The receptor-like kinase FERONIA is required for mechanical signal transduction in Arabidopsis seedlings. Current Biology 24(16): 1887-1892.

Shimizu T, Nakano T, Takamizawa D, et al. 2010. Two LysM receptor molecules, CEBiP and OsCERK1, cooperatively regulate chitin elicitor signaling in rice. Plant Journal 64(2): 204-214.

Schindelin J, Arganda-Carreras I, Frise E, et al. 2012. Fiji: an open-source platform for biological-image analysis. Nature Methods 9: 676-682.

Shimono M, Lu YJ, Porter K, et al. 2016. The Pseudomonas syringae Type III effector HopG1 induces actin remodeling to promote symptom development and susceptibility during infection. Plant Physiology 171(3): 2239-2255.

Stassen JHM, van den Ackerveken G. 2011. How do oomycete effectors interfere with plant life? Current Opinion in Plant Biology 14: 407-414.

Stegmann M, Anderson RG, Ichimura K, et al. 2012. The ubiquitin ligase PUB22 targets a subunit of the exocyst complex required for PAMP-triggered responses in Arabidopsis. Plant Cell 24(11): 4703-4716.

Stegmann M, Anderson RG, Westphal L, et al. 2013. The exocyst subunit Exo70B1 is involved in the immune response of Arabidopsis thaliana to different pathogens and cell death. Plant Signaling and Behavior 8(12): doi: $10.4161 /$ psb.27421.

Stegmann M, Monaghan J, Smakowska-Luzan E, et al. 2017. The receptor kinase FER is a RALF-regulated scaffold controlling plant immune signaling. Science 355(6322): 287-289.

Stephan O, Cottier S, Fahlen S, et al. 2014. RISAP is a TGN-associated RAC5 effector regulating membrane traffic during polar cell growth in tobacco. Plant Cell 26(11): 4426-4447.

Sun H, Qiao Z, Chua KP, et al. 2018. Profilin negatively regulates formin-mediated actin assembly to modulate PAMP-triggered plant immunity. Current Biology 28(12): 1882-1895.

Sun J, Eklund DM, Montes-Rodriguez A, et al. 2015. In vivo Rac/Rop localization as well as interaction with RhoGAP and RhoGDI in tobacco pollen tubes: analysis by low-level expression of fluorescent fusion proteins and bimolecular fluorescence complementation. Plant Journal 84(1): 83-98.

Synek L, Schlager N, Elias M, et al. 2006. AtEXO70A1, a member of a family of putative exocyst subunits specifically expanded in land plants, is important for polar growth and plant development. Plant Journal 48(1): 54-72.

Synek L, Vukasinovic N, Kulich I, et al. 2017. EXO70C2 is a key regulatory factor for optimal tip growth of pollen. Plant Physiology 174(1): 223-240.

Takemoto D, Jones DA, Hardham AR. 2003. GFP-tagging of cell components reveals the dynamics of subcellular reorganization in response to infection of Arabidopsis by oomycete pathogens. Plant Journal 33(4): $775-792$.

Takemoto D, Hardham AR. 2004. The cytoskeleton as a regulator and target of biotic interactions in plants. Plant Physiology 136(4): 3864-3876.

Takemoto D, Jones DA, Hardham AR. 2006. Re-organization of the cytoskeleton and endoplasmic reticulum in the Arabidopsis pen 1-1 mutant inoculated with the non-adapted powdery mildew pathogen, Blumeria graminis f. sp. hordei. Molecular Plant Pathology 7(6): 553-563.

Takeuchi H, Higashiyama T. 2016. Tip-localized receptors control pollen tube growth and LURE sensing in Arabidopsis. Nature 531(7593): 245-248.

Takikawa Y, Kida S, Asayama F, et al. 2015. Defence responses of Aphanoregma patens (Hedw.) Lindb. to inoculation with Pythium aphanidermatum. Journal of Bryology 37(1): 1-7.

Tameling WI, Baulcombe DC. 2007. Physical association of the NB-LRR resistance protein Rx with a Ran GTPaseactivating protein is required for extreme resistance to Potato virus X. Plant Cell 19(5): 1682-1694.

Tan XJ, Thapa N, Sun Y, et al. 2015. A kinase-independent role for EGF receptor in autophagy initiation. Cell 160(12): 145-160.

Tan X, Feng Y, Liu Y, et al. 2016. Mutations in exocyst complex subunit SEC6 gene impaired polar auxin transport and PIN protein recycling in Arabidopsis primary root. Plant Science 250: 97-104.

Tang C, Deng L, Chang D, et al. 2016. TaADF3, an actin-depolymerizing factor, negatively modulates wheat resistance against Puccinia striiformis. Frontiers in Plant Science 6(1214): doi: 10.3389/fpls.2015.01214.

Tang H, De Keijzer J, Overdijk EJR, et al. 2019. Exocyst subunit Sec6 is positioned by microtubule overlaps in the moss phragmoplast prior to cell plate membrane arrival. Journal of Cell Science 132(3): doi: 10.1242/ jcs.222430.

Tanigaki Y, Ito K, Obuchi Y, et al. 2014. Physcomitrella patens has kinase-LRR R gene homologs and interacting proteins. PLoS One 9(4): doi: 10.3389/fpls.2015.01214.

TerBush DR, Novick P. 1995. Sec6, Sec8, and Sec15 are components of a multisubunit complex which localizes to small bud tips in Saccharomyces cerevisiae. Journal of Cell Biology 130(2): 299-312.

Thomma BP, Nurnberger T, Joosten MH. 2011. Of PAMPs and effectors: the blurred PTI-ETI dichotomy. Plant Cell 23(1): 4-15. 
Ting AE, Hazuka CD, Hsu SC, et al. 1995. rSec6 and rSec8, mammalian homologs of yeast proteins essential for secretion. PNAS 92(21): 9613-9617.

Tomczynska I, Stumpe M, Mauch F. 2018. A conserved RxLR effector interacts with host RABA-type GTPases to inhibit vesicle-mediated secretion of antimicrobial proteins. Plant Journal 95(2): 187-203.

Torres MA. 2010. ROS in biotic interactions. Physiologia Plantarum 138(4): 414-429.

Underwood W. 2016. Contributions of host cellular trafficking and organization to the outcomes of plantpathogen interactions. Seminars in Cell and Developmental Biology 56: 163-173.

van Gisbergen PA, Bezanilla M. 2013. Plant formins: membrane anchors for actin polymerization. Trends in Cellular Biology 23(5): 227-233.

van Gisbergen PAC, Wu SZ, Chang M, et al. 2018. An ancient Sec10-formin fusion provides insights into actinmediated regulation of exocytosis. Journal of Cell Biology 217(3): 945-957.

van Kan JAL. 2006. Licensed to kill: the lifestyle of a necrotrophic plant pathogen. Trends in Plant Science 11(5): 247-253.

van Ooijen G, van den Burg HA, Cornelissen BJ, et al. 2007. Structure and function of resistance proteins in solanaceous plants. Annual Review of Phytopathology 45: 43-72.

van Poppel PMJA, Guo J, van der Vondervoort PJIV, et al. 2008. The Phytophthora infestans avirulence gene Avr4 encodes an RXLR-dEER effector. Molecular Plant Microbe Interactions 21: 1460-1470.

Venus Y, Oelmuller R. 2013. Arabidopsis ROP1 and ROP6 influence germination time, root morphology, the formation of F-actin bundles, and symbiotic fungal interactions. Molecular Plant 6(3): 872-886.

Vidali L, Rounds CM, Hepler PK, et al. 2009. Lifeact-mEGFP reveals a dynamic apical F-actin network in tip growing plant cells. PLoS One 4(5): doi: 10.1371/journal.pone.0005744.

Vijn I, Govers F. 2003. Agrobacterium tumefaciens mediated transformation of the oomycete plant pathogen Phytophthora infestans. Molecular Plant Pathology 4(6): 459-467.

Viotti C, Bubeck J, Stierhof YD, et al. 2010. Endocytic and secretory traffic in Arabidopsis merge in the trans-Golgi network/early endosome, an independent and highly dynamic organelle. Plant Cell 22(4): 1344-1357.

Vleeshouwers VGAA, van Dooijeweert W, Keizer LCP, et al. 1999. A laboratory assay for Phytophthora infestans resistance in various Solanum species reflects the field situation. European Journal of Plant Pathology 105(3): 241-250.

Vleeshouwers VG, van Dooijeweert W, Govers F, et al. 2000. The hypersensitive response is associated with host and nonhost resistance to Phytophthora infestans. Planta 210(6): 853-864.

Voigt CA. 2014. Callose-mediated resistance to pathogenic intruders in plant defense-related papillae. Frontiers in Plant Science 5: doi: 10.3389/Fpls.2014.00168.

Voß S, Betz R, Heidt S, et al. 2018. RiCRN1, a crinkler effector from the arbuscular mycorrhizal fungus Rhizophagus irregularis, functions in arbuscule development. Frontiers in Microbiology 9(2068): doi: 10.1371/journal. pone.0005744.

Vukasinovic N, Cvrckova F, Elias M, et al. 2014. Dissecting a hidden gene duplication: the Arabidopsis thaliana SEC10 locus. PLoS One 9(4): doi: 10.1371/journal.pone.0094077.

Vukasinovic N, Zarsky V. 2016. Tethering complexes in the Arabidopsis endomembrane system. Frontiers in Cell and Developmental Biology 4: doi: 10.3389/fcell.2016.00046.

Vukasinovic N, Oda Y, Pejchar P, et al. 2017. Microtubule-dependent targeting of the exocyst complex is necessary for xylem development in Arabidopsis. New Phytologist 213(3): 1052-1067.

Wang D, Weaver ND, Kesarwani M, et al. 2005. Induction of protein secretory pathway is required for systemic acquired resistance. Science 308(5724): 1036-1040.

Wang W, Wen Y, Berkey R, et al. 2009. Specific targeting of the Arabidopsis resistance protein RPW8.2 to the interfacial membrane encasing the fungal haustorium renders broad-spectrum resistance to powdery mildew. Plant Cell 21(9): 2898-2913.

Wang JA, Ding Y, Wang JQ, et al. 2010. EXPO, an exocyst-positive organelle distinct from multivesicular endosomes and autophagosomes, mediates cytosol to cell wall exocytosis in Arabidopsis and tobacco cells. Plant Cell 22(12): 4009-4030.

Wang C, Zhu M, Duan L, et al. 2015. Lotus japonicus clathrin heavy Chain1 is associated with Rho-Like GTPase ROP6 and involved in nodule formation. Plant Physiology 167(4): 1497-1510.

Wang X, Chung KP, Lin W, et al. 2017. Protein secretion in plants: conventional and unconventional pathways and new techniques. Journal of Expimental Botany 69(1): 21-37.

Wang S, McLellan H, Bukharova T, et al. 2018. Phytophthora infestans RXLR effectors act in concert at diverse subcellular localisations to enhance host colonisation. Journal of Experimental Botany 70: 343-356. 
Wawra S, Trusch F, Matena A, Apostolakis K, Linne U, Zhukov I, Stanek J, Kozminski W, Davidson I, Secombes CJ, et al. 2017. The RxLR motif of the host targeting effector AVR3a of Phytophthora infestans is cleaved before secretion. The Plant Cell 29: 1184-1195.

Wen TJ, Hochholdinger F, Sauer M, et al. 2005. The roothairless1 gene of maize encodes a homolog of Sec3, which is involved in polar exocytosis. Plant Physiology 138(3): 1637-1643.

Wessling R, Epple P, Altmann S, et al. 2014. Convergent targeting of a common host protein-network by pathogen effectors from three kingdoms of life. Cell Host and Microbe 16(3): 364-375.

Whisson SC, Boevink PC, Moleleki L, et al. 2007. A translocation signal for delivery of oomycete effector proteins into host plant cells. Nature 450: 115-118.

Whisson SC, Boevink PC, Wang S, et al. 2016. The cell biology of late blight disease. Current Opinion in Microbiology 34: 127-135.

Wilson UE, Coffey MD. 1980. Cytological evaluation of general resistance to Phytophthora infestans in potato foliage. Annals of Botany 45(1): 81.

Wong HL, Pinontoan R, Hayashi K, et al. 2007. Regulation of rice NADPH oxidase by binding of Rac GTPase to its N-terminal extension. Plant Cell 19(12): 4022-4034.

Wu J, Tan X, Wu C, et al. 2013. Regulation of cytokinesis by exocyst subunit SEC6 and KEULE in Arabidopsis thaliana. Molecular Plant 6(6): 1863-1876.

Xue J-Y, Wang Y, Wu P, et al. 2012. A primary survey on bryophyte species reveals two novel classes of nucleotidebinding site (NBS) genes. PLoS One 7(5): doi: 10.1371/journal.pone.0036700.

Yamaguchi K, Imai K, Akamatsu A, et al. 2012. SWAP70 functions as a Rac/Rop guanine nucleotide-exchange factor in rice. Plant Journal 70(3): 389-397.

Yamada M, Miki T, Goshima G. 2016. Imaging mitosis in the moss Physcomitrella patens. In P. Chang \& R. Ohi, eds. Methods in Molecular Biology (Clifton, N.J.). New York, NY, United States: Springer New York, pp. 263-282.

Yamazaki A, Hayashi M. 2015. Building the interaction interfaces: host responses upon infection with microorganisms. Current Opinion in Plant Biology 23: 132-139.

Yan HQ, Zhang TT, Lan SC, et al. 2018. Ultrastructural study on the interaction between Physcomitrella patens and Botrytis cinerea. Plant Pathology 67: 42-50.

Yang Z. 2008. Cell polarity signaling in Arabidopsis. Annual Reviews of Cell and Developmental Biology 24: 551-575.

Yang Z, Liu J, Luo L, et al. 2018. The cotton GhRac6 gene encoding a plant ROP/RAC protein improves the plant defense response to aphid feeding. Plant Molecular Biology Reporter 36(5): 888-896.

Yi P, Goshima G. 2019. Fast, efficient, and precise gene editing in the moss Physcomitrella patens. bioRxiv: doi: $10.1101 / 643692$.

Yu F, Qian L, Nibau C, et al. 2012. FERONIA receptor kinase pathway suppresses abscisic acid signaling in Arabidopsis by activating ABI2 phosphatase. PNAS 109(36): 14693-14698.

Yun BW, Atkinson HA, Gaborit C, et al. 2003. Loss of actin cytoskeletal function and EDS1 activity, in combination, severely compromises non-host resistance in Arabidopsis against wheat powdery mildew. Plant Journal 34(6): 768-777.

Yun HS, Kwon C. 2017. Vesicle trafficking in plant immunity. Current Opinion in Plant Biology 40: 34-42.

Zarsky V, Kulich I, Fendrych M, et al. 2013. Exocyst complexes multiple functions in plant cells secretory pathways. Current Opinion in Plant Biology 16(6): 726-733.

Zhang Y, McCormick S. 2007. A distinct mechanism regulating a pollen-specific guanine nucleotide exchange factor for the small GTPase Rop in Arabidopsis thaliana. PNAS 104(47): 18830-18835.

Zhang Y, Liu CM, Emons AM, et al. 2010. The plant exocyst. Journal of Integrative Plant Biology 52(2): 138-146.

Zhang Y, Immink R, Liu CM, et al. 2013. The Arabidopsis exocyst subunit SEC3A is essential for embryo development and accumulates in transient puncta at the plasma membrane. New Phytologist 199(1): 74-88.

Zhang L, Kars I, Essenstam B, et al. 2014. Fungal endopolygalacturonases are recognized as microbeassociated molecular patterns by the Arabidopsis receptor-like protein RESPONSIVENESS TO BOTRYTIS POLYGALACTURONASES1. Plant Physiology 164(1): 352-364.

Zhang X, Pumplin N, Ivanov S, et al. 2015. EXO70I is required for development of a sub-domain of the periarbuscular membrane during arbuscular mycorrhizal symbiosis. Current Biology 25: 1-7.

Zhang B, Hua Y, Wang J, et al. 2017. TaADF4, an actin-depolymerizing factor from wheat, is required for resistance to the stripe rust pathogen Puccinia striiformis f. sp. tritici. Plant Journal 89(6): 1210-1224.

Zhao T, Rui L, Li J, et al. 2015. A truncated NLR protein, TIR-NBS2, is required for activated defense responses in the Exo70B1 mutant. PLoS Genetics 11(1): doi: 10.1371/journal.pgen.1004945.

Zhu X, Li S, Pan S, et al. 2018. CSI1, PATROL1, and exocyst complex cooperate in delivery of cellulose synthase complexes to the plasma membrane. PNAS 115(15): 3578-3587. 
Summary

Samenvatting

Acknowledgements

About the author

Publications

Education statement 



\section{Summary}

Plant diseases caused by pathogens threaten crop production worldwide. One of the approaches to combat pathogens is to improve the plant's own immunity, a strategy that requires detailed insight into the mechanisms underlying immunity. When plant cells are attacked by pathogens, their immune system is rapidly activated. One of the activated defence responses is a local and precise secretion of antimicrobial compounds and cell wall material at the site of attempted penetration. This requires polarized exocytosis. The central goal of this thesis is to understand polarized exocytosis in plant cells that are locally triggered by attack of a filamentous plant pathogen.

In the introduction (Chapter 1) we describe the current knowledge of the molecular machinery regulating exocytosis, and especially the role of the exocyst complex upon pathogen attack. The exocyst complex is a conserved multiprotein complex that facilitates one of the final steps of exocytosis. During exocytosis, exocytotic vesicles are transported to the plasma membrane where the exocyst complex mediates the tethering of these vesicles. Through this process, vesicles are tethered in the vicinity of the plasma membrane to allow vesicle fusion. In plants, the exocyst complex plays a role in many developmental processes that require polarized exocytosis, including cell division and tip growth. Increasing evidence shows that the exocyst complex is also important for plant immunity, which is supported by the fact that several plant pathogens produce effectors that target exocyst subunits.

In Chapter 2, we used the model plant Nicotiana benthamiana to study the role of exocyst subunits in defence against a range of pathogens. By silencing the genes encoding exocyst subunits we showed that several exocyst subunits are required for proper plant defence against the (hemi)biotrophic plant pathogens Phytophthora infestans and Pseudomonas syringae. In contrast, some subunits seem to act as susceptibility factor for the necrotrophic pathogen Botrytis cinerea. In addition, most exocyst subunits were found to play a role in callose deposition. Together, this is a strong indication that the exocyst complex functions in mediating basal plant immunity against different unrelated pathogens.

To investigate the function of the exocyst complex during plant defence at a cellular level, we established a novel model system using the moss Physcomitrella patens and the oomycete pathogen Phytophthora (Chapter 3). High resolution live-cell imaging of plant-microbe interactions is often challenged by the multi-layered nature of plant organs. To circumvent this, we used protonema cells of $P$. patens which have a single celled architecture that is ideal for microscopy. For this study we developed a special imaging set-up and demonstrated the suitability of this set-up to visualize cellular defence responses, such as actin cytoskeletal rearrangements. We tested several Phytophthora species for their ability to infect $P$. patens and showed that two species, $P$. infestans and $P$. capsici, frequently and successfully penetrate moss cells and grow intracellularly. Occasionally penetration attempts were halted by deposition of cell wall material in papilla-like structures, a phenomenon that is common in seed plants. Infection also 
caused upregulation of defence-related gene expression, and all together, this shows that Phytophthora infection induces general defence responses in P. patens. In Chapter $\mathbf{4}$ and Chapter 5, this novel model system is used to study the subcellular behaviour of exocyst subunits during plant defence in $P$. patens and the effect of $P$. infestans effector AVR1 on exocyst complex function, respectively.

As demonstrated by us (Chapter 2) and others in studies using gene silencing or knock-out mutants, the exocyst complex is important for plant immunity. Defects in exocyst functioning cause, depending on the affected subunit, developmental defects of varying severity. One could argue that these defects are the primary cause of the impaired defence. In the case of a direct effect of the exocyst complex on plant immunity, it is hypothesized that the exocyst complex is recruited to sites of pathogen attack to facilitate defence-related vesicle tethering. In Chapter 4, we tested this hypothesis by monitoring the subcellular localization of exocyst subunits during infection of $P$. patens by $P$. capsici. We made use of $P$. patens lines expressing fluorescently-tagged exocyst subunits and one expressing fluorescently-tagged Vamp72A1, a marker for defencerelated vesicles. However, the deposition of highly autofluorescent material in papillalike structures and in hyphal encasements surrounding invasive hyphae hampered the visualisation of the low abundant exocyst subunits and Vamp72A1. To circumvent this we made use of time-gated confocal microscopy and demonstrated that exocyst subunits Sec $3 a$, Sec5b, Sec5d and Sec6 are recruited to sites of attempted pathogen penetration, even before autofluorescent material is deposited. Upon pathogen invasion, these exocyst subunits accumulated on the membrane surrounding papilla-like structures and hyphal encasements. In addition, the marker Vamp72A1-accumulated in the cytoplasm surrounding infection sites. Altogether, these results suggest that the exocyst complex is directly involved in facilitating polarized exocytosis to infection sites.

Pathogens exploit effectors to suppress plant defence processes. Several of these effectors are known to target the membrane trafficking machinery amongst others by interacting with subunits of the exocyst complex. In Chapter 5, we focused on AVR1 from P. infestans, an RXLR effector that was previously shown to bind to the potato exocyst subunit Sec5 and to suppress callose deposition. So far, the exact mechanism by which AVR1 manipulates Sec5 functioning is unknown. We first tested the ability of AVR1 to bind to Sec5 homologs of $P$. patens. In a yeast-two-hybrid assay, AVR1 interacted with PpSec5b and PpSec5d, whereas AVR1 ${ }^{\Delta T}$, a truncated version of AVR1, did not. To study the effect of AVR1 on $P$. patens growth, we generated transgenic $P$. patens lines carrying $\beta$-estradiol inducible $A V R 1$ or $A V R 1^{\Delta \top}$ transgenes. $A V R 1$ or $A V R 1^{\Delta T}$ expressing lines showed defects in the development of protonema cells and a minor reduction in growth. This phenotype was similar to the phenotypes observed in Sec5- or Sec6-silenced lines, suggesting that exocyst functioning is impaired by AVR1 and AVR1 ${ }^{\Delta T}$. On a subcellular level, the presence of AVR1 or AVR1 ${ }^{\Delta T}$ did not seem to alter the localization of Sec5b or Sec5d. Therefore, we propose that both AVR1 and AVR1 ${ }^{\Delta T}$ affect Sec5 functioning in P. patens, without detectable alteration of its subcellular localization. 
Overall, the research described in this thesis demonstrates the power of a multidisciplinary approach for unravelling plant-pathogen interactions. The data obtained by combining phytopathological and cell biological approaches show that the exocyst complex is an important part of plant immunity and that exocyst subunits are recruited to sites of pathogen infection. In Chapter 6, I discuss these findings in a broader perspective and propose a model for the molecular regulation of polarized exocytosis during plant defence. I hypothesize that signalling pathways mediated by Rho family small GTPases orchestrate polarized exocytosis in response to both pathogenic and beneficial microbes. 


\section{Samenvatting}

Plantenziekten veroorzaakt door schimmels, bacteriën en andere microben zijn een groot probleem in de land- en tuinbouw en zorgen jaarlijks voor grote oogstverliezen. Een mogelijke oplossing is de natuurlijke afweer van planten te verbeteren. Dit vereist inzicht in hoe planten zich beschermen tegen ziekteverwekkers en hoe ziekteverwekkers planten ziek maken. Als een plantencel wordt aangevallen door een ziekteverwekker wordt het afweersysteem geactiveerd. Als onderdeel van deze afweerreactie scheidt de plantencel stoffen uit die de groei van de ziekteverwekker kunnen remmen en zet de plantencel celwandmateriaal af op de plek waar de ziekteverwekker probeert binnen te dringen. Dit vereist gerichte exocytose. Exocytose is een proces waarbij met behulp van membraanblaasjes, zogeheten vesikels, stoffen uit worden gescheiden in het extracellulaire milieu. Het doel van het onderzoek beschreven in dit proefschrift is meer inzicht te krijgen in gerichte exocytose in cellen die aangevallen worden door ziekteverwekkers.

In de introductie (Hoofdstuk 1) geven we een overzicht van de huidige kennis van de moleculaire mechanismen die exocytose reguleren tijdens afweer tegen ziekteverwekkers, en met name de rol van het exocystcomplex in dit proces. Het exocystcomplex is een geconserveerd eiwitcomplex dat bestaat uit verschillende eiwitten, zogeheten subeenheden, en dat één van de laatste stappen van de exocytose ondersteunt. Tijdens exocytose worden vesikels naar het celmembraan getransporteerd en door het exocystcomplex dicht genoeg bij het celmembraan gebracht om er uiteindelijk mee te versmelten. In plantencellen speelt het exocystcomplex een belangrijke rol tijdens ontwikkelingsprocessen die afhankelijk zijn van gerichte exocytose, zoals bijvoorbeeld celdeling en celgroei. Er is daarnaast steeds meer bewijs dat het exocystcomplex ook nodig is tijdens de afweer tegen ziekteverwekkers. Dit wordt ondersteund door het feit dat een aantal ziekteverwekkers effectoreiwitten produceren die binden aan exocystsubeenheden.

Als eerste stap (Hoofdstuk 2) hebben we de tabaksplant Nicotiana benthamiana gebruikt om de rol van exocystsubeenheden te bestuderen tijdens de afweer tegen verschillende ziekteverwekkers. Door de expressie van de coderende genen te onderdrukken, konden we aantonen dat een aantal exocystsubeenheden essentieel zijn voor een effectieve verdediging tegen twee (hemi)biotrofe ziekteverwekkers: de oömyceet Phytophthora infestans die de aardappelziekte veroorzaakt, en de bacterie Pseudomonas syringae. Daarentegen waren er ook een aantal exocystsubeenheden die juist de vatbaarheid voor de necrotrofe schimmel Botrytis cinerea verhoogden. Verder bleek dat de meeste exocystsubeenheden een rol spelen bij de afzetting van callose, celwandmateriaal dat wordt gevormd als plantencellen worden aangevallen door ziekteverwerkers. Dit wijst erop dat het exocystcomplex in planten een functie heeft in de basale afweer tegen ziekteverwekkers. 
Om deze functie op celniveau te kunnen onderzoeken, hebben we een nieuw modelsysteem ontwikkeld met het mos Physcomitrella patens als waardplant en Phytophthora als ziekteverwekker (Hoofdstuk 3). Het visualiseren van een interactie tussen plant en ziekteverwekker op celniveau en met hoge resolutie wordt vaak bemoeilijkt door het feit dat plantenorganen uit meerdere cellagen bestaan. Protonemacellen van P. patens daarentegen bestaan slechts uit één cellaag en zijn daardoor ideaal voor microscopie. Met een speciaal hiervoor ontwikkelde microscoop opstelling konden we afweerreacties in protonemacellen op celniveau visualiseren en de reorganisatie van het actinecytoskelet op de infectieplaats in beeld te brengen. Van de geteste Phytophthora soorten bleken $P$. infestans en $P$. capsici in staat te zijn om succesvol moscellen binnen te dringen en te infecteren. Soms werd de Phytophthora aanval door het mos tegengehouden door het afzetten van celwandmateriaal in zogenaamde papilla-achtige structuren. Deze afweerstructuren worden ook vaak aangetroffen in zaadplanten. Ook leidde infectie tot een hogere expressie van afweer-gerelateerde genen. Dit alles laat zien dat Phytophthora infectie basale afweerreacties activeert in het mos $P$. patens. In Hoofdstuk 4 en Hoofdstuk 5 wordt dit nieuwe modelsysteem gebruikt om het subcellulaire gedrag van exocystsubeenheden tijdens afweer te bestuderen alsook het effect van het $P$. infestans effectoreiwit AVR1 op het functioneren van het exocystcomplex.

Zo aangetoond in Hoofdstuk 2 en ook in studies van andere onderzoekers, heeft het exocystcomplex een functie in de basale afweer tegen ziekteverwekkers. Een slecht functionerend exocystcomplex veroorzaakt echter ook ontwikkelings-gerelateerde afwijkingen en die variëren afhankelijk van de exocystsubeenheid die gedereguleerd is. Om uit te sluiten dat dat deze ontwikkelings-gerelateerde afwijkingen de oorzaak zijn van de verminderde afweer, hebben we in Hoofdstuk 4 de subcellulaire lokalisatie van exocystsubeenheden tijdens infectie met behulp van microscopie bestudeerd. Als immers het exocystcomplex direct betrokken is bij de afweer, dan bevindt het complex zich zeer waarschijnlijk op de plaats waar een ziekteverwekker de cel aanvalt om daar afweergerelateerde vesikels dicht bij het celmembraan te brengen. We hebben deze hypothese getest door gebruik te maken van een viertal moslijnen met fluorescerend gelabelde exocystsubeenheden en één moslijn met een fluorescente Vamp72A1 eiwitmarker die afweer-gerelateerde vesikels markeert. Tijdens infectie van deze moslijnen met $P$. capsici werd echter sterk auto-fluorescerend materiaal afgezet in papilla-achtige structuren en rondom intracellulair groeiende hyfen van de ziekteverwekker hetgeen een belemmering vormde voor het zichtbaar maken van de exocystsubeenheden. Om de autofluorescentie te vermijden hebben we gebruik gemaakt van time-gated confocale fluorescentie microscopie en konden zo aantonen dat de exocystsubeenheden Sec3a, Sec5b, Sec5d en Sec6 inderdaad aanwezig zijn op de plaats waar de ziekteverwekker de cel binnen probeert te dringen. De subeenheden waren al aanwezig voordat het auto-fluorescerende materiaal werd afgezet. Later tijdens het infectieproces accumuleerden deze exocystsubeenheden op het membraan rondom papilla-achtige structuren en rond intracellulair groeiende hyfen van de ziekteverwekker terwijl de vesikel-marker Vamp72A1 accumuleerde in het cytoplasma dicht bij de plaats waar de cel werd aangevallen door de ziekteverwekker. 
Deze bevindingen suggereren dat het exocystcomplex direct betrokken is bij het ondersteunen van gerichte exocytose naar de plaats waar de cel wordt aangevallen.

Ziekteverwekkers gebruiken effectoreiwitten om afweer van de plant te onderdrukken. Van een aantal van deze effectoreiwitten is bekend dat ze in de plantencel terechtkomen en daar binden aan planteneiwitten die betrokken zijn bij het transport van membranen, waaronder subeenheden van het exocystcomplex. AVR1 is een effectoreiwit van de ziekteverwekker $P$. infestans, waarvan al eerder is aangetoond dat het bindt aan de aardappel exocystsubeenheid Sec5 en daardoor de afzetting van callose onderdrukt. Het exacte mechanisme waarmee AVR1 het functioneren van Sec5 beïnvloedt, is nog onbekend. In Hoofdstuk 5 hebben we eerst getest of AVR1 ook kan binden aan Sec5 eiwitten van het mos $P$. patens. In een 'two-hybrid' test in gist, bleek dat dat AVR1 kan binden aan PpSec5b en PpSec5d, terwijl AVR1 ${ }^{\Delta T}$, een gemuteerde versie van AVR1, dat niet kan. Om het effect van AVR1 op de groei van P. patens te bestuderen, hebben we transgene $P$. patens lijnen gegenereerd waarin we de aanmaak van de eiwitten AVR1 of AVR1 ${ }^{\Delta T}$ met behulp van het hormoon $\beta$-estradiol kunnen reguleren. In moslijnen waarin de aanmaak van AVR1 of AVR1 ${ }^{\Delta T}$ werd geïnduceerd, trad groeiremming op en was de ontwikkeling van protonemacellen verstoord. Dit fenotype was vergelijkbaar met de fenotypes die werden waargenomen in moslijnen waarin de hoeveelheid exocystsubeenheid Sec5 of Sec6 was gereduceerd. Hoewel dit suggereert dat het functioneren van het exocystcomplex wordt verstoord door de aanwezigheid van AVR1 en AVR1 ${ }^{\Delta T}$, bleek er op celniveau geen effect te zijn op de lokalisatie van Sec5b of Sec5d. Blijkbaar gaat het negatieve effect van zowel AVR1 als AVR1 ${ }^{\Delta T}$ op Sec5 in het mos P. patens, niet gepaard met een waarneembare verandering in de subcellulaire lokalisatie van deze exocystsubeenheid.

Het onderzoek dat in dit proefschrift wordt beschreven toont de kracht van een multidisciplinaire aanpak aan voor het ontrafelen van interacties tussen planten en hun ziekteverwekkers. De resultaten, verkregen door een combinatie van een fytopathologische en celbiologische aanpak, laten zien dat het exocystcomplex een belangrijk onderdeel is van het afweersysteem van een plant en dat exocystsubeenheden zich bevinden op de plaats waar de cel wordt aangevallen. In hoofdstuk 6 bespreek ik deze bevindingen in een breder perspectief en presenteer een model van de moleculaire regulatie van gerichte exocytose tijdens afweerreacties. Hierin staan eiwitten uit de familie van kleine Rho GTPases centraal en ik veronderstel dat dit model niet alleen representatief is voor interacties met ziekteverwekkers, maar ook voor interacties met goedaardige micro-organismen. 


\section{Acknowledgements}

It is a great pleasure that my $\mathrm{PhD}$ thesis is now finished. It was an illuminating journey during which I learned many great skills and got support from many great people. I will try to thank all of those here.

First of all, my supervisors, from which I learned the most. Francine, thank you for your care and support from the first day we met. Thanks for all our productive discussions, your enthusiasm, ideas and your thorough and useful feedback, especially during the writing process. Tijs, we met even earlier, when you supervised my bachelor thesis. Thank you for all your constructive feedback. Thanks for your endless optimism, encouragement and guidance. It is amazing that your door is always open and you always made time for me. Klaas, thank you for our many great talks and for being a friend in the lab. You were always available for help: from practical set-ups in the lab - you knew a practical solution to every problem - to personal advice when needed most.

Then my lab-mates, with whom I spend countless hours in the lab, greenhouse, microscoperoom or flow cabinet. Han, thank you for all your help and collaboration. All the moss lines we created together, the endless transformations and genotyping. And the yeast-twohybrid experiments you performed for me when I was on pregnancy leave. I wish you all the best with finishing your PhD and finding a great job close to your husband. Jeroen, thank you for setting up the moss system in the Cell Biology lab, for teaching me the moss work and helping me with my experiments. I wish you all the best for the future. Yu, I started when you were finishing your PhD. I want to thank you for our collaboration and for explaining me the silencing work and disease assays. Your help gave me a flying start. I wish you all the best in China. Thank you Chara for our friendship and our great talks. Also many thanks for your help with cultivating the Phytophthora species. I wish you all the best with Manos in the west.

I also want to thank the other people from the laboratories I worked in. For the nice chats during lunches and coffee-breaks, help with experiments and feedback and helpful suggestions during meetings. Form the Cell Biology lab: Kris, Alejandra, Peter, Juliane, Aniek, Olja, Henk, Norbert, Andre, Otto, Marie-Jose and Marcel. Hannie, thank you for your career-related advices. From the Phytophthora group: Johan, Sander, Jeroen, Yan, Shuqing, Kiki, Harold, Rob and Nathalie. And from the rest of the Phytopathology lab: Mireille, Hanna, Jordi, Scott, Aranka, Martin, Xiaoqian, Nick, Katharina, Jasper, Malaika, Maikel, Jasper, Hui, Jinbin, Jinling, Ester, Grardy, Laurens, Ali, Bart, Matthieu, Jan, Michael, Gert and others. Thank you all! In addition, I would like to express my gratitude to the people from Unifarm, especially Henk and Bert, for taking excellent care of my plants in the greenhouse.

Then the BSc and MSc students that I supervised during their theses: Vera, Joep, Deborah, Wouter, Ruth, Wouter, Daniël, Raimo and Roel. Thank you for your company, your help and contributions in the lab - several of your results are part of this thesis - and also many thanks for making me aware of my passion for teaching and coaching students. 
I would also like to thank my fellow EPS PhD council members, Hanna, Setareh, Jordi, Kris, Lot, Lotte, Amalia and others, with whom I enjoyed organizing the first Get2Gether for EPS PhD students. In addition, I would like to thank Susan, Douwe and Ingrid from the EPS Graduate School for their support.

Iliana, thank you for creating the beautiful cover and layout of this book!

Tijdens mijn PhD had ik het voorrecht om gastcolleges te geven op een aantal hogescholen. Heel veel dank aan hogeschooldocenten Frans, Carole, Anna, Tanja, Robert Jan en Kees, voor jullie vertrouwen in mij en de kansen die jullie mij gegeven hebben om onderwijservaring op te doen. Hopelijk komen we elkaar in de toekomst nog vaak tegen!

Lieve mede-moeder-PhD-ers Sabine en Sara. Het is zo fijn en belangrijk geweest om alle moederschap struggles in combinatie met het doen van een $\mathrm{PhD}$ met jullie te kunnen delen. Lieve Sabine, bedankt voor je vriendschap en het zijn van mijn paranimf! Ik wens je heel veel succes met het afronden van je eigen $\mathrm{PhD}$ en heel veel geluk met je prachtige mannen.

Dan mijn zangvrienden van Canzone: Jos, Janna, Wouter, Joost en Sjoerd. Bedankt voor al het heerlijke en ontspannende samen zingen, samen kletsen en samen eten. Hopelijk kunnen we dat nog lang blijven doen!

Lieve Claire \& Michael, Nadia \& Maurits en Sanne \& Wouter. Bedankt voor jullie vriendschap en alle heerlijke, gezellige momenten samen met onze kinderen. Lieve Albertine, bedankt voor je vriendschap, onze fantastische gesprekken, alle woensdagen die we samen doorbrachten met onze kinderen en het feit dat ik altijd op je hulp kan rekenen.

Lieve Janna, we ontmoetten elkaar 11 jaar geleden op een Open Dag voor de studie Biologie in Wageningen en zijn elkaar daarna nooit meer uit het oog verloren. We hebben veel samen gezongen en georganiseerd. Ik heb je doorzettingsvermogen en kracht altijd bewonderd. Bedankt voor al je advies en hulp, van het oppassen op onze kinderen tot het zijn van paranimf op deze belangrijke dag. Heel veel succes en geluk met je carrière in de wetenschap en je persoonlijke leven!

Ook veel dankaan mijn lieve zus en broers, Angela, Manuel, Andreas en Christian. Bedankt voor al onze gezellige familiedagen, heerlijk kanoën in de zon, Sinterklaasgedichten voorlezen en gezellig samen koken. Op nog heel veel mooie momenten samen!

Papa, ik wil je bedanken voor je steun en aanmoediging. Het is fijn om te weten dat je trots op me bent.

Mama en Ludo, bedankt voor alle heerlijke ontspannende weekenden in Alkmaar, het vele oppassen op onze kinderen en jullie interesse en luisterend oor. Ludo, succes met het afronden van je eigen schrijfproject! Lieve mama, bedankt voor je onvoorwaardelijke liefde en steun. Bedankt voor alles, ik hou van je! 
Lieve Evan en Linde, jullie zijn mijn alles! Bedankt voor alle afleiding, van slapeloze nachten tot brandweertje spelen en zandtaartjes bakken in de tuin. Door jullie kon ik alles altijd relativeren en in het juiste perspectief plaatsen. Ik hou van jullie!

Bedankt lieve Bram, zonder je enorme liefde, geduld, hulp en zorg voor de kinderen was het schrijven van dit boekje nooit mogelijk geweest. Bedankt voor al je steun en dat je er altijd voor me bent. Bij jou kan ik helemaal mezelf zijn en tot rust komen. Samen kunnen we alles aan! Ik hou heel veel van je!

Liefs, Elysa 


\section{About the author}

Elysa Overdijk was born on August $14^{\text {th }}, 1989$ in Zeddam, the Netherlands. In 2008 she graduated from high school and obtained her VWO diploma with distinction. Her passion for nature and plants brought her to Wageningen where she studied biology.

After obtaining her BSc degree (2011, cum laude), Elysa choose to further specialize in cell and molecular plant biology. She performed her first MSc thesis at the Laboratory of Cell Biology on the regulation of secondary cell wall deposition during xylem formation. She then focussed her attention

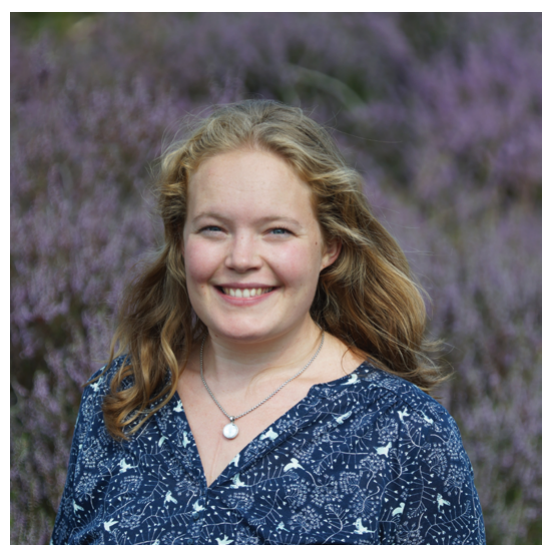
on plant cells that are attacked by pathogens. During her second MSc thesis at the Laboratory of Phytopathology, she set-up a novel pathosystem using the moss Physcomitrella patens and Phytophthora pathogens to visualize cellular plant defence. This model system was the basis of a PhD project proposal she wrote as MSc student in the framework of a Graduate Program Course offered by the Graduate School Experimental Plant Sciences. Elysa was one of the four students that year that were selected to receive a personal grant for a PhD project from the Dutch Research Council (NWO).

After her graduation as MSc in Biology (2014, cum laude), Elysa started her PhD research in the laboratories of Cell Biology and Phytopathology at Wageningen University where she was supervised by Francine Govers, Tijs Ketelaar and Klaas Bouwmeester. The results of this research are described in this thesis. As member of the PhD council of the Graduate School Experimental Plant Sciences, Elysa was involved in organizing amongst others the Get2Gether days for PhD students.

Elysa's hobby is classical singing and she likes teaching. During her study she was a member of the Wageningen Student Choir and Orchestra Association (WSKOV) and was active amongst others, in the organizing committee of a concert tour in Sweden. As teacher, Elysa has been active as student assistant in BSc courses for students in plant sciences and cell biology and she guided excursions for students taking the Dutch Flora course. During her PhD, Elysa enjoyed supervising BSc and MSc students. She further expanded her teaching skills by giving guest lectures on plant immunity at Dutch Universities of Applied Sciences (Hogescholen) and she also developed a practical module on plant diseases for the HAN University of Applied Sciences. In September 2019, Elysa was appointed as a lecturer Green Biotechnology at the Inholland University of Applied Sciences in Amsterdam and in this position she can combine her love for molecular plant sciences and teaching.

Elysa is married with Bram and they have a son and a daughter. She enjoys singing with her friends in the a capella ensemble Canzone and harvesting vegetables with her kids in the organic community garden. 


\section{Publications}

Overdijk EJR, Putker V, Smits J, Tang H, Bouwmeester K, Govers F, Ketelaar T. Phytophthora infestans RXLR effector AVR1 disturbs the growth of Physcomitrella patens, without affecting Sec5 localization. In preparation.

Overdijk EJR, Tang H, Borst JW, Govers F, Ketelaar T. Time-gated confocal microscopy reveals accumulation of plant exocyst subunits at the plant-pathogen interface. Submitted.

Tang H, de Keijzer J, Overdijk EJR, Sweep E, Steentjes M, Vermeer J, Janson M, Ketelaar T. 2019. Exocyst subunit Sec6 is positioned by microtubule overlaps in the moss phragmoplast prior to the arrival of cell plate membrane. Journal of Cell Science doi: $10.1242 / j c s .222430$.

Du Y," Overdijk EJR," Berg JA, Govers F, Bouwmeester K. 2018. Solanaceous exocyst subunits are involved in immunity to diverse plant pathogens. Journal of Experimental Botany 62 (3): 655-666.

\# equal contribution

Rose LE, Overdijk EJR, van Damme M. 2018. Small RNA molecules and their role in plant disease. European Journal of Plant Pathology doi:10.1007/s10658-018-01614-w.

Griffiths SA, Cox RJ, Overdijk EJR, Mesarich CH, Saccomanno B, Lazarus CM, de Wit PJGM, Collemare J. 2018. Assignment of a dubious gene cluster to melanin biosynthesis in the tomato fungal pathogen Cladosporium fulvum. PLoS ONE 13(12): e0209600.

Griffiths S, Mesarich CH, Overdijk EJR, Saccomanno B, de Wit PJGM, Collemare J. 2018. Down-regulation of cladofulvin biosynthesis is required for biotrophic growth of Cladosporium fulvum on tomato. Molecular Plant Pathology 19: 369-380.

Overdijk EJR, De Keijzer J, De Groot D, Schoina C, Bouwmeester K, Ketelaar T, Govers F. 2016. Interaction between the moss Physcomitrella patens and Phytophthora: a novel pathosystem for live-cell imaging of subcellular defence. Journal of Microscopy 263: 171-180.

Akkerman M, Overdijk EJR, Schel JHN, Emons AMC, Ketelaar T. 2011. Golgi body motility in the plant cell cortex correlates with actin cytoskeleton organization. Plant Cell Physiology 52 (10): 1844-1855. 


\title{
Education Statement of the Graduate School \\ Experimental Plant Sciences
}

\author{
Issued to: Elysa J.R. Overdijk \\ Date: 18 October 2019 \\ Group: Laboratory of Phytopathology \\ University: Wageningen University \& Research
}

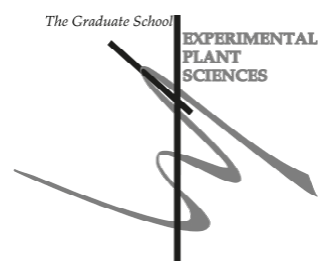

\begin{tabular}{|c|c|c|}
\hline $\begin{array}{l}\text { 1) Start-Up Phase } \\
\text { First presentation of your project }\end{array}$ & date & $\underline{c p}$ \\
\hline $\begin{array}{l}\text { The plant exocyst as an expeller of pathogens } \\
\text { Writing or rewriting a project proposal } \\
\text { Writing a review or book chapter } \\
\text { MSc courses }\end{array}$ & 16 Jun 2014 & 1.5 \\
\hline
\end{tabular}

\section{2) Scientific Exposure}

$\begin{array}{cc}\underline{\text { date }} & \underline{c p} \\ \text { 29-30 Jan 2015 } & 0.6 \\ \text { 09 Feb 2017 } & 0.3 \\ \text { 11 Feb 2019 } & 0.3 \\ \text { 08 Jan 2015 } & 0.3 \\ \text { 20 Feb 2015 } & 0.3 \\ \text { 23 Jan 2017 } & 0.3\end{array}$

- Lunteren Days and other national platforms

Annual meeting 'Experimental Plant Sciences', Lunteren, The

14-15 Apr $2014 \quad 0.6$ Netherlands

Annual meeting 'Experimental Plant Sciences', Lunteren, The

13-14 Apr $2015 \quad 0.6$ Netherlands

Annual meeting 'Experimental Plant Sciences', Lunteren, The

10-11 Apr 2017 Netherlands

Annual meeting 'Experimental Plant Sciences', Lunteren, The Netherlands

- Seminars (series), workshops and symposia

Seminar Sophien Kamoun, Genome and effector evolution in the Irish

28 May 2014 potato famine pathogen lineage

Seminar Joy Bergelson, Maintaining an ancient balanced polymorphism for resistance amidst diffuse interactions 
Seminar George Coupland, Seasonal flowering in annual and

19 Jan 2015

0.1

perennial plants

Seminar Monica Höfte, Understanding rice brown spot, a disease

6 Feb 2015

0.1

induced by physiological stress

Seminar Gero Steinberg, Long-distance endosome trafficking drives

5 Jun 2015

0.1

fungal effector production during plant infection

Seminar Caitilyn Allen, How Ralstonia solanacearum succeeds in plant

29 Apr 2016

0.1

xylem vessels

Seminar Jill Harrison, How did branching plant bodies evolve?

12 May 2016

0.1

Seminar Edze Westra and Jennifer Doudna, Rewriting our genes

30 Sep 2016

0.2

Seminar Daniel von Wangenheim, Lightsheet microscopy in plants

11 Oct 2016

0.1

Seminar Hans Thordal-Christensen, Membrane trafficking in plant cells

12 Dec 2016

0.1

attacked by powdery mildew fungi

Seminar Victor Jones, The genetic control of rhizoid development in

18 Apr 2017

0.1 the liverwort Marchantia polymorpha

Seminar Gerd Jürgens, Membrane traffic and fusion in plant

11 May 2017

0.1

cytokinesis - an evolutionary perspective

Seminar Magdalena Bezanilla, Cytoskeletal crosstalk impacts cell

6 Nov 2017

0.1

shape and development

Seminar Volker Lipka, Arabidopsis interactions with fungal pathogens

14 Sept 2018

0.1

Seminar Guido van der Ackerveken, Why are my leafy greens having

8 Mar 2019

0.1

these downy mildews?

Seminar Nick Talbot, Investigating the biology of plant infection by

2 Apr 2019

0.1

rice blast fungus

Netwerkdag TKI Uitgangsmaterialen, Zeist, The Netherlands

12 May 2014

0.2

DSCB Trippenhuis meeting: Vesicles on the Move: The cytoskeletal

17 Oct 2014

0.3 connection, Amsterdam, The Netherlands

Phytopathology Mini symposium, Wageningen, The Netherlands

WPC PhD Symposium, Wageningen, The Netherlands

Symposium Translational Plant \& Microbial Biology, Utrecht, The Netherlands

24 Nov 2014

0.2

6 May $2016 \quad 0.3$

9 May 2017

\section{- Seminar plus}

- International symposia and congresses

COST Workshop on cellular dynamics of effector action and

9-11 Apr 2014

recognition, Toulouse, France

Botanical Microscopy Meeting, Exeter, UK

20-23 Apr 2015

1.2

New Phytologist Symposium: Cell biology at the plant-microbe interface, Munich, Germany

29 Nov- 1 Dec

0.9

2015

Annual Meeting Society of Experimental Biology - Imaging plant

3-6 Jul 2017 


\section{Presentations}

Talk: The plant exocyst as an expeller of pathogens, COST Workshop, $\quad 9$ Apr $2014 \quad 1.0$ Toulouse, France

Talk: Is de exocyst in planten een exorcist van pathogenen?

12 May $2014 \quad 1.0$

Netwerkdag TKI uitgangsmaterialen, Zeist, The Netherlands

Talk: Novel Phytophthora-Physcomitrella pathosystem gives insight

23 Apr $2015 \quad 1.0$

into cellular plant defence responses, Botanical Microscopy Meeting,

Exeter, UK

Talk: Cell Biology and Phytopathology, Meeting BU Biointeractions \&

10 Feb $2015 \quad 1.0$

Plant Health, Wageningen, The Netherlands

Talk: Effector AVR1 targets plant exocyst subunit Sec5 to suppress

immunity, Annual Meeting Society of Experimental Biology,

Gothenburg, Sweden

Talk: Effector AVR1 targets plant exocyst subunit Sec5 to suppress

29 Aug $2017 \quad 1.0$

immunity, Summer School Environmental Signalling in Plants, Utrecht,

The Netherlands

Talk: The vesicle-tethering exocyst complex polarizes during plant

9 Apr $2019 \quad 1.0$

defence, Annual meeting 'Experimental Plant Sciences', Lunteren, The

Netherlands

Poster: The plant exocyst as an expeller of pathogens, Annual meeting

'Experimental Plant Sciences', Lunteren, The Netherlands

Poster \& Flash talk: The Physcomitrella-Phytophthora interaction, New

3 Jul $2017 \quad 1.0$

Phytologist Symposium, Munich, Germany

Poster: Interaction between the moss Physcomitrella and Phytophthora, 30 Nov $2015 \quad 1.0$

New Phytologist Symposium, Munich, Germany

Poster: Effector AVR1 targets plant exocyst subunit Sec5 to suppress

29 Aug $2017 \quad 1.0$

immunity, Summer School Environmental Signalling in Plants, Utrecht, The Netherlands

\section{- IAB interview}

\section{- Excursions}

Company visit to In2Care and Genetwister organized by EPS PhD

19 Sep 2014

0.2

council, Wageningen, The Netherlands

15 Apr $2014 \quad 1.0$

29 Nov $2015 \quad 1.0$

Company visit to Enza Zaden organized by EPS PhD council,

12 Jun 2015

0.2

Enkhuizen, The Netherlands

14 Oct 2016

0.2

Company visit to Tomato World organized by EPS PhD council, Honselersdijk, The Netherlands 


\section{3) In-Depth Studies}

date

$c p$

- Advanced scientific courses \& workshops

Spring School Host-Microbe Interactomics, Wageningen, The

Netherlands

Summer School Environmental Signaling in Plants, Utrecht, The

Netherlands

- Journal club

Thursday morning literature discussion in P. infestans group of

2014-2017 Phytopathology

Individual research training

\section{4) Personal Development}

$\underline{\text { date }}$

$\underline{c p}$

\section{- General skill training courses}

Competence Assessment

9 Sep $2014 \quad 0.3$

WSG PhD Workshop Carousel

17 Apr $2015 \quad 0.3$

Teaching and Supervising Thesis students

22-23 Sep 20150.6

Orientation on teaching for $\mathrm{PhD}$ candidates

15-17 Nov 20161.0

Lecturing

$3,10,17$ Feb $\quad 1.0$

2017

Exploring teaching outside academia: orientation and reflection

Sep - Nov $2017 \quad 4.0$

Teachers day WUR

12 Dec $2017 \quad 0.3$

- Organisation of meetings, $\mathrm{PhD}$ courses or outreach activities

Organisation of Get2Gether 2015

29-30 Jan $2015 \quad 1.5$

- Membership of EPS PhD Council

Member of EPS PhD council

Jul 2014 - Dec 1.0

2015

Subtotal Personal Development

10.0

TOTAL NUMBER OF CREDIT POINTS*

Herewith the Graduate School declares that the PhD candidate has complied with the educational requirements set by the Educational Committee of EPS with a minimum total of 30 ECTS credits.

*A credit represents a normative study load of 28 hours of study. 
This research was performed at the Laboratory of Phytopathology and the Laboratory of Cell Biology, Wageningen University \& Research, The Netherlands and financially supported by the Dutch Research Council (NWO) in the framework of the program Graduate School 'Uitgangsmaterialen', project number 831.13.003.

Cover design: Elysa Overdijk \& lliana Boshoven Gkini | AgileColor.com Layout design: Iliana Boshoven Gkini | AgileColor.com

Printed by: GVO drukkers \& vormgevers B.V. | gvo.nl 
